\title{
Thermoelectric Conversion of Waste Heat to Electricity in an IC Engine Powered Vehicle
}

\author{
Final Report \\ DEFC2604NT42281
}

Submitted to:

US Department of Energy

\author{
Prepared by: \\ Michigan State University \\ Iowa State University \\ Northwestern University \\ NASA Jet Propulsion Laboratory \\ Cummins Engine Company
}

April 30, 2011 


\section{DISCLAIMER}

This report was prepared as an account of work sponsored by an agency of the United States Government. Neither the United States Government nor any agency thereof, nor any of their employees, makes any warranty, express or implied, or assumes any legal liability or responsibility for the accuracy, completeness, or usefulness of any information, apparatus, product, or process disclosed, or represents that its use would not infringe privately owned rights. Reference herein to any specific commercial product, process, or service by trade name, trademark, manufacturer, or otherwise does not necessarily constitute or imply its endorsement, recommendation, or favoring by the United States Government or any agency thereof. The views and opinions of authors expressed herein do not necessarily state or reflect those of the United States Government or any agency thereof. 


\section{Project Participants}

\section{Michigan State University:}

Harold Schock, Professor, Mechanical Engineering, Principal Investigator Eldon Case, Professor, Chemical Engineering and Materials Science Jonathan D’Angelo, Graduate Student, Electrical and Computer Engineering Adam Downey, Graduate Student, Electrical and Computer Engineering Muhammad Farhan, Graduate Student, Electrical and Computer Engineering Tim Hogan, Associate Professor, Electrical and Computer Engineering Jason Johnson, Undergraduate Student, Chemical Engineering and Materials Science Kristen Khabir, Undergraduate Student, Chemical Engineering and Materials Science Muhammad Khan, Graduate Student, Electrical and Computer Engineering Daniel Kleinow, Undergraduate Student, Chemical Engineering and Materials Science Nuraddin Matchanov, Research Associate, Electrical and Computer Engineering Kevin Moran, Research Associate, Mechanical Engineering Jennifer Ni, Graduate Student, Chemical Engineering and Materials Science Fang Peng, Associate Professor, Electrical and Computer Engineering Adam L. Pilchak, Undergraduate Student, Chemical Engineering and Materials Science Trevor Ruckle, Research Associate, Mechanical Engineering Jeff Sakamoto, Assistant Professor, Chemical Engineering and Materials Science Robert Schmidt, Graduate Student, Chemical Engineering and Materials Science Stacey Schroeder, Undergraduate Student, Chemical Engineering and Materials Science Jarrod Short, Graduate Student, Electrical and Computer Engineering Ryan Stewart, Undergraduate Student, Chemical Engineering and Materials Science Edward Timm, Research Associate, Mechanical Engineering Bradley Wing, Undergraduate Student, Chemical Engineering and Materials Science Jim Winkelman, Research Associate, Mechanical Engineering ChuN-I Wu, Research Associate, Electrical and Computer Engineering Long Zhang, Research Associate, Chemical Engineering and Materials Science

Iowa State University:

Tom Shih, Professor and Chair, Department of Aerospace Engineering

Northwestern University:

Mercouri Kanatzidis, Professor, Chemistry

NASA Jet Propulsion Laboratory:

Thierry Caillat, Senior Member of Technical Staff

Jean-Pierre Fleurial, Technical Staff

\section{Cummins Engine Company:}

Wayne Eckerle, Executive Engineer, Research and Technology

Todd Sheridan, Technical Advisor, Advanced Engineering

Christopher Nelson, Technical Specialist 


\section{Table of Contents}

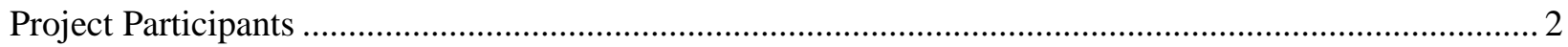

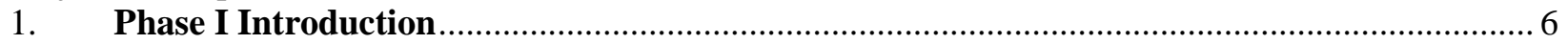

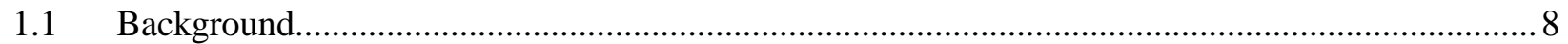

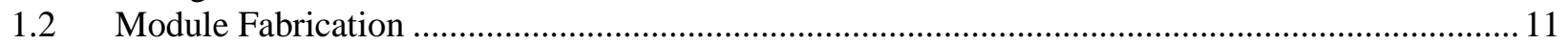

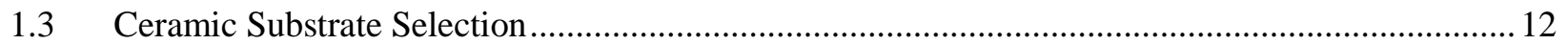

1.4 Sublimation and Oxidation Suppression and Thermal Insulation Integration.................................12

2. Wave Simulation Studies of TEG Applications to the Cummins 15 Liter ISX Diesel Engine........ 13

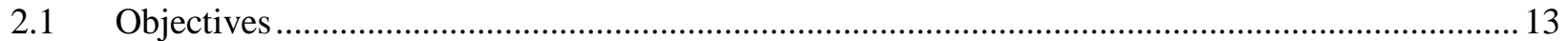

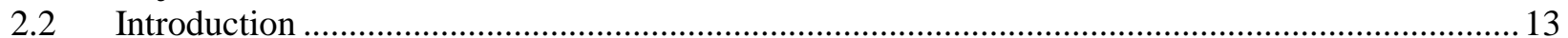

2.2.1 Description of the Cummins ISX Engine ................................................................................... 13

2.2.2 Description of the Relevant Features of the Ricardo WAVE Computer Model ............................... 15

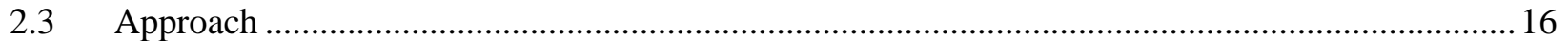

2.3.1 Single TEG Unit Per Engine Cylinder ................................................................................ 18

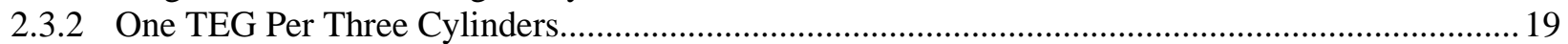

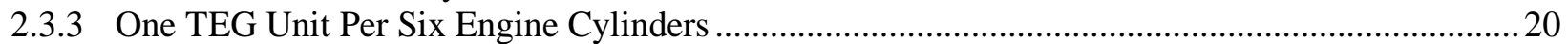

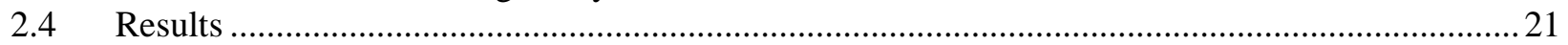

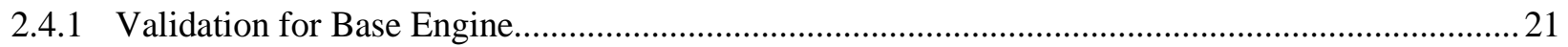

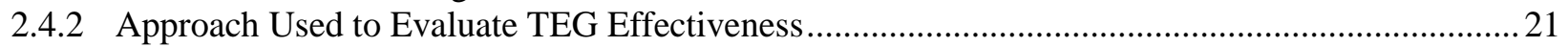

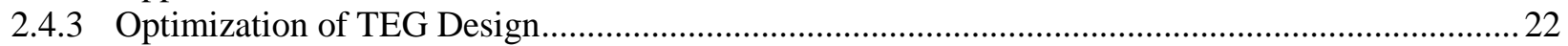

2.4.4 Effects of Unsteady Flow Dynamics on TEG Heat Transfer …………………………………........22

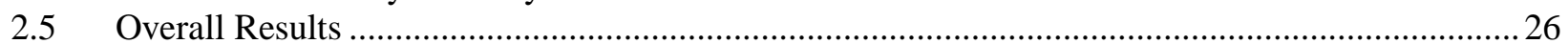

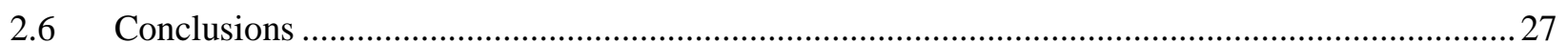

3. Heat Transfer Enhancements for Thermal Electric Power Generation …………………………... 27

3.1 Heat Transfer Enhancement for TEPG.................................................................................... 27

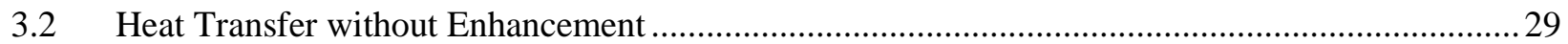

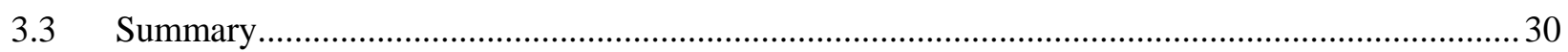

4. Selection of Thermoelectric Materials and Generator Design ........................................................ 30

4.1 High Performance TE P-Type Materials by Modification of $\mathrm{AgPb}_{18} \mathrm{SbTe}_{20}$ Materials (LASTT) .. 31

5. Electrical Energy Utilization ............................................................................................. 32

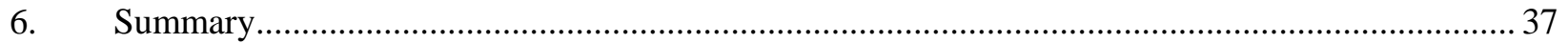

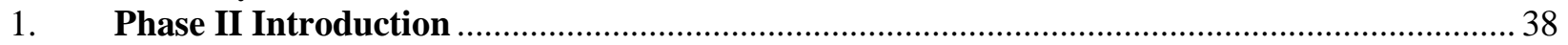

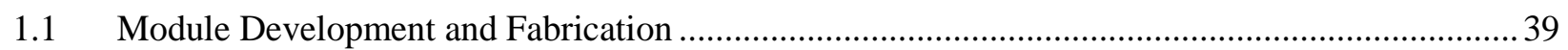

1.1.1 Skutterudite Leg Fabrication ............................................................................................. 39

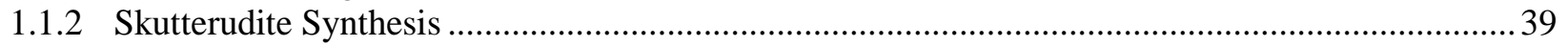

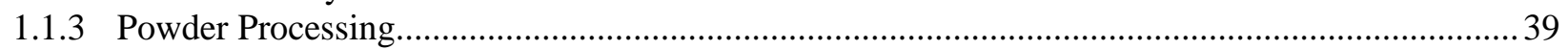

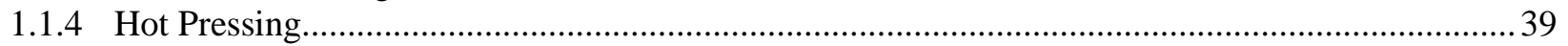

1.1.5 Unicouple Fabrication ...................................................................................................... 40

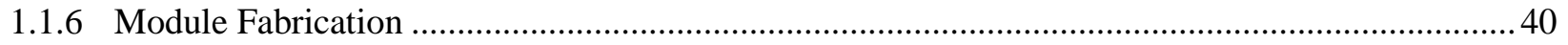

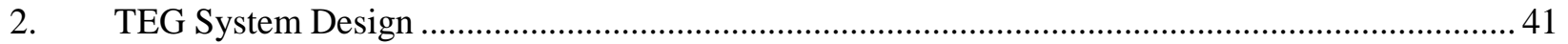

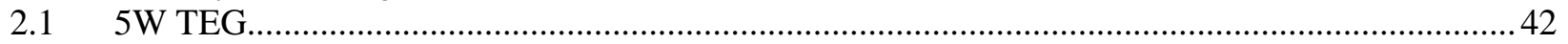

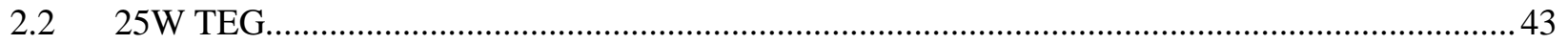

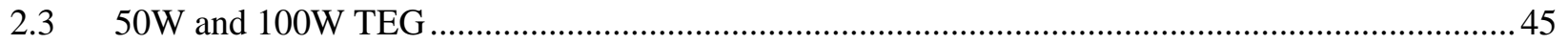

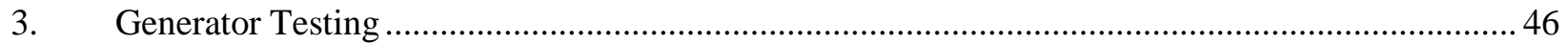

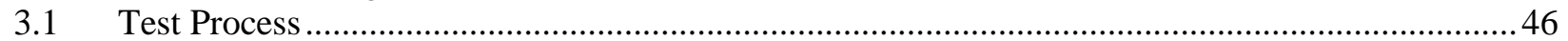

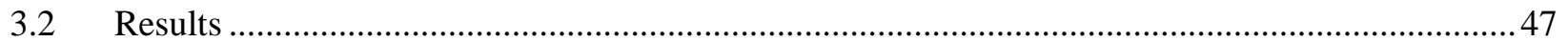

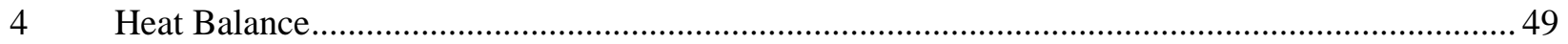

4.1 TEG System Temperatures.............................................................................................. 51

4.2 TEG Exit Temperatures............................................................................................

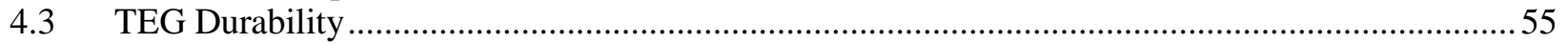




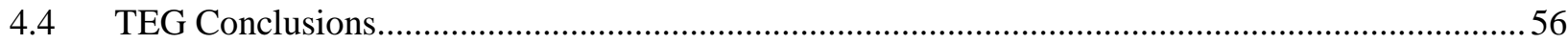

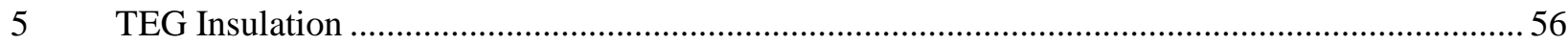

$5.1 \quad$ Aerogel Activities.................................................................................................................. 56

$5.2 \quad$ Aerogel Thermal Insulation Efficacy ………………………………………………………....5 5

$5.3 \quad$ Developing the Next Generation Aerogel Insulation ………………………………………….....5

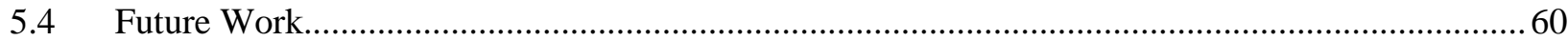

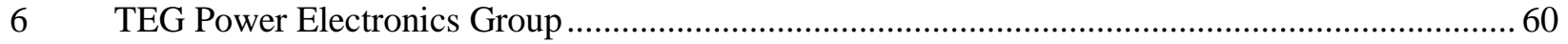

$6.11 \mathrm{Kw}$ 12x TEG Voltage Boost Circuit Prototype ……………………………………………......6

6.2 Four-Phase Interleaved ZCS-MMSCC for 5 kW Prototype..............................................................6 60

6.3 Conclusion and Plans for the Future........................................................................................... 63

7 Cost and Price Model of 1kW and 5kW Thermoelectric Based Auxiliary Power Units - Class 8

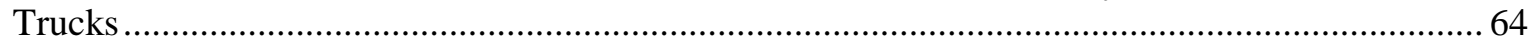

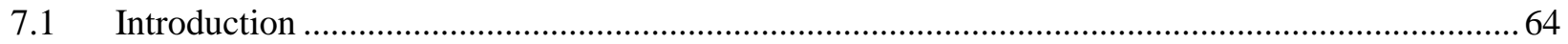

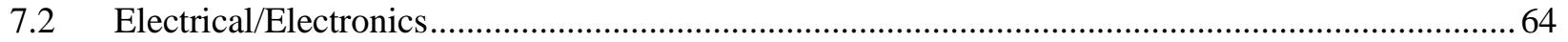

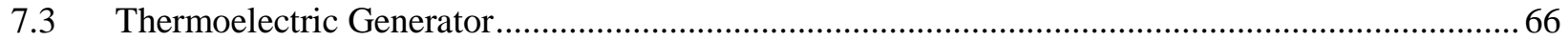

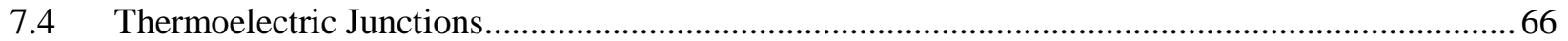

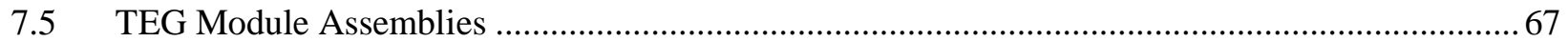

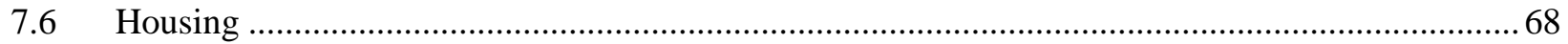

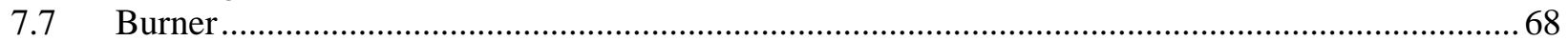

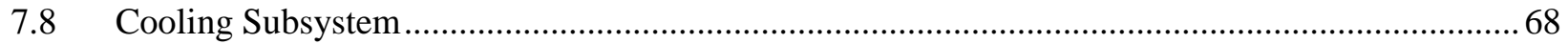

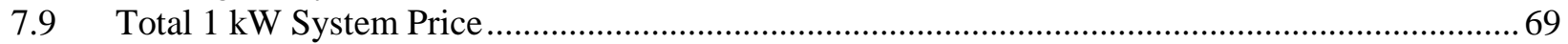

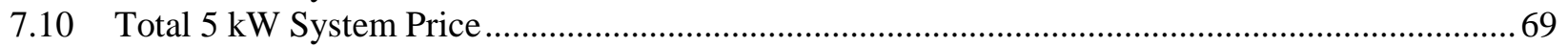

8 Material Research, Mechanical Properties and Thermoelectric Property Measurements ................ 70

8.1 Material Research - LAST and LASTT Cast Materials ................................................................... 70

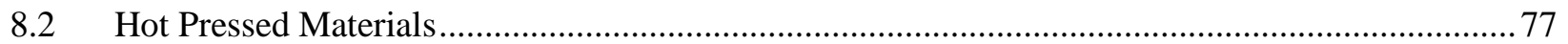

8.3 Module Fabrication with LAST and LAST Materials.................................................................. 83

$9 \quad$ Material Research - Investigation of PbTe - PbS Systems ……………………………………..... 93

9.1 Scale Up Studies and Reproducibility of PbTe - PbS Thermoelectric Materials ............................. 93

$9.2 \quad$ Structural Characterization (20\% DOE, 80\% ONR) ................................................................. 94

9.3 Powder X-Ray Diffraction (PXRD) (20\% DOE, 80\% ONR) ………………………………….... 94

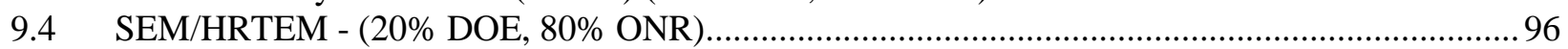

9.5 Thermoelectric Properties .................................................................................................. 98

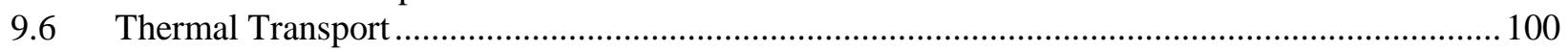

10 Powder Processing and Mechanical Properties of Skutterudite …………………………............... 102

10.1 Research on LAST (Lead-antimony-silver-tellurium) and LASTT (Lead-antimony-silvertellurium-tin) Thermoelectric Materials .........................................................................................103

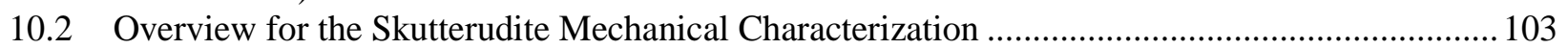

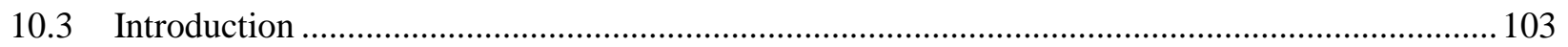

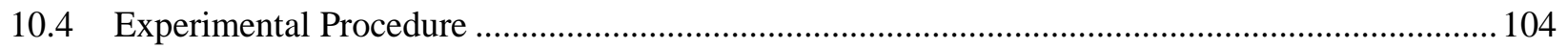

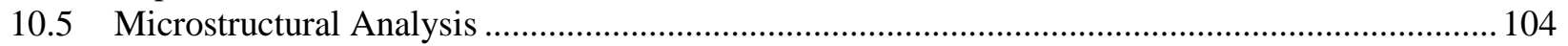

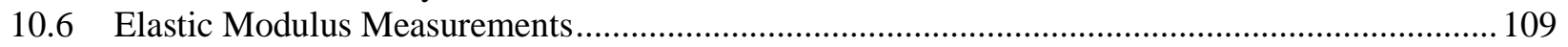

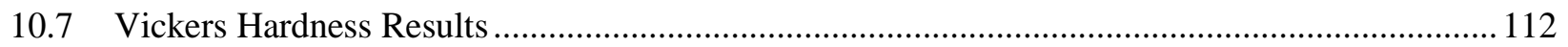

10.8 Thermal Expansion Results ................................................................................................. 112

10.9 Future Mechanical Characterization Work...………………………………………………........ 116

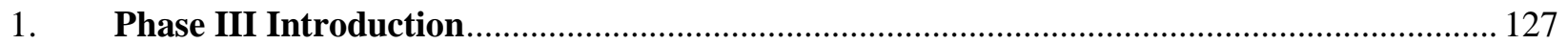

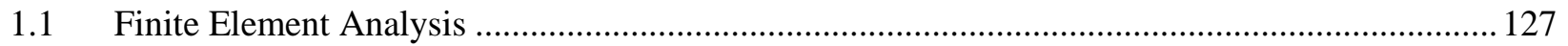

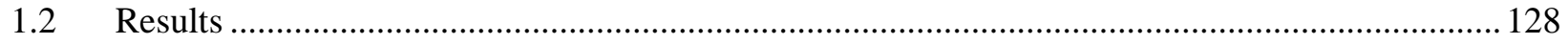

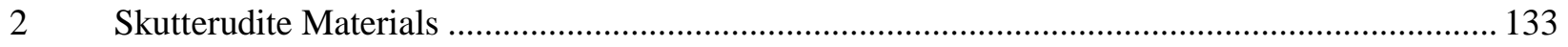

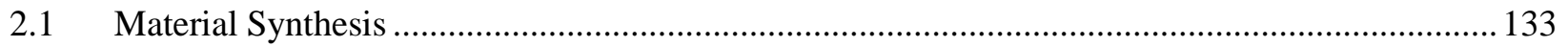

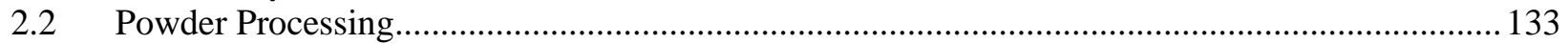

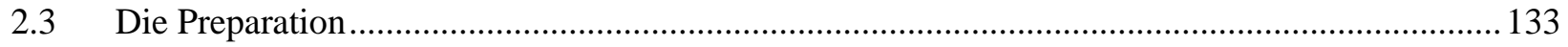




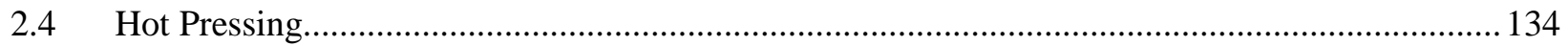

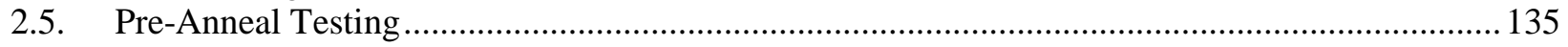

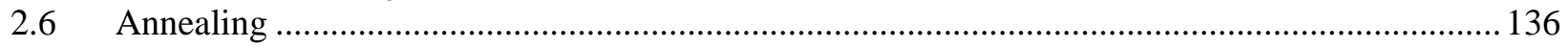

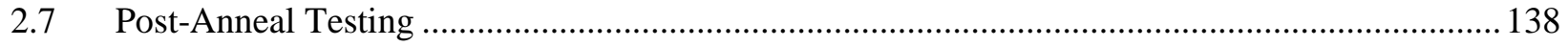

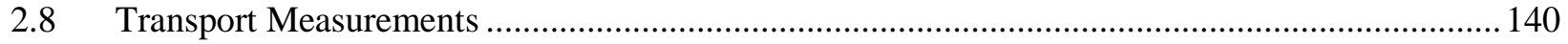

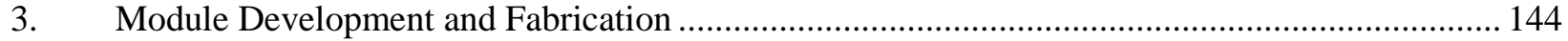

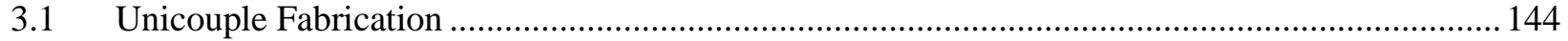

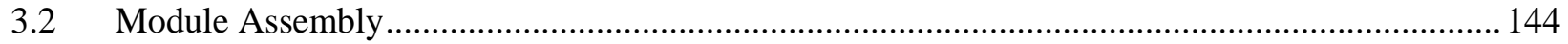

3.3 Changes from Previous Fabrication Process …......................................................................... 145

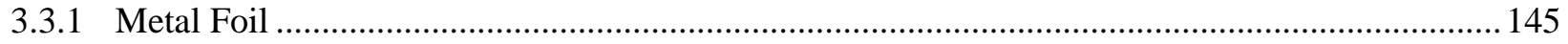

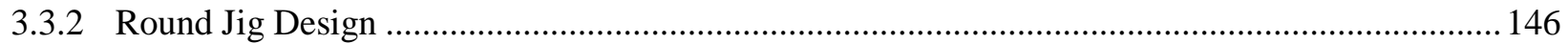

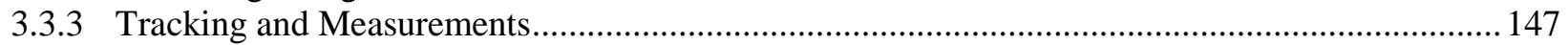

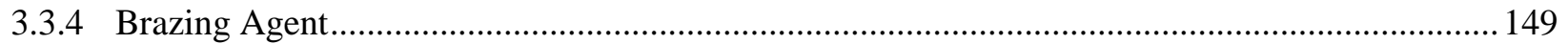

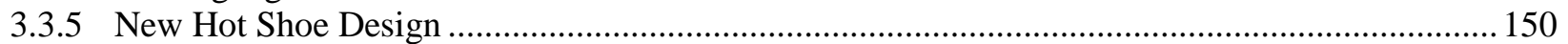

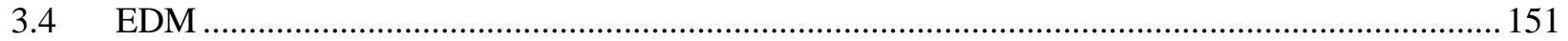

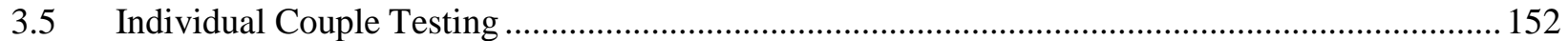

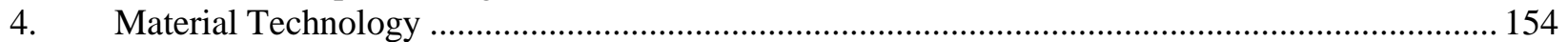

4.1 Functionally Graded Material Methodology in Thermoelectric Module Development................154

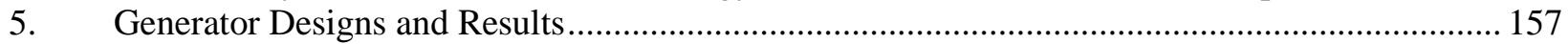

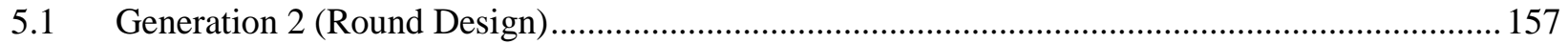

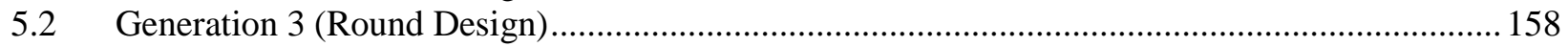

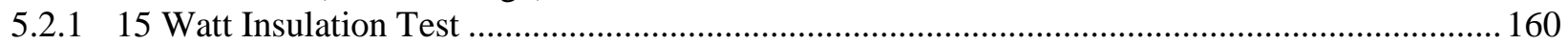

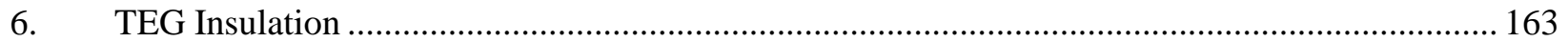

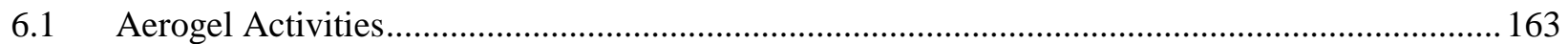

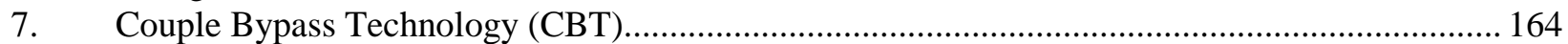

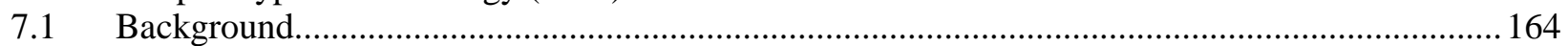

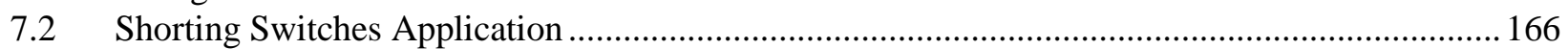

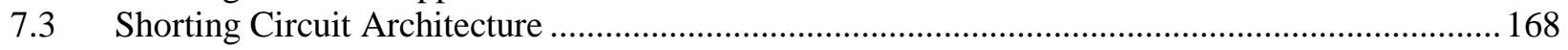

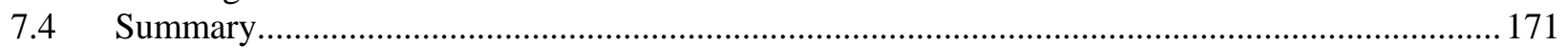

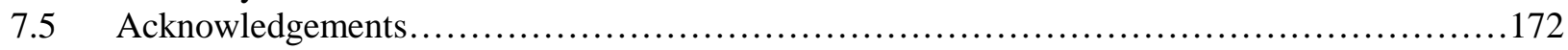

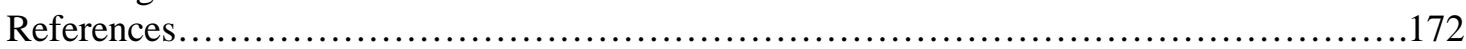

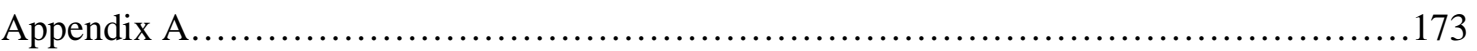

Appendix B......................................................................... 176 


\section{Phase I \\ 1. Introduction}

The Phase I work provided an evaluation of the use of new thermoelectric materials implemented into a direct energy conversion device to extract electrical energy from the exhaust gases of an over the road Class 8 diesel powerplant. The best current internal combustion engines have a nominal brake efficiency of $40 \%$, with $35 \%$ of the fuel energy going to exhaust, and $25 \%$ to other losses such as engine. Thus, in the best IC engines $60 \%$ of the energy content in the fuel is rejected as heat. The Phase I effort described the technology barriers to overcome for successful implementation of thermoelectric technology to the application described.

Although the potential exists for this substantial energy recovery at full power engine output, realistic duty cycles must be examined to critically evaluate potential energy recovery. Such cycles include the mode of operation, electrification of ancillaries, and potential hybridization. In the Phase I effort we considered a relatively conservative operating condition and conducted a detailed analysis of the potential benefits of implementation of this technology for the Class 8 truck application. A significant issue that must be resolved, if thermoelectric devices of practical utility are to be implemented in powertrain systems, is the determination of the configuration of the heat exchanger-thermoelectric device that will offer sufficient energy recovery to justify the cost. Having established a representative operating condition, a detailed engine energy analysis was conducted to evaluate the temperature gradients and heat fluxes available for energy conversion using a thermoelectric generator (TEG). Cummins Engine Company has provided the major guidance in terms of details related to engine operating mode for the application examined. In the Phase I effort, the Michigan State University (MSU) team has established the viability of the power conditioning and energy conversion configuration needed to effectively utilize the electrical energy generated.

We have evaluated the materials recently developed at MSU. We also have considered current materials and module designs of one of our project partners, NASA's Jet Propulsion Laboratory (JPL). The MSU materials were evaluated in conjunction with engine simulations and TEG thermal analysis. Critical to a realistic evaluation of thermoelectric technology for this application is the calculation of the temperature gradients available in the exhaust stream while maintaining the performance of the engine.

Using a different set of materials based on the skutterudite family, JPL has built high efficiency thermoelectric generators with proven reliability and efficiency. The JPL experience provides us an opportunity to leverage our effort with over 50 years worth of experience in the design, construction and cost analysis related to TEGs.

We believe that hot pressing will likely produce the highest quality thermoelectric material; part of the effort proposed was an evaluation of techniques that will assist in identifying low cost manufacturing alternatives. Tellurex Corp is one the leading US manufactures of thermoelectric devices and is a participant in this project. They have provided guidance in evaluation of materials and manufacturing methods for the materials identified as being most promising for this application. 
Currently, thermoelectric devices are commonly used in a variety of cooling and power generation applications. These devices include heat pumps. When electrical current is supplied to the device, a temperature gradient is established; when a temperature gradient is supplied across the device, electrical current will flow (if a load resistance is attached). Traditional uses include cooling electronics such as infrared detectors, laser diodes for fiber optics, CCD arrays and power generation applications such as radioisotope thermal generators (RTGs) like those used on Apollo's 12, 14, 15, and 17, Voyager's I and II, Galileo, etc. [1]

There are a number of advantages of thermoelectric devices over competing technologies including:

- high reliability (>250,000 hrs)

- silent and no vibration

- small electromagnetic signature

- temperature control to fractions of a degree

- not position dependent

- function in environments too severe, or too sensitive to conventional refrigeration

- small and lightweight

- no chlorofluorocarbons, chemicals, or compressed gases (nothing to replenish)

- environmentally "green"

- direction of heat pumping is fully reversible

The most significant disadvantage of thermoelectrics is the relatively low efficiency.

Presently, the best-known material for near-room temperature applications is based on $\mathrm{Bi}_{2} \mathrm{Te}_{3}$, as first reported in 1954 [2]. Making improvements beyond these materials is a formidable task. New fabrication capabilities and theoretical predictions [3] have helped to renew interest in this area of research and have led to a number of novel materials that show very promising thermoelectric properties. Some of these new materials along with the traditional materials are represented in Figure 1.1. ZT is a normalized figure of merit used to describe the performance of a thermoelectric material. For practical purposes, a ZT of a couple or segmented couple must be greater than one for the temperature range under consideration. 


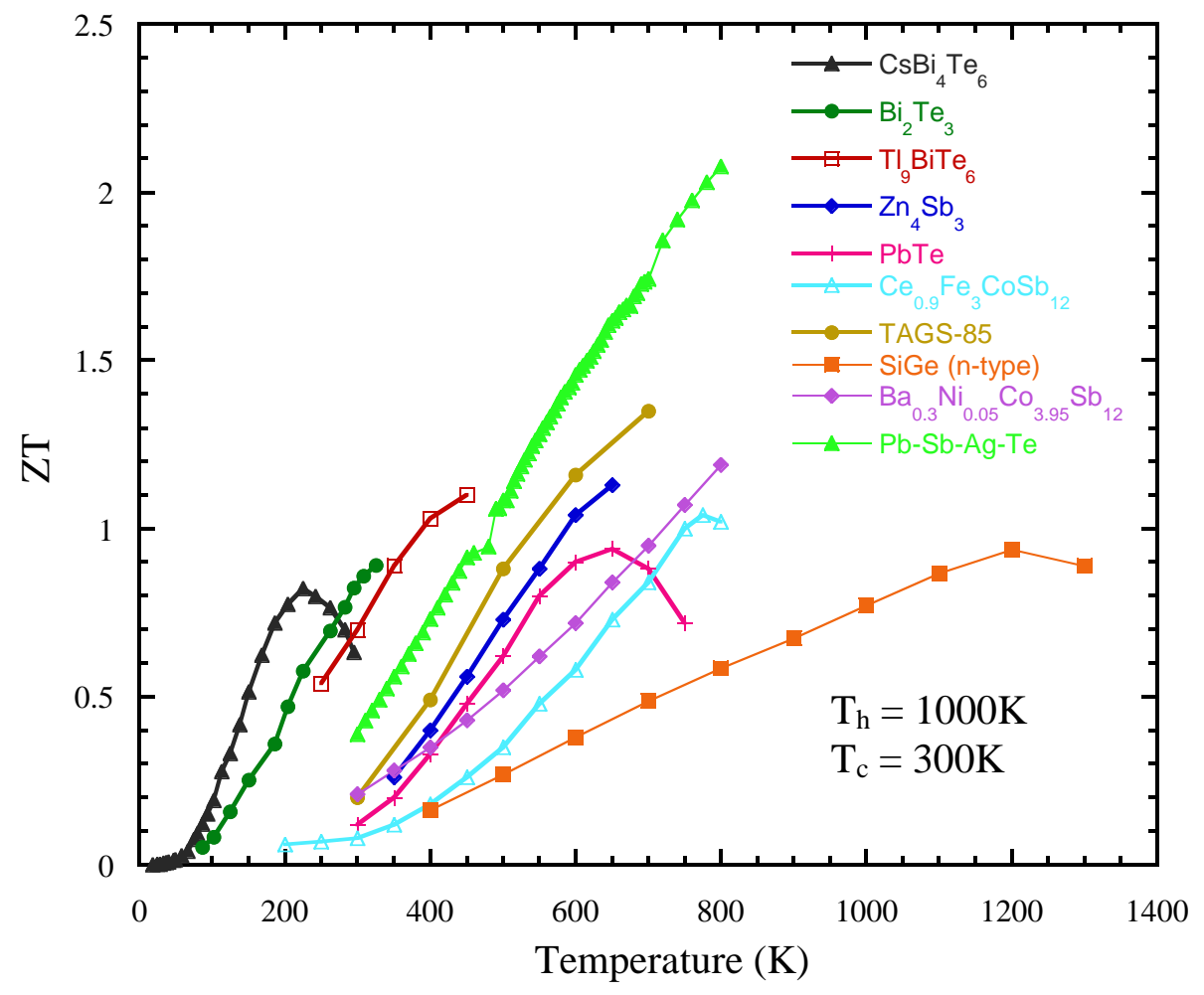

Figure 1.1. Temperature dependence of high ZT materials.

\subsection{Background}

New high performance thermoelectric materials (from 975 to 450K) were recently developed at JPL under the sponsorship of Office of Naval Research (ONR) and Defense Advanced Research Projects Agency (DARPA). These materials include $\mathrm{Zn}_{4} \mathrm{Sb}_{3}$, unfilled $\mathrm{CoSb}_{3}$ and filled skutterudite compounds based on $\mathrm{CeFe}_{4} \mathrm{Sb}_{12}$. Other laboratories, including Oak Ridge National Laboratory and Yamaguchi University (Japan), have independently confirmed these values.

Segmented couples incorporating a combination of state-of-the-art $\mathrm{Bi}_{2} \mathrm{Te}_{3}$-based thermoelectric materials and novel P-type $\mathrm{Zn}_{4} \mathrm{Sb}_{3}$, P-type $\mathrm{Ce}_{0.85} \mathrm{Fe}_{3.5} \mathrm{Co}_{0.5} \mathrm{Sb}_{12}$ and $\mathrm{N}$-type $\mathrm{CoSb}_{3}$-based skutterudite alloys have been proposed at JPL. The segmented couple (schematically illustrated in Figure 1.2) has the potential to achieve a thermoelectric efficiency of $\sim 15 \%$ over a $975 \mathrm{~K}$ $300 \mathrm{~K}$ temperature gradient. Preliminary temperature stability tests indicate that the maximum operating temperature is approximately $975 \mathrm{~K}$ for the skutterudites and approximately $675 \mathrm{~K}$ for $\mathrm{Zn}_{4} \mathrm{Sb}_{3}$. Above this temperature, $\mathrm{Zn}_{4} \mathrm{Sb}_{3}$ converts into a different crystallographic structure with less efficient thermoelectric properties. Because the new MSU materials have been reported to have a higher figure of merit within the temperature range of 800-400K (ZT 2 @800K) we have evaluated the possibility of combining the MSU materials with the skutterudites in a segmented configuration in order to achieve the highest possible efficiency. 


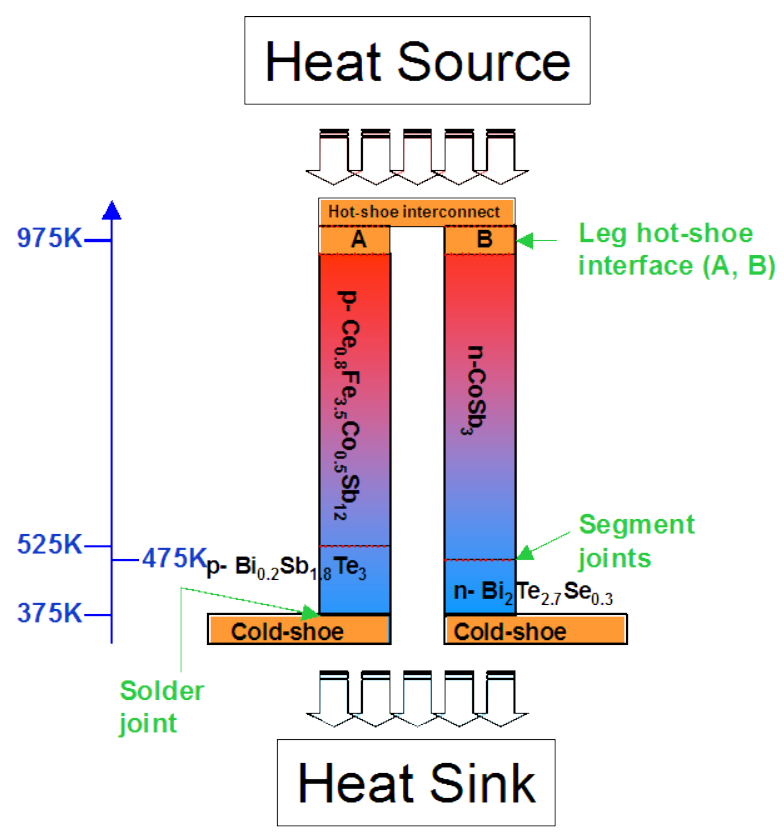

Figure 1.2. Advanced, segmented couple incorporating new high performance thermoelectric materials. The relative lengths of each segment and the cross-sectional areas for the $\mathrm{P}$ and $\mathrm{N}$-legs are drawn to scale.

In the optimal geometry for the segmented couple depicted in Figure 1.2, each segment has the same current and heat flow rate as the other segments in the same leg. Thus, in order to maintain the desired temperature profile (i.e., keeping the interface temperatures at their desired level), the geometry of the legs must be optimized. The segmented device configuration is achieved by considering primarily the differences in thermal conductivity, to achieve the desired temperature gradient across each material. Semi-empirical models have been developed at JPL to optimize the geometry of the legs and the efficiency of the couple.

Figure 1.3 shows the calculated thermal to electrical efficiencies for different state-of-the-art systems including PbTe, SiGe, $\mathrm{Bi}_{2} \mathrm{Te}_{3}$ and MSU AgPbSbTe-based systems. The advanced couples have the potential for nearly doubling the thermal to electrical efficiencies over state-ofthe-art systems.

The development of the advanced couples has been initiated at JPL, MSU and in other locations. Segmented legs have been fabricated by JPL using a diffusion bonding/hot-pressing process (see Figure 1.4). Limited life tests have been performed to date. Several couples have also been fabricated using a combination of powder metallurgy and brazing techniques. In a transportation application, the thermoelectric generator will experience large transients and variable temperature gradients from idle to full power operation. Even greater transients will be induced during start-up of a cold engine and cool-down of a hot engine. This will present significant challenges on the mechanical and metallurgical properties of these devices. 


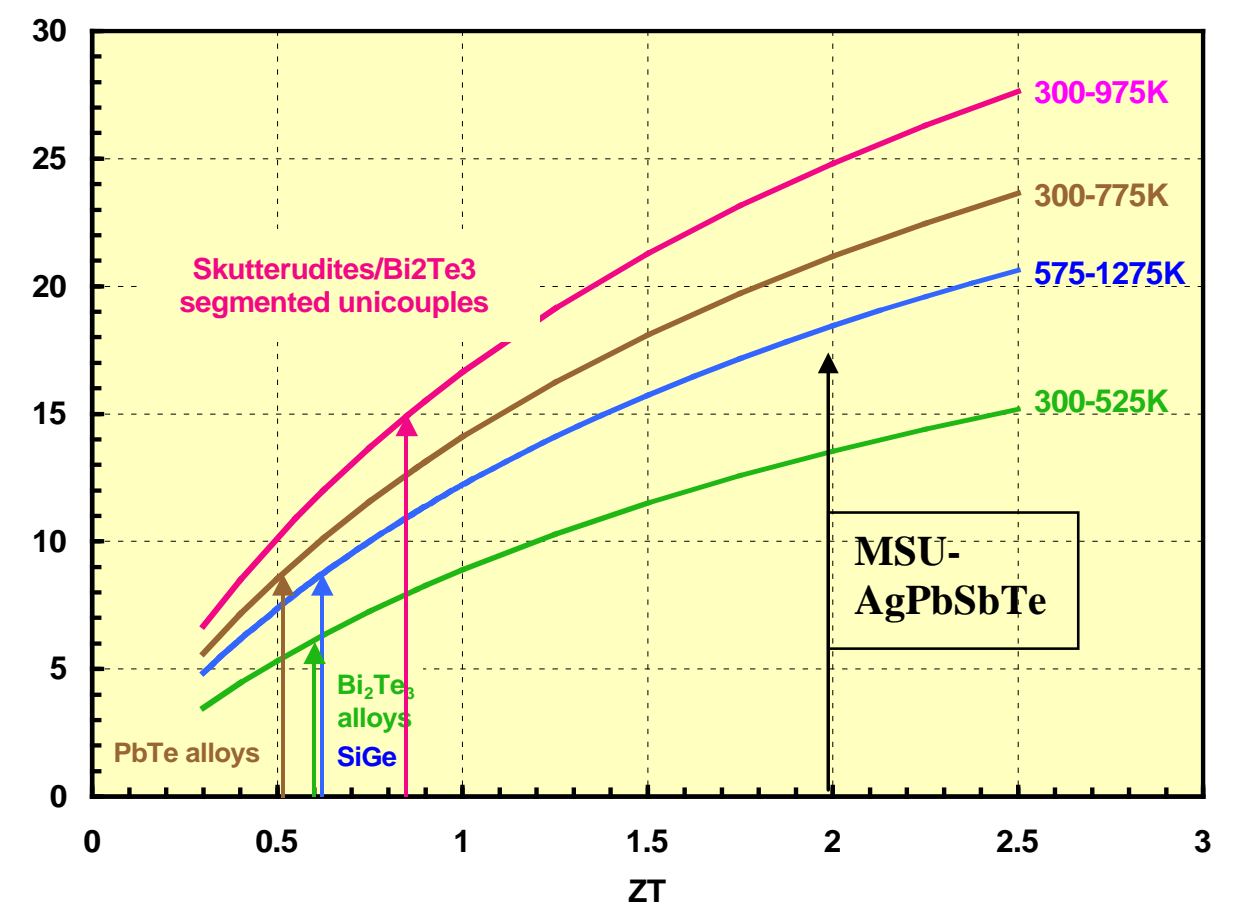

Figure 1.3. Calculated thermal to electrical efficiencies for advanced skutterudite-based couples compared to PbTe, SiGe, $\mathrm{Bi}_{2} \mathrm{Te}_{3}$ - and AgPbSbTe-based materials.

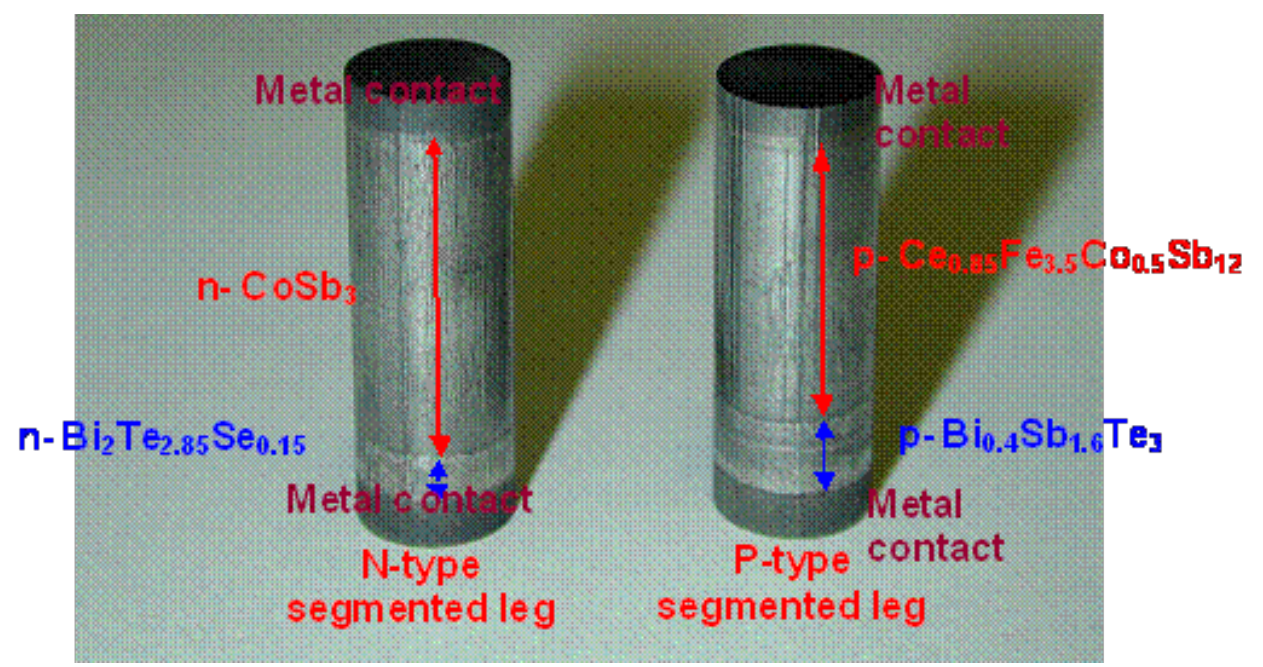

Figure 1.4. $\mathrm{Bi}_{2} \mathrm{Te}_{3} /$ skutterudite segmented legs fabricated by a diffusion bonding/hot-pressing process.

A $14 \%$ conversion efficiency has been experimentally demonstrated for $\mathrm{Bi}_{2} \mathrm{Te}_{3}$ skutterudite couples operating between $975 \mathrm{~K}$ and $300 \mathrm{~K}$. This fully validates the high measured ZT values and the predicted device performance (see Figure 1.5). This result has been independently confirmed at the University of New Mexico. Further development of these couples is currently supported by NASA to evaluate its potential and feasibility for integration into high performance and high specific power Radioisotope Power Systems. 


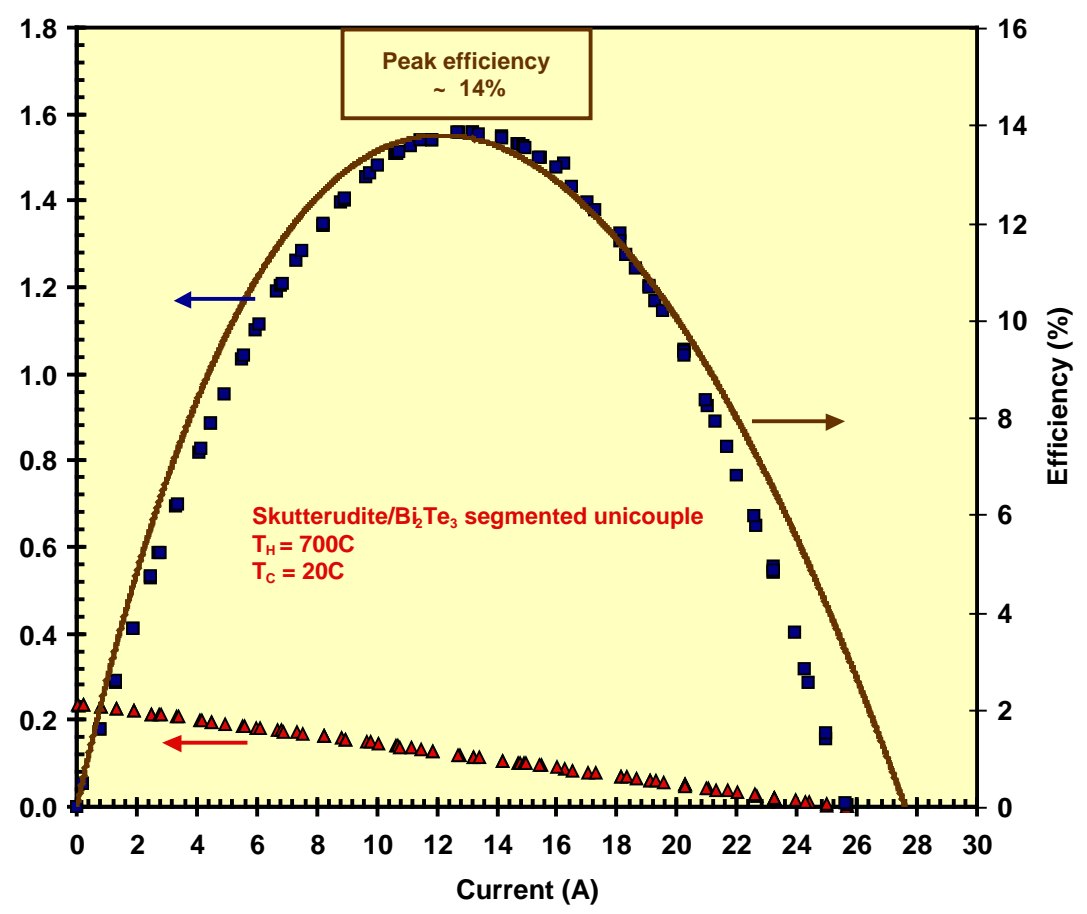

Figure 1.5. Experimental power and voltage output as a function of load current for a $\mathrm{Bi}_{2} \mathrm{Te}_{3} /$ skutterudite segmented couple operating at cold-side temperature of $300 \mathrm{~K}$ and a hot-side temperature of $975 \mathrm{~K}$.

The data shown in Figure 1.5 is in excellent agreement with the theoretically predicted values based on the thermoelectric properties of the materials. The inferred peak efficiency is nearly $14 \%$ at one-half of the open circuit voltage. Although this is the best known verified couple efficiency, the temperature range is considerably higher than expected for average exhaust of the diesel engine.

\subsection{Module Fabrication}

Thermoelectric modules are comprised of thermoelectric $\mathrm{N}$ and P-type legs connected electrically in series and thermally in parallel. Fabrication typically involves: 1) consolidating thermoelectric powder through standard powder metallurgical processes, 2) dicing the thermoelectric legs into the appropriate dimensions, 3) bonding thermoelectric legs to a premetallized, non-electrically conductive substrate either through soldering, brazing or diffusion bonding, and 4) integrating suitable thermal insulation.

At present, JPL has the capability to fabricate skutterudite-based couples capable of delivering over $14 \%$ conversion efficiency. All the appropriate technology required to achieve this will be directly integrated into the proposed module development with additional activities to include the development of advanced fabrication processes and materials selection for components such as ceramic substrates, sublimation suppression/oxidation coatings and thermal insulation.

Although interconnects have been made with brazing technology, at present the most promising interconnect technology developed at JPL involves diffusion bonding. Extremely low contact resistance has been achieved with this technique and life tests indicate that the bond is very 
stable at 700C for extended periods of time. Diffusion bonding is a process that involves solidstate diffusion between a metalized TE leg and the metal interconnect. Because this is a solidstate process, precise alignment between components and significant pressure must be applied to make the bond. This will require the development of advanced "egg crates" (a term used in industry to describe scaffolding to align thermoelectric legs with the metalized circuitry on the ceramic substrates). Currently, JPL is investigating advanced egg crates to include stiff vaporizable polymers, which will simplify fabrication.

\subsection{Ceramic Substrate Selection}

The purpose of the ceramic substrate is to electrically isolate the interconnect circuitry. Ideally, the ceramic substrate should have low electrical conductivity and high thermal conductivity, since it is arranged in series with respect to heat flux through the module. Additionally, to minimize thermal stresses the substrate should have a coefficient of thermal expansion that is compatible with the interconnects and heat exchanger surfaces. Examples of potential ceramic substrates are aluminum oxide (polycrystalline corundum or single crystal sapphire oriented in the a-plane) aluminum nitride and beryllium oxide. Once prospective ceramic substrates have been selected, methods for metalizing and patterning must be developed. For this, photolithography and electrochemical or vapor deposition will be considered along with standard printed circuit board patterning techniques. Tests will also be conducted to establish acceptable bond stability between the metal interconnects and the ceramic substrates.

\subsection{Sublimation and Oxidation Suppression and Thermal Insulation Integration}

Tellurium and antimony sublime readily from $\mathrm{Bi}_{2} \mathrm{Te}_{3}, \mathrm{PbTe}$, TAGS and $\mathrm{CoSb}_{3}$, which degrades the material integrity and device performance. JPL has recently developed coatings that can significantly suppress sublimation of antimony from skutterudite antimonides. To suppress sublimation, skutterudite legs can be encapsulated with thin, robust coatings. These coatings can consist of thin metal foils of material A or molybdenum. Although the films are thin enough to minimize thermal and electrical shorting (which can potentially diminish performance), coatings that are both electrically and thermally insulating are preferred. Recent work has identified what could be described as the ideal coating: aerogel. Aerogel is typically known as an extremely porous ( $>99 \%$ porous) silicon dioxide. It has very low thermal and electrical conductivity. Aerogel has interconnected pores, which are generally in the range of angstroms to a few nanometers. As such, the path required for metal vapor to permeate aerogel is extremely tortuous. Previously, it was determined that the mean free path of Sb vapor under predicted operating conditions (700C and $10^{-6}$ torr) is in the range of centimeters or perhaps longer. Thus, it is hypothesized that the presence of an aerogel barrier on the surface of skutterudites should impede Sb vapor transport and effectively act as a continuous surface. This is based on the fact that the mean free path of Sb vapor is far greater than the pore dimensions in the aerogel. When skutterudite coupons are not encapsulated, Sb depletion bands form on the outer surface and advance toward the center until only CoSb is left. However, when the same coupons are encapsulated in aerogel there almost no Sb loss or depletion bands that form. Another added benefit of using aerogel as a coating is that it can also serve as thermal insulation. Because it is so porous, aerogel has extremely low thermal conductivity. Also, since aerogel is made through liquid synthesis it can be cast in or around thermoelectric devices or modules, thus assisting in channeling heat through the thermoelectric legs and eliminating lateral heat loss. Altogether, aerogel coatings could significantly improve skutterudite thermoelectric device durability and performance by acting as an effective sublimation barrier as well as effective thermal insulation. 
For large diesel power plant vehicles, DOE is currently sponsoring conventional electrification work with Caterpillar, Inc. [4] to investigate use of electrified accessories for load reduction, electric turbo compounding as means for energy recovery from exhaust gases as well as additional electric truck efforts. The investigation of accessory load reduction is of particular interest to this TEG proposal because Class 8 truck electrical loads are now in the $4 \mathrm{~kW}$ range, increasing to $8 \mathrm{~kW}$. There are also efforts aimed at eliminating vehicle idle in OTR trucks (now $\sim 1830 \mathrm{~h}$ /year) through the use of auxiliary power units such as small 2-cylinder diesel gen-sets and fuel cell power supplies. Also, shore power is being supplied in some states where nonidling is state law. This study focuses on the implementation of TEGs into the truck diesel power plant to offset engine driven generator capacity.

\section{Wave Simulation Studies of TEG Applications to the Cummins 15 Liter ISX Diesel Engine}

\subsection{Objectives}

The overall objective of this work is to analytically quantify the potential benefits of alternate thermoelectric generator (TEG) designs in converting waste heat from internal combustion engines to useful electrical power. The Ricardo WAVE Engine System Performance Simulation is used to model the Cummins ISX, 15 liter, 6-cylinder, diesel engine both in current baseline configuration and with various TEG designs integrated into the exhaust system. The studies are carried out for engine operating conditions most representative of those for an on-road, eighteenwheeled, Class 8 truck. Based on a preliminary analysis of heat sources within the engine system, the TEG was located in the exhaust system immediately downstream of the of the engine's exhaust manifold, where it could serve a dual role of electric generator and replacement for the existing EGR (Exhaust Gas Recirculation) cooler used for emissions control.

The study included the optimization of the TEG design parameters to extract the most heat possible from the exhaust gases and prediction of the TEG wall material temperatures that result. These temperatures are critical to the electrical conversion efficiency of the TEG itself.

\subsection{Introduction}

\subsubsection{Description of the Cummins ISX Engine}

The Cummins ISX engine is a direct-injection, turbo-charged, inline 6-cylinder diesel engine with a $137 \mathrm{~mm}$ bore diameter and $169 \mathrm{~mm}$ stroke, thereby displacing 14.95 liters (912.2 cubic inches). It employs a variable geometry turbo-charger with intercooler, and an exhaust gas recirculation (EGR) cooler to meet federal emissions requirements. The primary application of the ISX engine, and the one of interest for this analysis, is in Class 8, on-road, 18-wheeled trucks. Other applications include stationary power generation, construction and agricultural equipment, and recreational vehicles. 


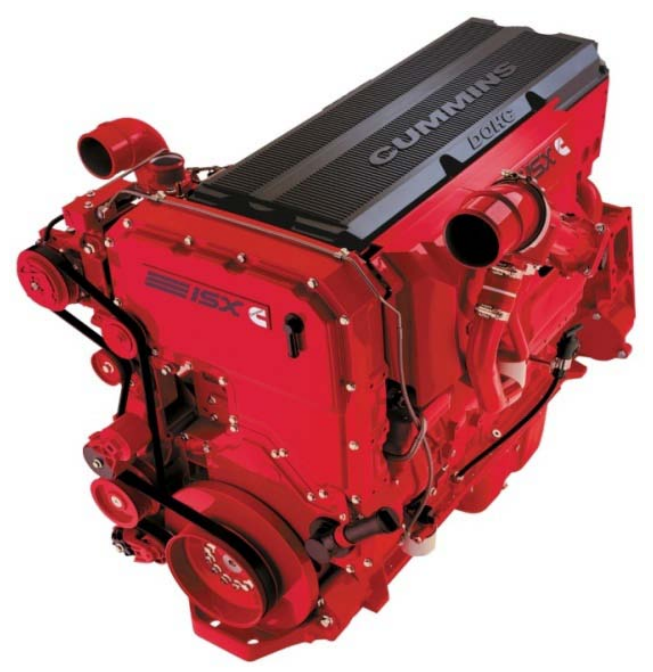

Figure 2.1. Cummins ISX 6-cylinder diesel engine

Table 2.1. ISX Engine operating conditions for ESC duty cycle model

\begin{tabular}{|c|c|c|c|c|c|c|}
\hline \multirow[t]{2}{*}{ Modes } & & A-25 & A-100 & B-62 & B-100 & C-100 \\
\hline & Units & & & & & \\
\hline Engine Crankshaft Speed & $\mathrm{rpm}$ & 1230.00 & 1230.00 & 1500.00 & 1500.00 & 1800.00 \\
\hline Torque & $\mathrm{ft}-\mathrm{lb}$ & 472.15 & 1886.80 & 1170.20 & 1887.30 & 1577.70 \\
\hline BMEP & psi & 78.05 & 311.92 & 193.45 & 312.00 & 260.82 \\
\hline \multirow[t]{2}{*}{ Power } & $\mathrm{HP}$ & 110.58 & 441.88 & 334.22 & 539.02 & 540.72 \\
\hline & $\mathrm{kW}$ & 82.46 & 329.52 & 249.23 & 401.96 & 403.22 \\
\hline \multirow[t]{2}{*}{ Fuel Rate } & $\mathrm{lb} / \mathrm{hr}$ & 40.30 & 147.76 & 111.88 & 183.56 & 196.64 \\
\hline & $\mathrm{kg} / \mathrm{hr}$ & 18.28 & 67.02 & 50.75 & 83.26 & 89.20 \\
\hline Air Flow Rate & $\mathrm{lb} / \mathrm{min}$ & 19.81 & 47.78 & 46.71 & 63.55 & 68.42 \\
\hline Fuel-Air Ratio & & 0.034 & 0.052 & 0.040 & 0.048 & 0.048 \\
\hline EGR Mass Flow & $\mathrm{lb} / \mathrm{min}$ & 7.18 & 9.87 & 12.80 & 15.96 & 13.22 \\
\hline EGR Fraction of Intake Charge & & 0.27 & 0.17 & 0.22 & 0.20 & 0.16 \\
\hline Barometer & $\mathrm{Hg}$ abs & 29.43 & 29.43 & 29.43 & 29.43 & 29.44 \\
\hline Intake Manifold Pressure & $\mathrm{Hg}$ abs & 13.90 & 61.21 & 46.19 & 73.57 & 62.38 \\
\hline PBOOST & bar & 1.47 & 3.07 & 2.56 & 3.49 & 3.11 \\
\hline Intake Manifold Temperature & ${ }^{\circ} \mathrm{F}$ & 120.07 & 121.78 & 114.42 & 130.47 & 135.12 \\
\hline TBOOST & ${ }^{\circ} \mathrm{K}$ & 322.08 & 323.03 & 318.94 & 327.86 & 330.44 \\
\hline Exhaust Manifold Pressure & $\mathrm{Hg}$ abs & 18.37 & 70.19 & 54.14 & 85.95 & 79.61 \\
\hline PBACK & bar & 1.62 & 3.38 & 2.83 & 3.91 & 3.70 \\
\hline Exhaust Manifold Temperature & ${ }^{\circ} \mathrm{F}$ & 733.27 & 1170.90 & 945.27 & 1159.60 & 1233.60 \\
\hline TBACK & ${ }^{\circ} \mathrm{K}$ & 662.74 & 905.87 & 780.52 & 899.59 & 940.71 \\
\hline
\end{tabular}

Based on information provided by Cummins, the ISX is typically tested at five primary operating Speed/Load "modes" that approximate conditions encountered in the ESC (European SteadyState Cycle) duty cycle for Class 8 truck applications. Table 2.1 lists these 5 points and the corresponding experimentally determined values of several operating parameters of interest. 
In Table 2.1, the five points include three engine speeds of: (A) $1230 \mathrm{rpm}$, (B) $1500 \mathrm{rpm}$, and (C) $1800 \mathrm{rpm}$. Each speed includes a full (100\%) load point. Part load points are included for $1230 \mathrm{rpm}-25 \%$ of full load (A-25) and $1500 \mathrm{rpm}-62 \%$ of full load (B-62). The latter B-62 operating point is the most representative of a "cruising" mode for Class 8 trucks and based on the recommendation of Cummins Engine was the focus of most of the modeling results to follow.

\subsubsection{Description of the Relevant Features of the Ricardo WAVE Computer Model}

The WAVE engine performance simulation is a commercially available CAE tool that has been used in the development of internal combustion engines for over twenty years. This simulation tool primarily aids in the design of induction and exhaust system components to optimize wideopen throttle performance and inlet/exhaust pipe noise levels and sound quality. The core governing equations within the WAVE code simulate the unsteady, compressible gas flow throughout the entire breathing system of the engine from the air inlet to the intake valves and from the exhaust valves to the "tailpipe" exit. Although the partial differential equations governing the gas flow are mathematically one-dimensional, they are in the classical "Quasi-1D” form (with the dimensional axis aligned along the centerline axis of each duct), which allows the inclusion of such 3-dimensional effects as cross-sectional area variation and heat transfer to the duct walls at each duct cross-section. This feature has been the primary reason for the usefulness of such models to accurately predict the performance of piston engines within a reasonable computer processing time interval. Fully three-dimensional fluid dynamic simulation (CFD) codes are not currently practical for predicting the thermo-fluid dynamic behavior throughout the entire breathing system of IC engines. For these reasons, Quasi-1D codes are by far the dominant CAE tool used in industry to predict the effects of engine design changes on performance and fuel consumption.

WAVE includes a comprehensive array of key physical sub-models that predict the thermal and fluid mechanical state of the engine for the more important engine design and operating parameters. These parameters include engine speed, load, fuel injection timing and spray characteristics, fuel-air ratio, inlet gas and fuel conditions (pressure, temperature, and composition), turbo-charger design, and practically all relevant geometric dimensions of the engine components.

The sub-models most relevant to the current study include governing physical equations for the following:

- in-cylinder combustion and heat transfer to the coolant and oil via the piston, bore walls, and cylinder head; including prediction of wall temperatures and heat flux rates

- heat transfer from the gases within the intake and exhaust system ducting to the surrounding structures and, ultimately, to the coolant or ambient, again including wall temperatures and heat flux (both instantaneous and integrated over the engine operating cycle). There are also provisions for multi-layered duct walls of differing material properties, which are important to this study for modeling the complex layered design of the TEG.

- unsteady, compressible, fluid dynamic behavior of the same gases within the intake and exhaust ducting, including instantaneous and cycle-averaged gas temperature, total enthalpy, 
and wall heat flux. The gases consist of air, fuel vapor, and burned gases from the previous engine cycles (both internal to the cylinder and "external” EGR).

- intake and exhaust port effective areas as functions of valve lift and flow direction

Having undergone continual development over the last twenty years, the WAVE software includes very user friendly pre- and post-processors to enable relatively efficient handling of the extensive input and output information involved.

\subsection{Approach}

As noted above, consideration of the possible sources of "waste heat" from the Cummins ISX diesel engine led to the conclusion that the exhaust gases are the highest in energy and most easily accessible. Their use offers the further benefit of replacing the existing EGR cooler with the TEG, thus enabling effective NOx emissions control.

Figure 2.2 shows one proposed design of a "Single Unit TEG" that could be used in the exhaust system of the ISX engine.

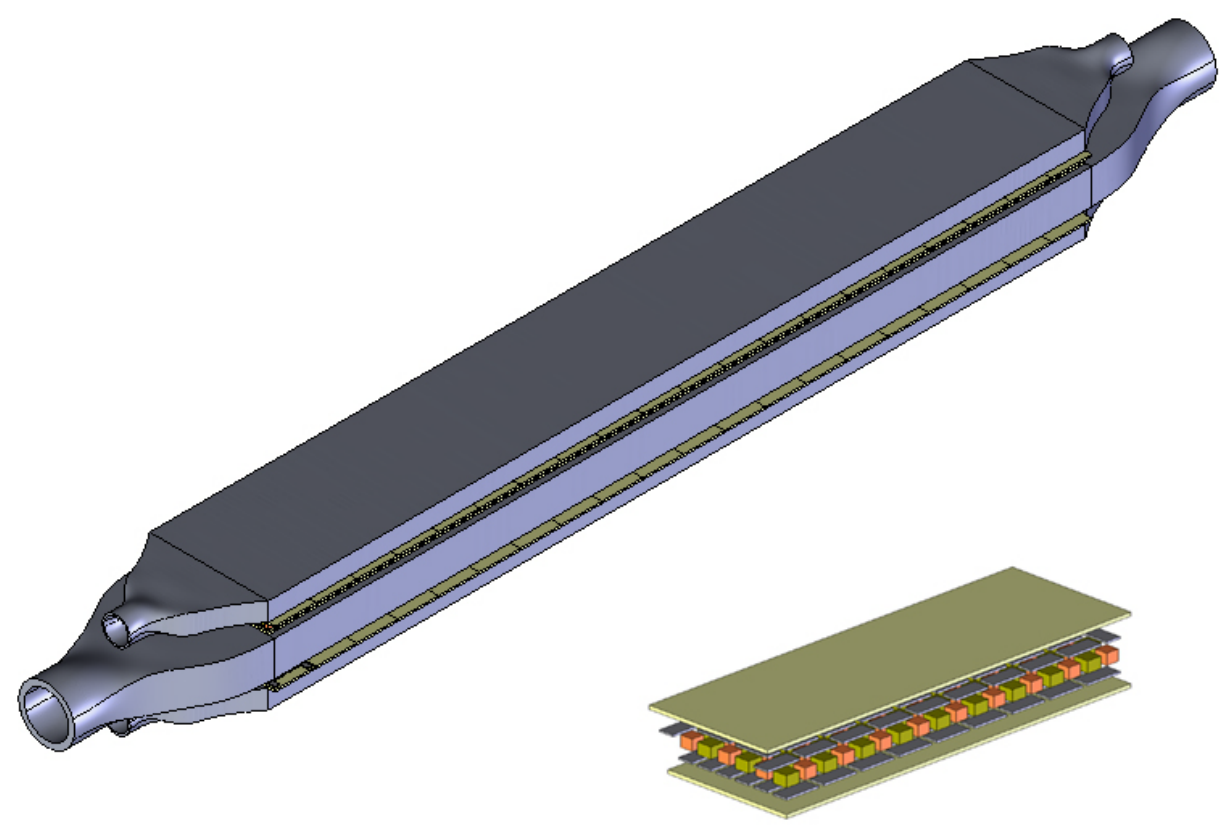

Figure 2.2. Single TEG with exploded view of a module.

This initial TEG design (culminating from CAD “packaging” studies) was intended to fit a sufficient number of P-N modules into a single TEG unit to produce approximately $1 \mathrm{~kW}$ of power, based on certain "best estimates" of P-N junction dimensions and efficiencies. The resulting design shown has $54 \mathrm{P}-\mathrm{N}$ junctions.

As illustrated in Figure 2.2, exhaust gases from the engine exhaust ports, or the exhaust manifold outlet (depending on the type of application, as discussed below), are indicated entering the left end of the TEG unit and exiting on the right end. Similar to a counter-flow heat exchanger, either liquid coolant or forced air enters on the right through separate ducting and 
exits on the left. Although the preferred TEG construction of Figure 2.2 is not easily modeled with the Quasi-1D approach, WAVE does allow one to account for most design features of interest, such as the non-circular duct cross-section shape and its attendant increased wall wetted surface area for heat transfer. Figure 2.3 illustrates how the TEG cross-section (perpendicular to the gas and coolant flow directions) would be represented with WAVE as a circular cross-section of the same "effective" flow and wetted wall areas.
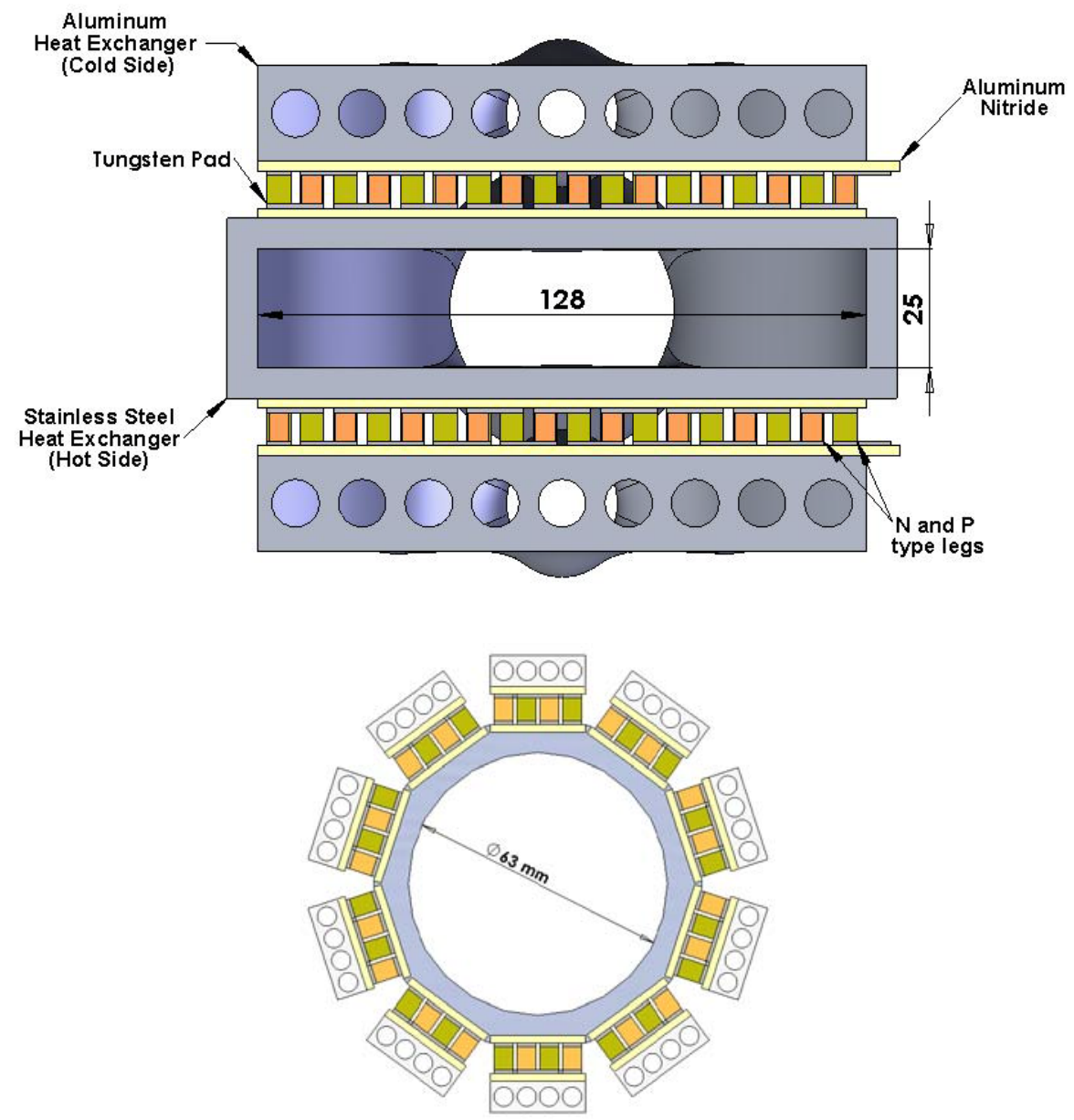

Figure 2.3. Cross section of rectangular and circular TEG.

Three basic options were considered in determining how best to configure these single unit TEG's in the exhaust system of the ISX engine. These options were chosen as they represent limits of exhaust gas to electrical energy conversion.

1. One single unit TEG per cylinder. This would require 6 units per engine and might be best for packaging the largest number of P-N modules per engine, hence generating the most electrical power. Figure 2.4 illustrates one such configuration.

2. One TEG unit per 3 cylinders (two TEG units per engine). This case seems to be a very reasonable alternative, because it would provide for three equally spaced (in time) exhaust gas "pulses" to each TEG unit per engine cycle (two engine crankshaft revolutions). This follows from a consideration of the firing order of the ISX engine and the fact that the 
interval between the opening events of each cylinder's exhaust valves is 120 crank angle degrees.

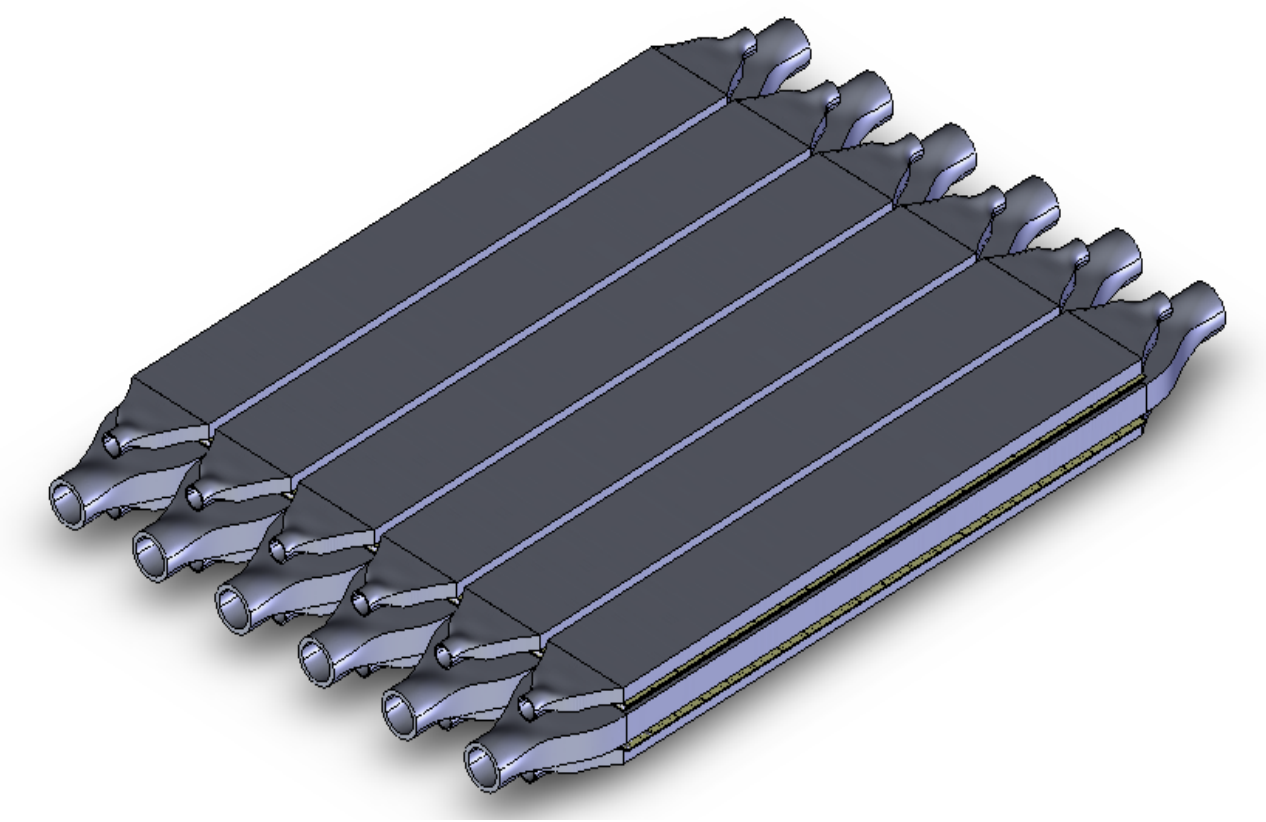

Figure 2.4. Six unit TEG

3. One TEG unit per six engine cylinders. This configuration would entail only one TEG unit for the entire engine and would provide the highest frequency of exhaust pulses to the TEG per engine cycle - three pulses per revolution, six per engine cycle. Although the heat loading to the TEG would be the highest of any configuration, the number of P-N modules exposed to this energy input would be the least due to packaging considerations.

\subsubsection{Single TEG Unit Per Engine Cylinder}

For this configuration (each cylinder exhausts into its own TEG), experiments have shown and WAVE modeling results confirm that with engine speeds below $2000 \mathrm{rpm}$, a six-cylinder engine behaves as six independent single cylinder engines. Therefore, the case of six TEG's (one per cylinder) was effectively modeled as a single-cylinder engine and as necessary, displacement specific results (e.g., torque and horsepower) multiplied by a factor of six. Figure 2.5 shows the simple single cylinder stick model used. This assumption decreases the computer processing times by over a factor of six and allows more extensive design optimization studies to be carried out. 


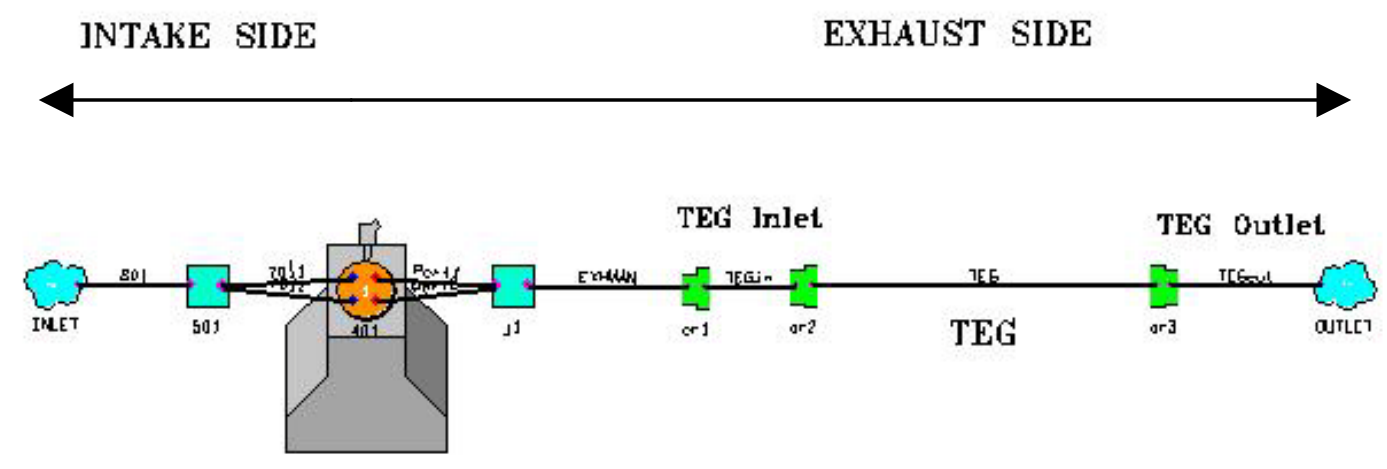

Figure 2.5. WAVE representation of single-cylinder engine with one TEG.

As seen in Figure 2.5, flow through the engine is from the left inlet ambient (shown as a blue "cloud") to the right exhaust ambient. Note that an ambient corresponds to an infinite volume plenum where thermodynamic properties such as pressure, temperature, and gas composition remain fixed at specified values during the entire simulation. These conditions can be those of the true ambient (i.e., atmospheric conditions), or they can represent other locations in the engine's intake and exhaust systems where conditions are approximately constant with time and are known to be from experimental data for the operating conditions of interest. The latter case can be useful to simplify the model and avoid having to calibrate and "carry along" computations for parts of the intake and/or exhaust systems that do not have a significant effect on the results of interest.

In this study, we make use of this approximation to avoid the complexities of modeling the variable geometry turbocharger of the Cummins ISX engine. Instead, we use the measured values of pressures and temperatures (1) at the outlet of the turbo-charger compressor and intercooler ("PBOOST" and "TBOOST" in Table 2.1); and (2) the inlet to the turbo-charger turbine ("PBACK" and "TBACK" in Table 2.1) to represent the inlet and exhaust ambient conditions, respectively.

Another important assumption used throughout this initial study of TEG behavior in IC engine exhaust systems is that all of the exhaust gas expelled from the cylinders passes through the TEG(s) before entering the exhaust ambient (turbine inlet). As a consequence, the results represent an ideal "upper bound" on the amount of energy that can be extracted by the TEGs. In the actual case of the ISX engine, over $50 \%$ of the exhaust gas will be bypassed through the TEG/EGR cooler and the rest will enter the turbine inlet. The turbine efficiency ultimately depends on the total enthalpy of its inlet gases. This delicate balance of energy distribution within the ISX exhaust system will depend strongly on the location and design of the TEG and of the turbo-charger/intercooler. These system energy balance issues will be addressed in detail in Phase II of this project.

\subsubsection{One TEG Per Three Cylinders}

Similar to the assumptions discussed above and confirmed by modeling studies, this configuration effectively behaves as two independent three-cylinder engines coupled mechanically, but independent in terms of the flow dynamics in the exhaust system. It can, 
therefore, be modeled as one 3-cylinder engine to facilitate shorter computer processing times and more practical design optimization studies. Figure $\mathbf{2 . 6}$ shows the model used in the analysis

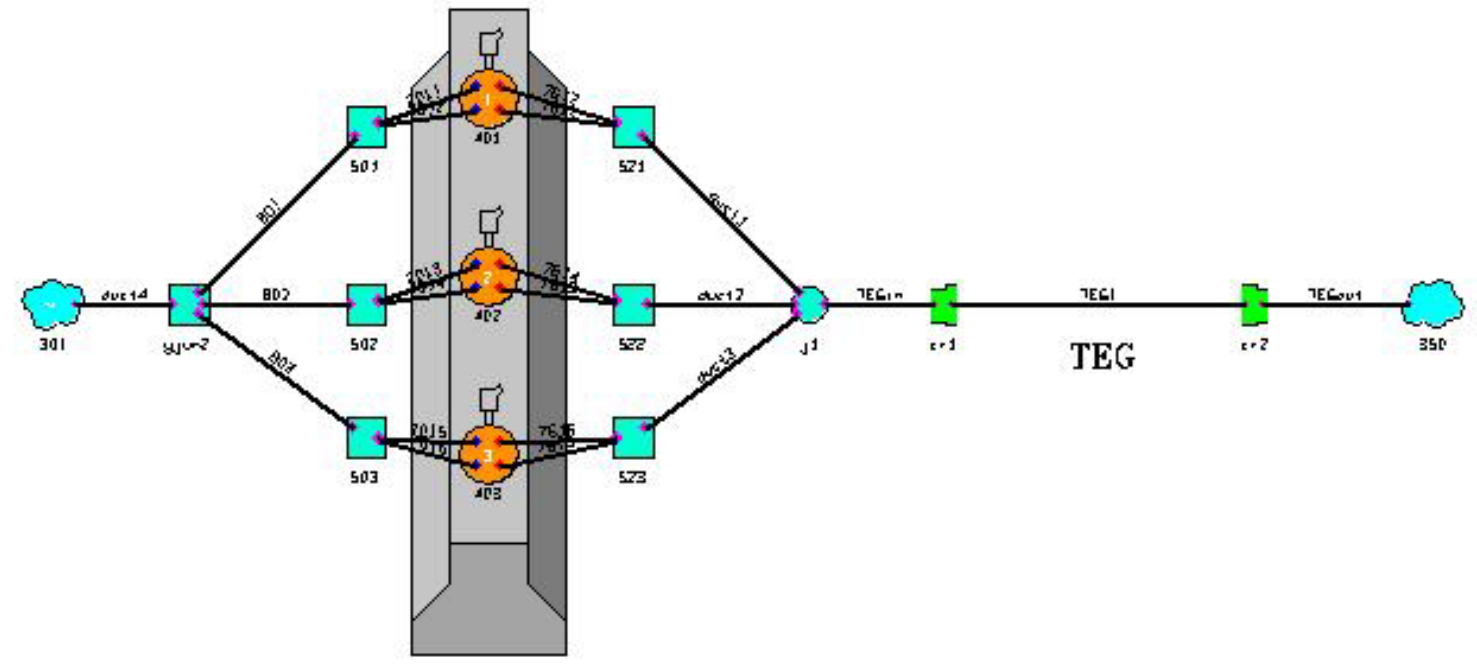

Figure 2.6. WAVE representation of 3-cylinder engine with one TEG.

\subsubsection{One TEG Unit Per Six Engine Cylinders}

Figure 2.7 shows the WAVE representation of this last configuration of interest. All six cylinders merge on the exhaust side into a single TEG duct. This case required the greatest cpu times since all cylinders had to be modeled to accurately predict the heat loading on the TEG and the output of the engine.

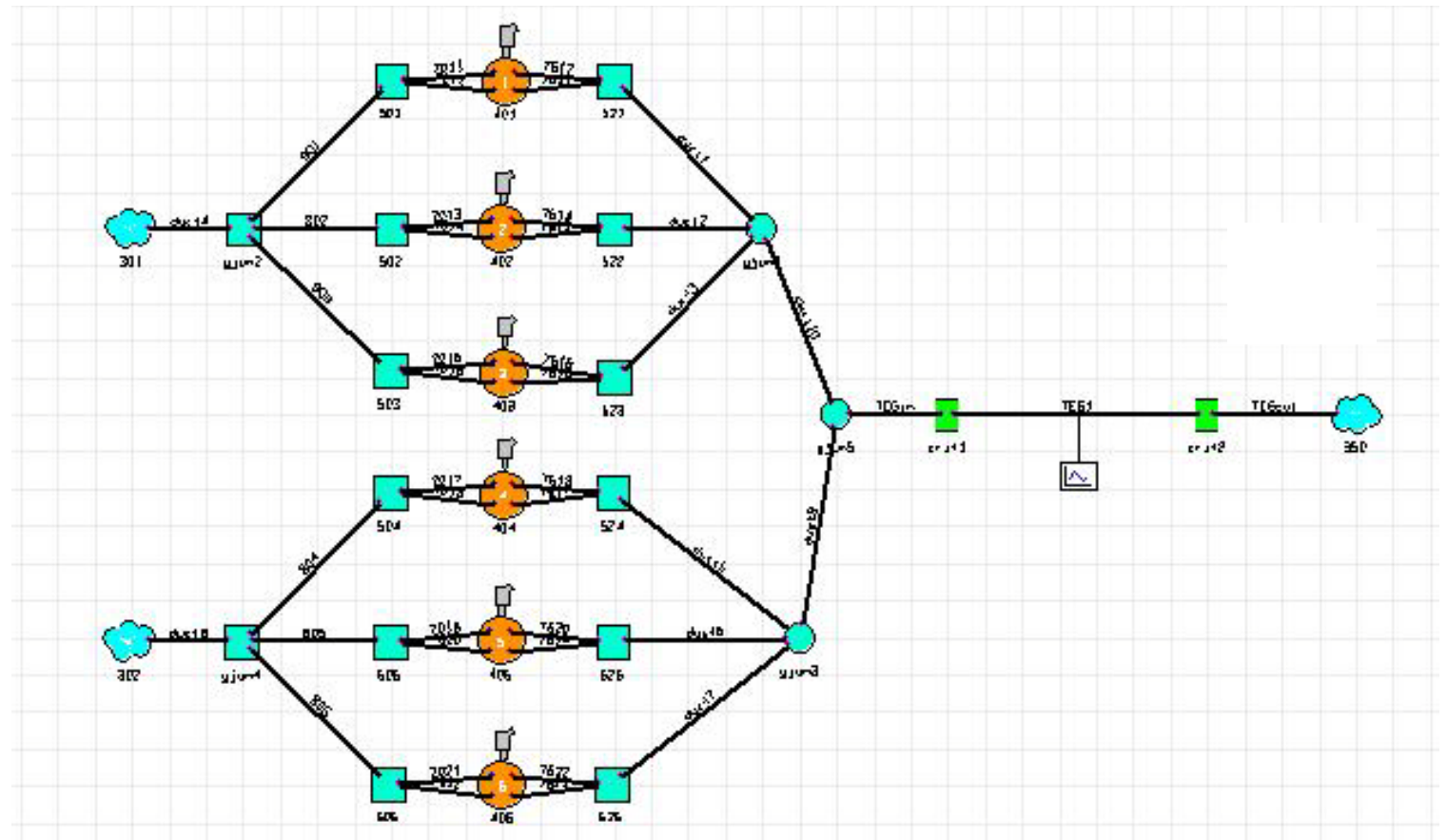

Figure 2.7. WAVE representation of 6-cylinder engine with one TEG. 


\subsection{Results}

\subsubsection{Validation for Base Engine}

The WAVE code was first used to simulate the baseline ISX production engine at the B62 operating point to validate model results for engine output. The detailed input included:

- intake and Exhaust Valve Lifts at one crank angle degree intervals

- intake and Exhaust Valve/Port Flow Coefficients at $1 \mathrm{~mm}$ valve lift intervals

- combustion Energy Release Profile

- base Engine Design Parameters (Bore, Stroke, Connecting Rod Length, Valve Diameters, Compression Ratio)

- centerline Lengths and Cross-Sectional Diameters of all Intake and Exhaust Ducting (Ambient to Valve Seat)

- operating Conditions (B62 “cruise point.” See Table 2.1)

The predicted value of engine BMEP was $210 \mathrm{psi}$. This is approximately $8.5 \%$ higher than the experimental value of 193.45 psi (listed in Table 2.1). A value within 10 percent of experimental data was considered acceptable. This is especially true in light of the somewhat idealized intake and exhaust system designs, which did not include the details of the turbocharger compressor and turbine. Flow and heat transfer losses throughout the actual engine's extensive induction and exhaust system components will typically reduce shaft output by approximately 10 percent. As such, this baseline level of engine output was considered well within the accuracy of engine simulation codes.

\subsubsection{Approach Used to Evaluate TEG Effectiveness}

Once it was determined that the code was simulating the base engine output in a reasonable manner, the single, three, and six cylinder TEG configurations were evaluated to determine which was the most effective in extracting exhaust heat energy. To simulate a "best case" scenario, several assumptions were incorporated into the engine-TEG system:

1. The engine piston, cylinder liners, and cylinder heat combustion chamber surfaces were near adiabatic. This was accomplished by reducing the gas side heat transfer coefficients by $99 \%$. This would be similar to using ceramic or other insulating coatings on these surfaces.

2. The exhaust ports and manifold were similarly assumed to be heat insulated to maximize the heat input into the TEG duct.

3. The TEG coolant temperature was assumed to be a constant value of 325 degrees Kelvin along its entire length. The coolant temperature will increase along its length, but this is of secondary importance in the resulting total exhaust heat extraction through the TEG structure.

4. The walls of the TEG are made up of two homogeneous layers. The inner gas-side layer is carbon steel, $3.175 \mathrm{~mm}(1 / 8$ ") thick, with a thermal conductivity of $48 \mathrm{~W} / \mathrm{m} / \mathrm{K}$; and the outside layer represents the P-N modules with a thickness of $11 \mathrm{~mm}$ and thermal conductivity of $3 \mathrm{~W} / \mathrm{m} / \mathrm{K}$. 
These assumptions allow for the maximum exhaust heat loading of the TEG. The following results thereby represent an upper bound to the energy that would be available for conversion to electrical power.

\subsubsection{Optimization of TEG Design}

Figure 2.27 contains the key dimensions for the baseline geometry of the single unit TEG. This configuration was used as the starting point to determine an "optimized" design for the single, three, and six cylinder cases, by simulating the engine operation at the B62 point for a range of TEG cross-section diameters and lengths. The primary output variable of WAVE (used as a measurement of the TEG effectiveness in these studies) was the total heat transferred per unit time through the walls of the TEG duct during an engine cycle, denoted "DHteg" (units of kilowatts) by the code. This variable is calculated within WAVE by integrating the gas-side heat flux, which depends on the instantaneous values of wall and gas temperatures and the instantaneous heat transfer coefficient, over the duct's internal surface area at each instant. The final number reported by WAVE represents a cycle-averaged value. The optimizations that follow are based on finding the maximum value of DHteg as a function of key design parameters and subject to certain constraints, such as maintaining engine output (BMEP), TEG packaging limitations, etc. BMEP is used as the measure of engine shaft output instead of brake torque because it normalizes the output with respect to engine displacement; i.e., it is independent of the number of engine cylinders.

The interior (exhaust gas-side) cross-section diameter was found to be the most important design variable for the TEG, because it has a direct effect on the engine output, as well as the amount of heat transferred from the exhaust gases. It was considered important in these studies to avoid any significant degradation of the ISX engine output by the introduction of the TEG in its exhaust stream; e.g., by increasing the exhaust flow restriction losses. Such a reduction in BMEP would ultimately have a detrimental impact on the engine's fuel efficiency and emissions by necessitating an increase in engine displacement to meet load requirements.

For the single cylinder case (one TEG per cylinder), Figure 2.8 shows the effect of changing the TEG interior (exhaust gas-side) cross-section diameter on both the engine BMEP (right-hand scale) and the total heat energy lost from the exhaust gases to the surrounding TEG wall surfaces per unit time during an engine cycle, DHteg (left-hand scale).

When the TEG diameter is reduced the BMEP does not change substantially until the diameter is less than 6.5 centimeters. This general behavior is expected, since reductions in the effective flow area of the engine exhaust system eventually lead to a significant restriction of the exhaust gas flow and an accompanying increase in the engine pumping work. Figure $\mathbf{2 . 8}$ also shows that DHteg increases with larger cross-section diameters. This follows from the concomitant increase in interior surface area within the TEG available for heat transfer from the exhaust gases. Although the heat transfer coefficient also increases as diameter is decreased (because of the associated increase in gas velocities) the dependence of the overall heat transfer in the TEG on surface area proves to be the dominant factor.

Based on these results, a diameter of $6.5 \mathrm{~cm}$ was chosen for the single-cylinder case. Although larger diameters would increase the value of DHteg, the average velocities through the TEG 
would decrease substantially, adversely affecting the performance of the turbocharger turbine located immediately downstream of the TEG duct. Therefore, the "optimum" TEG diameter was chosen to be the smallest value that does not diminish the engine output (BMEP) at the B62 operating point. Phase II studies will further refine these design parameter tradeoffs by modeling the full turbo-charge, wastegate, and EGR bypass system at both part and full load conditions. Similar TEG diameter optimization studies were carried out for the three- and six-cylinder configurations, resulting in diameters of $7.5 \mathrm{~cm}$ and $8.5 \mathrm{~cm}$, respectively.

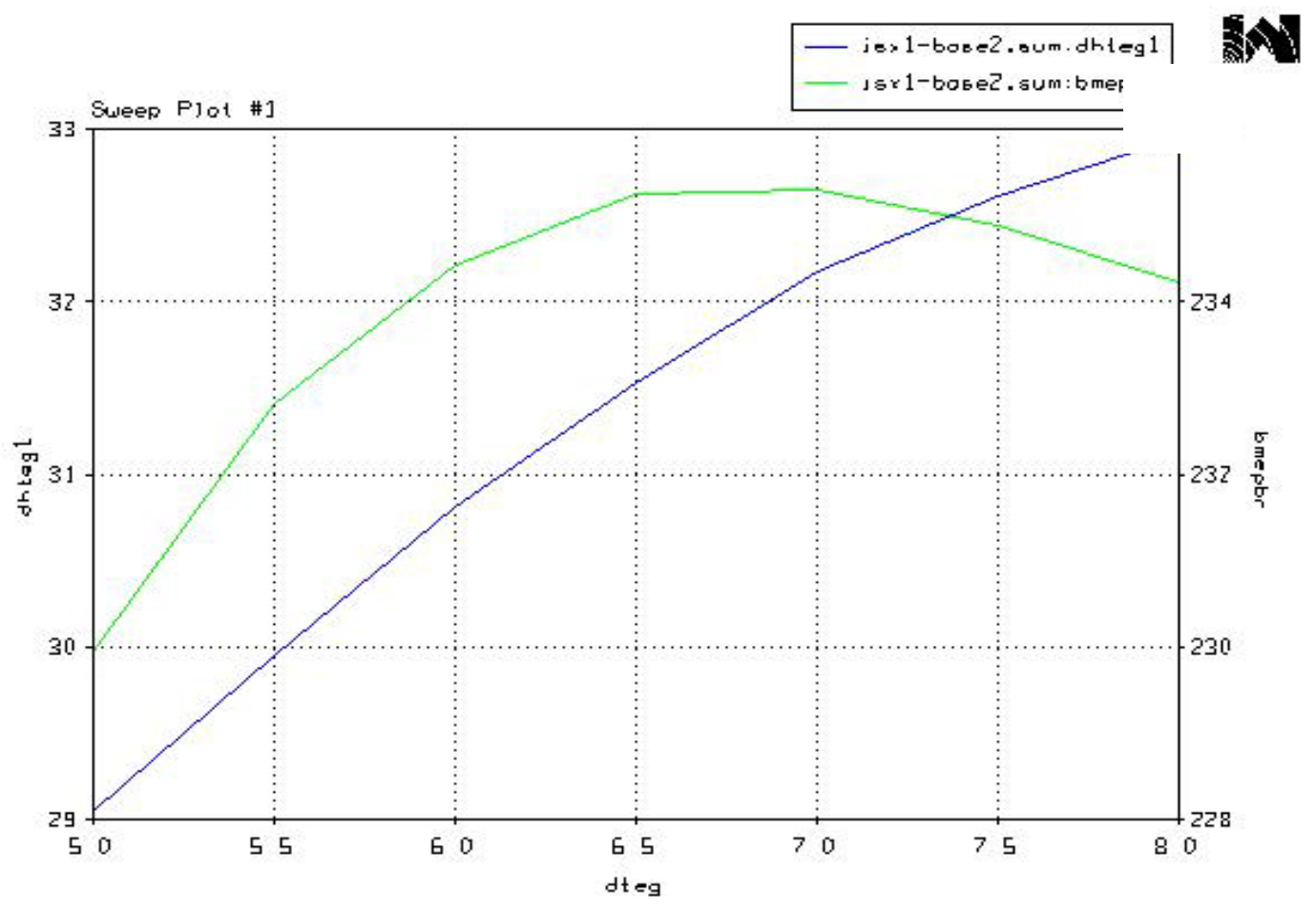

Figure 2.8. Effect of TEG effective diameter, single-cylinder case.

The other primary TEG design parameter is its axial length. As expected, the modeling results indicate a nearly linear increase in DHteg for lengths ranging from 0.5 meters to 2.5 meters. Within this range, the BMEP was not significantly affected. A final value of 1.5 meters was chosen for all three configurations due to estimated packaging limits for the ISX engine. Again, Phase II studies will further define this parameter. In the current feasibility study, we want to focus on the number and overall integration of the TEG(s) within the engine's exhaust system.

\subsubsection{Effects of Unsteady Flow Dynamics on TEG Heat Transfer}

It is important to note that the basic characteristics of the gas dynamics within the exhaust ducting and the TEG duct in particular are very different for the three configurations of interest. This is primarily because of the differing number of cylinders connected to the TEG. Each cylinder produces one exhaust "blowdown pulse" per engine cycle during the initial stages of the exhaust valve opening. Each of these pulses contains high pressure and temperature exhaust gases from the cylinder that will traverse each exhaust duct and be transmitted or reflected at 
duct transitions (e.g., changes in cross-sectional area). This in turn results in dynamic variations in the gas-side heat transfer coefficient. This is illustrated by showing the variation in the exhaust gas temperature, heat transfer coefficient, and gas velocity, respectively, in the TEG at its midpoint in length. The three curves shown in Figure 2.9 through Figure 2.11 are the results for the optimized geometries of the one-, three-, and six-cylinder cases.

Figure 2.9 shows distinct peaks in the gas temperatures for each of the cylinder blowdown events for the single-cylinder (one broad peak) and the six-cylinder (six peaks) cases. The threecylinder case has three primary peaks and three secondary peaks of lesser amplitude. The secondary peaks represent reflections of the primary waves at the ambient exit boundary. The abscissa is the engine crank angle for cylinder 1 , where zero crank angle degrees represents the TDC (top dead center) piston position at the beginning of the expansion stroke for cylinder 1 . For reference, the exhaust valve begins opening at 134 crank degrees.

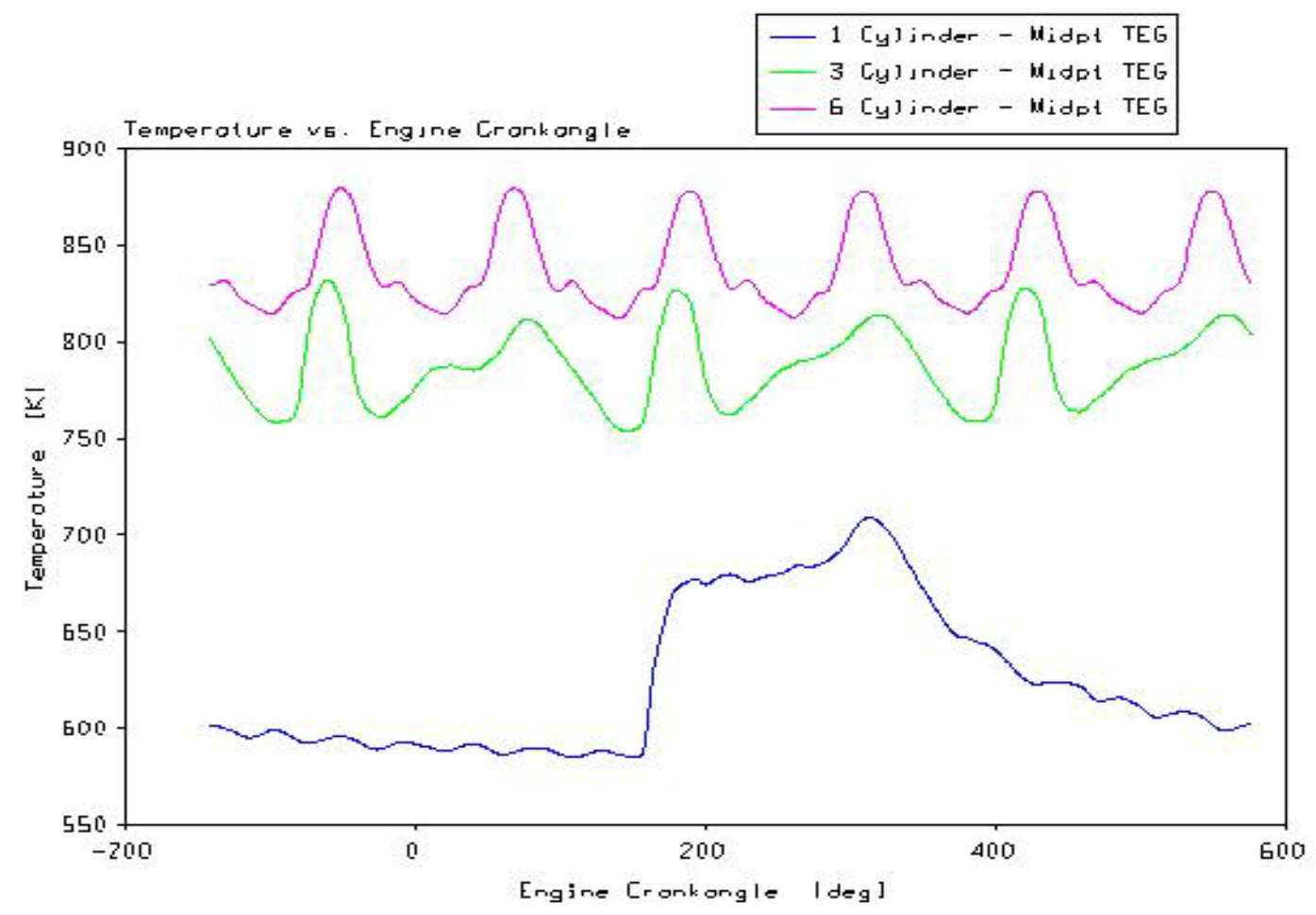

Figure 2.9. Exhaust gas temperatures at TEG midpoint.

Figure 2.10 shows the corresponding heat fluxes to the TEG walls at its midpoint location. The negative heatflux indicates heat transferred from the exhaust gases to the adjacent walls. Again, distinct primary peaks are observed and depend on the number of cylinders "feeding" the TEG. The single cylinder case has the higher amplitude heat flux because of the higher gas velocities (shown in Figure 2.11). The higher velocities are a result of the smaller cross-sectional area needed to expel the exhaust gases from a lesser number of cylinders. 


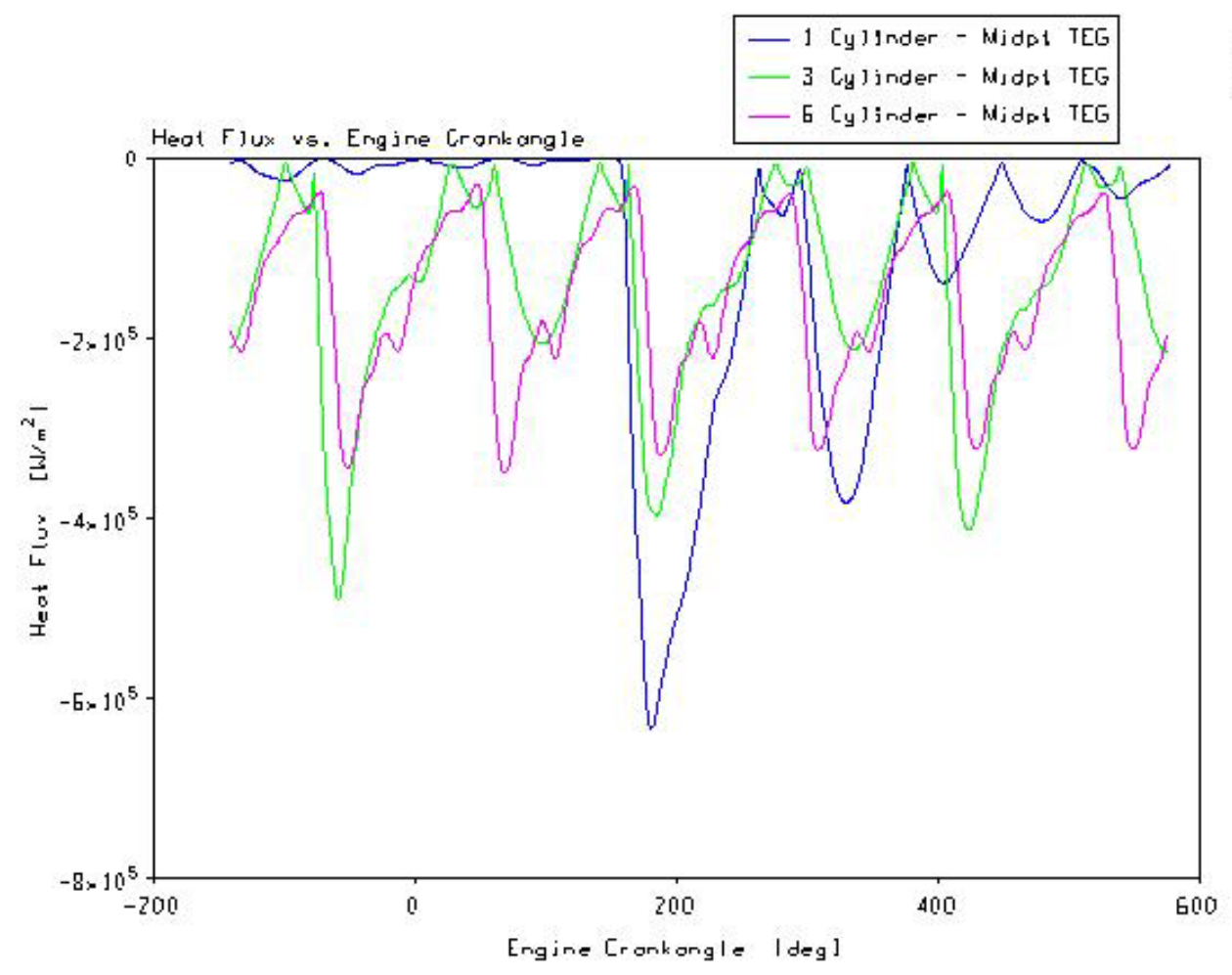

Figure 2.10. Heat flux at TEG midpoint.

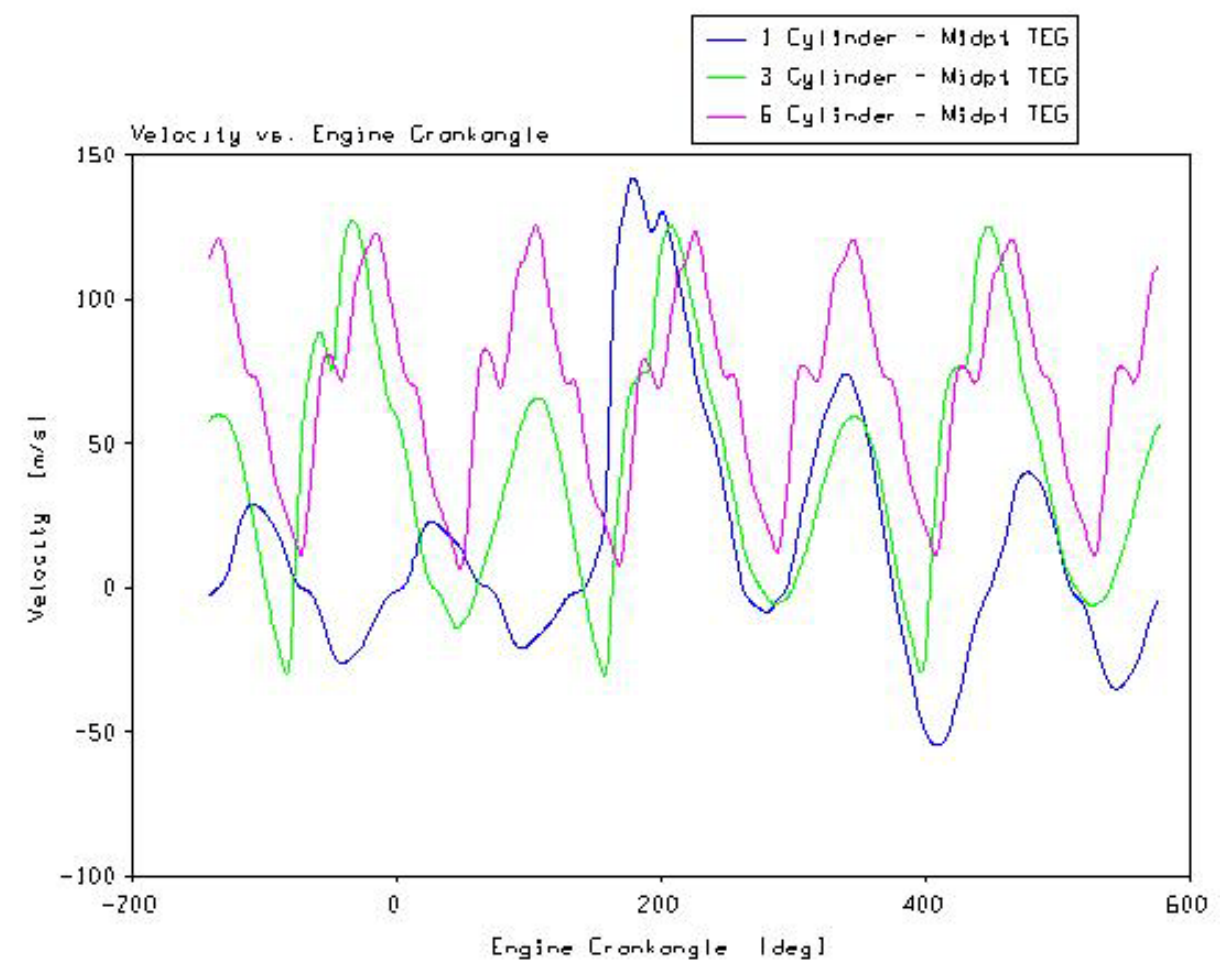

Figure 2.11. Gas velocities at TEG midpoint. 


\subsection{Overall Results}

Table 2.2 summarizes the overall results of this Phase I analytical study of the potential viability and benefits of the application of TEG technology to the ISX engine. All results are for the B62 operating point. The second column displays the resulting value of DHteg (average heat transfer rate from the exhaust gases to the walls of the single TEG in kWatts) for the one-, three-, and six-cylinder configurations. The third column breaks this number down to a per cylinder value. The final column gives the amount of energy transfer rate from the complete engine/TEG system (total of six, two, or one TEG respectively) for each of the configurations.

Table 2.2. Summary of overall results.

\section{Configuration (Optimized Geometry)}

One Cylinder per TEG Three Cylinders per TEG Six Cylinders per TEG

TEG Energy
Input Rate
[kWatts]

31.9

50.2

64.5

\section{Energy Input per Cylinder [kW/cylinder]}

31.9

16.7

10.8

\author{
Total TEG \\ Energy Input \\ [kWatts/engine]
}

191.4

100.4

64.5

The overall results indicate that more energy is extracted per cylinder, per engine cycle for the single cylinder configuration. This is because the total wetted gas-side surface heat transfer area of the TEG per cylinder is three and six times greater than for the three- and six-cylinder configurations. Also, the lower frequency of exhaust "events" per engine cycle for the singlecylinder case allows the interior walls more time to cool off between pulses and thereby provides a larger average temperature gradient to "drive" the transfer of energy from the exhaust gases to the TEG structure. Table 2.3 shows the time-average wall and exhaust gas temperatures at the inlet and exit of the TEG for each configuration. Both temperature values increase as the number of cylinders feeding the TEG increase.

Table 2.3. Average TEG gas and wall temperatures.

$\begin{array}{lcccc}\text { Configuration } & \begin{array}{c}\text { Wall Temp } \\ \text { (@TEG Inlet) }\end{array} & \begin{array}{c}\text { Gas Temp } \\ \text { (@TEG } \\ \text { [nlet) } \\ \text { [Degr K] }\end{array} & \begin{array}{c}\text { Wall Temp } \\ \text { [Degr K] }\end{array} & \begin{array}{c}\text { Gas Temp } \\ \text { [Degr K] }\end{array} \\ \text { [Degr K] } \\ \text { One Cylinder per TEG } & 708 & 856 & 597 & 634 \\ \text { Three Cylinders per TEG } & 798 & 884 & 662 & 711 \\ \text { Six Cylinders per TEG } & 831 & 898 & 731 & 785\end{array}$

Table 2.3 shows that one TEG unit per cylinder is more effective and preferred over the cases of one TEG per three- or six-cylinders and has profound implications for the development and testing of TEGs for application to internal combustion engines. Most importantly, a singlecylinder test engine, rather than a multi-cylinder (three or six) engine, is all that is necessary in the first development and test phase of the TEG. This in turn substantially lowers the time and cost to fabricate the prototype TEG designs and the associated testing. Moreover, the WAVE 
engine simulation can be used in conjunction with this hardware design and testing to further optimize the TEG for packaging as well as the function. Following the tests on a single-cylinder engine, WAVE can be calibrated to the experimental data and then used to project the TEG and engine performance on the full Cummins ISX six-cylinder engine

\subsection{Conclusions}

The objective of this Phase I analytical study was to quantify the potential benefits of thermoelectric generator (TEG) design alternatives in converting waste exhaust heat from the Cummins ISX, 15 liter, 6-cylinder, diesel engine into electrical energy. The studies are carried out for engine operating conditions most representative of those for an on-road, eighteenwheeled, Class 8 truck. To determine the maximum possible energy available for conversion, the TEG was located in the exhaust system immediately downstream of the engine's exhaust ports and manifold where it could serve as a possible replacement for the current engine's EGR (Exhaust Gas Recirculation) cooler. It was assumed that $100 \%$ of the exhaust gases pass through the TEG.

The study indicated several important results:

1) A greater amount of exhaust energy can be extracted through the TEG when each cylinder of the six-cylinder engine has its own separate TEG passage, as opposed to alternate designs having three- or six-cylinders "feeding" a single TEG unit. Comparing the most practical designs of one versus three cylinders per TEG unit, simulation results for the B62 (1500 rpm and $62 \%$ of full load) highway cruise operating point predict that the single-cylinder configuration allows over 90\% more energy extraction through the TEG unit per cylinder than the three-into-one design. For the full six-cylinder ISX engine, this indicates that 191.4 total kilowatts of exhaust energy could be extracted from the exhaust gases and is, therefore, available for conversion into electrical energy. A significant consequence of this finding is that the next stage of TEG design, development, and testing can be carried out using a singlecylinder engine test rig, as opposed to the full 15 liter, six-cylinder, engine. This would lead to substantial cost and time savings as TEG prototypes would be one-sixth smaller and therefore require fewer P-N modules. Also, the WAVE engine simulation code can then be calibrated to the single-cylinder test data and used to project the impact of each design iteration on the six-cylinder ISX engine.

2) Using liquid coolant, equivalent to that used in the engine cooling system (and assumed to be at a constant 325 degrees $\mathrm{K}$ ), to cool the outer walls of the TEG results in average exhaust gas-side wall temperatures in the range of approximately 600 - 700 degrees K.

\section{Heat Transfer Enhancements for Thermal Electric Power Generation}

\subsection{Heat Transfer Enhancement for TEPG}

Thermoelectric power generation refers to the coupling between the temperature gradient and the electric current. Heat transfer plays a very important role in the design of efficient TEPG systems. In order to produce compact and efficient TEPG systems, effective heat transfer enhancement concepts, which can be used to maintain temperature gradients on hot/cold plates of TE devices, must be evaluated. 
A simple analysis of the heat transfer through the TE legs will demonstrate the heat transfer rate needed in order to maintain the temperature on hot/cold plates of the TE system (Figure 3.1).

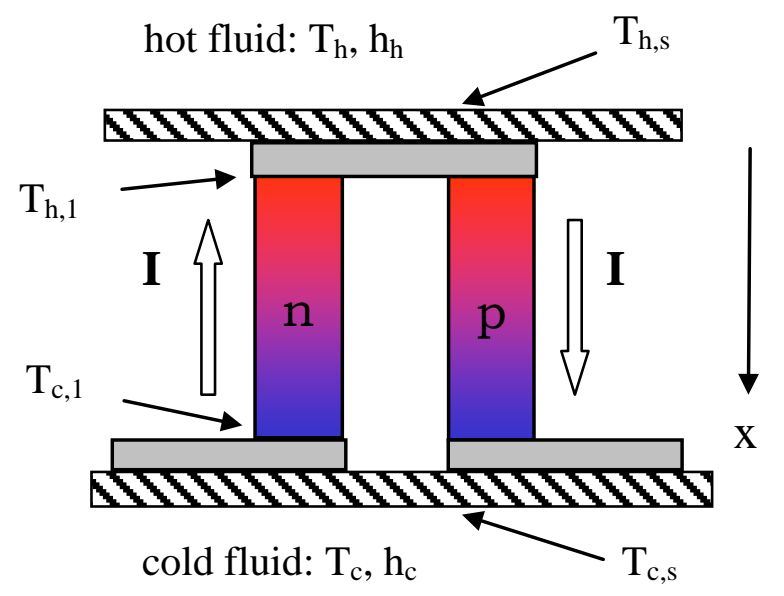

Figure 3.1. Heat transfer through a TE couple

Assuming the thermal conductivity of the TE material is constant ( $\kappa=2 \mathrm{w} / \mathrm{m}-K)$, heat loss from the sides of the TE legs is negligible, and $T_{h, 1}=T_{h, s}, T_{c, 1}=T_{c, s}$, the heat transfer rate will be

$$
q^{\prime \prime}=\kappa \frac{T_{h, s}-T_{c, s}}{l}
$$

where $l$ is the length of the TE legs. If the temperature difference is $600 \mathrm{~K}$, then

$$
\begin{aligned}
& q^{\prime \prime}=\kappa \frac{T_{h, s}-T_{c, s}}{l}=2.0 \frac{600}{l} \\
& q^{\prime \prime}=\kappa \frac{T_{h, s}-T_{c, s}}{l}=2.0 \frac{600}{0.012}=10 \mathrm{~W} / \mathrm{cm}^{2}, \text { for } l=12 \mathrm{~mm} \\
& q^{\prime \prime}=\kappa \frac{T_{h, s}-T_{c, s}}{l}=2.0 \frac{600}{0.001}=120 \mathrm{~W} / \mathrm{cm}^{2}, \text { for } l=1 \mathrm{~mm}
\end{aligned}
$$

Assuming the temperature of the hot working fluid is $100 \mathrm{~K}$ higher than the temperature on the hot plate, the heat transfer coefficients needed to transfer the above $q$ " to the TE hot plate will be

$$
\begin{aligned}
& q^{\prime \prime}=h\left(T_{h}-T_{h, s}\right) \\
& h=q^{\prime \prime} /\left(T_{h}-T_{h, s}\right)=100,000 / 100=1,000 \mathrm{~W} / \mathrm{m}^{2}-K, \text { for } l=12 \mathrm{~mm} \\
& h=q^{\prime \prime} /\left(T_{h}-T_{h, s}\right)=1,200,000 / 100=12,000 \mathrm{~W} / \mathrm{m}^{2}-K, \text { for } l=1 \mathrm{~mm}
\end{aligned}
$$


Assuming the temperature of the cold working fluid is $10 \mathrm{k}$ lower than the temperature on the cold plate, the heat transfer coefficients needed to transfer the above $q$ " from the TE cold plate will be

$$
\begin{aligned}
& q^{\prime \prime}=h\left(T_{h, s}-T_{h}\right) \\
& h=q^{\prime \prime} /\left(T_{h, s}-T_{h}\right)=100,000 / 10=10,000 \mathrm{~W} / \mathrm{m}^{2}-K, \text { for } l=12 \mathrm{~mm} \\
& h=q^{\prime \prime} /\left(T_{h, s}-T_{h}\right)=1,200,000 / 10=120,000 \mathrm{~W} / \mathrm{m}^{2}-K, \text { for } l=1 \mathrm{~mm}
\end{aligned}
$$

\subsection{Heat Transfer without Enhancement}

In this report, we consider air, water vapor, and liquid sodium as working fluids of transferring heat to the hot TE plate. The working pressure of the fluids is set to $1 \mathrm{bar}$, and the temperature is $1000 \mathrm{~K}$. The hydraulic diameter $D_{h}$ is adopted as $0.5 \mathrm{~cm}$.

The convection correlation of Nusselt number $N_{u}$ for fully developed laminar flow is [5]

$$
N_{u}=h D_{h} / \kappa=3.66
$$

air: $\kappa=0.0667 \mathrm{w} / \mathrm{m}-\mathrm{K}$, thus $\mathrm{h}=48.4 \mathrm{w} / \mathrm{m}^{2}-\mathrm{K}$

water vapor: $\kappa=0.0971 \mathrm{w} / \mathrm{m}-\mathrm{K}$, thus $\mathrm{h}=71.1 \mathrm{w} / \mathrm{m}^{2}-\mathrm{K}$

liquid sodium: $\kappa=59.7 \mathrm{w} / \mathrm{m}-\mathrm{K}$, thus $\mathrm{h}=43,700 \mathrm{w} / \mathrm{m}^{2}-\mathrm{K}$

The convection correlation of Nusselt number $N_{u}$ for fully developed turbulent flow is [5]

$$
N_{u}=h D_{h} / \kappa=0.023(R e)(4 / 5)(P r) 0.3
$$

where Re is Reynolds number based on $D_{h}$ and velocity v.

air: $\kappa=0.0667 \mathrm{w} / \mathrm{m}-\mathrm{K}, P_{r}=0.726, v=1.219 \times 10^{-4} \mathrm{~m}^{2} / \mathrm{s}$,

$$
\begin{aligned}
& \mathrm{h}=1063.8 \mathrm{w} / \mathrm{m}^{2}-\mathrm{K}(\operatorname{Re}=30,000, \mathrm{v}=731.4 \mathrm{~m} / \mathrm{s}) \\
& \mathrm{h}=253.7 \mathrm{w} / \mathrm{m}^{2}-\mathrm{K} \quad(\operatorname{Re}=5,000, \mathrm{v}=121.9 \mathrm{~m} / \mathrm{s})
\end{aligned}
$$

water vapor: $\kappa=0.0971 \mathrm{w} / \mathrm{m}-\mathrm{K}, P_{r}=0.0971, v=1.735 \times 10^{-4} \mathrm{~m}^{2} / \mathrm{s}$,

$$
\begin{aligned}
& \mathrm{h}=1644.6 \mathrm{w} / \mathrm{m}^{2}-\mathrm{K} \quad(\operatorname{Re}=30,000, \mathrm{v}=1041 \mathrm{~m} / \mathrm{s}) \\
& \mathrm{h}=392.2 \mathrm{w} / \mathrm{m}^{2}-\mathrm{K} \quad(\operatorname{Re}=5,000, \mathrm{v}=173.5 \mathrm{~m} / \mathrm{s})
\end{aligned}
$$

liquid sodium: $\kappa=59.7 \mathrm{w} / \mathrm{m}-\mathrm{K}, P_{r}=0.0037, v=2.285 \times 10^{-7} \mathrm{~m}^{2} / \mathrm{s}$,

$$
\begin{aligned}
& \mathrm{h}=72,618 \mathrm{w} / \mathrm{m}^{2}-\mathrm{K}(\operatorname{Re}=30,000, \mathrm{v}=1.37 \mathrm{~m} / \mathrm{s}) \\
& \mathrm{h}=62,781 \mathrm{w} / \mathrm{m}^{2}-\mathrm{K}(\operatorname{Re}=5,000, \mathrm{v}=0.228 \mathrm{~m} / \mathrm{s})
\end{aligned}
$$

Now, we consider removing the heat from the TE cold plate. Liquid water will be chosen as working fluid. The working pressure of the fluid is set to $1 \mathrm{bar}$, and the temperature is $290 \mathrm{~K}$. The hydraulic diameter $D_{h}$ is adopted as $0.5 \mathrm{~cm}$. 
The above convection correlation of Nusselt number $N_{u}$ for fully developed turbulent flow will be used to calculate the heat transfer coefficient.

water at 1 bar and 290K: $\kappa=0.598 \mathrm{w} / \mathrm{m}-\mathrm{K}, P_{r}=7.56$, and $v=1.08 \times 10^{-6} \mathrm{~m}^{2} / \mathrm{s}$,

$$
\begin{aligned}
& \mathrm{h}=19,262.5 \mathrm{w} / \mathrm{m}^{2}-\mathrm{K}(\operatorname{Re}=30,000, \mathrm{v}=6.48 \mathrm{~m} / \mathrm{s}) \\
& \mathrm{h}=4594 \mathrm{w} / \mathrm{m}^{2}-\mathrm{K}(\operatorname{Re}=5,000, \mathrm{v}=1.08 \mathrm{~m} / \mathrm{s})
\end{aligned}
$$

From the above calculations, it can be seen that there are large gaps between the needed heat transfer coefficients and the available ones, even if the velocity is impractically high. Thus, there will be a major challenge of maintaining the specified temperatures on the hot and cold plates.

\subsection{Summary}

We first calculated the heat transfer coefficients needed in order to maintain $900 \mathrm{~K} / 300 \mathrm{~K}$ on hot/cold plate of the TE devices in limiting cases. We then assessed heat transfers without enhancement measures for different working fluids. In the last step, we assessed enhancement methods of heat transfer.

This assessment shows that it will be a substantial challenge to transfer sufficient heat to or from the hot/cold plates of the TE system, especially when the length of TE legs is very small and/or the thermal conductivity of the leg material is not small enough.

Ribbed and dimpled surfaces can increase heat transfer approximately $3 \sim 4$ times over the original value. Other enhancement efforts and heat transfer optimizations need to be done in the next step of designing a TE device.

\section{Selection of Thermoelectric Materials and Generator Design}

The new high efficiency materials that have recently emerged from our laboratories at Michigan State University remain under development. A thermoelectric device is made of two semiconductor legs: N-type and P-type. The Kanatzidis and Hogan groups at MSU have been working on the $\mathrm{AgPb}_{\mathrm{m}} \mathrm{SbTe}_{2+\mathrm{m}}$ system (LAST-m for lead-antimony-silver-tellurium) which we plan to use for the purposes of this project. The MSU materials are bulk systems made of inexpensive elements and are subject to large-scale production. In the past three years we have selected the LAST-18 composition. When doped appropriately, it is capable of achieving high ZT $(\sim 1.7$ at $700 \mathrm{~K})$ at high temperatures suitable for high efficiency heat to electrical energy conversion applications.

The N-type material $\mathrm{Ag}_{\mathrm{x}} \mathrm{Pb}_{18} \mathrm{Sb}_{1.2} \mathrm{Te}_{20}(\mathrm{x}=0.4-0.9)$ has been under intense investigation in order to optimize its performance at large scale preparation. We have selected the compositions with $\mathrm{x}=0.43$ to optimize our synthesis and processing profiles. We are in a position to produce 100 gram low resistivity ingots with strong mechanical properties that allow us to cut and produce TE legs. We have also begun to use these ingots as feed material for grinding into fine powders of specified size for powder metallurgy processing (i.e. hot pressing). The lack of a hot press at MSU has hampered the speed at which we could do this work. We plan to acquire a hot press with funds obtained from Phase II of this project. 
We have found a very good heating/cooling profile for LAST-18 of the composition $\mathrm{Ag}_{0.43} \mathrm{~Pb}_{18} \mathrm{Sb}_{1.2} \mathrm{Te}_{20}$. The ingot looks and behaves well in terms of integrity and resistance. This ingot was in vertical position during the entire cooling process. The photo below (Figure 4.1) shows the appearance of this ingot. The different reflections and shades on the surface of the sliced "coins" are not different phases but correspond to reflection from different crystal orientations.

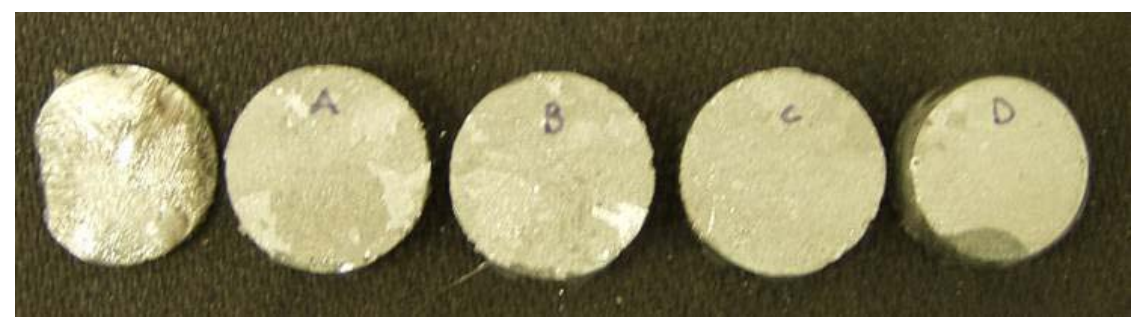

Figure 4.1. Coins sliced from a $100 \mathrm{~g}$ ingot and numbered from A (top) to D (bottom) for further investigations. The thickness of each slice is $5 \mathrm{~mm}$. The average resistance of this $100 \mathrm{~g}$ ingot was 0.0013 ohms. This corresponds to a room temperature conductivity of $\sim 1400-1800 \mathrm{~S} / \mathrm{cm}$.

Our thermal stability studies suggest that the highest safe operating temperature expected from devices incorporating this material would approximately $800 \mathrm{~K}$. Beyond this temperature, one needs to worry about long-term stability and diffusion leading to degradation of properties. Our materials are therefore suitable for devices operating in the temperature range of 300-700 K.

\subsection{High Performance TE P-Type Materials by Modification of $\mathrm{AgPb}_{18} \mathrm{SbTe}_{20}$ Materials (LASTT)}

High ZT P-type materials were successfully achieved by adding $\mathrm{Sn}$ in the $(\mathrm{Ag} / \mathrm{Pb} / \mathrm{Sn} / \mathrm{Sb} / \mathrm{Te})$ system. These materials are called LASTT (lead antimony silver tellurium tin). The data shown below (Figure 4.2) were obtained from various LASTT compositions exhibiting very promising TE properties. Further optimization of these materials will continue in Phase II to achieve the best possible TE properties that are suited for the high engine temperatures available. In general, the thermal conductivity achievable with these materials is very low. For example, the lattice thermal conductivity of $\sim 0.7 \mathrm{~W} / \mathrm{mK}$ is much lower than that observed in the N-type LAST materials $(1.2 \mathrm{~W} / \mathrm{mK})$. These P-type materials are also strongly nanostructured with extensive compositional fluctuations at the nanoscale. The composition $\mathrm{Ag}_{0.5} \mathrm{~Pb}_{7} \mathrm{Sn}_{3} \mathrm{Sb}_{0.2} \mathrm{Te}_{12}$ will be selected and implemented into the multi-leg modules. Below are some of the most relevant results. 
$\mathrm{Ag}_{0.5} \mathrm{~Pb}_{7} \mathrm{Sn}_{3} \mathrm{Sb}_{0.2} \mathrm{Te}_{12}(\mathrm{JAE79P132-B})$
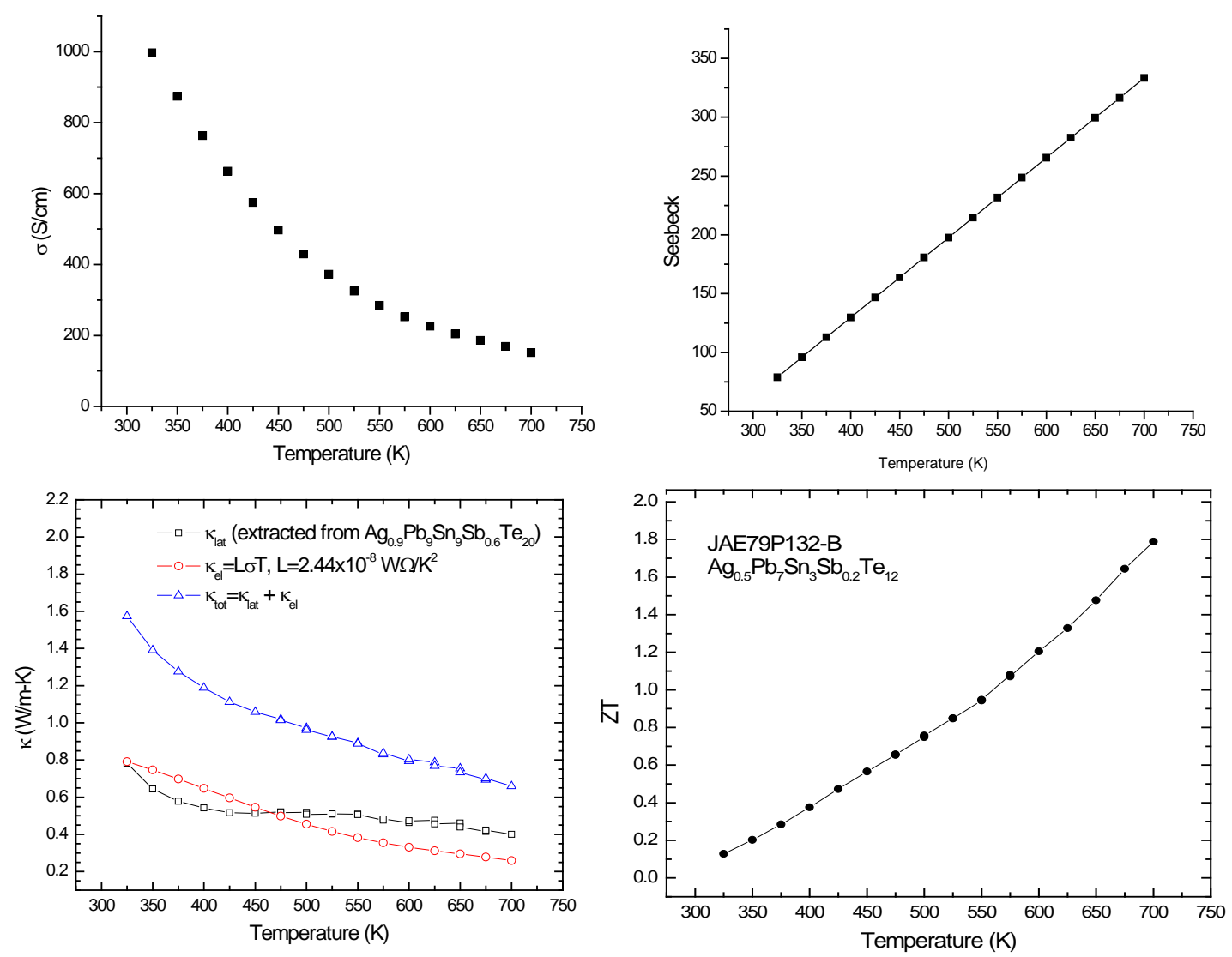

Figure 4.2. Electrical conductivity, Seebeck coefficient, thermal conductivity and $\mathrm{ZT}$ plot for $\mathrm{Ag}_{0.5} \mathrm{~Pb}_{7} \mathrm{Sn}_{3} \mathrm{Sb}_{0.2} \mathrm{Te}_{12}$ as a function of temperature.

The ZT values routinely exceed 1 at $700 \mathrm{~K}$. All of these samples have good reproducibility and good homogeneity and should be able to be reproduced easily at Tellurex even on a large scale of $1000 \mathrm{~g}$.

With further materials research we anticipate that within the next four years we will have developed a new generation of both N-type and P-type TE materials with ZT 2 and hopefully 3 at $600 \mathrm{~K}$. We are learning how to further reduce the thermal conductivity through better control of the nanostructuring and through raising the average power factor.

\section{Electrical Energy Utilization}

The electrical systems and modeling team envisions the recovery of engine waste heat in large OTR trucks with the primary purpose of redirecting this energy to the vehicle's wheels. Recovery of waste heat and its immediate delivery to the vehicle wheels turns this concept into a thermal power split hybrid. This is a micro hybrid to be precise. The overall architecture of the thermal power split hybrid is shown as Figure 5.1. 


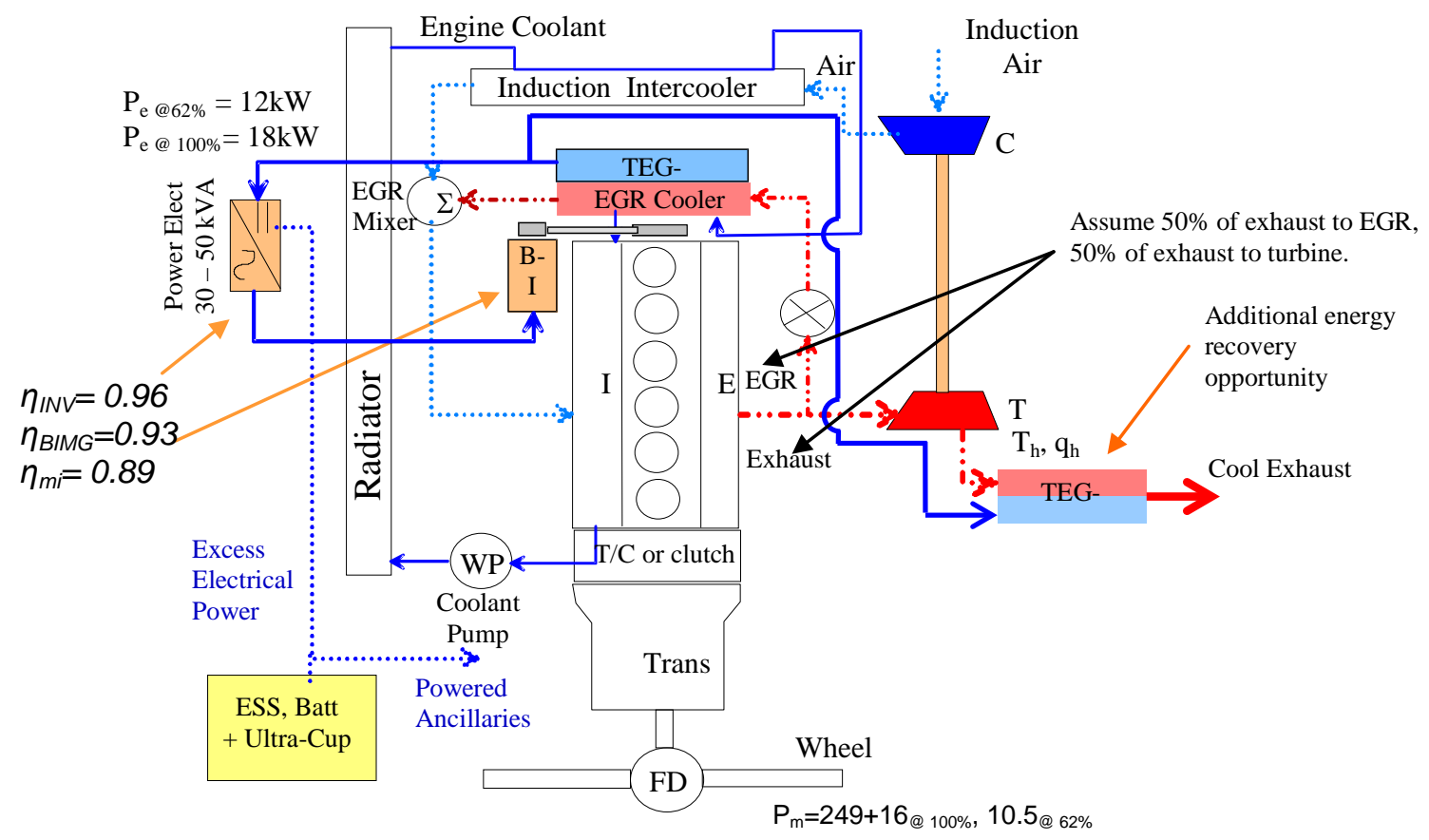

Figure 5.1. Architecture of the thermal power B62 cruising mode.

In the system shown in Figure 5.1, a fraction of the heat rejected via the engine exhaust will be returned to the engine induction port via an exhaust gas recirculation (EGR) loop. Opportunity for energy scavenging exists in the EGR loop because a conventional EGR will incorporate an EGR cooler to chill the exhaust gas to near ambient conditions. Rather than reject this heat through the radiator, the presence of a thermoelectric generator will use the presence of a thermal gradient to convert a fraction of this rejected heat directly into electrical power. The remainder of the engine-rejected heat is delivered in the conventional manner to the turbine side of the turbo charger and from there to the exhaust system.

The recovered waste heat from the EGR loop is matched to the hybrid drive system at a nominal potential of $144 \mathrm{Vdc}$. At this voltage level two important attributes are realized: 1) it is economical to process up to $20 \mathrm{~kW}$ or more using today's power electronics and electric machine technology and 2) the required number of total TEG couples at $144 \mathrm{~V}$ is less than that of a $42 \mathrm{~V}$ nominal electrical distribution bus voltage. The net value of both benefits is that the overall warranty is improved because fewer couples are series connected and moreover the need for excessive parallel paths is minimized.

With the availability of a $144 \mathrm{~V}$ nominal dc bus, the next step is to process this power and to deliver it to the vehicle wheels. Figure 5.2 illustrates the electronic power processor considered for this application. In this system we consider the available heat energy at $100 \%$ engine output (1) and at $62 \%$ engine output (2), i.e. steady cruise. For these two cases and for the levels of exhaust heat considered: 


$$
\begin{aligned}
& P_{\text {elec } 100 \%}=\frac{P_{E G R}}{2} \eta_{\text {TEG }} \\
& P_{\text {elec } 100 \%}=\frac{323 \mathrm{~kW}}{2}(0.11)=17.71 \mathrm{~kW} \\
& P_{\text {elec } 62 \%}=\frac{217}{2}(0.11)=11.94 \mathrm{~kW}
\end{aligned}
$$

Furthermore, we assume the U.S. DOE FreedomCAR targets for power electronics and electric machines have efficiency values of 0.96 and 0.93 . This yields a gross motor-inverter efficiency of 0.89 . Thermoelectric power of $18 \mathrm{~kW}$ (100\% engine output) and $12 \mathrm{~kW}$ (62\% output) is available at $144 \mathrm{~V}$ nominal at the power electronics inverter rails. The electrical power processed by the power electronic bridge and the energy conversion by the belt-connected integrated starter generator (ISG) results in the mechanical power of $16 \mathrm{~kW}$ when at $100 \%$ engine output and 10.5 $\mathrm{kW}$ at $62 \%$ engine output, while being delivered into the engine crankshaft. This recovered power is then available directly to the driven wheels via the vehicle driveline.

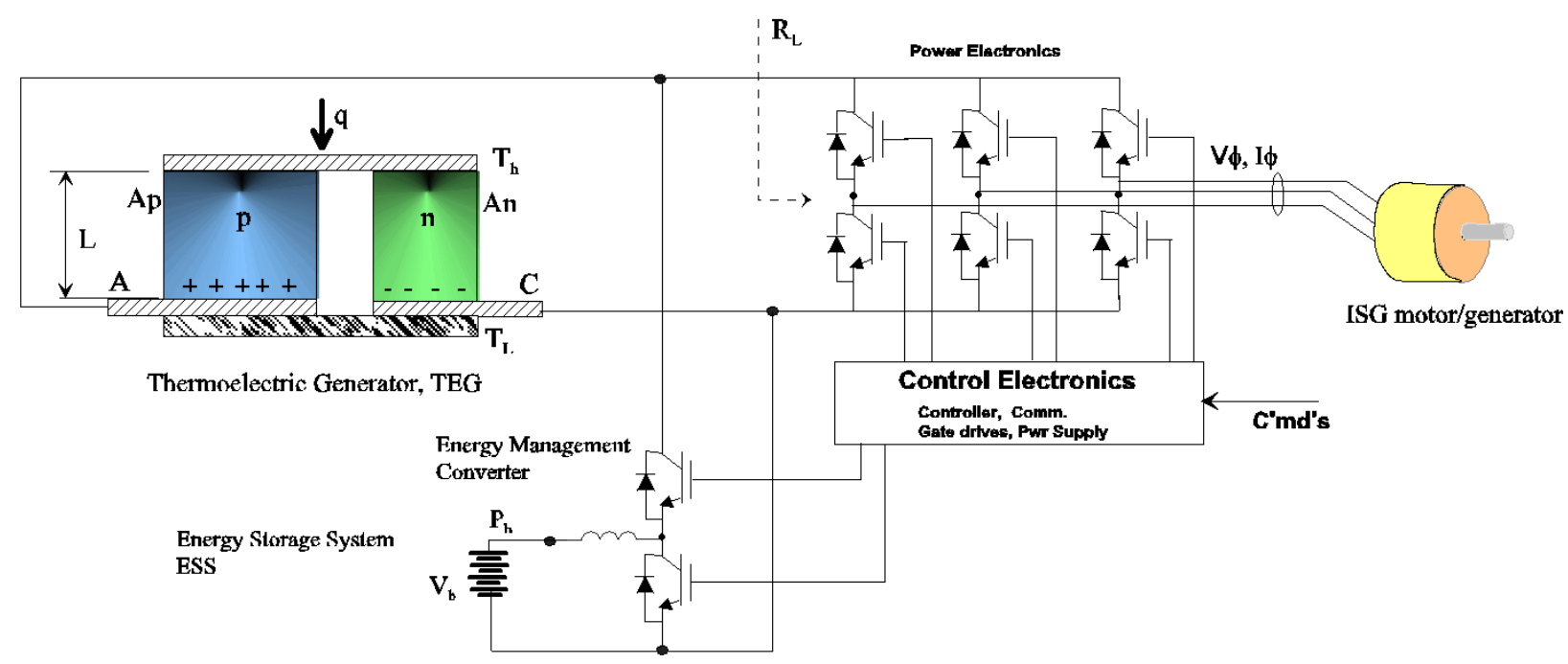

Figure 5.2. Belt integrated starter alternator configuration for OTR Truck. 

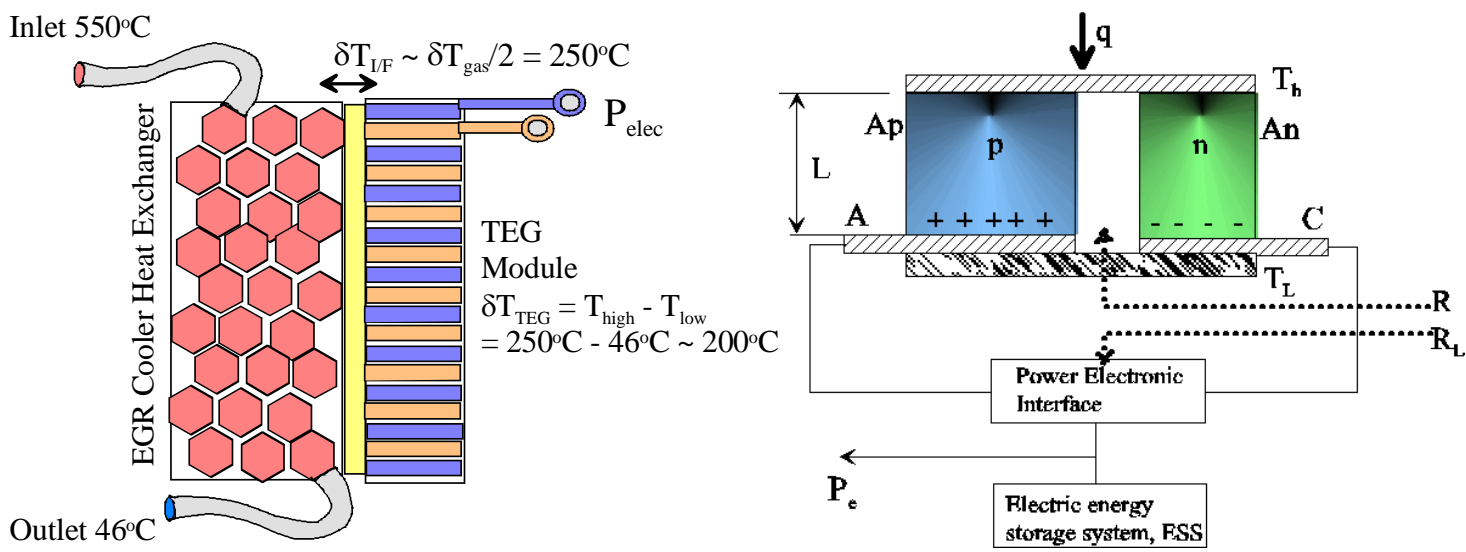

Figure 5.3. TEG couple and heat exchanger package illustration.

In previous updates the thermodynamic performance of the TEG has been examined and described. Recent work resulted in a desired voltage level of $144 \mathrm{~V}$ for the energy recirculation path. Figure 5.3 illustrates the TEG couple and its package environment in a heat exchanger coupled to the EGR gas flow. Based on preliminary Seebeck coefficient values of $145 \mu \mathrm{V} / \mathrm{K}$ couple it is easy to show that the required number of series couples (42V and at $144 \mathrm{~V}$ ) are 1141 and 3913 respectively when the thermal gradient is $573-319^{\circ} \mathrm{C}$. The packaged TEG would therefore consist of a single string of 3913 couples in a $144 \mathrm{~V}$ nominal system or 4 strings in parallel of 1141 couples in a $42 \mathrm{~V}$ system for a total of 4564 couples.

What further aggravates the voltage selection is the need to minimize couple interconnect resistances. For the stated power level of $13.4 \mathrm{~kW}$ and a matched load case achieved by simply shorting the string of couples together would mean that the total interconnect resistance of the string would match the couple internal resistance, $R_{m}$ where the subscript " $m$ " refers to the full module.

$$
\begin{aligned}
& R_{I C 42 V}=\frac{V_{o c}^{2}}{4 P_{\max }}=\frac{42^{2}}{4(13.4)} m \Omega=32.9 \mathrm{~m} \Omega \\
& R_{I C 144 V}=\frac{144^{2}}{4(13.4)} m \Omega=386.8 m \Omega
\end{aligned}
$$

For the given number of series couples, Nc, for each voltage level the individual interconnect resistance in each case is interestingly approximately the same.

$$
\begin{aligned}
& R_{\text {IC 42 couple }}=\frac{4 R_{I C 42 V}}{N_{c 42}}=\frac{4(32.9)}{1141} m \Omega=115 \mu \Omega \\
& R_{I C 144 \text { Couple }}=\frac{R_{I C 144}}{N_{c 144}}=\frac{386.8}{3913} m \Omega=99 \mu \Omega
\end{aligned}
$$


The final level of activity includes the development of a simulation model for the TEG that can be used in various electronic simulation programs such as Ansoft Simplorer, Orcad PSPICE, and other circuit simulation software packages. We elected to use a coupled thermo-electric model consisting of a pure electronic branch that models the Seebeck potential (or Peltier effect in the event of a TE cooler) and the couple internal electronic resistance, $\mathrm{R}_{\mathrm{m}}$, The second branch of the TEG model represents the heat flux path through the material thermal conductivity paths, the Peltier cooling and Joule heating effects.

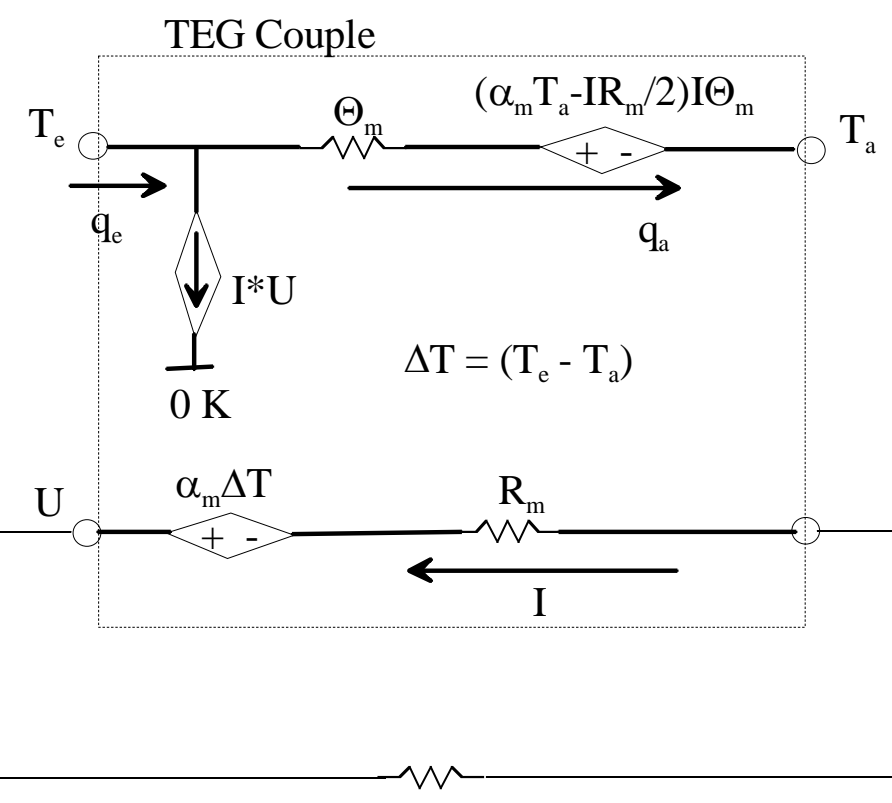

Figure 5.4. TEG electronic simulation model.

The couple within the TEG model exists between two thermal potentials; one side is the emitter and the second side is the absorber. These roles are reversed in the case of a TE cooler. The hot side of the TE generator is labeled "emitter" and the cooler side "absorber". The heat flux qe (W from emitter) and $\mathrm{q}_{\mathrm{a}}$ (W into absorber) modeled are shown in Figure 5.4.

In the TEG model of Figure 5.4, a thermal gradient from emitter to absorber sides of the couple gives rise to the development of a Seebeck generator having an open circuit and terminal voltage according to (5).

$U_{o c}=\alpha_{m}\left(T_{e}-T_{a}\right)=\alpha_{m} \Delta T$

$U=\alpha_{m} \Delta T-R_{m} I$

In the above model, $\mathrm{I}<0$ represents generator action and $\mathrm{I}>0$ is $\mathrm{TE}$ cooler action.

The loop equations for the thermal and electronic branches of the equivalent circuit (Figure 5.4) result in the thermodynamic equations of the TEG. 


$$
\begin{aligned}
& \Delta T=\Theta_{m} q_{a}+\left(\alpha_{m} T_{a}-\frac{I R_{m}}{2}\right) I \Theta_{m} \\
& q_{a}=\frac{\Delta T}{\Theta_{m}}-\alpha_{m} T_{a}(-I)+\frac{I^{2} R_{m}}{2} \\
& q_{a}=\frac{\Delta T}{\Theta_{m}}+\alpha_{m} T_{a} I+\frac{I^{2} R_{m}}{2}
\end{aligned}
$$

In this development (6) is the form of the heat flux into the absorber side due to thermal conductivity where $\alpha_{\mathrm{m}}$ is thermal resistance $=1 / \mathrm{K}$, followed by Peltier cooling of the absorber and lastly by Joule heating.

The performance at the emitter side is obtained as follows.

$$
\begin{aligned}
& q_{e}-I U=q_{a} \\
& q_{e}=q_{a}+\alpha_{m} \Delta T-I^{2} R_{m} \\
& q_{e}=\frac{\Delta T}{\Theta_{m}}+\alpha_{m} T_{e} I-\frac{I^{2} R_{m}}{2}
\end{aligned}
$$

Equations (5), (6) and (7) completely model the TEG for any thermal gradient provided the appropriate model parameters are available.

The next step in this activity is to obtain suitable values for the TEG couple in terms of Seebeck coefficient, $\Theta_{\mathrm{m}}$, internal resistance, $\mathrm{R}_{\mathrm{m}}$, and thermal resistance, $\alpha_{\mathrm{m}}$. Also, characteristic parameters of the TEG can be obtained through evaluation of these same equations for $\Delta \mathrm{T}_{\max }$, $\mathrm{I}_{\max }$, and $\mathrm{U}_{\max }$ in terms of the dimensionless figure of merit, $\mathrm{Z}$. These derivations remain the subject of future work, including the development of the required model parameters, simulation of the model, and comparison to experimental work.

\section{Summary}

From the detailed numerical modeling included in Phase I, the calculated efficiency improvements are as follows: (i) 1 TEG/Cylinder 6.2\%, (ii) 2 TEGs/6 Cylinders 4.0\% and (iii) 1 TEG/6 Cylinders: 2.8\%. Additional benefits are expected with an integrated starter generator (two times the efficiency of current alternators) and operation at greater than cruise power. Costs of the TEG system can be low due to the synergy with current hybrid vehicles for power electronics and the use of inexpensive TE materials. Another benefit of the design generated in Phase I is that the immediate use of electrical energy eliminates storage issues.

TE materials operating from 400-800K are expected to dominate this application. The material property and fatigue characterization are of critical importance for transient operation of this device.

The single-cylinder option (1 TEG/Cylinder) is the best for a Phase II demonstration system, with an efficiency that is superior to all other configurations. The numerical modeling work 
coupled with measurements on the Phase II demonstration system will give us the opportunity to develop and verify detailed transient heat transfer models for pulsate, three dimensional, compressible flow of exhaust through the TEG system. Tested computational tools exist for a scale-up to multi-cylinder applications. The costs associated with the single-cylinder option allow the evaluation of alternatives that could not be studied using the same resources in a multicylinder configuration.

During Phase II, iterative studies will be conducted to determine optimum material combinations. The powder processing and hot pressing techniques for TE leg fabrication will be developed and optimized. The thermal and mechanical properties of the TE materials will be determined, including the fracture strength and the fracture toughness, along with the thermalmechanical fatigue behavior (which is critical for the long term stability of the TE materials). In addition, diffusion barriers and coatings will be developed to suppress sublimation of the TE materials over the range of operating temperatures. Phase II will also entail the development of a detailed power electronic system design along with the evaluation of the dynamic response of electrical system as ÄT changes.

A scale model demonstration unit with an efficiency gain of $5 \%$ is a reasonable 5 year goal. If the materials that have an energy conversion efficiency of $18 \%$ for this temperature range can be developed then a $12.4 \%$ BSFC improvement would be predicted.

\section{Phase II}

\section{Introduction}

The previous Phase I effort has described the technology barriers to overcome to facilitate the successful implementation of thermoelectric technology to extract electric energy from the exhaust gases of an over the road Class 8 diesel powerplant. In Phase I, we evaluated the materials developed at MSU (Kanatzidis, Hogan and Schock). We also considered current materials and module designs of one of our project partners, NASA's Jet Propulsion Laboratory (JPL). The MSU materials were evaluated in conjunction with engine simulations and TEG thermal analysis. Using a different set of materials (skutterudites), JPL has built high efficiency thermoelectric generators with proven reliability and efficiency. The JPL experience provides us the opportunity to leverage our effort with over 50 years worth of experience in the design, construction and cost analysis related to TEGs. It is critical to calculate the temperature gradients available in the exhaust stream while maintaining the performance of the engine.

Much work has been devoted to developing thermoelectric materials, calculating theoretical power outputs and researching the most cost-effective applications for these devices. However, there have been very few experiments performed that put these theoretical concepts to the test by measuring the actual power generated by the thermoelectric modules in a semi-realistic environment. In Phase II our work concentrates on developing the methods to build and test thermoelectric generators. These methods include material synthesis, powder processing, hot pressing, couple fabrication, modules fabrication and generator testing. The goal of the generator testing was to design, fabricate and test a $50 \mathrm{~W}$ and a $100 \mathrm{~W}$ thermoelectric generator and thus verify the feasibility of using high-temperature thermoelectric generators for salvaging energy 
from waste heat. In addition to fabricating and testing a thermoelectric generator, we continued parallel studies of new material research, powder processing techniques, mechanical property characterization and power electronics. Our final objective was to determine the cost and price model of $1 \mathrm{~kW}$ and 5kW thermoelectric based Auxiliary Power Units (APU) on Class 8 trucks.

\subsection{Module Development and Fabrication}

\subsubsection{Skutterudite Leg Fabrication}

One of the main components in the development of a TEG is the fabrication of the thermoelectric legs used in the generator. The fabrication includes several different processes that are critical during the fabrication. These processes include synthesis of the skutterudite material, powder processing and hot pressing. Each process is described below.

\subsubsection{Skutterudite Synthesis}

The production of the synthesis of skutterudite material involves weighing high purity raw materials, sealing them in a quartz tube that is free of oxygen and then melting them in a furnace to obtain 100 to $200 \mathrm{~g}$ ingots. Our lab currently has 6 high temperature furnaces that allow us to make the P-type skutterudite material in 100-200 g batches to insure that we have good homogenous material. Our N-type skutterudite material has also been made in 100-200 gram batches and provides good robust material.

\subsubsection{Powder Processing}

After the $\mathrm{N}$ and P-type material is synthesized, the ingots are transferred into an inert gas glove box, where the material is powder processed. The powder processing of both the $\mathrm{N}$ and P-type skutterudite has been standardized for both materials. Each material is crushed and ground in a mechanical mortar and pestle and then milled using a planetary ball mill. During the ball milling the particle size is reduced by a factor of 2-3 times.

\subsubsection{Hot Pressing}

The hot pressing and metallization of the $\mathrm{N}$ and P-type material has been standardized for both materials. An optimum temperature and pressure during the hot pressing has produced strong and robust legs. Metalizing both sides in copper during the hot pressing procedure has improved the hot side bonding process. Over 40 pucks of both the $\mathrm{N}$ and P-type have been hot pressed to date. Our largest $50 \mathrm{~mm}$ die produces 56 legs that are diced into 5x7x7 mm leg (Figure 1.1). 


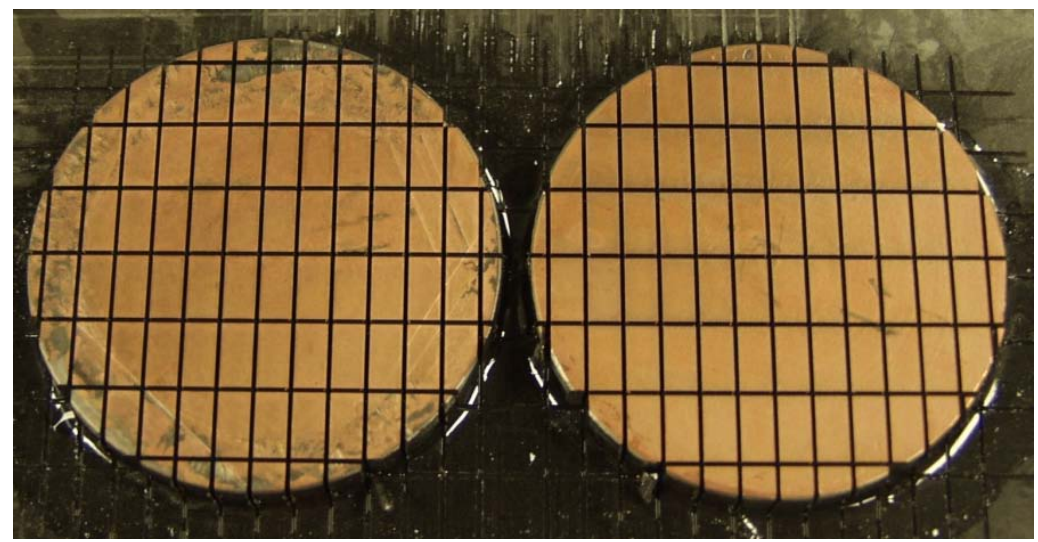

Figure 1.1. Hot pressed $\mathrm{N}$ and P-type metalized skutterudite pucks diced into 3.5x7x7mm legs.

\subsubsection{Couple Fabrication}

The high-temperature couples used in this experiment were constructed by joining two 3.5x7x7mm skutterudite legs (one N-type and one P-type) with a copper heat exchanger, or hot shoe/contact (Figure 1.2). Applying a temperature gradient between the copper hot shoe and the bottom of the legs causes a current flow and voltage potential throughout the material. With a temperature gradient of approximately $500^{\circ} \mathrm{C}$ each component will produce approximately $1 \mathrm{~W}$ of power under normal testing conditions.

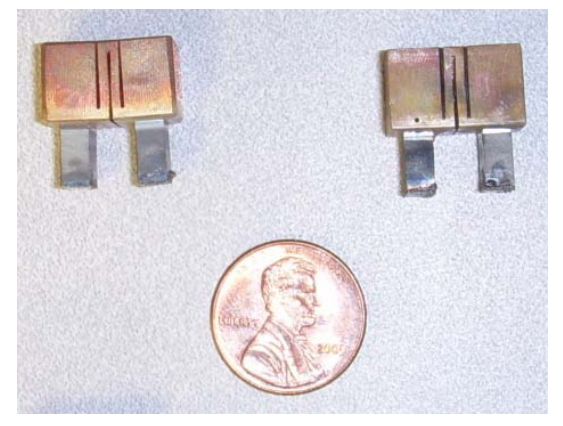

Figure 1.2. A skutterudite $\mathrm{N}$ and P-type couple bonded to a copper hot shoe/contact.

\subsubsection{Module Fabrication}

After a batch of skutterudite couples has been fabricated, the couples are then used to fabricate modules. A 10 leg module (5 N and P-type couples) is fabricated by soldering 5 couples to tin coated copper plates (cold shoes) in series. A copper plate with a brass fastener was soldered onto each end of the module to allow it to be bolted to a generator box (Figure 1.3). The modules contained five $1 \mathrm{~W}$ couples connected in series; each module was capable of producing around 5W under normal testing conditions. 


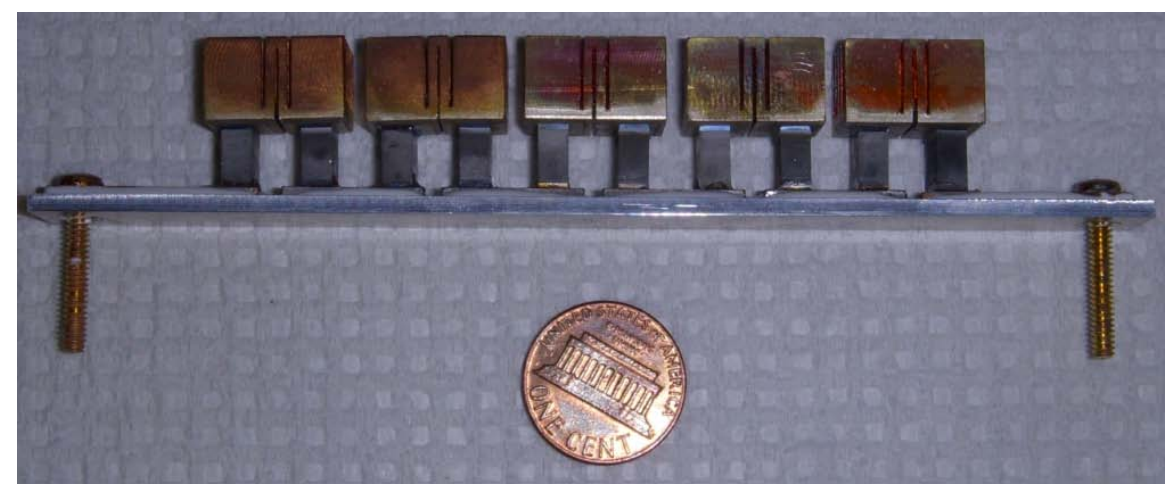

Figure 1.3. A 10 leg module capable of producing $5 \mathrm{~W}$.

\section{TEG System Design}

Testing of the modules was divided into 3 systems: heating, cooling, and electrical (shown in Figure 2.1). The heating system starts with nitrogen gas as the working fluid. The nitrogen starts as a compressed gas and is regulated into a heating airtorch system. The nitrogen gas first flows though a Meriam Instruments laminar flow element to measure the flow rate and then into the airtorch. A flow divider is then used to force the nitrogen gas through flow tubes directed onto the TEG. After heating the TEG, the gas is diluted with cold air and exhausted. Temperatures are measured at every stage within the system including before the heater in the TEG and after the TEG.

The cooling system starts with tap water that flows through four Gems Sensors Roto flow meters to measure the flow rate and then through copper cold plates where the modules are in contact to remove heat. The water temperature is measured before the entering the cold plate and after exiting to find the $\Delta \mathrm{T}$ and thus, the energy gained.

The electrical system consists of the TEG Kepco Power Supply used to backload the modules and an Omega OMB-DAQ-56 data acquisition system to monitor and record the voltages of each TEG module.

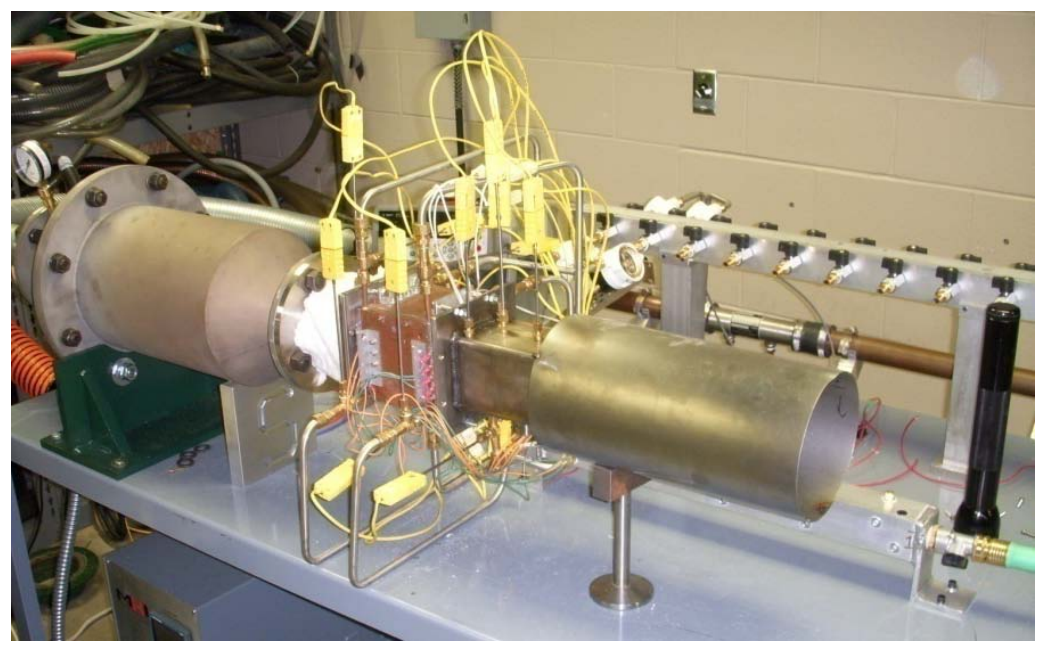

Figure 2.1. System consisting of a heater, cooling system and electrical components used for testing a TEG. 
In our initial design of the TEG, we tested multiple couple designs and TEG flow configurations to produce optimum output from the thermoelectric material. We concluded that our hot shoe design needed to capture more heat. The hot shoe was redesigned from a flat hot shoe to a fin type hot shoe that had more than 20 times the heat sink area (Figure 2.2). We also made the base design of the 10 leg modules more robust by mounting them on a base plate which could be easily bolted in and out of the TEG.
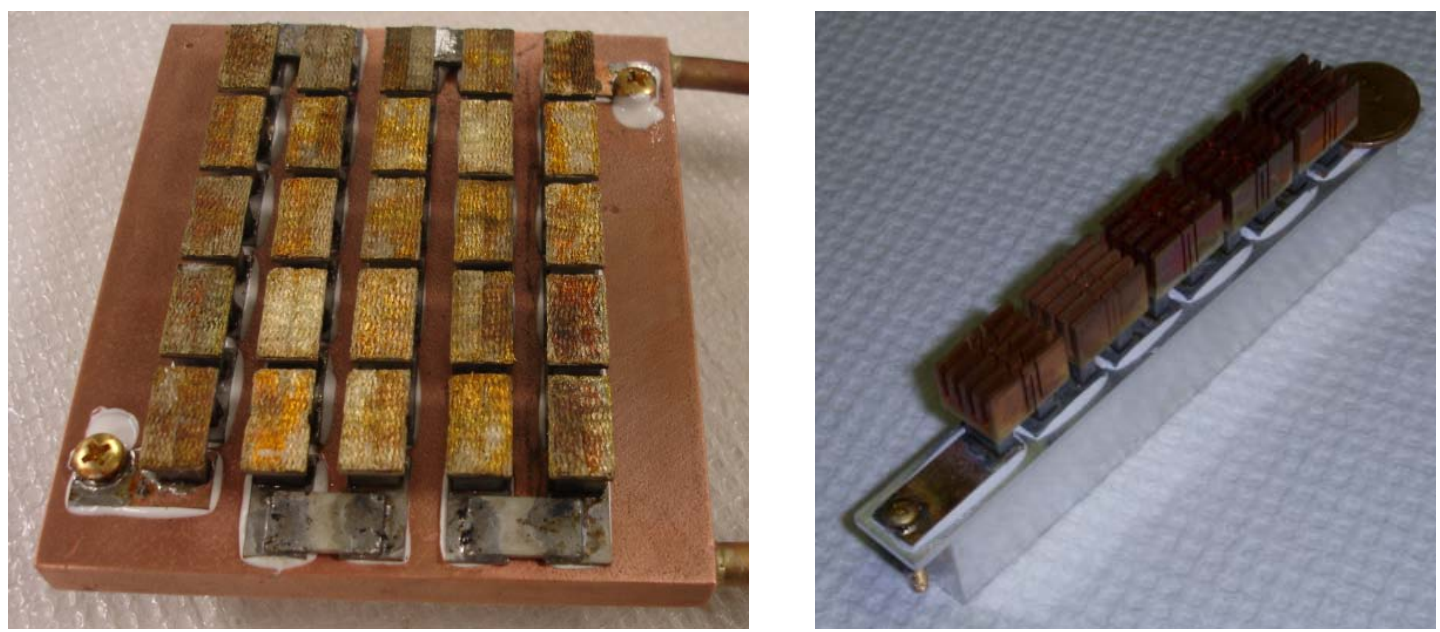

Figure 2.2. TEG with flat hot shoe design compared to a 10 leg module with heat sink hot shoe.

\subsection{W TEG}

The first 10 leg module with heat sink hot shoes was tested using tubes to direct the flow of the nitrogen gas onto the top of the module. As shown in Figure 2.3, an infrared camera was used to observe the heating distribution of the module.

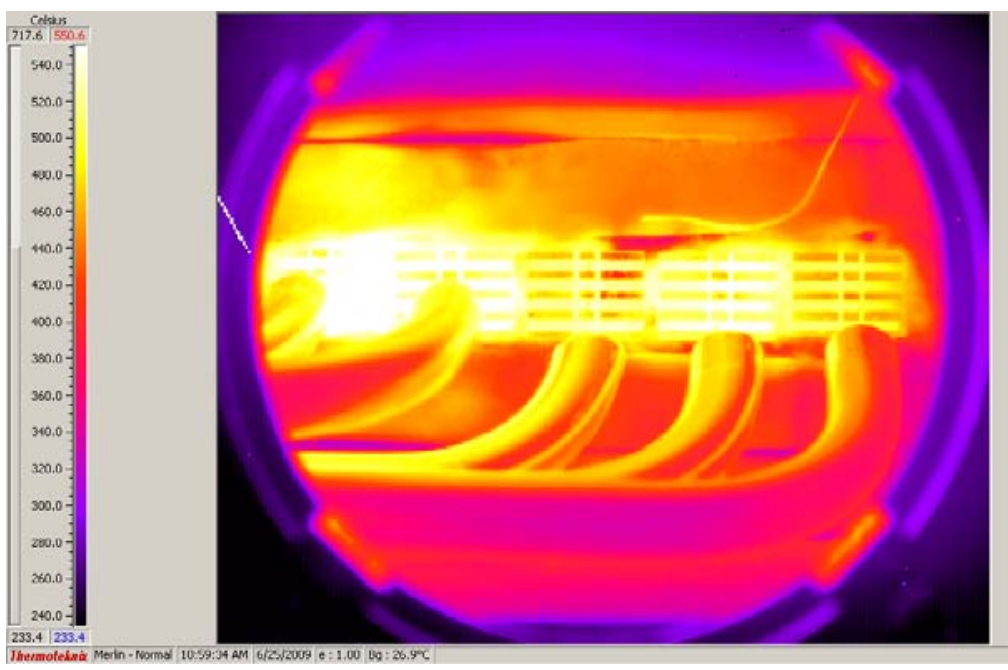

Figure 2.3. Infrared picture of a 10 leg module within the TEG. 
The camera allowed us to estimate the temperature of the each of the hot shoes and determine some of the gas flow characteristics that affect the modules performance. This setup was tested 2 different times to demonstrate repeatable power outputs for the module. The nitrogen gas was heated to $685^{\circ} \mathrm{C}$ with a flow rate of $9.7 \mathrm{CFM}$. The hot shoes reached a temperature of $500-550^{\circ} \mathrm{C}$. In both tests, the module produced $5.4 \mathrm{~W}$ and an open circuit voltage of .725 volts. Both of the power and voltage curves are shown in Figure 2.4.

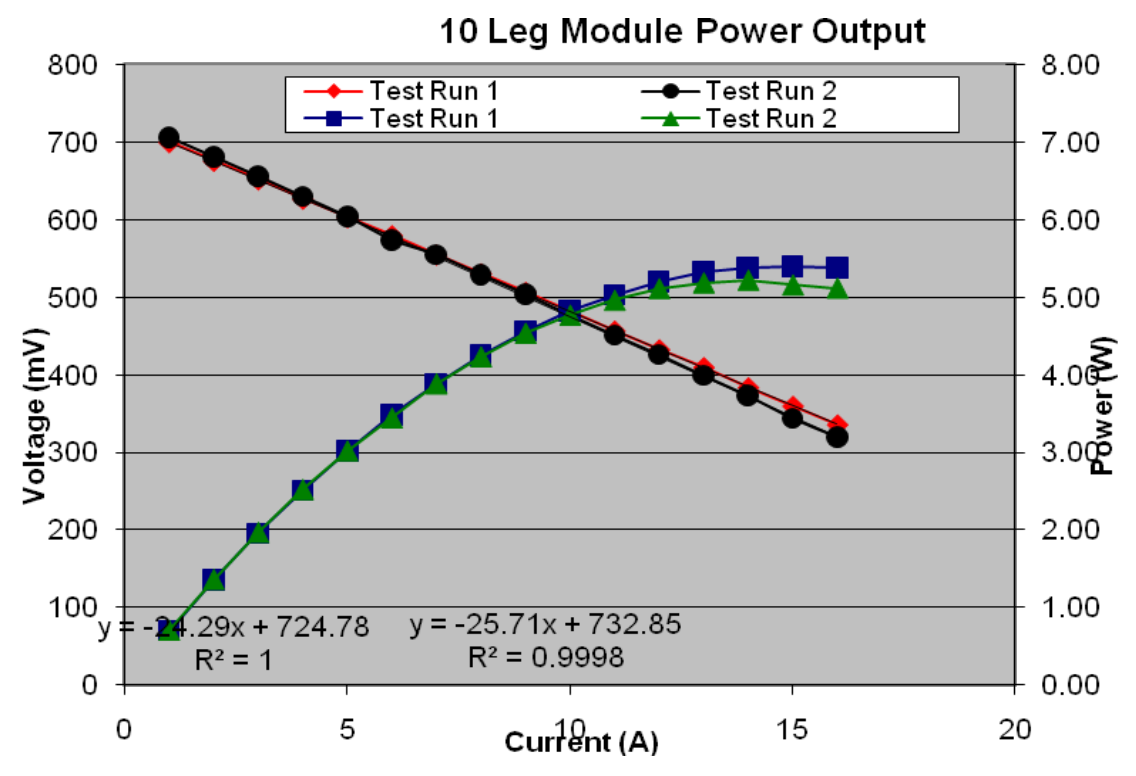

Figure 2.4. Voltage and power output curves for a single 10 leg module for 2 different test runs.

\subsection{W TEG}

Based on results of the 5 W TEG a 25 W TEG was fabricated using 5- 10 leg modules. The modules were placed horizontally on one of the sides of the TEG's cooling plate box. A tube bank was then inserted to direct gas flow on top of each module (Figure 2.5). Using this configuration, the inlet gas was 710 degrees $\mathrm{C}$ at $14 \mathrm{CFM}$. The hot shoe temperature was $610^{\circ} \mathrm{C}$ and the cold side was $115^{\circ} \mathrm{C}$, giving a $500^{\circ}$ temperature difference. The maximum output for the highest power producing module was $6.4 \mathrm{~W}$ and the lowest was $3.96 \mathrm{~W}$. The power output data for all 5 modules along with the open circuit voltages can be seen in Table 2.1. As shown in Figure 2.6, the average output of the 5 modules was $4.9 \mathrm{~W}$ and they produced a total power output of 24.6 W. The voltages and power outputs from each module were highest in the center and decreased toward the outside. We believe that this is associated with cooling within the TEG; modules 1 and 5 are closer to the top and bottom cooling plates making them produce slightly less voltage/power. Module 1 , which produced .67 volts and $4.32 \mathrm{~W}$ of power, was the same module in the first $5 \mathrm{~W}$ TEG test. 

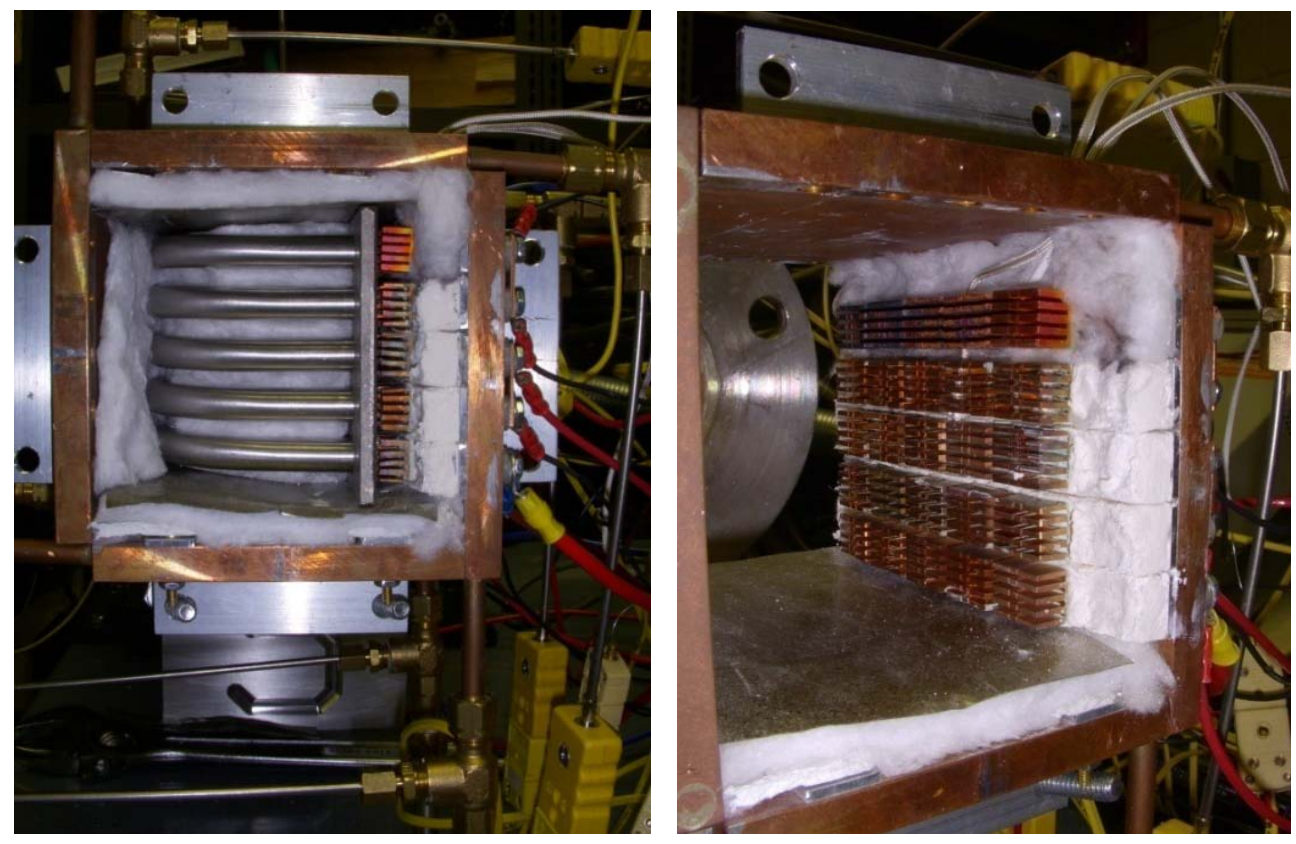

Figure 2.5. Test setup of $25 \mathrm{~W}$ TEG with and without flow tubes.

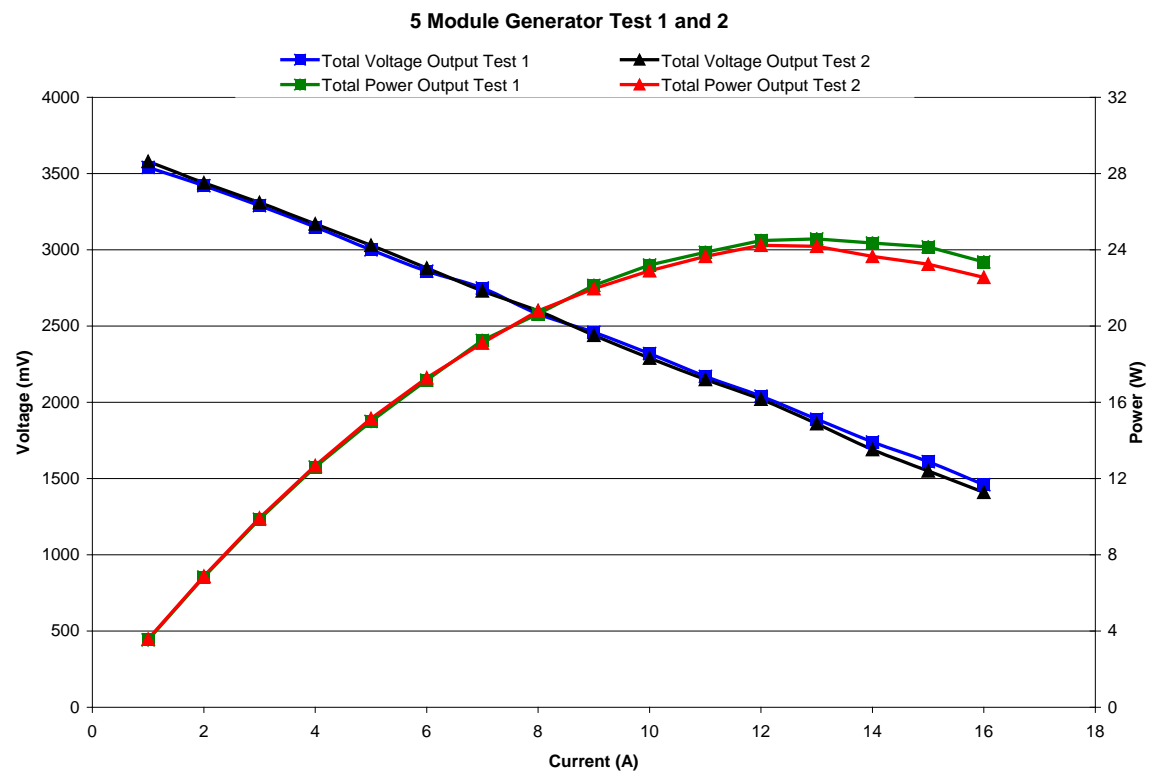

Figure 2.6. Voltage and power output curves for a five-10 leg modules for 2 different test runs. 
Table 2.1. Voltage and power output from each module in the $25 \mathrm{~W}$ TEG.

\begin{tabular}{|c|c|c|}
\hline Module & $\begin{array}{c}\text { Max open circuit voltage } \\
\text { at temperature (mVolts) }\end{array}$ & $\begin{array}{c}\text { Max power at } \mathbf{\Delta} \mathbf{T}=\mathbf{5 0 0}^{\mathbf{0}} \mathbf{C} \\
\text { (amps) }\end{array}$ \\
\hline 1 & 670 & 4.32 \\
\hline 2 & 730 & 4.68 \\
\hline 3 & 790 & 6.4 \\
\hline 4 & 720 & 5.2 \\
\hline 5 & 670 & 3.96 \\
\hline
\end{tabular}

\section{$2.350 \mathrm{~W}$ and 100W TEG}

Many different aspects were incorporated into the design of the TEG to achieve the desired performance. Some of these aspects include ease of manufacturing, modularity, and expandability. The main portion of the generator was constructed with four copper cooling plates orientated into a box. These four plates were cooled with water and each accommodated five TEG modules. For the 50W test, only two plates had modules; with the $100 \mathrm{~W}$ test, all four plates had modules. The flow rate and the inlet and outlet temperatures were monitored.

A $12 \mathrm{KW}$ electric air torch was used to heat nitrogen gas which was then blown onto each TEG module using a flow divider. The flow divider consists of a box with one open end and one closed end. Holes were drilled on the sides of the box and were positioned directly over each module component. For the 50W test, a flow divider was made with 50 holes; 25 holes on 2 sides. For the $100 \mathrm{~W}$ test, another divider was made with 100 holes; 25 holes on each of the 4 sides (Figure 2.7).

An important design consideration ensured equal air flow through each hole in the air distributer; equal air flow through the holes is necessary to ensure even heating of the module's hot shoes. To determine the maximum hole diameter, the following fluids equations were used:

$$
\Delta \mathrm{P}=\frac{1}{2} \frac{\rho}{2} \mathrm{Vel}^{2}
$$

Equation 2-1

$$
\dot{V}=n \frac{D^{2}}{4} \pi * V e l
$$

Equation 2-2

The hole size in the divider for the 100W test was designed the keep a pressure drop across the divider of 5 psi and a hole velocity of approximately $19.7 \mathrm{~m} / \mathrm{s}$. For both air distributors, the inflow rate of nitrogen was assumed to be 14 CFM. 


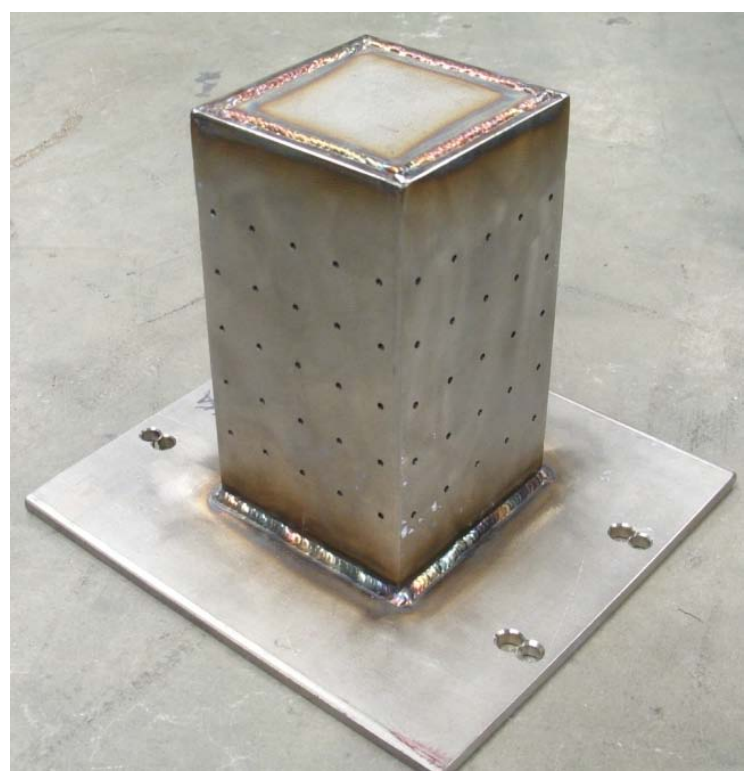

Figure 2.7. Nitrogen gas flow divider for $100 \mathrm{~W}$ test.

To verify equal flow air the velocity was measured from each of the holes on one side of the air distributor using a Dwyer No. 400 manometer, while pumping 14 CFM of nitrogen gas through the generator box. Because all of the holes had equal diameters, and therefore equal area, we used the following relation to verify equal flow rates for each of the holes:

$$
\dot{V}=A * V e l
$$

Equation 2-3

\section{Generator Testing}

\subsection{Test Process}

The test process for the generator was as follows. The nitrogen gas and the water were turned on along with the heater. Once the heater reached its maximum output the modules were tested. Using the Omega data acquisition system, data was continually collected throughout the entire experiment. Measurements were taken every 2.5 seconds and recorded in Microsoft Excel. The flow rates of both the water and the gas were measured along with the temperatures of the water before and after the cooling plates and the temperature of the nitrogen before and after the heater in the generator box and after the generator box. The temperatures of the hot and cold shoes were also measured.

To achieve the large temperature gradient needed to test the TEG the heater nodes were heated to $925^{\circ} \mathrm{C}$ for over a period of 20 minutes. During this time nitrogen passed through the system at a rate of approximately $14 \mathrm{CFM}$. Once the nodes reached $925^{\circ} \mathrm{C}$ the nitrogen flow rate was increased to approximately 14-15 CFM (the highest flow rate at which the heater could still maintain a node temperature of $925^{\circ} \mathrm{C}$ ). This created the temperature gradient needed to test the TEG. At the same time the water flowed at a rate of approximately 1.5 gallons per minute and a temperature rise of roughly $6.5^{\circ} \mathrm{C}$ from 21 to 27.5 . 
After the modules were heated to the maximum temperatures that could be achieved with this setup the power was then tested. This was done by driving 16 incrementing amperages across each module individually and then recording the voltage measured across the module at that amperage. Using the equation $\mathrm{P}=\mathrm{VI}$ we calculated a power curve for each of the modules (shown in Figure 3.1). After each module had been individually tested the heater was ramped back down to room temperature over a period of 10 minutes.

This test was performed four times with the 50W generator and once with the 100W generator. After each test the modules that performed below expectations and had high resistivity slopes due to a mechanical break in the skutterudite were replaced with new modules.

\subsection{Results}

Figure 3.1 shows that all of the modules seem to have similar resistivity slopes and they produced their maximum power of between 12 to 15 amps. Table 3.1 shows the maximum $\Delta \mathrm{T}$, the peak generator power, (both at constant amperage and at varying amperage) and the number of modules that performed below expectations for each of the five runs. For the fourth run of the $50 \mathrm{~W}$ generator (taking average degradation values into account) extrapolated data was used for the 3 modules which performed below expectations. The total voltage and power output from the $4^{\text {th }}$ run is shown in Figure 3.2.

Table 3.1. Power output from 50W and 100W TEG.

$\begin{array}{ccccc}\text { Run \# } & \text { Max } \Delta \mathrm{T} & \begin{array}{c}\text { Peak Power } \\ \text { (Same Amp) }\end{array} & \begin{array}{c}\text { Peak Power } \\ \text { (Diff Amp) }\end{array} & \begin{array}{c}\text { \# Performed Below } \\ \text { Expectations }\end{array} \\ 50-1 & 534^{\circ} \mathrm{C} & 42.5 \mathrm{~W} & 45.3 \mathrm{~W} & 2 \\ 50-2 & 545^{\circ} \mathrm{C} & 43.8 \mathrm{~W} & 48.2 \mathrm{~W} & 2 \\ 50-3 & 552^{\circ} \mathrm{C} & 48.0 \mathrm{~W} & 50.2 \mathrm{~W} & 1 \\ 50-4 & 550^{\circ} \mathrm{C} & 50.1 \mathrm{~W}^{*} & 50.4 \mathrm{~W}^{*} & 3 \\ 100-1 & 440^{\circ} \mathrm{C} * & 70.4 \mathrm{~W} & 74.7 \mathrm{~W} & 6 \\ & * \text { Extrapolated } & & & \end{array}$




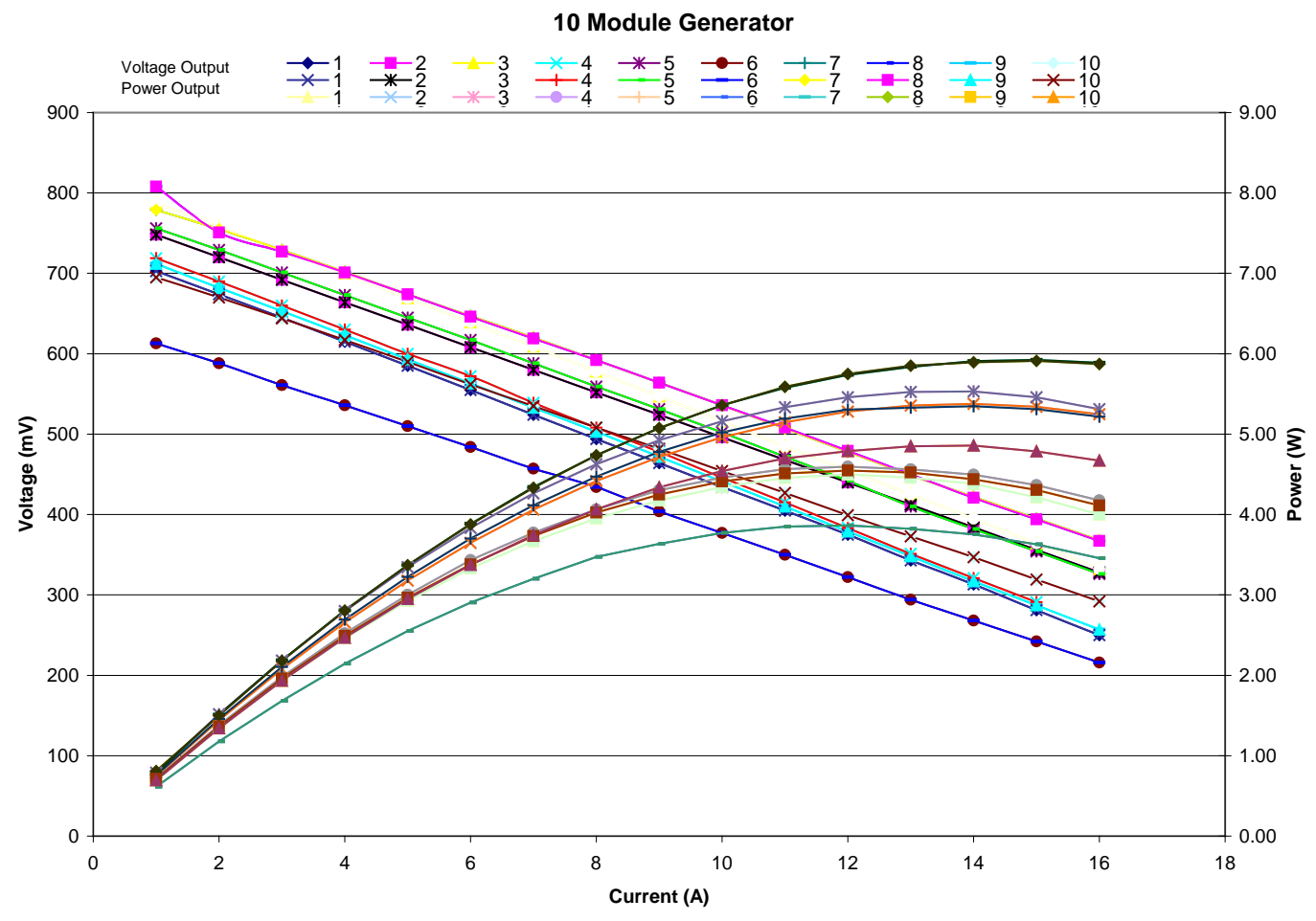

Figure 3.1. Power output curves for run 50-4.

\section{Module Generator Power Output}

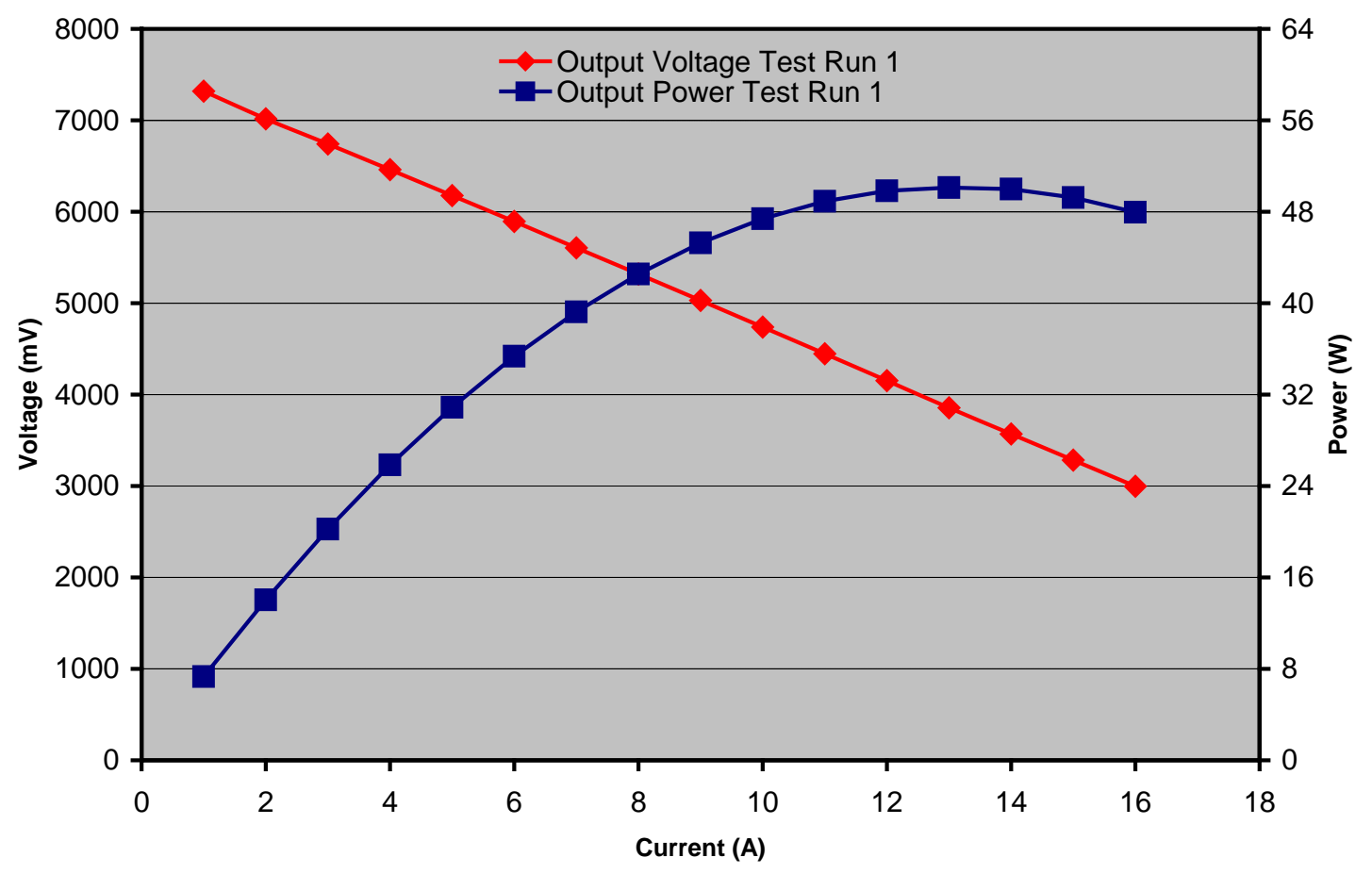

Figure 3.2. Total voltage and power output curves for a 10 module TEG. 


\section{Heat Balance}

The first law of thermodynamics states that, "energy can neither be created nor destroyed; it can only change forms" [6] Thus, we know that the electrical energy generated by the TEG is really converted heat energy from the nitrogen gas.

At steady state, the heated nitrogen gas gained approximately $7.5 \mathrm{~kW}$ of heat as it passed from the intake through the heater during the $50 \mathrm{~W}$ tests. The gas lost approximately $2 \mathrm{~kW}$ of heat as it passed through the generator box and it thus exited with a net of $5.5 \mathrm{~kW}$ of extra heat (Figure 4.1). For the $100 \mathrm{~W}$ test, the nitrogen gained approximately $8 \mathrm{~kW}$ of heat from the intake through the heater, lost approximately $2.5 \mathrm{~kW}$ in the generator box and exited with a net of approximately $5.5 \mathrm{~kW}$ of extra heat. To calculate this heat loss, the following equation was used [7]:

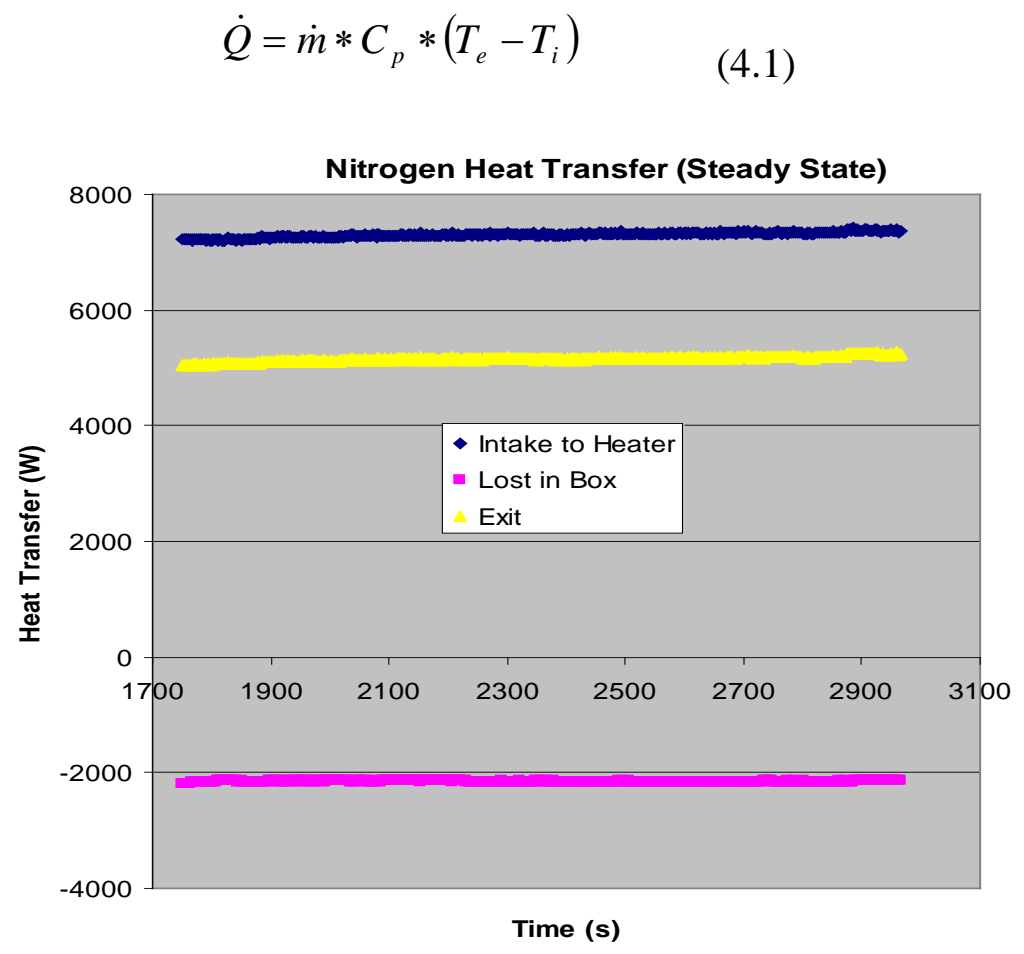

Figure 4.1. Nitrogen heat transfer for run 50-3.

With the generator box as our system, the total $\dot{Q}$ should be 0 . Thus, all of the heat lost by the nitrogen gas should be accounted for with the heat gained by the water and the electrical energy generated by the TEGs.

To calculate the heat transfer of the nitrogen gas, the following equation was derived to calculate the specific heat of the nitrogen at any temperature between $-98^{\circ} \mathrm{C}$ and $1227^{\circ} \mathrm{C}$ :

$$
C_{p}(\text { Temp })=-2 * 10^{-10} * \text { Temp }^{2}+3 * 10^{-7} * \text { Temp }+3 * 10^{-5}+1.0379
$$


This was determined by plotting the known specific heat values of nitrogen from $-98^{\circ} \mathrm{C}$ to $1227^{\circ} \mathrm{C}$ and then calculating the function of the corresponding trend line [8]. The above function fits the known specific heat values with an $\mathrm{R}$ value of 0.9999 . Because the temperature of the water only ranged from approximately $18^{\circ} \mathrm{C}$ to approximately $31^{\circ} \mathrm{C}$, the specific heat of the water was assumed to be a constant $4.182\left(\mathrm{~kJ} / \mathrm{kg}^{*} \mathrm{~K}\right)[9]$.

The above equations were used to determine the rate of heat loss of the nitrogen gas as it passed through the generator box. The rate of heat gain of the water was also calculated. The modules were assumed to consume 50W of heat during steady state. For this system, we established the following relation:

$\dot{Q}_{\text {Nitrogen }}=\dot{Q}_{\text {Water }}+\dot{Q}_{T E G s}+\dot{Q}_{\text {Unaccounted }}$

The values of $\dot{Q}_{\text {Nitrogen }}, \dot{Q}_{\text {Water }}$ and $\dot{Q}_{\text {TEGs }}$ were plotted with respect to time, Figure 4.2. Using these plots, we calculated the average $\dot{Q}_{\text {Unaccounted }}$ for the portion of the experiment that measured the power output of the modules. We then plotted the absolute value of $\dot{Q}_{\text {Unaccounted }}$ as a percentage of $\dot{Q}_{\text {Nitrogen }}$ and found that approximately $3 \%$ of the total heat lost by the nitrogen was unaccounted for during the steady state testing portion of the experiment (Figure 4.2).

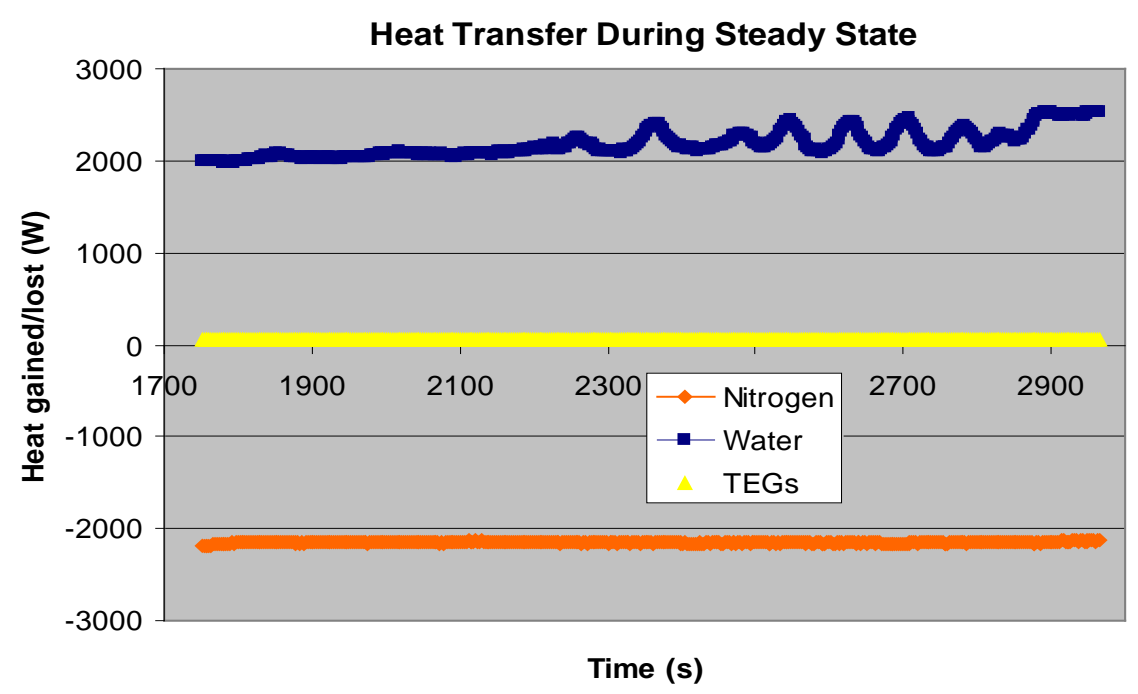

Figure 4.2. Steady state heat transfer for run 50-3. 


\section{Heat From Nitrogen (W)}

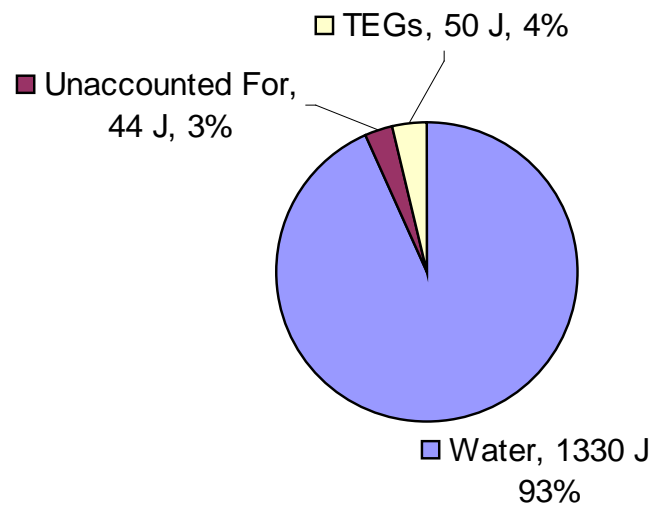

Figure 4.3. Heat distribution for run 50-2.

For the $50 \mathrm{~W}$ generator tests, approximately $60 \%$ of the heat that the water gained during the testing portion of the experiments was transferred across the two plates that held the bolted TEG. This means that approximately $40 \%$ of the heat from the nitrogen never came in contact with the TEG. Thus, only approximately $60 \%$ of the total amount of heat lost by the nitrogen, while in the box, was used to calculate the efficiency of the TEG. Table 4.1 shows the TEG efficiency results of the tests.

Table 4.1. TEG Efficiencies

$\begin{array}{cc}\text { Run \# } & \text { TEG Efficiency } \\ 50-1 & 3.1 \% \\ 50-2 & 3.7 \% \\ 50-3 & 3.8 \% \\ 50-4 & 4.4 \% \\ 100-1 & 3.8 \%\end{array}$

\subsection{TEG System Temperatures}

Nine thermocouples were used in the $50 \mathrm{~W}$ tests to measure the nitrogen temperature directly after leaving the heater and inside the generator box. Inside the box, eight thermocouples were used to measure the temperature of the hot and cold shoes, the stream wall temperature, and a five-layer temperature profile, Figure 4.4. Table 4.2 shows the various temperature results with the heater maxed out and the flow rate of 14-15 CFM at a steady state for each of the tests. 


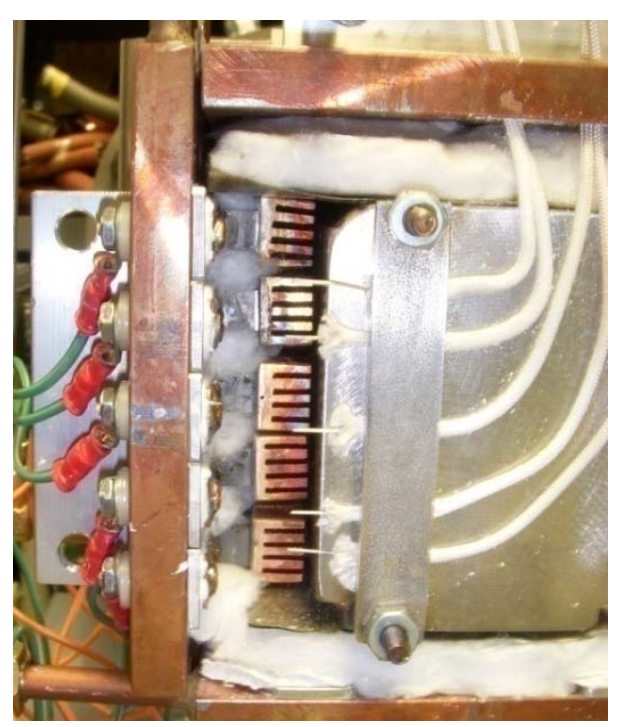

Figure 4.4. Five-layer thermocouple profile for 50W generator.

Table 4.2. TEG temperature results.

$\begin{array}{ccccc}\text { Run \# } & \text { Inlet Max } & \text { Hot Shoe Max } & \begin{array}{c}\text { Cold Shoe } \\ \text { Max }\end{array} & \text { Stream Wall Max } \\ 50-1 & 706^{\circ} \mathrm{C} & 625^{\circ} \mathrm{C} & 100^{\circ} \mathrm{C} & 670^{\circ} \mathrm{C} \\ 50-2 & 722^{\circ} \mathrm{C} & 650^{\circ} \mathrm{C} & 105^{\circ} \mathrm{C} & 670^{\circ} \mathrm{C} \\ 50-3 & 723^{\circ} \mathrm{C} & 655^{\circ} \mathrm{C} & 100^{\circ} \mathrm{C} & 670^{\circ} \mathrm{C} \\ 50-4 & 716^{\circ} \mathrm{C} & 650^{\circ} \mathrm{C} & 105^{\circ} \mathrm{C} & 670^{\circ} \mathrm{C} \\ 100-1 & 790^{\circ} \mathrm{C} & 565^{\circ} \mathrm{C} & 125^{\circ} \mathrm{C}^{*} & 585^{\circ} \mathrm{C} \\ & * \text { Extrapolated } & & & \end{array}$

\subsection{TEG Exit Temperatures}

In order to correctly calculate the total amount of heat transfer that occurs in the generator during the test, it is necessary to take accurate temperature measurements of the nitrogen gas both at the entrance of the generator and at the exit. The temperature difference between the entrance and exit temperatures of the nitrogen is directly proportional to the heat transfer of the nitrogen.

To ensure certainty of the nitrogen temperature measurements, a four-thermocouple grid was constructed to measure the exit temperature of the nitrogen for the 50W test. Over the four runs with the $50 \mathrm{~W}$ generator, the measurements for the exit temperature of the nitrogen varied by up to more than $100^{\circ} \mathrm{C}$, Figure 4.5. To further explore this discrepancy, a ten-thermocouple grid was constructed to measure the exit temperature of the nitrogen for the 100W test, Figure 4.6. In addition, a six-thermocouple grid was constructed to measure the inlet temperature of the nitrogen to see if a similar discrepancy existed at the inlet, Figure 4.7. 


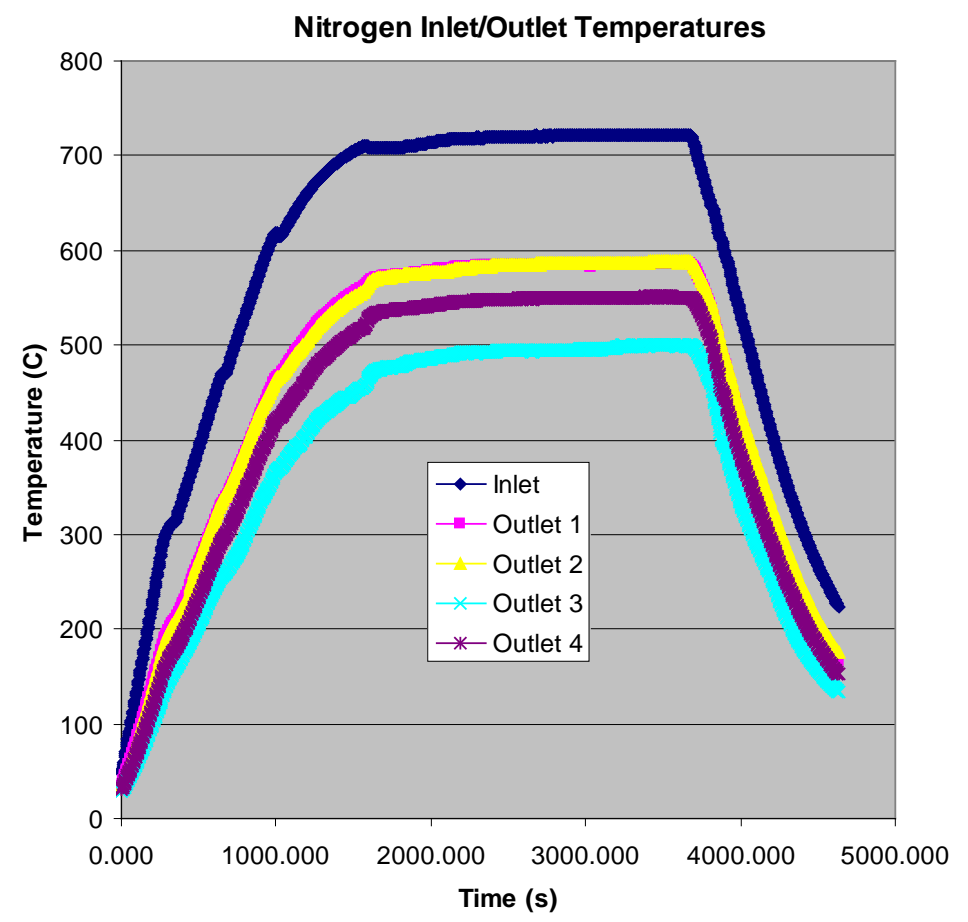

Figure 4.5. Nitrogen inlet and outlet temperatures for run 50-2.

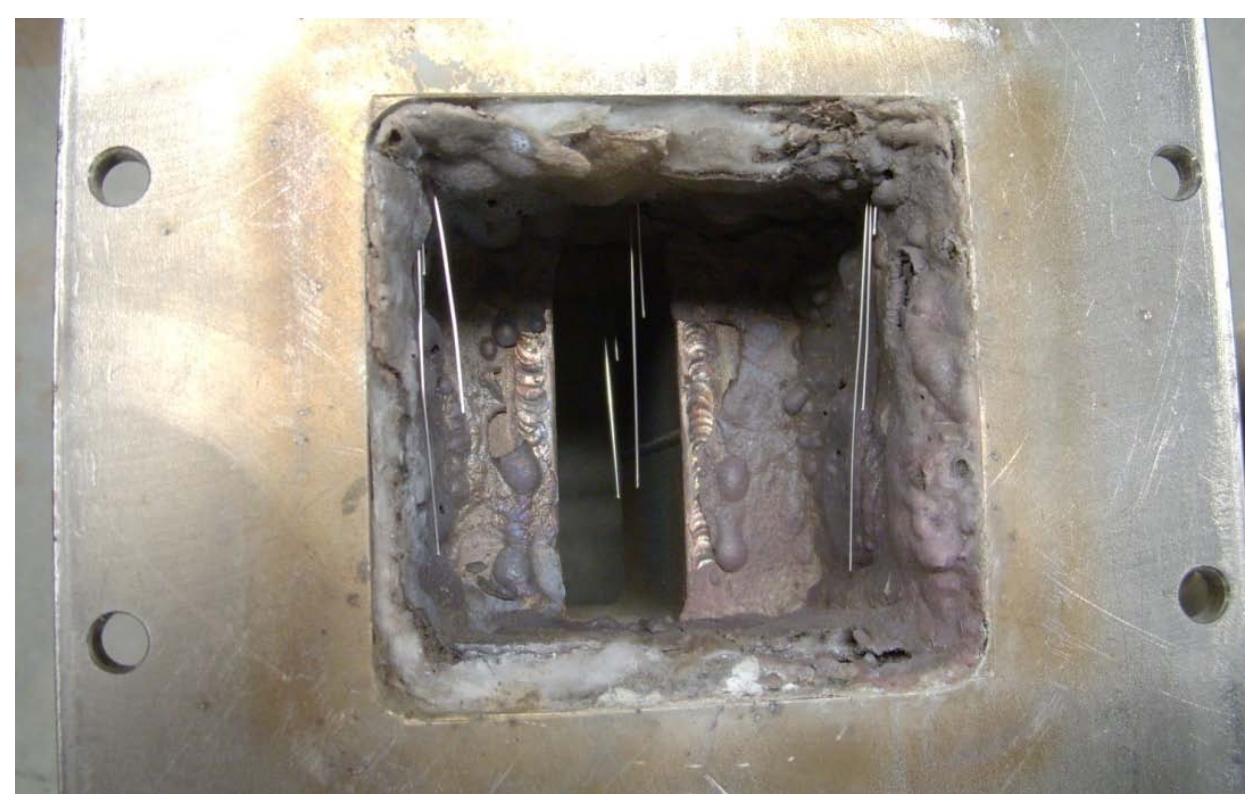

Figure 4.6. Nitrogen exit thermocouple grid for run 100-1. 


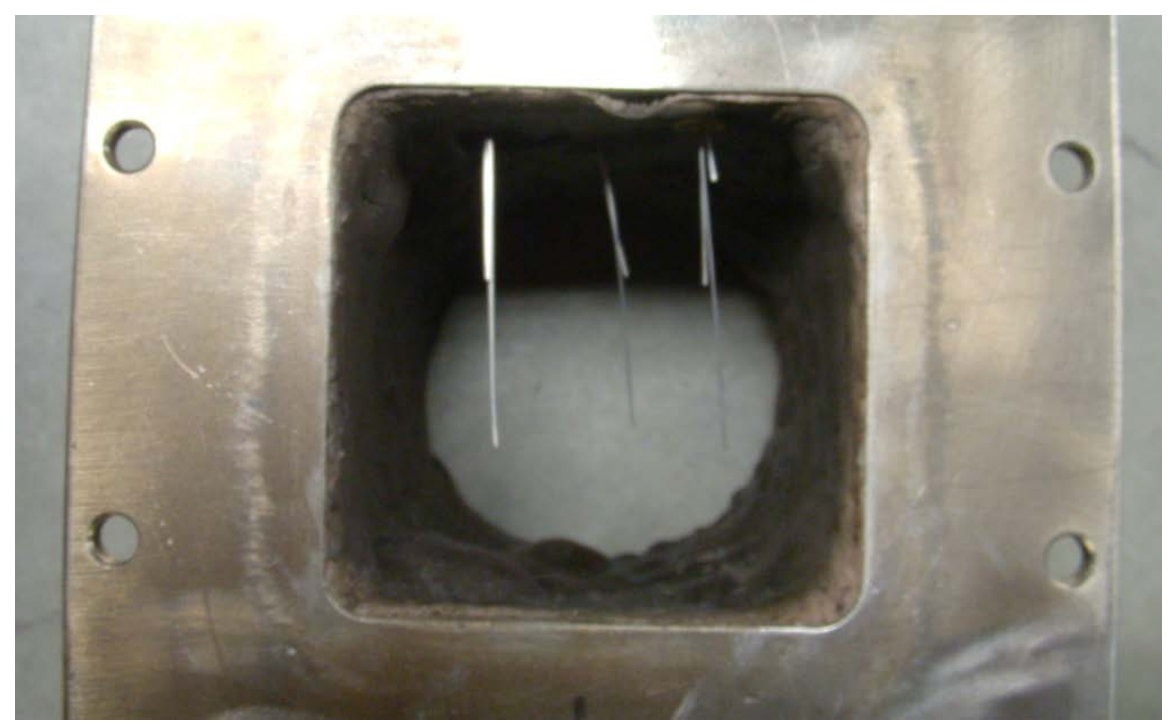

Figure 4.7. Nitrogen inlet thermocouple grid for run 100-1.

Figure 4.8 shows that the measured inlet temperatures of the nitrogen gas were all within a few degrees of each other for the entire duration of the test. Figure 4.9 shows that the measured exit temperatures varied by up to more than $150^{\circ} \mathrm{C}$.

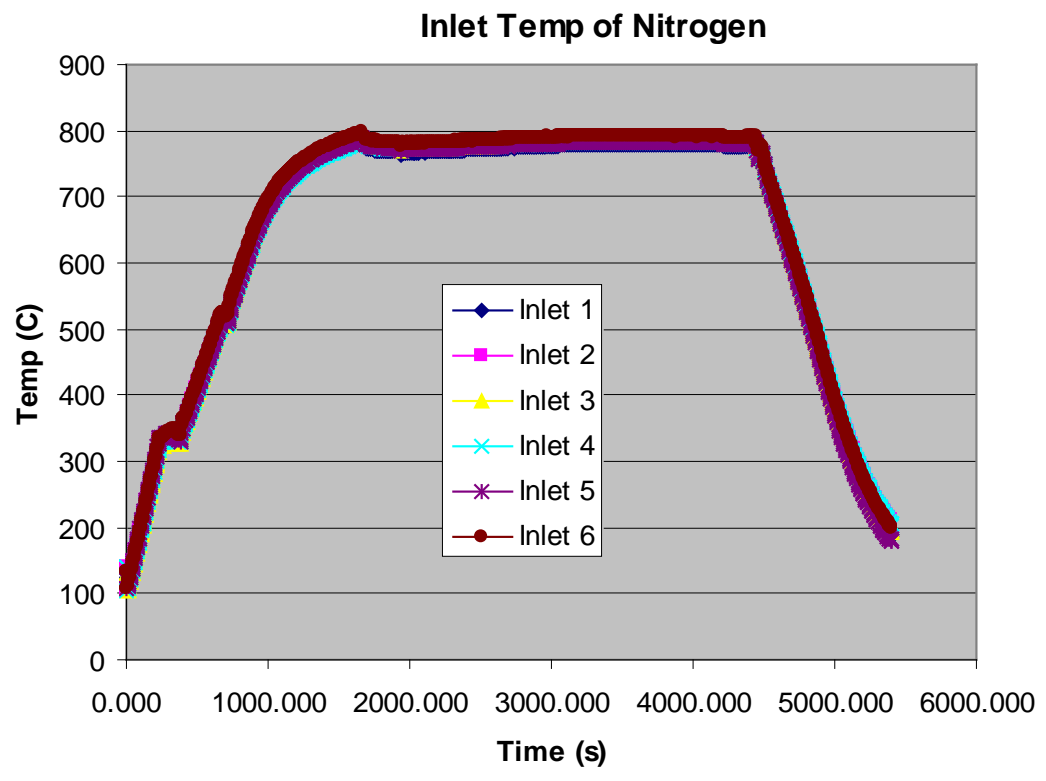

Figure 4.8. Nitrogen inlet temperature for run 100-1. 


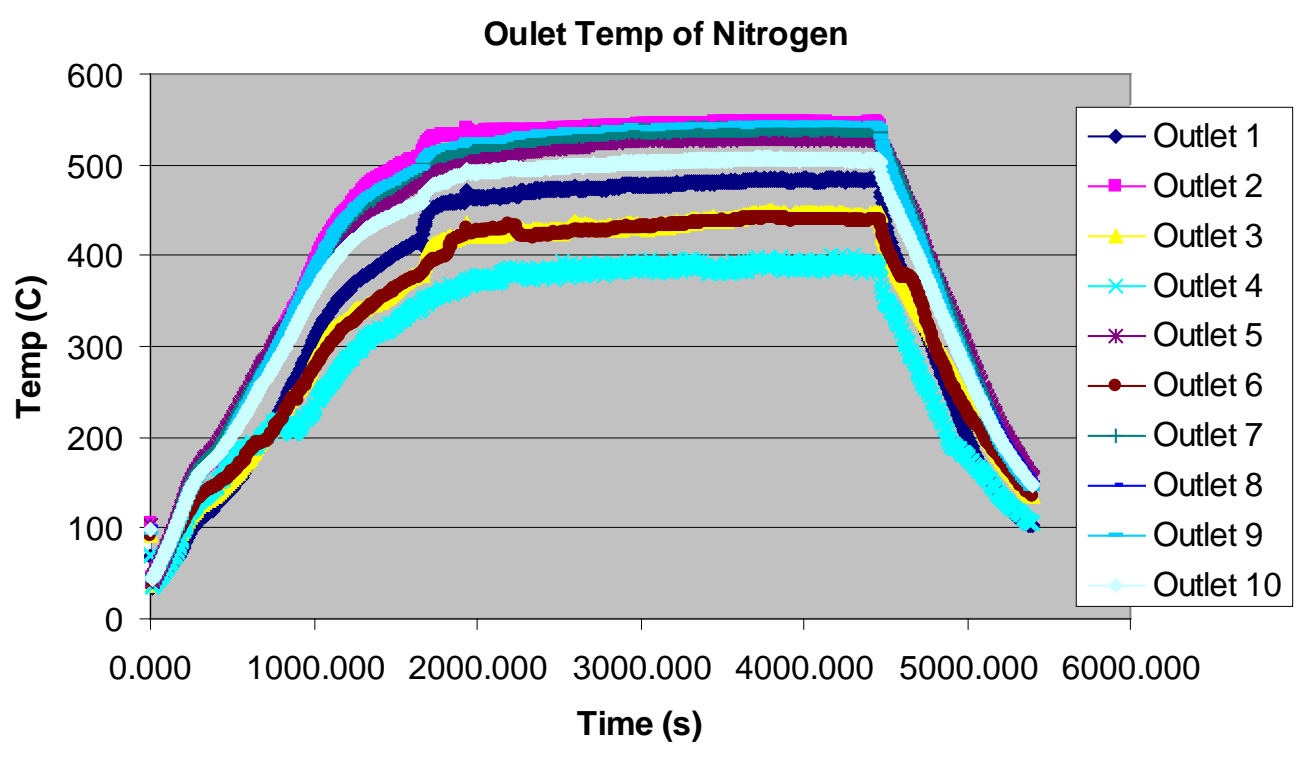

Figure 4.9. Nitrogen exit thermocouple grid for run 100-1.

Some of these variations were caused by thermocouple placement. The flow geometry coming through the exhaust is extremely hard to predict, thus the experiment was instrumented with more than just one thermocouple to try to examine that geometry. We found that there were differences in temperatures for each thermocouple and concluded that some of the lower temperature thermocouples were placed too close to the wall and were out of the flow path or were in a dead/hot-cold mixing area that was closer to the initial exhaust exit from the generator. The thermocouples that were placed farther downstream had better readings because the gas had sufficient time to mix. However, this also allowed the gas to cool slightly, making an accurate reading hard to obtain.

\subsection{TEG Durability}

As we repeated this test several times, two issues became very apparent:

1. The modules produced slightly lower voltages for the same amperages with each iteration. This resulted in slightly lower power values for each module every time the test was repeated. This degradation is likely caused by fissures and micro-cracking that occurs in the skutterudite material due to exposure to the high temperatures of these tests. Other possibilities for this degradation include sublimation and material phase change.

2. At least once with every test a module degraded to the point that it no longer produced a significant voltage at higher amperages. This was caused by an accumulation of fissures and micro-cracking that results in a complete break in the skutterudite leg. Whereas the previous degradation was gradual (losing approximately $0.2 \mathrm{~W}$ every run), this degradation was rather abrupt and usually resulted in a maximum power of less than $2 \mathrm{~W}$ for that module.

These two issues raise very serious concerns about the life expectancy of the TEG. In order for the TEG to be cost-effective, improvements must be made to greatly reduce the gradual and the abrupt degradation of the module's power output. To determine what kinds of improvements 
must be made these power reductions must be investigated to determine if the degradation is due to an issue with the skutterudite material or with the module design.

To achieve our goal of designing, fabricating and demonstrating a 1-KW thermoelectric generator, a close look must be taken of the skutterudite material and the fabrication process of the module legs. Improvements to the material/fabrication process must be made to allow the modules to undergo many cycles of high-temperature tests without failing. Module durability and robustness must also be improved to make the TEG feasible for real-life applications. In addition, a new module design must be developed to feature lower resistivity values.

\subsection{TEG Conclusions}

A 50W generator was designed and fabricated to test the individual voltage drop of 10 TEG modules when exposed to 16 incrementing driving amperages, (from 1 to 16 amps) at a temperature gradient of approximately $550^{\circ} \mathrm{C}$. Heated nitrogen gas was used to heat the hot shoe, and water was used to cool the cold shoe to achieve this temperature gradient. The nitrogen gas gained approximately $7.5-8 \mathrm{~kW}$ of heat from the intake through the heater and lost approximately 2-2.5 kW in the generator box and thus exited with a net of approximately $5.5 \mathrm{~kW}$ of extra heat. This test was performed four times, replacing TEG modules that performed lowerthan-expected with new TEG modules after each test. Extrapolated values from previous tests (taking degradation into account) were used for the modules that performed lower than expected during the fourth run. For this run, the combined power output of the 10 modules was approximately $50.1 \mathrm{~W}$ at a steady 13 amps. The sum of the maximum power achieved for each of the 10 modules was approximately $50.4 \mathrm{~W}$. A heat transfer analysis confirmed that at least $96 \%$ of the heat released by the nitrogen gas was absorbed by either the cooling plates or the TEG during the testing portion of the experiment. The efficiency of the TEG was approximately $4 \%$.

\section{TEG Insulation}

\subsection{Aerogel Activities}

Three thrusts comprised the aerogel-insulation activities. First, the first generation aerogel formulation was integrated into multiple couples, subassemblies, individual panels and one entire TEG (Figure 5.1). The formulation was first developed at JPL and has been modified by the Sakamoto group at MSU such that it is compatible with the Skutterudite TEG technology. Secondly, efforts to improve and characterize the next generation aerogel were conducted. Attempts to improve the consistency from batch to batch were conducted and a preliminary test to measure the thermal insulation efficacy was completed. Lastly, efforts to significantly reduce the cost of aerogel encapsulation were initiated. The key to reducing costs will likely require the ability to dry the aerogel under ambient conditions rather than in an autoclave, as is currently done. 


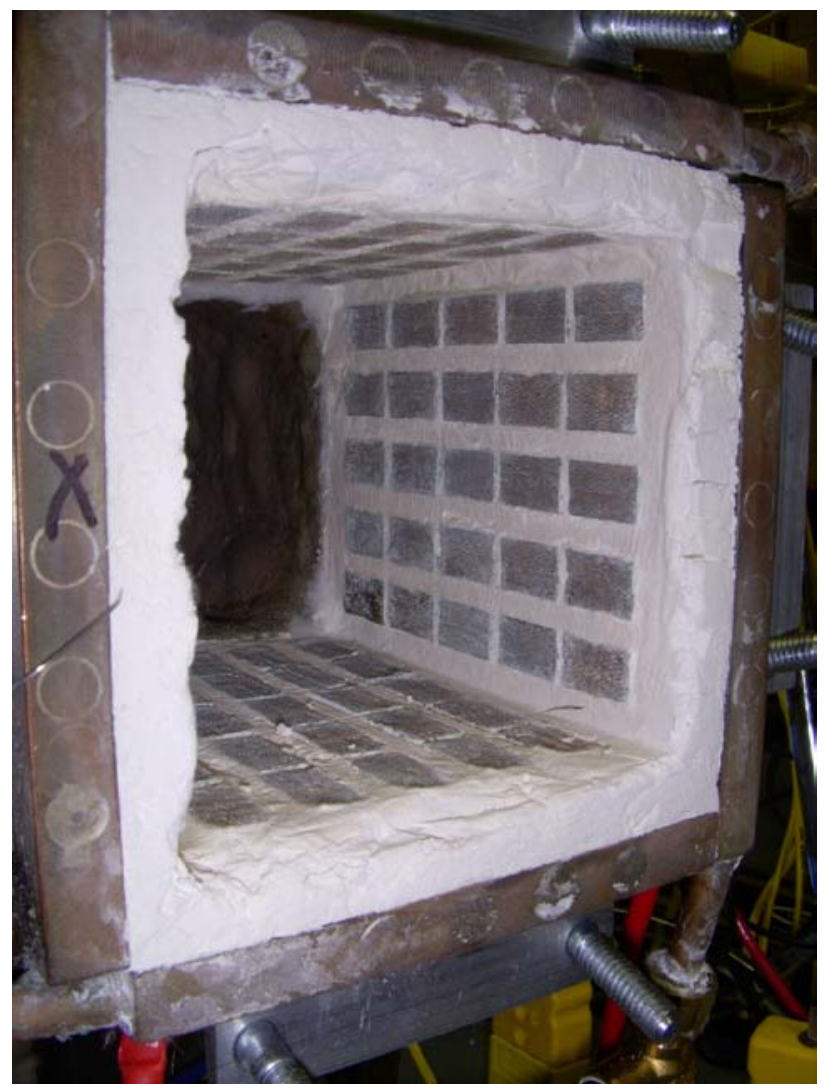

Figure 5.1. First generation TEG insulated with aerogel.

\subsection{Aerogel Thermal Insulation Efficacy}

The thermal insulation efficacy measured the temperature drop of the hot side gas and heating of the cold-side fluid through the TEG panels (Figure 5.2). The data show the heat flux through the 4 different panels of the TEG. Based on predictions, each couple nominally produced 1 watt. Since there were 25 couples per panel this required approximately $300 \mathrm{~W}$ to produce $25 \mathrm{~W}$ per panel or 8\% conversion efficiency at the device level. The panel consisting of TE modules and insulated with commercial wool (Panel 1) had the highest heat flux that was likely attributed to the relatively poor thermal insulation efficacy. Approximately 800 watts passed through Panel 1 , thus if 300 watts passed through the thermoelectric modules, 500 watts were lost as parasitic heat loss. Thermal insulation efficacy is defined as the percentage of heat flowing from the heat source through the thermoelectric modules. For Panel 1, the thermal insulation efficacy was 37.5\%. The panel insulated with aerogel (Panel 2) demonstrated a thermal insulation efficiency of $50 \%$ or is $30 \%$ more efficient than the commercial wool. It is important to note that this was the first generation of aerogel encapsulated TEG modules at this scale. Our process for insulating individual subassemblies and installing them in panels can still be improved. Eliminating the possibility of gaps between insulated subassemblies and the integrating of mica sheets to block gas convection will likely result in significant improvements in thermal insulation efficacy. The goal is to achieve $>90 \%$ thermal insulation efficacy, which is what was achieved for RTG technology operating in space vacuum. 


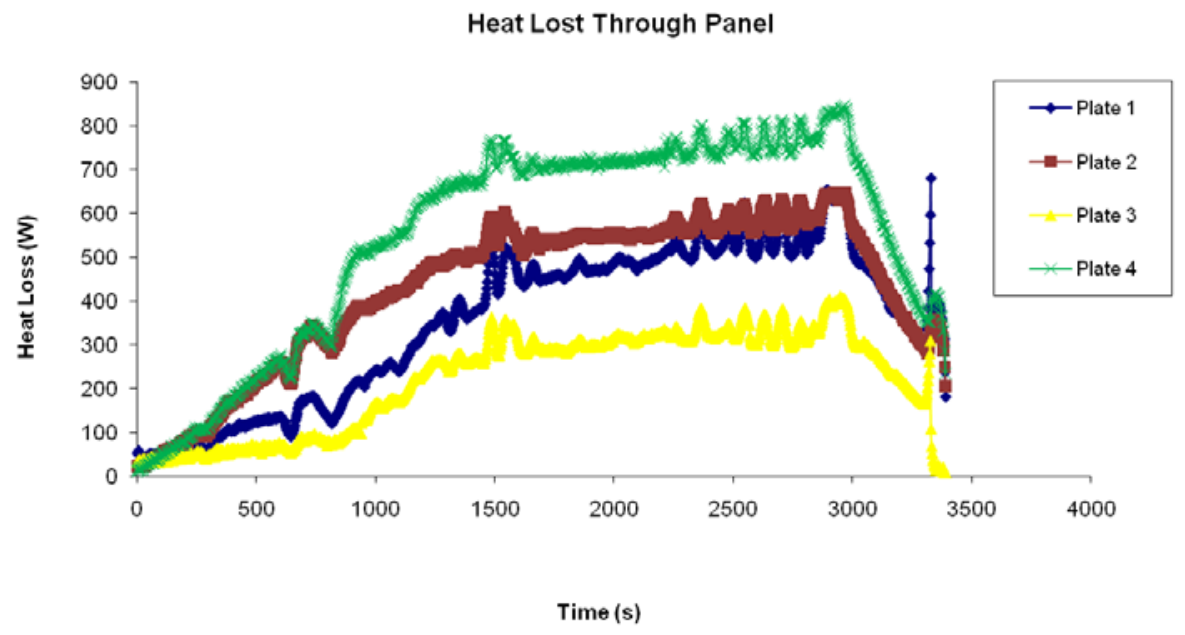

Figure 5.2. Thermal insulation efficacy measurements on a 4-panel TEG (example image). Plate 4-panel with TE modules insulated by commercial wool, Plate 2-panel with TE modules insulated with aerogel, Plates 3 , insulated with commercial wool and mica sheet.

\subsection{Developing the Next Generation Aerogel Insulation}

Several different chemistries have been explored to improve the mechanical properties and castability of the aerogel insulation. The surface of silica aerogels was modified by reaction with chlorotrimethylsilane to make it hydrophobic. Hydrophobic gels were also synthesized using an alternative precursor (methyltrimethoxysilane).

Both attempts were successful in making hydrophobic aerogel (Figure 5.3) By making the insulation hydrophobic it will be more stable in ambient air, thus prevent cracking. The likelihood of it shrinking or pulling away from the TE elements will also be lower. Additionally, making the aerogel hydrophobic may enable the drying of aerogel under ambient conditions, i.e. without a high pressure vessel that is currently used. If successful this process could make aerogel for TEG considerably more cost-effective. It may enable the cost-effective synthesis of aerogel insulation for other bulk-scale thermal insulation applications, such as solid oxide fuel cells and high temperature industrial processes. It is also interesting to note that the gel modified with chlorotrimethylsilane was significantly stronger than all silica aerogels made in the past. Efforts to characterize the compressive modulus are under way. 


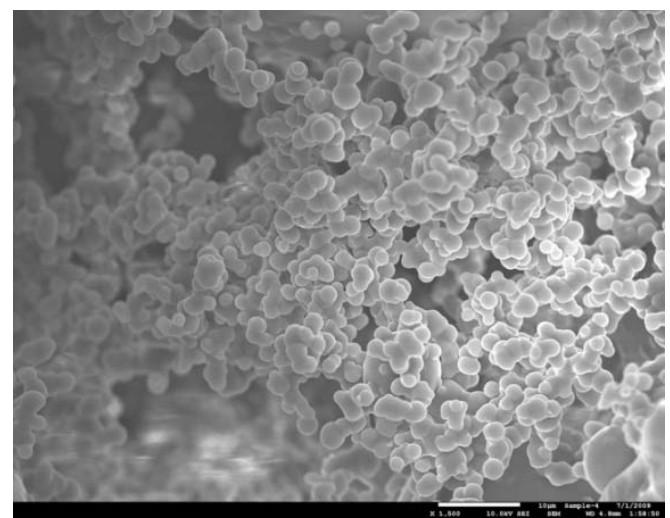

(a)

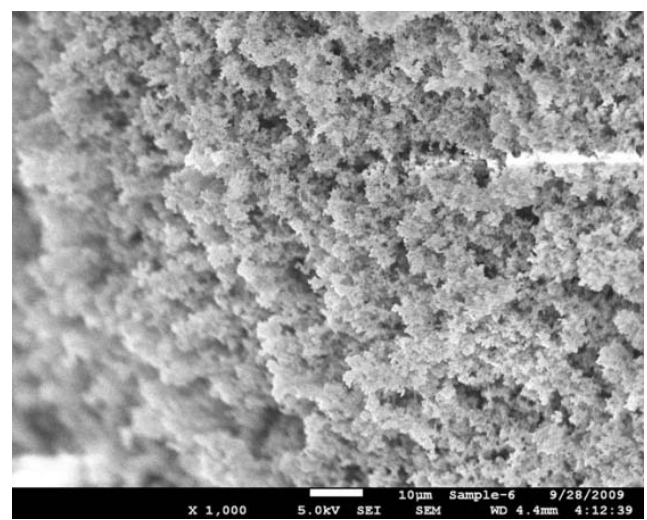

(b)

Figure 5.3. SEM images of chlorotrimethylsilane modified gel (a) and gel made using methyltrimethoxisilane (b).

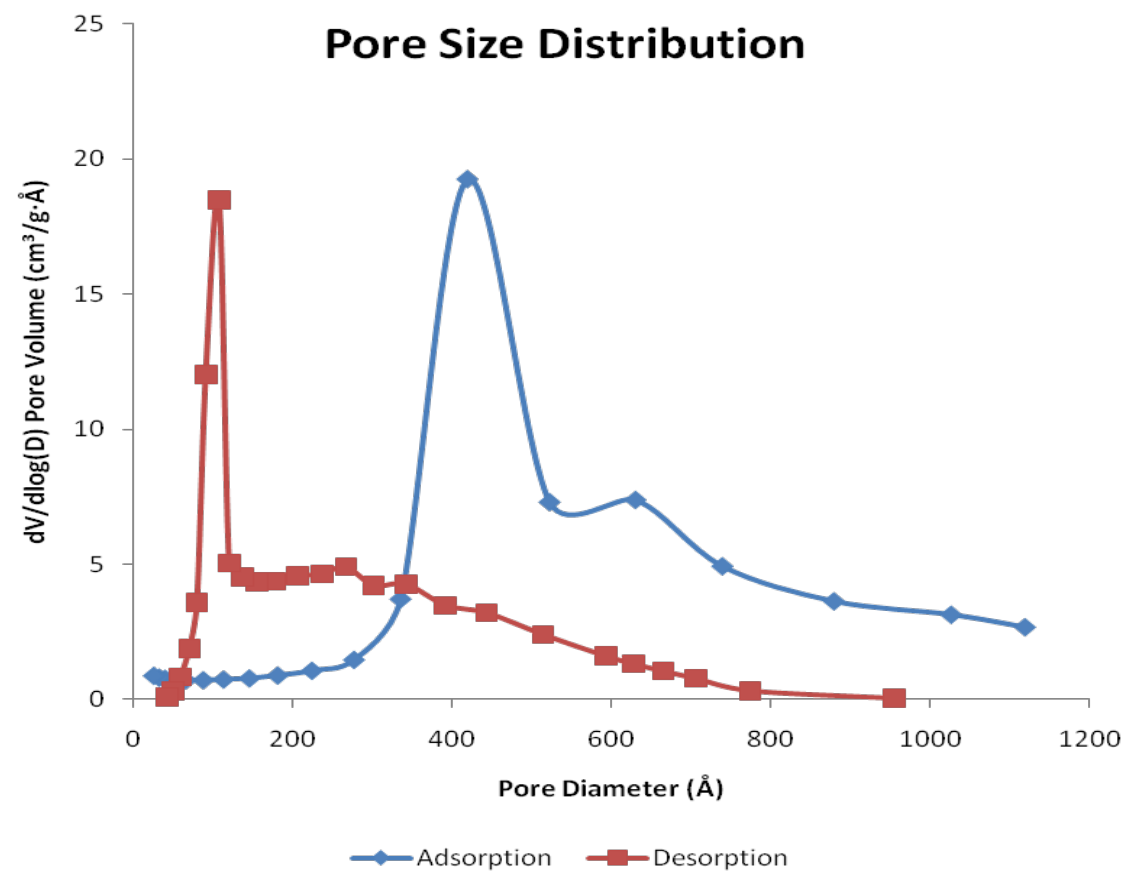

Figure 5.4. Nitrogen absorption characterization of $80 \mathrm{mg} / \mathrm{cc}$ silica. The red data (desorption) is used to quantify the pore volume as a function of pore diameter.

Efforts were also made to make the pores within the aerogel smaller to further reduce the gas convective component of thermal conduction (Figure 5.4). $80 \mathrm{mg} / \mathrm{cc}$ silica aerogel was successfully synthesized. Although the mean diameter is $20 \mathrm{~nm}$, there are a significant number of pores that are approximately $10 \mathrm{~nm}$ in diameter. By decreasing the porosity to below $10 \mathrm{~nm}$ it is believed that a significant reduction in gas convection will result as the flow transitions from Knudsen to activated flow. 


\subsection{Future Work}

Future work will entail efforts to improve thermal insulation efficacy. We will also look at the characterization of the stability of skutterudite at high temperatures in air using aerogel as a protective coating and will also pursue the ability to ambiently dry aerogel. Thermal insulation efficacy will be conducted using pseudo-calorimetric techniques as well as infrared imaging analysis.

\section{TEG Power Electronics Group}

One of the major activities related to the development of the TEG is the power electronics (PE) for the thermoelectric generation. The following accomplishments in PE have been completed:

1. A $1 \mathrm{~kW} \mathrm{12x}$ zero-current-switching (ZCS) multi-level modular switched-capacitor dc-dc converter (MMSCC) prototype has been built and tested.

2. A four-phase interleaved ZCS-MMSCC topology with minimized input current ripple has been proposed and investigated for the $5 \mathrm{~kW}$ voltage boost dc-dc converter prototype.

In this report we will provide the proposed $1 \mathrm{~kW}$ dc-dc converter configuration and the test experimental results, as well as the proposed $5 \mathrm{~kW}$ dc-dc converter topology and simulation results.

\section{1 $1 \mathrm{Kw}$ 12x TEG Voltage Boost Circuit Prototype}

Figure 6.1 shows the $1 \mathrm{~kW} 12 \mathrm{x} 5 \mathrm{~V}$ to $60 \mathrm{~V}$ TEG voltage boost main circuit configuration. $\mathrm{Sr}$ and $\mathrm{Sb}$ are the same switching device with complementary control signal in 50\% duty cycle. A new prototype based on this configuration has been built and tested. Figure 6.2 and Figure 6.3 shows the experiment result.

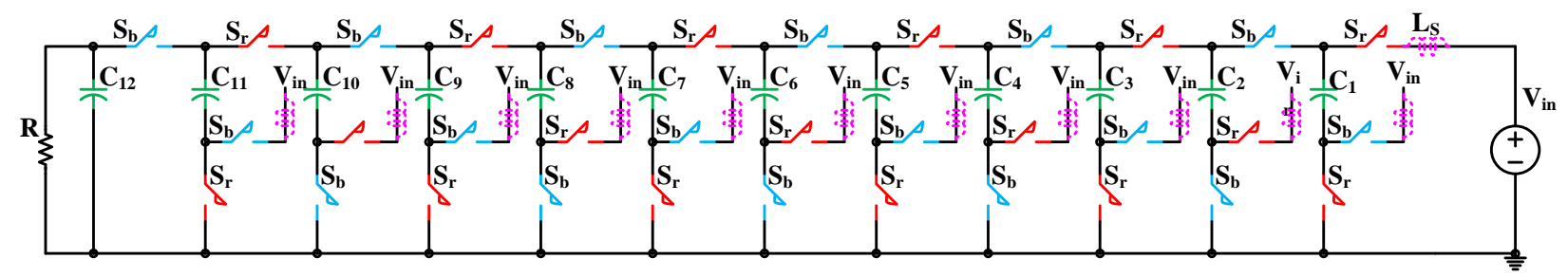

Figure 6.1. $1 \mathrm{~kW} 12 \mathrm{x} 5 \mathrm{~V}$ to $60 \mathrm{~V}$ TEG voltage boost circuit configuration.

\subsection{Four-Phase Interleaved ZCS-MMSCC for 5 kW Prototype}

Figure 6.2 shows the proposed four-phase interleaved ZCS-MMSCC with continuous input current. Because of the interleaved design, the input current ripple was reduced by $80 \%$ than the traditional ZCS-MMSCC. So, the converter efficiency is increased and power rating could be increased to $5 \mathrm{~kW}$. 


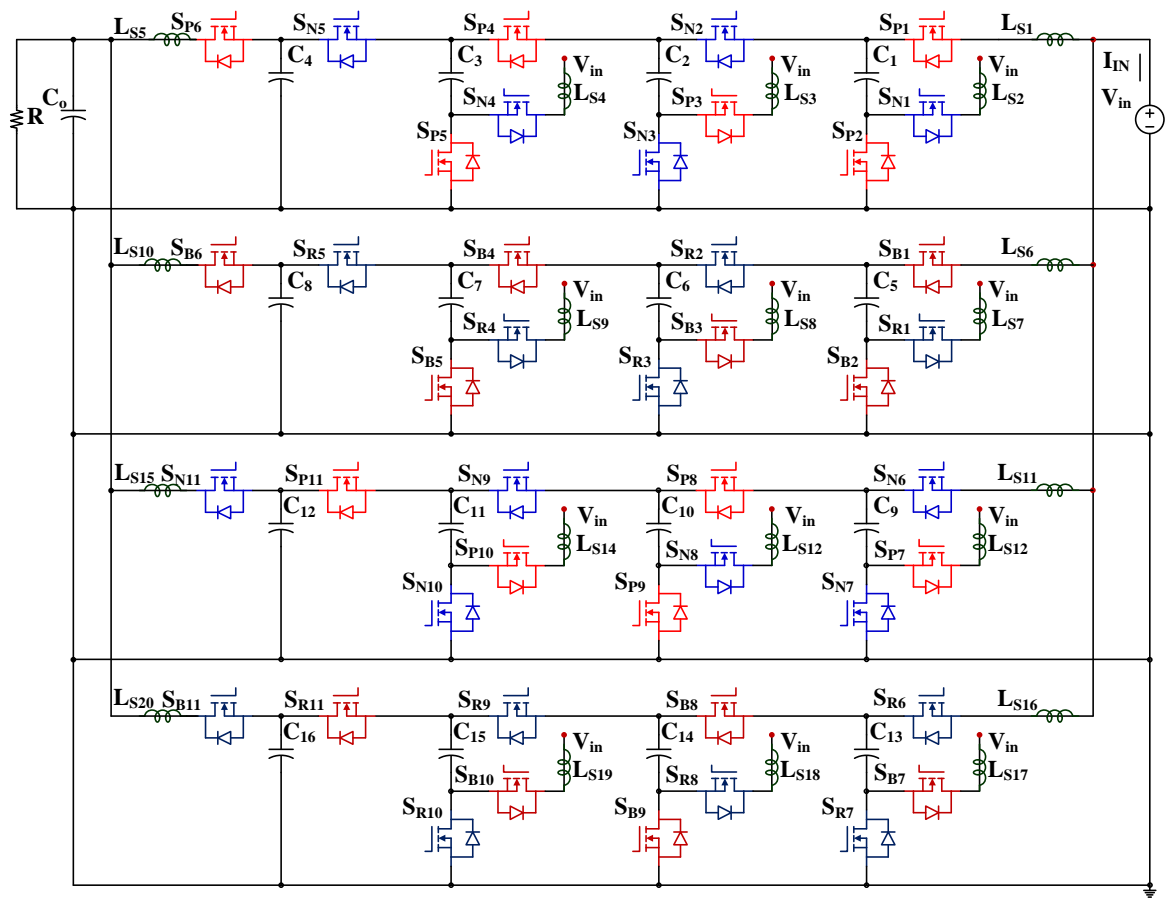

Figure 6.2. Four-phase interleaved ZCS-MMSCC circuit configuration.

Figure 6.3 shows the voltage and current waveforms of switch Sb. The zero current switching has been achieved. Figure 6.4 shows the gate drive voltage and current waveforms of Sb and Sr. It also shows that the two switches are controlled complementarily in a $50 \%$ duty cycle and both switches achieve zero current switching. Figure 6.5 shows the simulation waveforms of the proposed four-phase interleaved ZCS-MMSCC. The input current $\mathrm{I}_{\text {in }}$ becomes continuous and the input current ripple is reduced significantly.

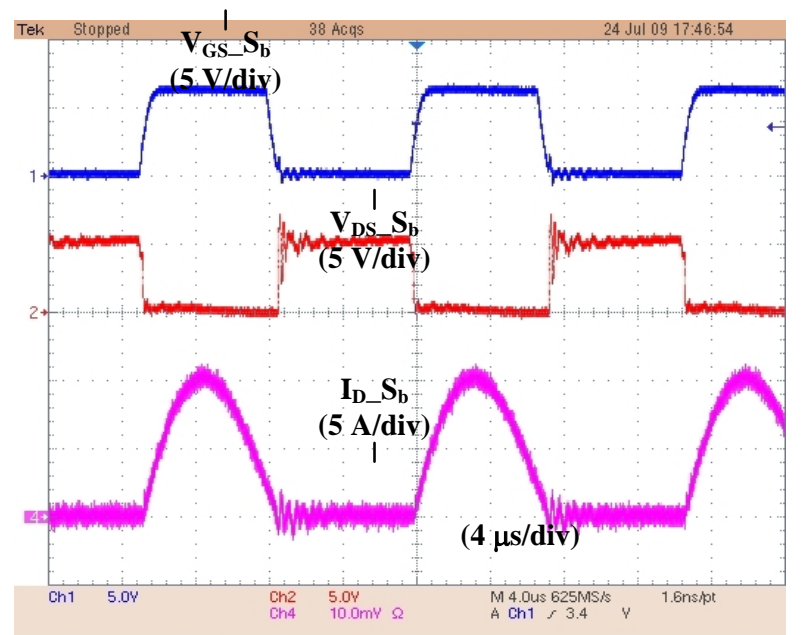

Figure 6.3. Voltage - current waveform of Sb. 


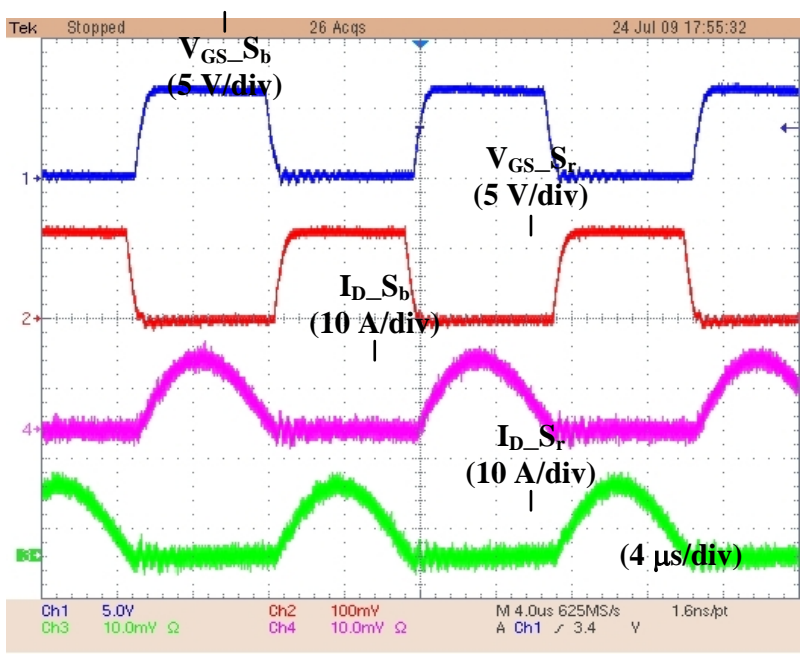

Figure 6.4. Gate drive and current waveforms of Sb and Sr. 


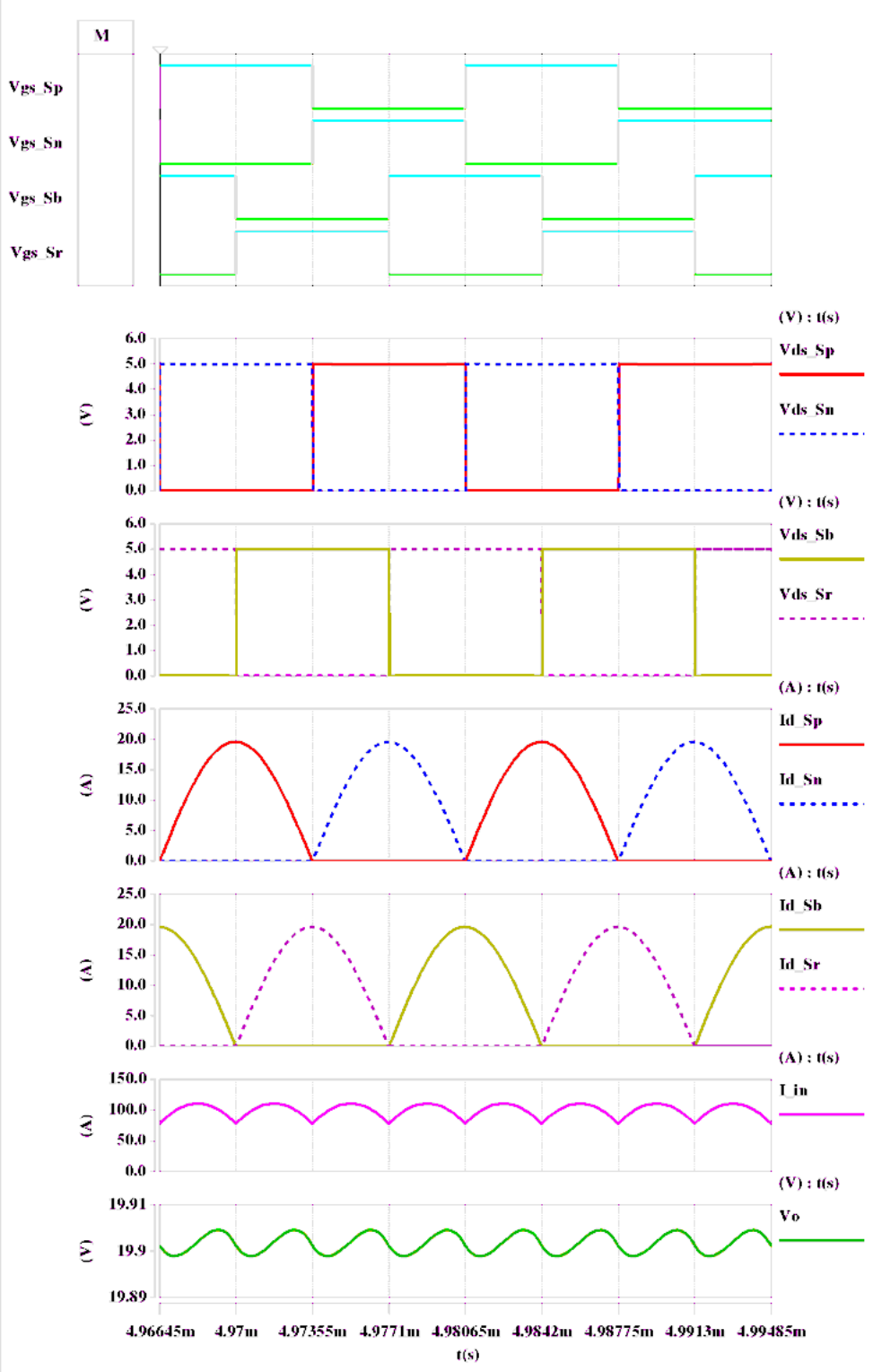

Figure 6.5. Simulation results of four-phase interleaved ZCS-MMSCC.

\subsection{Conclusion and Plans for the Future}

A new $1 \mathrm{~kW} 5 \mathrm{~V}$ input $60 \mathrm{~V}$ output high efficiency high conversion ratio dc-dc converter prototype has been built and tested. A novel $5 \mathrm{~kW}$ high efficiency high gain dc-dc converter topology with continuous input current has been proposed and investigated. A $1 \mathrm{~kW}$ TEG demo system with voltage boost and faulty module bypass function will be built and tested using the new $1 \mathrm{~kW}$ prototype. 


\section{Cost and Price Model of $1 \mathrm{~kW}$ and 5kW Thermoelectric Based Auxiliary Power Units}

\section{- Class 8 Trucks}

\subsection{Introduction}

This study is intended to serve as an initial look at the economics associated with the use of a waste heat energy recovery system that also provides an auxiliary power unit to the vehicle utilizing solid state thermoelectric generators. To facilitate the cost and pricing study the auxiliary power unit (APU) under study may be divided up into four major subsystems:

- Electrical/Electronics

- Thermoelectric Generator

- Burner

- Cooling Subsystem.

In developing a "retail” price for the whole system a process of establishing a price for each of the subsystems and adding the parts up to establish the total price was used. The price number reflects an attempt to include such things as fully accounted for cost of labor, overhead (building cost, cost of capital, etc.), general and administrative cost. Generally, the material costs make up only a certain percentage of the price and the specific percentage varies by subsystem. Thus by having the material cost and the percent of the price we can calculate the price of the part.

The early estimate for the price a manufacturer would have to charge for a $1 \mathrm{~kW}$ system to cover his cost and provide a reasonable return is $\$ 4,773.77$. The estimate for the price of a $5 \mathrm{~kW}$ system using the same procedure as for the $1 \mathrm{~kW}$ system is $\$ 19,276.13$. The details for developing these price estimates follow.

\subsection{Electrical/Electronics}

The electronic subsystem is the most mature of the design efforts and allowed for the most straightforward costing study. Bill of Materials (BOM) associated with a $1 \mathrm{~kW}$ and a $5 \mathrm{~kW}$ designs were created by the electronics group. Table 7.1 contains the BOM for the $1 \mathrm{~kW}$ unit and Table 7.2 contains the BOM for the $5 \mathrm{~kW}$ unit. All control features associated with the system are assumed to be contained in the single electronics module. 
Table 7.1. Bill of material for $1 \mathrm{~kW}$ unit.

\begin{tabular}{|c|c|c|}
\hline Part Type & Manuf. Part No. & Manufacturer \\
\hline $\begin{array}{c}\text { Electrically Activated } \\
\text { Switch }\end{array}$ & - & - \\
\hline Capacitor & C5750X7R2A475M & TDK \\
\hline Capacitor & PLG1C471MDO1 & Nichicon \\
\hline PCB board & PCB(56 in^2 each) & Pcbfabexpress \\
\hline Resistor & CFR-50JB-2R4 & Yageo \\
\hline Capacitor & PLF1D151MDO2 & Nichicon \\
\hline Diode & 1N5819 & Vishay IR \\
\hline Power supply & PT5041N & TI \\
\hline Voltage regulator & L7805CV & STMicroelectronics \\
\hline Capacitor & PLE0J471MDO1 & Nichicon \\
\hline $\begin{array}{c}\text { Electrically Activated } \\
\text { Switch }\end{array}$ & - & - \\
\hline Core & T90-70 & Micrometals \\
\hline Wire & 7304K153 & McMaster \\
\hline Ring Terminal & 7113K18 & McMaster \\
\hline PCB board & PCB & Pcbfabexpress \\
\hline Microcontroller & DSPIC33FJ16GS502-E/SO & Microchip \\
\hline
\end{tabular}

Table 7.2. Bill of material for $5 \mathrm{~kW}$ unit.

\begin{tabular}{|c|c|c|}
\hline Part Type & Manuf. Part No. & Manufacturer \\
\hline $\begin{array}{c}\text { Electrically Activated } \\
\text { Switch }\end{array}$ & - & - \\
\hline Capacitor & C5750X7R2A475M & TDK \\
\hline Capacitor & PLG1C471MDO1 & Nichicon \\
\hline PCB board & $\begin{array}{c}\text { 32cm*60cm two } \\
\text { board,double layer }\end{array}$ & Pcbfabexpress \\
\hline Resistor & CFR-50JB-2R4 & Yageo \\
\hline Capacitor & PLF1D151MDO2 & Nichicon \\
\hline Diode & 1N5819 & Vishay IR \\
\hline Power supply & PT5044N & TI \\
\hline Voltage regulator & L7805CV & STMicroelectronics \\
\hline Capacitor & PLE0J471MDO1 & Nichicon \\
\hline Core & T90-70 & Micrometals \\
\hline Wire & 7304K153 & McMaster \\
\hline Ring Terminal & 7113K18 & McMaster \\
\hline PCB board & $\begin{array}{c}\text { 6in*3in one board } \\
\text { double layer }\end{array}$ & Pcbfabexpress \\
\hline Microcontroller & $\begin{array}{c}\text { DSPIC33FJ16GS502- } \\
\text { E/SO }\end{array}$ & Microchip \\
\hline
\end{tabular}

Three different volume scenarios $(10,000,50,000$ and 250,000) were studied to identify cost sensitivity to volume. For the $1 \mathrm{~kW}$ unit, the low volume prototype costs are each $\$ 1571.45$. The 10,000 unit cost is $\$ 471.64$ for each. The 50,000 unit cost is $\$ 448.23$ for each unit and the 250,000 unit cost is $\$ 444.03$. Clearly, there is little cost reduction with volumes higher than 
10,000 units. It is a similar story for the $5 \mathrm{~kW}$ unit. The low volume prototype cost for the $5 \mathrm{~kW}$ unit is $\$ 5662.49$. The 10,000 unit cost is $\$ 2335.84$ for each unit. The 50,000 unit cost is $\$ 2245.79$ for each one and the 250,000 unit cost is $\$ 2229.75$ for each one.

If we assume a 10,000 annual volume, this is a relatively low manufacturing volume for electronics and as such it is likely that the price of the BOM is $50 \%$ of the selling price. That is, the price for the $1 \mathrm{~kW}$ unit would be $\$ 943.28$ and the price for the $5 \mathrm{~kW}$ unit would be $\$ 4671.68$.

\subsection{Thermoelectric Generator}

The thermoelectric generator (TEG) subsystem is the heart of the whole system and is the subject of the major research on this project. The TEG design maturity is the least developed and therefore is the biggest challenge from a cost study point of view. The TEG subsystem may be further subdivided into three parts: the thermoelectric junctions, the 1000 Watt TEG module assemblies; and the housing for packaging of the assemblies.

\subsection{Thermoelectric Junctions}

JPL has performed a basic material cost study looking at world markets for the materials associated with the different possible junction chemistries. Table $\mathbf{7 . 3}$ is a summary of their findings.

Table 7.3. JPL cost estimates

\begin{tabular}{|c|c|c|c|c|c|}
\hline Material & \$ per kg & Max Thot (K) & $\mathbf{Z T}_{\text {ave }}$ & $\mathbf{W} / \mathbf{k g}$ & $\mathbf{\$} / \mathbf{W}$ \\
\hline $\mathrm{Si}_{80} \mathrm{Ge}_{20}$ & 630 & 1275 & 0.49 & 63 & 9.9 \\
\hline $\mathrm{Si}_{98} \mathrm{Ge}_{2}$ & 83 & 1275 & 0.32 & 49 & 1.7 \\
\hline $\mathrm{Mg}_{2} \mathrm{Si}_{0.6} \mathrm{Sn}_{0.4}$ & 11 & 750 & 0.45 & 58 & 0.2 \\
\hline $\mathrm{CoSb}_{3}$ & 18 & 873 & 0.82 & 73 & 0.2 \\
\hline $\mathrm{TAGS}$ & 631 & 673 & 0.77 & 172 & 3.7 \\
\hline $\mathrm{PbTe}$ & 83 & 800 & 0.63 & 86 & 1.0 \\
\hline $\mathrm{Bi}_{2} \mathrm{Te}_{3}$ & 170 & 525 & 0.79 & 232 & 0.7 \\
\hline
\end{tabular}

From this study, we see a wide range of the potential cost per Watt. These range from a high of $\$ 9.90$ per Watt to a low of $\$ .20$ per Watt. For the purposes of our cost study, we will use the lowest cost per Watt number because if the economics do not work out with these optimistic numbers they will not work out with the higher numbers.

With the cost of $\$ .20$ per Watt, we need both P-type as well as N-type junctions. This means that the cost for a $1 \mathrm{~kW}$ unit is $\$ 400.00$. The cost for the $5 \mathrm{~kW}$ unit is 5 times the cost of the $1 \mathrm{~kW}$ unit $(\$ 2,000)$.

The manufacturing process for the junctions is relatively labor intensive and utilizes some nonstandard manufacturing equipment. Henceforth, the cost of the material makes up one-third of the price of the system. Thus, the price of the junctions associated with a $1 \mathrm{~kW}$ unit would be $\$ 1200$ and the price of the junctions associated with a $5 \mathrm{~kW}$ unit would be $\$ 6,000$. As the manufacturing process and chemistries become better defined more precise pricing estimates will be generated. 


\subsection{TEG Module Assemblies}

There are approximately eight major parts that make up the module assembly. The MSU team assembled the current costs associated with each of the parts and this served as the starting point to develop the production cost estimates. For example, the copper hot side heat coupler is currently a machined copper part. However, in production this part could be an extrusion or a medal injected molded part. The MSU cost for this part is $\$ .17742$ per piece or $\$ 177.42$ per 1 $\mathrm{kW}$ assembly. If we assume that a high volume manufacturing process is used then a cost reduction of up to one-third of the low volume cost may be possible ( $\$ 60.00$ per $1 \mathrm{~kW}$ unit). The cost of the $5 \mathrm{~kW}$ unit is just 5 times the cost of the $1 \mathrm{~kW}$ unit $(\$ 300.00)$.

Because of the high operating temperatures involved the unit uses silver solder as a means of attachment on the hot side parts. As the cost of silver solder is largely set by the world market price of silver, the MSU volume cost reduction of $25 \%$ is possible. Therefore, the cost $(.75$ * $\$ 97.61)$ is $\$ 73.21$ for the $1 \mathrm{~kW}$ unit and the $5 \mathrm{~kW}$ cost is 5 times the $1 \mathrm{~kW}$ cost (\$366.05).

Regular solder is used for attaching low temperature items in the assembly. Again, MSU volume cost reduction of $25 \%$ is possible. The $1 \mathrm{~kW}$ unit the cost $(.75 * \$ 12.43)$ is $\$ 9.32$. The cost for the $5 \mathrm{~kW}$ unit is 5 times the cost for the $1 \mathrm{~kW}$ unit $(\$ 46.60)$.

The copper cold side coupler (supplied by MSU) is essentially a flat copper sheet stock. The cost for the $1 \mathrm{~kW}$ unit is $\$ 26.14$ and the cost for the $5 \mathrm{~kW}$ unit is $\$ 130.70$.

Epoxy is used as a mechanical fastener within the assembly. We can assume that we may obtain a reasonable MSU volume discount of 50\%. The epoxy cost for the $1 \mathrm{~kW}$ unit $(.5 * \$ 279.60)$ is $\$ 139.75$. The epoxy cost for the $5 \mathrm{~kW}$ unit is $\$ 698.75$. We might consider a lower grade epoxy or use less of the epoxy as a possible cost reduction.

The assembly uses an aluminum base plate. Again there is a potential volume discount of $50 \%$ from the cost used in the low volume prototypes assemblies. Therefore, the cost of the aluminum base plate for the $1 \mathrm{~kW}$ unit $(.5 * \$ 14.89)$ is $\$ 7.45$. The cost of the aluminum base plate for the $5 \mathrm{~kW}$ unit is $\$ 37.25$.

Brass bolts are utilized as mechanical connectors in the current design. We can assume a $50 \%$ reduction in the MSU cost for the brass bolts. The cost for the brass bolts in the $1 \mathrm{~kW}$ unit $(.5 *$ $\$ 31.24)$ is $\$ 15.62$. The cost of the brass bolts for the $5 \mathrm{~kW}$ unit is $\$ 78.10$.

The last major part of the TEG module assembly is the aerogel insulation. The MSU low volume cost for the prototypes is $\$ 108.66$ for the $1 \mathrm{~kW}$ unit. This is a major candidate for a $60 \%$ reduction. Therefore, the cost of the aerogel insulation for the $1 \mathrm{~kW}$ unit $(.4 * \$ 108.66)$ is $\$ 43.46$. The cost associated with the aerogel insulation in the $5 \mathrm{~kW}$ unit is $\$ 217.30$.

In summary, the costs associated with the eight major parts of the $1 \mathrm{~kW}$ module assembly (not including the cost of the junctions), includes the following: 


\begin{tabular}{|c|c|}
\hline Copper hot side heat coupler & $\$ 60.00$ \\
\hline Silver solder & $\$ 73.21$ \\
\hline Solder & $\$ 9.3$ \\
\hline Copper cold side coupler & $\$ 26.1$ \\
\hline Epoxy & $\$ 139.7$ \\
\hline Aluminum base plate & $\$ 7.4$ \\
\hline Brass bolts & $\$ 15.6$ \\
\hline Aerogel & $\$ 43$. \\
\hline
\end{tabular}

This does not include the manufacturing costs. Therefore, it is reasonable to use a pricing model where the cost is only one-third of the final price. The price associated with the $1 \mathrm{~kW}$ TEG module assemble (not including the junction) is $\$ 1124.85$. The price associated with the $5 \mathrm{~kW}$ TEG module assembly (not including the junction) is $\$ 5624.25$. As the manufacturing process becomes better defined we will be able to provide more precise pricing estimates.

\subsection{Housing}

Finally, the third element which makes up the TEG subsystem is the housing for packaging of everything. A search for a stainless steel Class 8 truck muffler on the internet identified several units within the retail price range of approximately $\$ 400$. This will be used for the price build up of the $1 \mathrm{~kW}$ unit. The $5 \mathrm{~kW}$ unit is considerably larger, therefore we will use a multiplier of 1.5 in setting the pricing model for the $5 \mathrm{~kW}$ unit $(\$ 600)$.

\subsection{Burner}

The engine will be stopped when the vehicle is parked for extended periods of time. To provide heat energy to the TEG system to generate electricity a Diesel fuel burner is utilized as part of the overall system. The unit chosen is manufactured by Heatwise Inc. The Pioneer $1 \mathrm{k}$ has a retail price of $\$ 507$.

A valve is needed to control the flow of exhaust heat in the hot exhaust stream. As a surrogate for this valve design, an automotive grade Diesel exhaust gas recirculation valve was used. An internet search revealed a typical retail price of approximately $\$ 190$ for this valve.

Finally, a section of stainless steel exhaust tubing is needed to interconnect the heater into the engine exhaust TEG system. A price of \$20 is assumed for this tubing. The total price for both the $1 \mathrm{~kW}$ and $5 \mathrm{~kW}$ burner subsystems is $\$ 717$.

\subsection{Cooling Subsystem}

There are 4 major elements which go into the makeup of the cooling subsystem. Cooling plates couple the coolant to the cold side of the TEG module assembly and provide a mechanism to maintain the maximum delta temperature across the thermoelectric junction. Cooling lines couple the cooling plates to the external heat exchanger and a pump to move the coolant.

The MSU cost associated with the cooling plates for the $1 \mathrm{~kW}$ unit is $\$ 288.63$. If we assume that a design consistent with high volume manufacturing is developed we could expect a $50 \%$ 
reduction in this cost. Therefore, the cost associated with the cooling plates for the $1 \mathrm{~kW}$ unit $(.5$ * 288.63) is $\$ 144.32$. The cost of the cooling plates for the $5 \mathrm{~kW}$ unit scales linearly with power. Therefore the cost of the cooling plates for the $5 \mathrm{~kW}$ unit is $\$ 721.60$.

We assume that automotive grade heater cooling hose is acceptable and the cost for the hose and associated clamps is $\$ 10.00$ for the $1 \mathrm{~kW}$ unit. As the number of hoses is proportional to the power, the cost for the hoses and clamps for the $5 \mathrm{~kW}$ unit is $\$ 50.00$.

For a heat exchanger, an automotive grade heater core is used as a surrogate. The cost of a typical heater core is approximately $\$ 15.00$. To scale up the cost for the $5 \mathrm{~kW}$ system, a heater core cost of $\$ 22.50$ is used.

Finally, it is assumed that an automotive grade coolant pump will be used. A cost of a $1 \mathrm{~kW}$ unit is $\$ 25.00$ and $\$ 37.50$ for the $5 \mathrm{~kW}$ unit.

Total cost for the cooling subsystem associated with the $1 \mathrm{~kW}$ unit is $\$ 194.32$. We assume that the cost makes up 50\% of the subsystem price and equals $\$ 388.64$.

Total cost for the cooling subsystem associated with the $5 \mathrm{~kW}$ unit is $\$ 831.60$. We assume that the cost makes up 50\% of the subsystem price and equals \$1663.20.

\subsection{Total $1 \mathrm{~kW}$ System Price}

To develop the price for the $1 \mathrm{~kW}$ system we totaled the prices for the following 4 major subsystems:

\begin{tabular}{|c|c|}
\hline \\
\hline & $\begin{array}{l}\text { Electrical/Electron } \\
\text { TEG Subsystem: }\end{array}$ \\
\hline \multicolumn{2}{|l|}{ TEG devices } \\
\hline \multicolumn{2}{|l|}{ Module Assembly } \\
\hline $\begin{array}{l}\text { Module Assembly } \\
\text { Housing }\end{array}$ & $\$ 400.00$ \\
\hline \multicolumn{2}{|l|}{ Burner } \\
\hline \multirow{2}{*}{\multicolumn{2}{|c|}{ Cooling Subsystem }} \\
\hline & $\$ 4,773.77$ \\
\hline
\end{tabular}

\subsection{Total 5 kW System Price}

To develop the price for the $5 \mathrm{~kW}$ system, we total the prices for the following 4 major subsystems:

\begin{tabular}{|c|c|}
\hline \multirow{2}{*}{\multicolumn{2}{|c|}{$\begin{array}{l}\text { Electrical/Electronics } \\
\text { TEG Subsystem: }\end{array}$}} \\
\hline & \\
\hline TEG devices & $\$ 6000.00$ \\
\hline Module Assembly & $\$ 5624.25$ \\
\hline Housing & $\$ 600.00$ \\
\hline Burner & $\$ 717.00$ \\
\hline Cooling Subsystem & $\$ 1663.20$ \\
\hline Total & $\$ 19,276.13$ \\
\hline
\end{tabular}


These prices are clearly estimates. As the design and process steps become better defined the cost and prices can be reduced.

\section{Material Research, Mechanical Properties and Thermoelectric Property Measurements}

In addition to the development and fabrication of the TEG, our research also focused on parallel studies that could be useful in providing materials that may be helpful in increasing the efficiency of the device. Detailed studies of the mechanical properties of the thermoelectric material are important in the design of the TEG. Methods to reduce grain size in hot pressed material are significant to increasing the strength of the thermoelectric material. Significant research on the reduction of particle size in the thermoelectric powder is presented.

\subsection{Material Research - LAST and LASTT Cast Materials}

Simulation of the output power from the thermoelectric generators are based on the typical material properties of the samples used to fabricate those generators. A compilation of temperature dependent electrical conductivity $(\sigma)$ and thermopower $(S)$, for cast samples of $\mathrm{N}$ type $\mathrm{AgPb}_{m} \mathrm{SbTe}_{2+m}(m=19$, and 22) are shown in Figure 8.1, along with the curve fit to the data (solid black line) used in the modeling software. The measurements and simulations are beneficial to both the DOE and the ONR-MURI programs. However, final device designs and fabrication must be tailored to the specific temperatures and desired heat flows for the system utilizing the thermoelectric generators.

The data shows an electrical conductivity that decreases with increasing temperature. This is indicative of metallic or degenerately doped semiconductor behavior. The data was collected by Bruce Cook at Ames Lab, and Hsin Wang at ORNL and in good agreement with the data collected at Michigan State University. The electrical resistivity, $\rho$, can be fit to a temperature power law as:

$$
\rho=\frac{1}{\sigma}=A \cdot T^{n}
$$

where $A$ is a constant and $n$ was found to range from 1.76 to 2.41 for these samples. The thermopower is relatively linear as a function of temperature, especially for the $m=22$ samples, and have slopes in the range of $0.33\left(\mu \mathrm{V} / \mathrm{K}^{2}\right)$ to $0.40\left(\mu \mathrm{V} / \mathrm{K}^{2}\right)$. The unitless figure of merit, $\mathrm{ZT}$, is

$$
Z T=\frac{S^{2} \sigma}{\kappa} T
$$

where $\kappa$ is the thermal conductivity, and the numerator, $S^{2} \sigma$, is called the power factor. Figure 8.2 and Figure 8.3 show that the power factor begins near $10\left(\mu \mathrm{W} / \mathrm{cm} \cdot \mathrm{K}^{2}\right)$ at room temperature and rises above $15\left(\mu \mathrm{W} / \mathrm{cm} \cdot \mathrm{K}^{2}\right)$ at $700 \mathrm{~K}$ for the $m=22$ samples, while for the $m=19$ samples the power factor begins near $25\left(\mu \mathrm{W} / \mathrm{cm} \cdot \mathrm{K}^{2}\right)$ at room temperature and drops to approximately $15\left(\mu \mathrm{W} / \mathrm{cm} \cdot \mathrm{K}^{2}\right)$ at $700 \mathrm{~K}$.

To examine the influence of the cooling profiles used during fabrication of these materials, data for the two compositions are separately shown in Figure 8.2 and Figure 8.3. They show the data for the $\mathrm{Ag}_{0.43} \mathrm{~Pb}_{18} \mathrm{Sb}_{1.2} \mathrm{Te}_{20}(m=22)$ samples with the data set colors coordinated to the cooling 
profiles used during fabrication. Doping variations between samples would lead to changes in both thermopower and electrical conductivity; the thermopower decreases in magnitude as the electrical conductivity increases. Since the thermopower is an open circuit measurement (zero current flow), microcracking and/or porosity in these brittle samples would cause larger decreases in the electrical conductivity than in the thermopower. This would be consistent with what is shown in Figure 8.2 and in Figure 8.3 for the $\mathrm{Ag}_{0.86} \mathrm{~Pb}_{19} \mathrm{Sb}_{1.0} \mathrm{Te}_{20}(m=19)$ samples. The lower $m$ value materials show larger magnitude thermopower. A general trend of the more rapidly cooled samples showing lower power factors can also be seen. The range of measured power factor values is highlighted with dashed lines.

To determine the figure of merit, ZT, the temperature dependent thermal conductivity was found by first measuring the thermal diffusivity, $D$, and the specific heat capacity, $C_{p}$, and calculating the thermal conductivity, $\kappa$, according to the following equation

$$
\kappa=\rho_{d} D C_{p}
$$

where $\rho_{d}$ is the density of the sample. Thermal diffusivity measurements were taken at ORNL and at Northwestern University. They show good agreement (Figure 8.4). The maximum and minimum trends in the thermal conductivities for this set of samples are also highlighted (dashed green lines in Figure 8.4). 

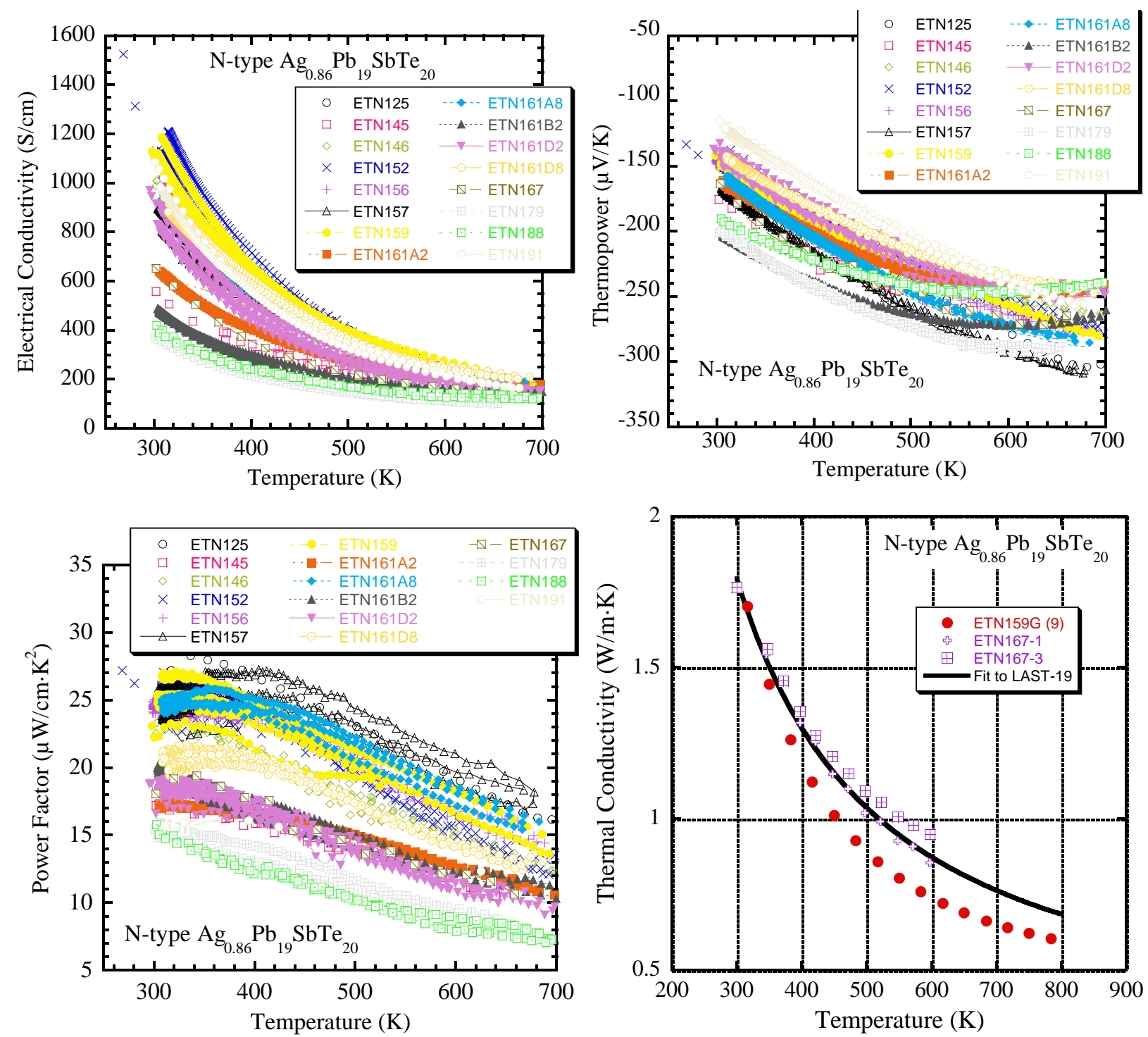

Figure 8.1. Compilation of transport measurements on $\mathrm{N}$-type $\mathrm{Ag}_{0.86} \mathrm{~Pb}_{19} \mathrm{SbTe}_{20}$ samples. 

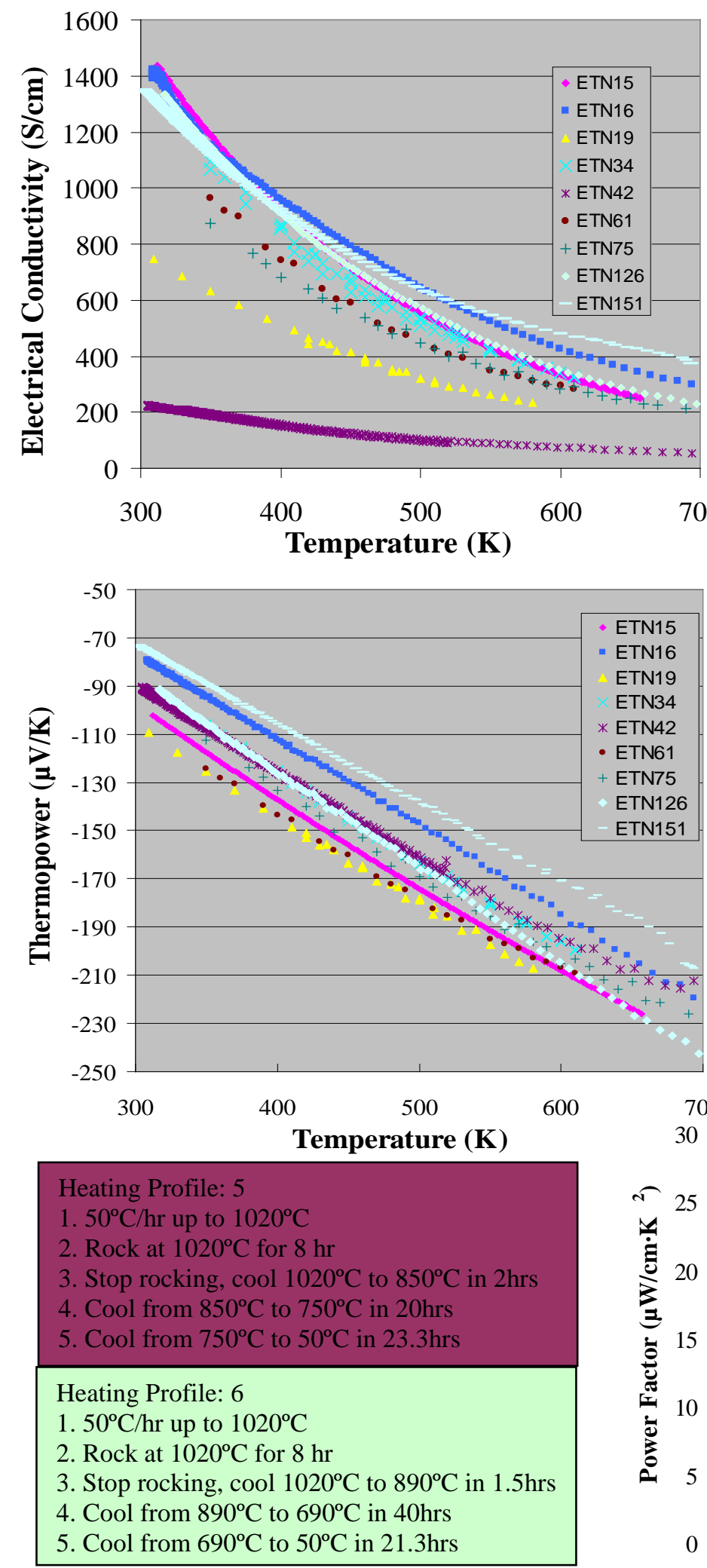

Heating Profile: 1

1. $50^{\circ} \mathrm{C} / \mathrm{hr}$ up to $1020^{\circ} \mathrm{C}$

2. Rock at $1020^{\circ} \mathrm{C}$ for $8 \mathrm{hr}$

3. Stop rocking, cool $1020^{\circ} \mathrm{C}$ to $780^{\circ} \mathrm{C}$ in $24 \mathrm{hrs}$

4. Cool from $780^{\circ} \mathrm{C}$ to $50^{\circ} \mathrm{C}$ in $18.25 \mathrm{hrs}$

Heating Profile: 2

1. $50^{\circ} \mathrm{C} / \mathrm{hr}$ up to $1020^{\circ} \mathrm{C}$

2. Rock at $1020^{\circ} \mathrm{C}$ for $8 \mathrm{hr}$

3. Stop rocking, cool $1020^{\circ} \mathrm{C}$ to $850^{\circ} \mathrm{C}$ in 2 hrs

4. Cool from $850^{\circ} \mathrm{C}$ to $800^{\circ} \mathrm{C}$ in $12 \mathrm{hrs}$

5. Cool from $800^{\circ} \mathrm{C}$ to $400^{\circ} \mathrm{C}$ in 12 hrs

6. Cool from $400^{\circ} \mathrm{C}$ to $50^{\circ} \mathrm{C}$ in 7 hrs

Heating Profile: 3

1. $50^{\circ} \mathrm{C} / \mathrm{hr}$ up to $1020^{\circ} \mathrm{C}$

2. Rock at $1020^{\circ} \mathrm{C}$ for $8 \mathrm{hr}$

3. Stop rocking, cool $1020^{\circ} \mathrm{C}$ to $950^{\circ} \mathrm{C}$ in $1 \mathrm{hr}$

4. Cool from $950^{\circ} \mathrm{C}$ to $850^{\circ} \mathrm{C}$ in 4 hrs

5. Cool from $850^{\circ} \mathrm{C}$ to $780^{\circ} \mathrm{C}$ in $14 \mathrm{hrs}$

6. Cool from $780^{\circ} \mathrm{C}$ to $50^{\circ} \mathrm{C}$ in $24.3 \mathrm{hrs}$

Heating Profile: 4

1. $50^{\circ} \mathrm{C} / \mathrm{hr}$ up to $1020^{\circ} \mathrm{C}$

2. Rock at $1020^{\circ} \mathrm{C}$ for $8 \mathrm{hr}$

3. Stop rocking, cool $1020^{\circ} \mathrm{C}$ to $850^{\circ} \mathrm{C}$ in $2 \mathrm{hrs}$

4. Cool from $850^{\circ} \mathrm{C}$ to $780^{\circ} \mathrm{C}$ in $14 \mathrm{hrs}$

5. Cool from $780^{\circ} \mathrm{C}$ to $400^{\circ} \mathrm{C}$ in 10.9 hrs

6. Cool from $400^{\circ} \mathrm{C}$ to $50^{\circ} \mathrm{C}$ in $7 \mathrm{hrs}$ 

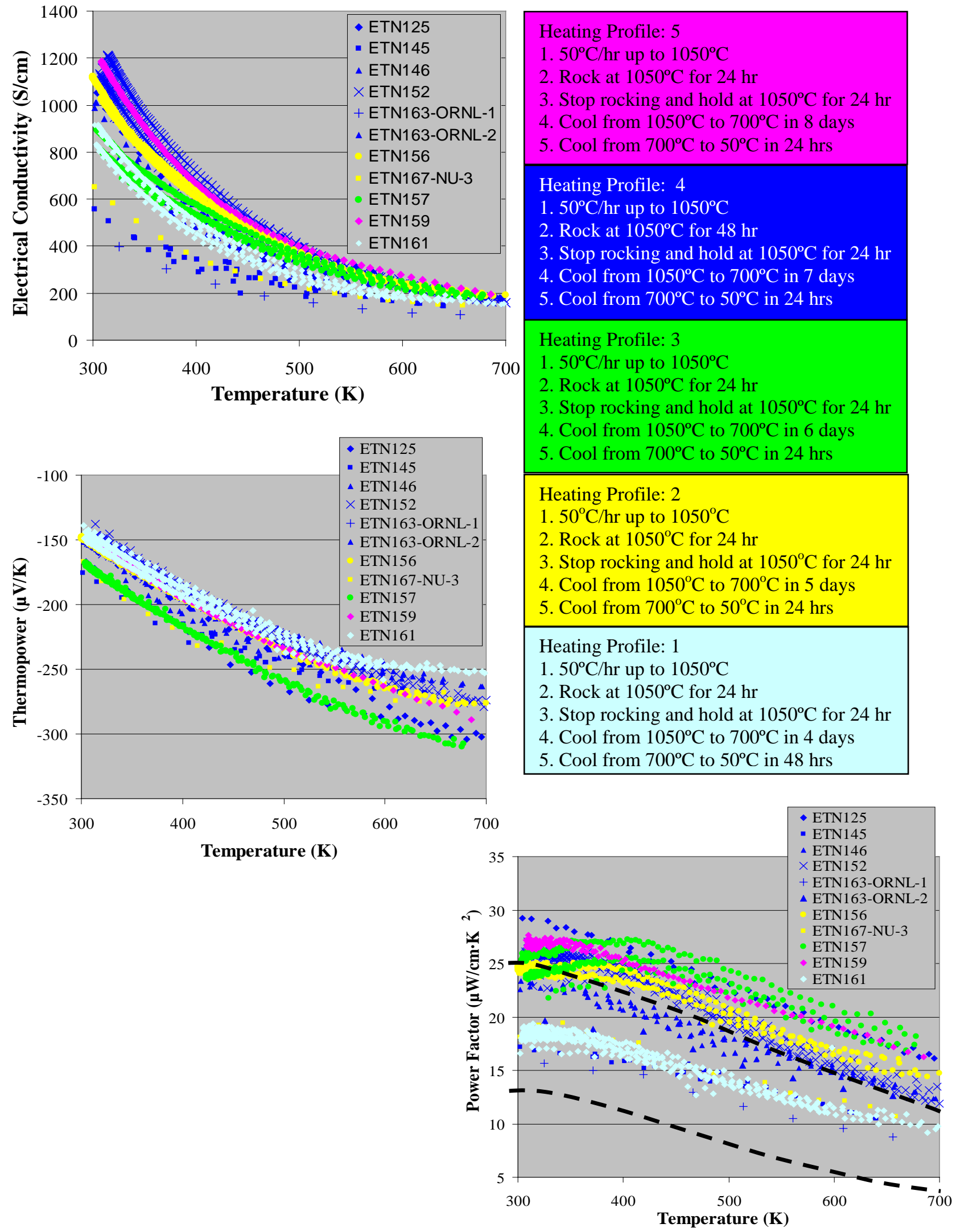

Figure 8.3. Transport data for $\mathrm{Ag}_{0.86} \mathrm{~Pb}_{19} \mathrm{Sb}_{1.0} \mathrm{Te}_{20}(m=19)$ samples.
Heating Profile: 5

1. $50^{\circ} \mathrm{C} / \mathrm{hr}$ up to $1050^{\circ} \mathrm{C}$

2. Rock at $1050^{\circ} \mathrm{C}$ for $24 \mathrm{hr}$

3. Stop rocking and hold at $1050^{\circ} \mathrm{C}$ for $24 \mathrm{hr}$

4. Cool from $1050^{\circ} \mathrm{C}$ to $700^{\circ} \mathrm{C}$ in 8 days

5. Cool from $700^{\circ} \mathrm{C}$ to $50^{\circ} \mathrm{C}$ in 24 hrs

\section{Heating Profile: 4}

1. $50^{\circ} \mathrm{C} / \mathrm{hr}$ up to $1050^{\circ} \mathrm{C}$

2. Rock at $1050^{\circ} \mathrm{C}$ for $48 \mathrm{hr}$

3. Stop rocking and hold at $1050^{\circ} \mathrm{C}$ for $24 \mathrm{hr}$

4. Cool from $1050^{\circ} \mathrm{C}$ to $700^{\circ} \mathrm{C}$ in 7 days

5. Cool from $700^{\circ} \mathrm{C}$ to $50^{\circ} \mathrm{C}$ in 24 hrs

Heating Profile: 3

1. $50^{\circ} \mathrm{C} / \mathrm{hr}$ up to $1050^{\circ} \mathrm{C}$

2. Rock at $1050^{\circ} \mathrm{C}$ for $24 \mathrm{hr}$

3. Stop rocking and hold at $1050^{\circ} \mathrm{C}$ for $24 \mathrm{hr}$

4. Cool from $1050^{\circ} \mathrm{C}$ to $700^{\circ} \mathrm{C}$ in 6 days

5. Cool from $700^{\circ} \mathrm{C}$ to $50^{\circ} \mathrm{C}$ in $24 \mathrm{hrs}$

Heating Profile: 2

1. $50^{\circ} \mathrm{C} / \mathrm{hr}$ up to $1050^{\circ} \mathrm{C}$

2. Rock at $1050^{\circ} \mathrm{C}$ for $24 \mathrm{hr}$

3. Stop rocking and hold at $1050^{\circ} \mathrm{C}$ for $24 \mathrm{hr}$

4. Cool from $1050^{\circ} \mathrm{C}$ to $700^{\circ} \mathrm{C}$ in 5 days

5. Cool from $700^{\circ} \mathrm{C}$ to $50^{\circ} \mathrm{C}$ in $24 \mathrm{hrs}$

Heating Profile: 1

1. $50^{\circ} \mathrm{C} / \mathrm{hr}$ up to $1050^{\circ} \mathrm{C}$

2. Rock at $1050^{\circ} \mathrm{C}$ for $24 \mathrm{hr}$

3. Stop rocking and hold at $1050^{\circ} \mathrm{C}$ for $24 \mathrm{hr}$

4. Cool from $1050^{\circ} \mathrm{C}$ to $700^{\circ} \mathrm{C}$ in 4 days

5. Cool from $700^{\circ} \mathrm{C}$ to $50^{\circ} \mathrm{C}$ in 48 hrs 

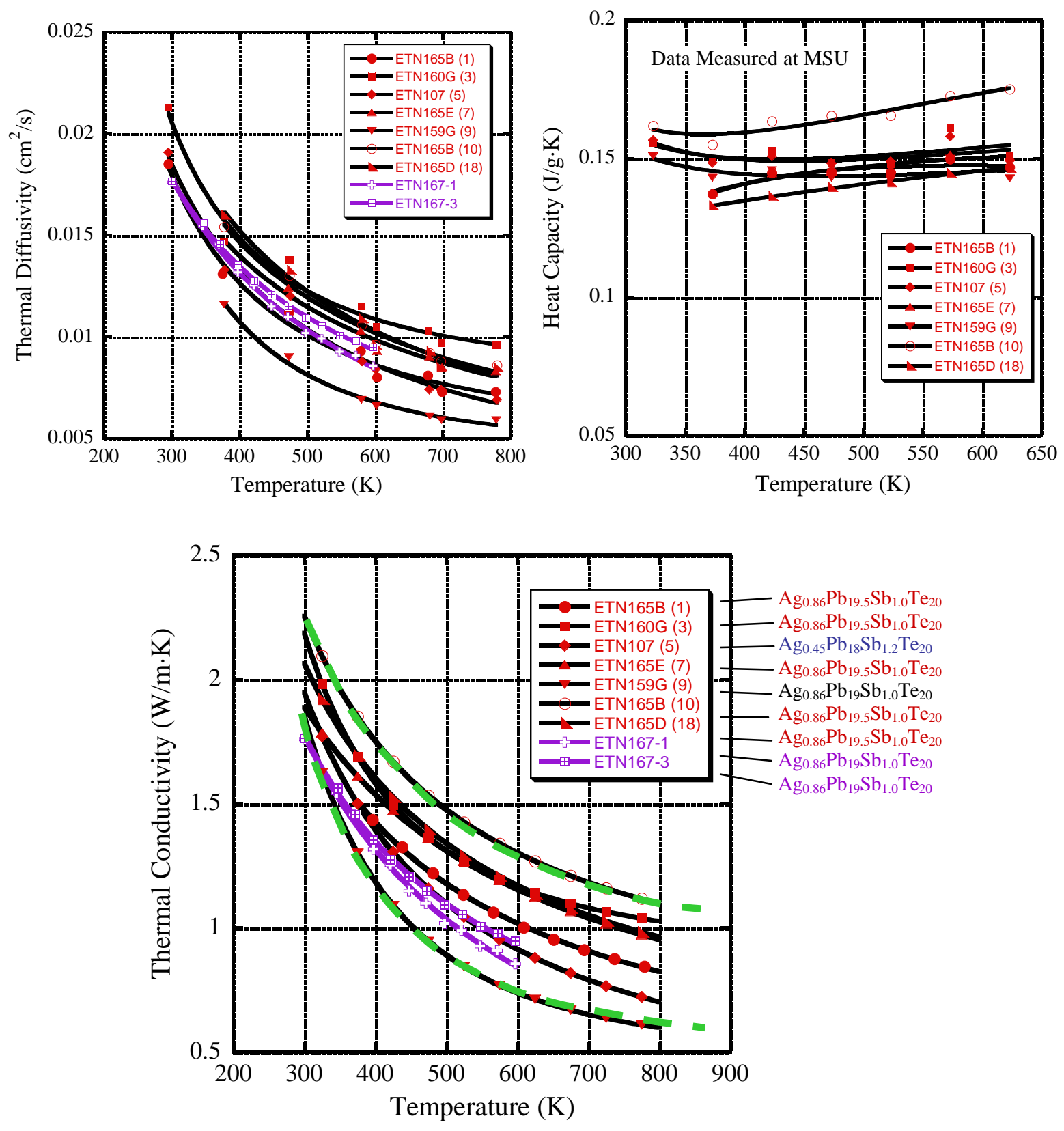

Figure 8.4. Thermal diffusivity, heat capacity and thermal conductivity for $\mathrm{Ag}_{0.86} \mathrm{~Pb}_{19} \mathrm{Sb}_{1.0} \mathrm{Te}_{20}(m=19)$ samples. 

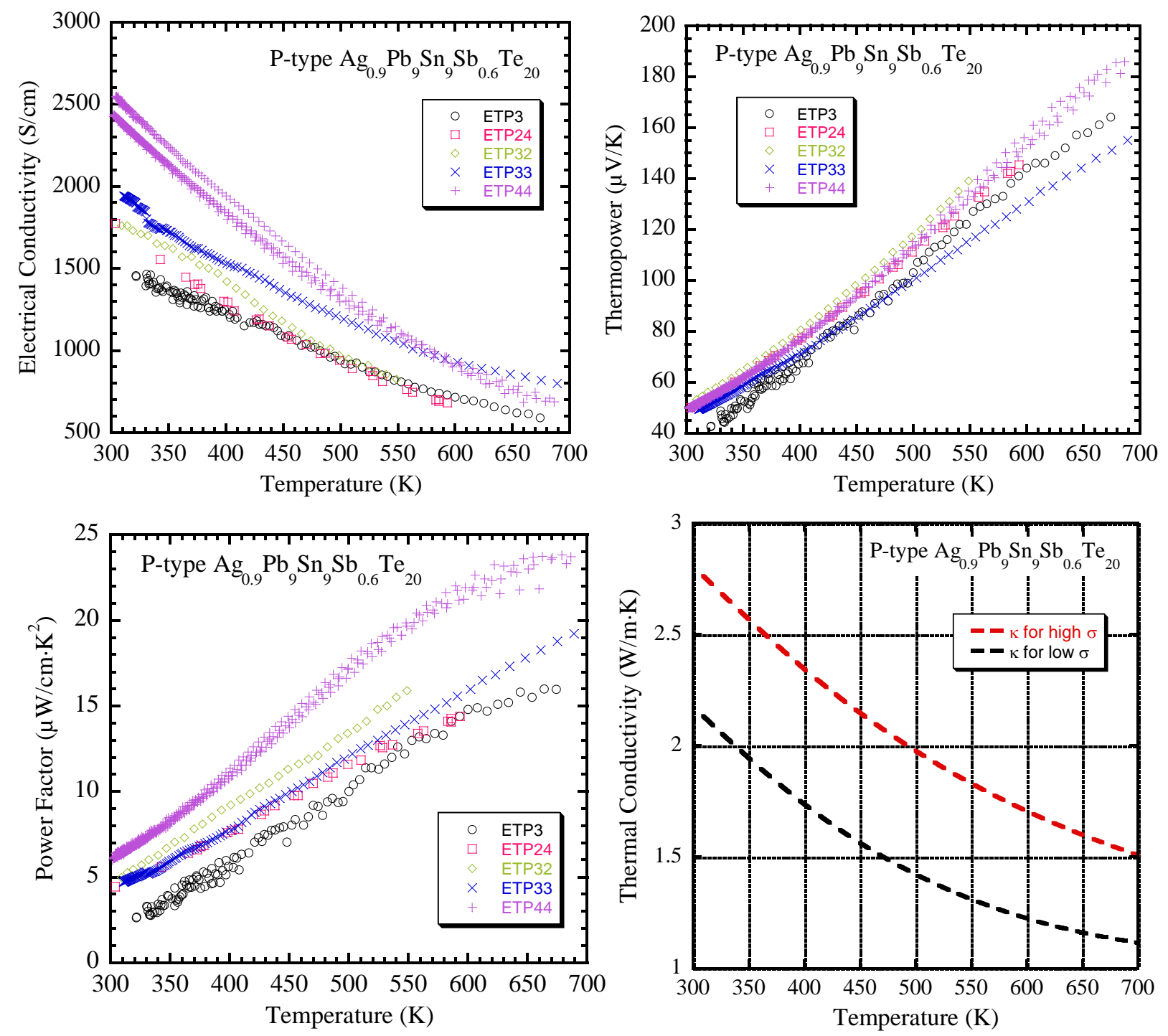

Figure 8.5. Compilation plot of transport data for $\mathrm{Ag}_{0.9} \mathrm{~Pb}_{9} \mathrm{Sn}_{9} \mathrm{Sb}_{0.6} \mathrm{Te}_{20}$ cast samples.

The samples used for transport measurements are the same dimensions as the legs used in the modules ( $5 \mathrm{~mm} \times 5 \mathrm{~mm} \times 7 \mathrm{~mm}$ ), while thermal diffusivity samples must be thin plate structures of approximately $12 \mathrm{~mm} \times 12 \mathrm{~mm} \times 1.5 \mathrm{~mm}$. While samples can be used from the same ingot, they are separate parts of the ingot; therefore, we utilize the maximum and minimum values for the data sets of power factor and thermal conductivity in calculating the range of ZT values for these materials. Based on the range of values outlined by dashed lines in Figure 8.3 and Figure 8.4, the corresponding range of ZT values was calculated and is shown in Figure 8.6. 

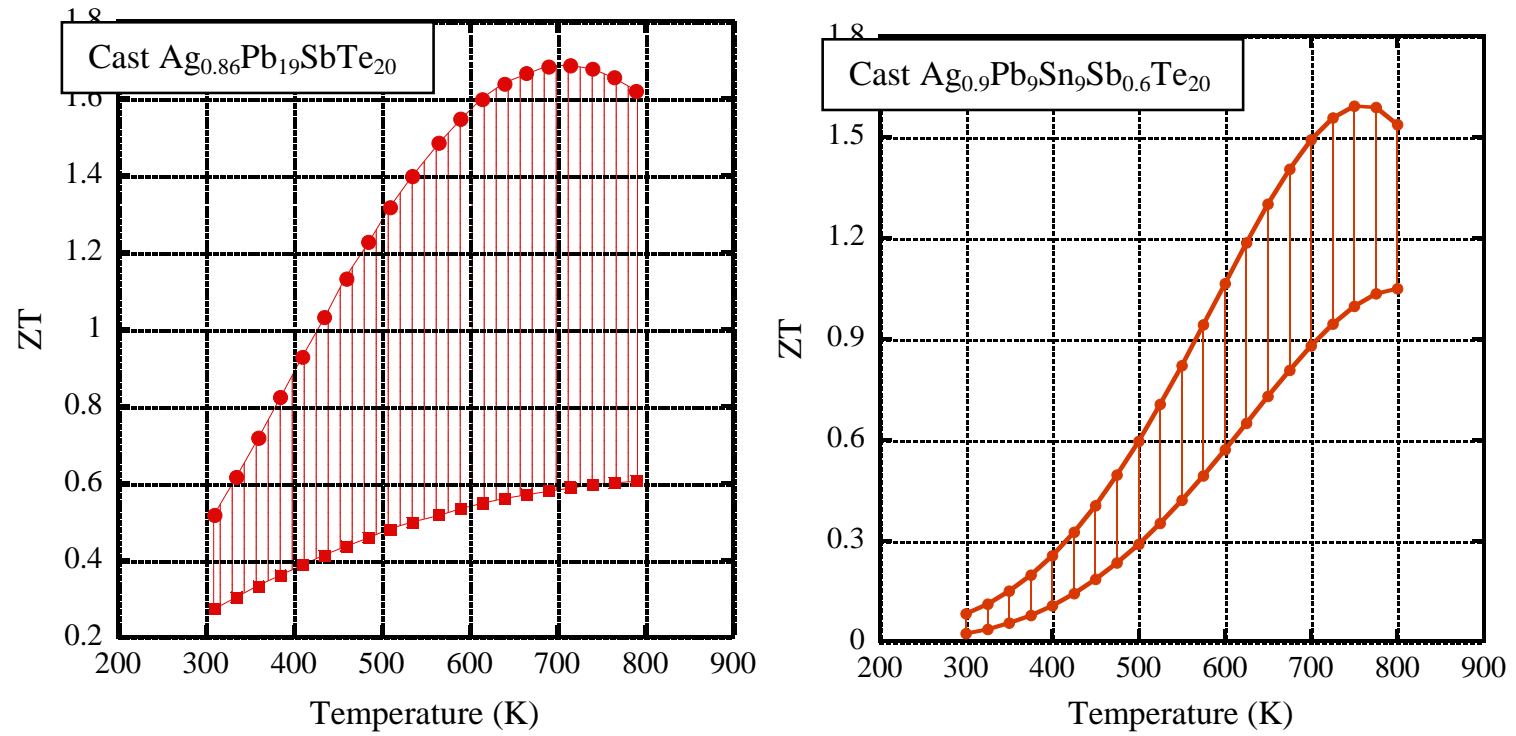

Figure 8.6. Range of $\mathrm{ZT}$ values for $\mathrm{LAST}\left(\mathrm{Ag}_{0.86} \mathrm{~Pb}_{19} \mathrm{SbTe}_{20}\right)$ and LASTT $\left(\mathrm{Ag}_{0.9} \mathrm{~Pb}_{9} \mathrm{Sn}_{9} \mathrm{Sb}_{0.6} \mathrm{Te}_{20}\right)$ cast samples based on the data in Figures 8.3 through 8.5.

Oxidization and loss of material through volatilization can occur at high temperatures. To prevent these effects, several coating materials have been investigated. A high temperature chemical set adhesive was found to show negligible weight change for N-type and P-type samples enclosed in the adhesive and held at $600^{\circ} \mathrm{C}$ for 8 hours.
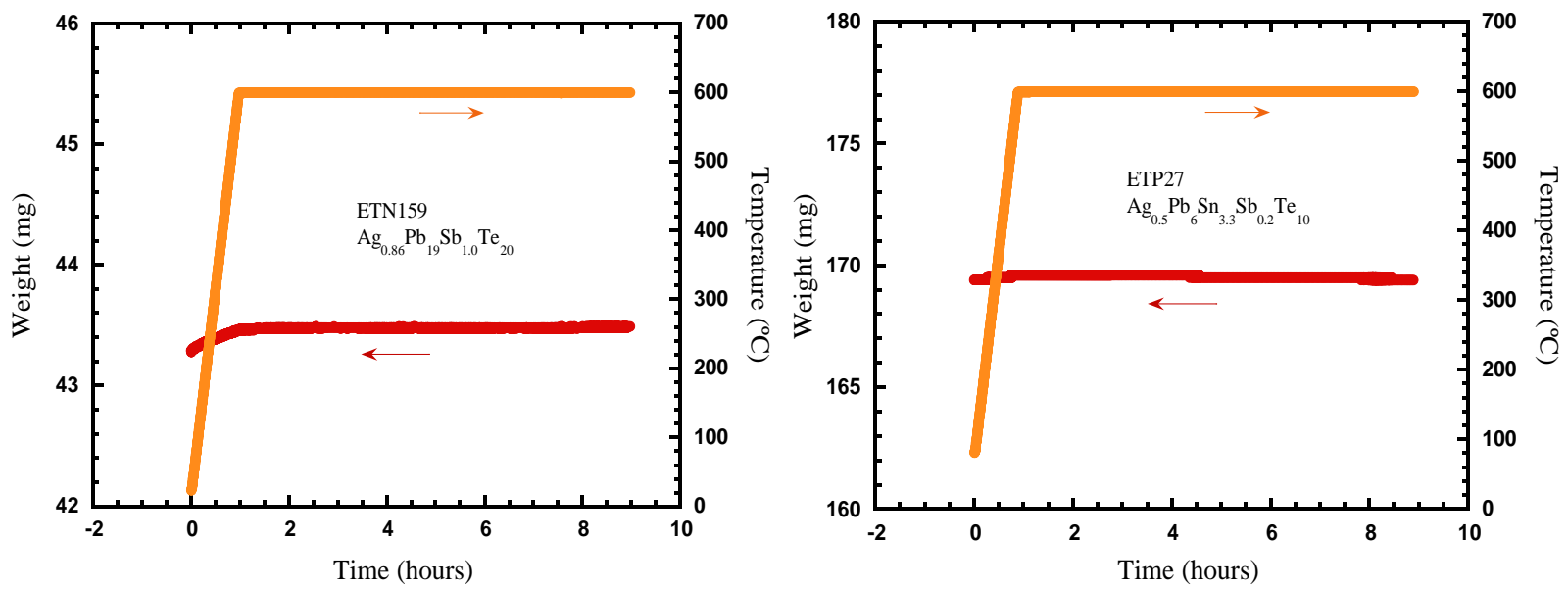

Figure 8.7. TGA results of encapsulation of $\mathrm{N}$-type $\mathrm{Ag}_{0.86} \mathrm{~Pb}_{19} \mathrm{Sb}_{1.0} \mathrm{Te}_{20}$ and P-type $\mathrm{Ag}_{0.5} \mathrm{~Pb}_{6} \mathrm{Sn}_{3.3} \mathrm{Sb}_{0.2} \mathrm{Te}_{10}$ by a high temperature chemical set adhesive. As shown by the red lines, negligible weight change occurred over an 8 hour period.

\subsection{Hot Pressed Materials}

Significant advancements in the hot pressing of these materials have been made and several samples have been measured. These samples show changes as a function of temperature cycling. 
Bloating of the samples has been seen after exposure to high temperatures in some of these initial samples.

Figures 8.8 and 8.9 show transport measurements on one of the initial hot pressed N-type samples. Figure 8.8 shows high temperature data while cycling the temperature 13 times. Each new cycle showed an increase in the electrical conductivity of the sample. The thermopower decreased in magnitude during the first few cycles and then repeated after cycle 6 . The electrical conductivity of these hot pressed samples shows a different trend as a function of temperature from the cast samples. An activated behavior typically seen in semiconductors is found. However, it peaks at approximately $500 \mathrm{~K}$ and decreases as the temperature increases for temperatures greater than $500 \mathrm{~K}$. The same sample was then measured to low temperatures, (shown in Figure 8.9) and the temperature cycled nine times. The electrical conductivity continued to increase over these cycles. However, the thermopower shows good repeatability over the temperature cycles. The thermal conductivity also showed good repeatability with $2.2(\mathrm{~W} / \mathrm{m} \cdot \mathrm{K})$ at $100 \mathrm{~K}$, falling to $1.2(\mathrm{~W} / \mathrm{m} \cdot \mathrm{K})$ at $400 \mathrm{~K}$. The figure of merit rapidly increases as a function of temperature to a value of 0.3 at $400 \mathrm{~K}$.
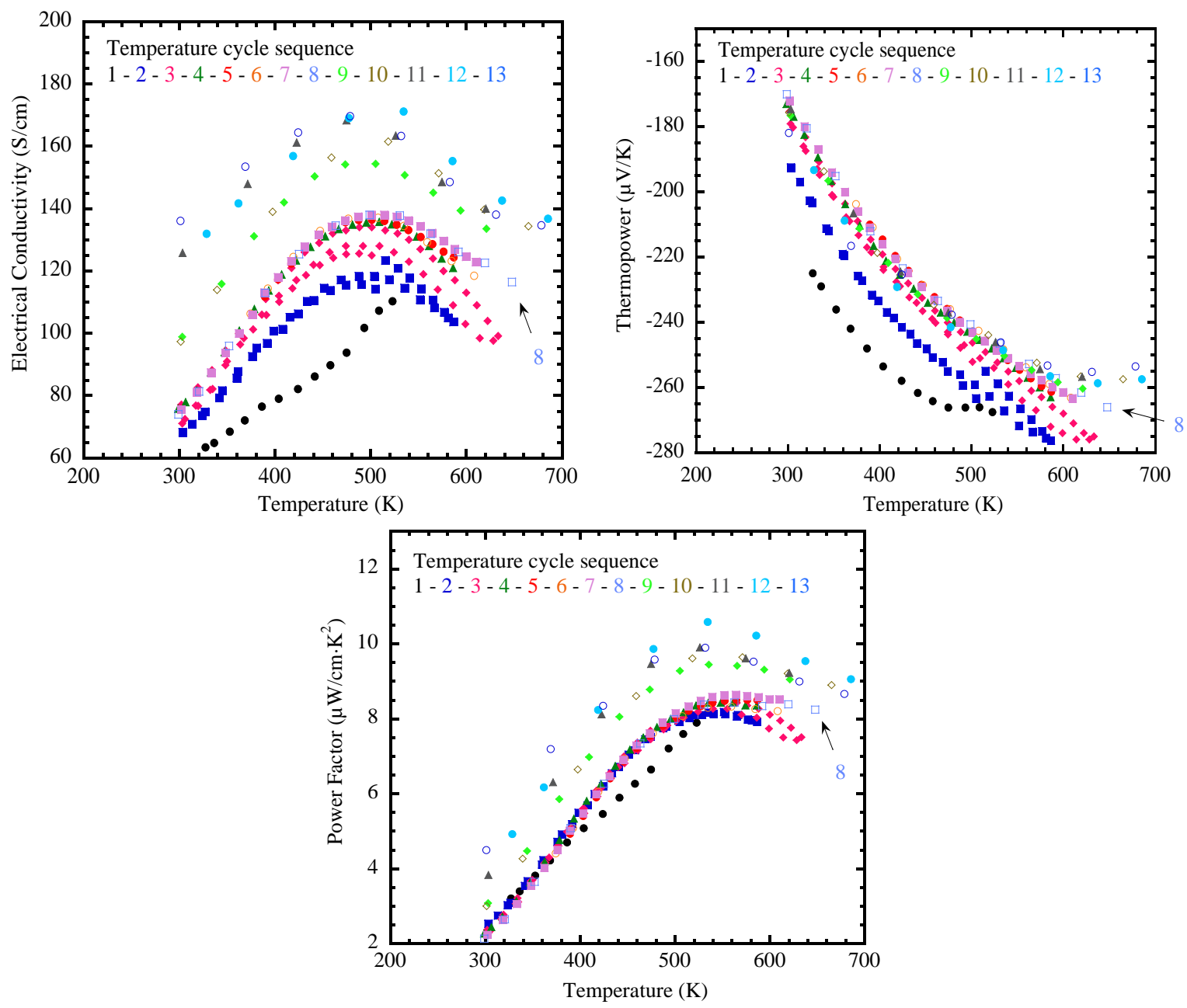

Figure 8.8. Electrical conductivity, thermopower and power factor for hot pressed N-type samples. Data were taken over thirteen temperature cycles. 

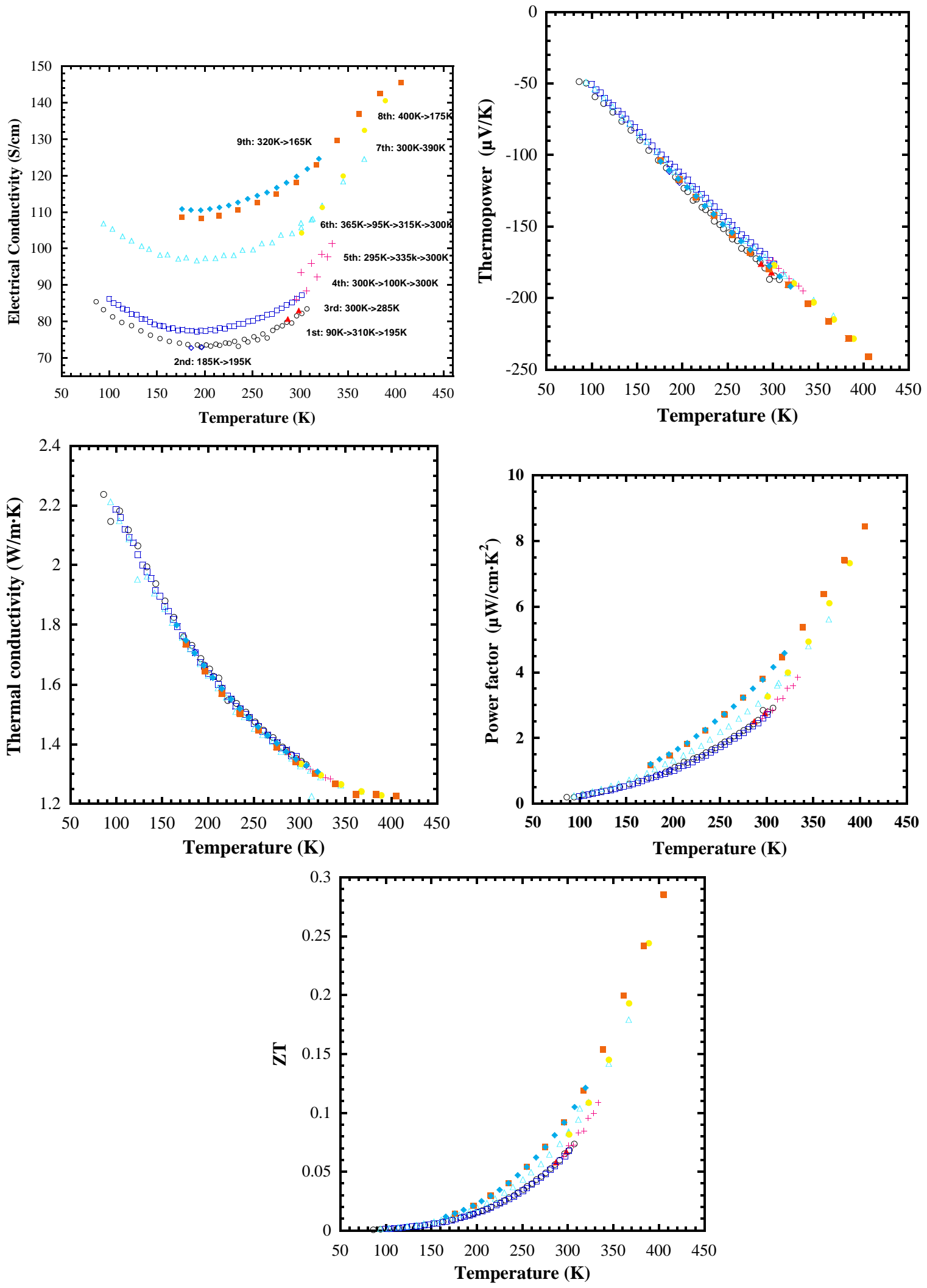

Figure 8.9. Low temperature measurements on the same sample shown in Figure 8.8 after the high temperature measurements. 
Hot pressed P-type samples showed excellent transport properties as compared to cast samples. Temperature cycling showed good repeatability up to $600 \mathrm{~K}$. Some bloating of the sample could be seen after the temperature cycles (Figure 8.10) were completed. Thermal conductivity measurements were also taken on another sample from this same hot pressed puck and the figure of merit, ZT, exceeds 0.8 above $600 \mathrm{~K}$.
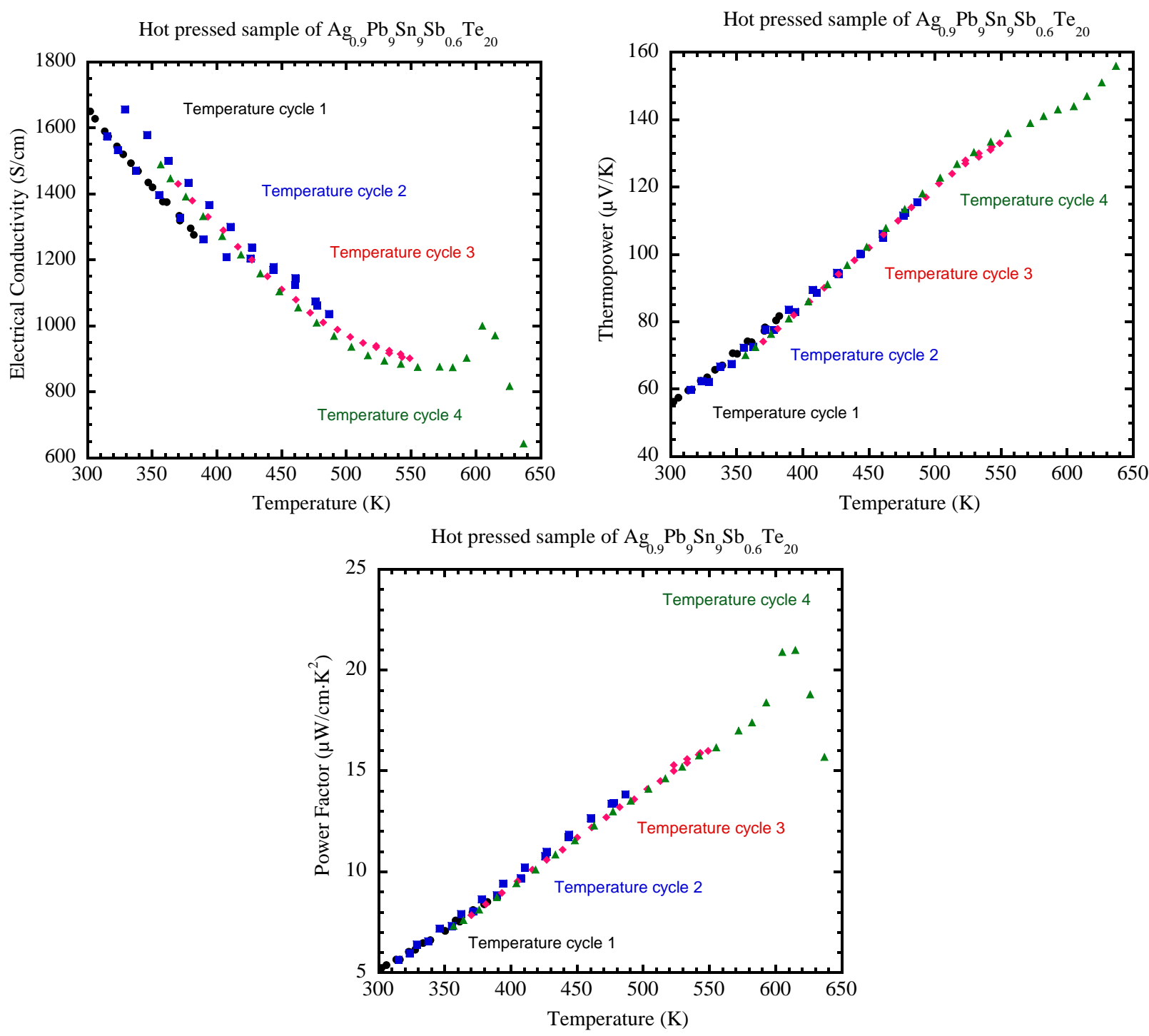

Figure 8.10. Hot pressed P-type $\mathrm{Ag}_{0.9} \mathrm{~Pb}_{9} \mathrm{Sn}_{9} \mathrm{Sb}_{0.6} \mathrm{Te}_{20}$ sample measured through four temperature cycles.

Utilizing the thermal conductivity of the cast materials that were measured at ORNL and at Northwestern, the above power factors give ZT values as shown below in Figure 8.11, on the right. Higher lead content hot pressed samples ((Figure 8.12) show the following properties and 
show less chipping and less cracking during cutting processes. Lead content of 19.5 for cast samples have also been investigated and are shown in Figure 8.13.

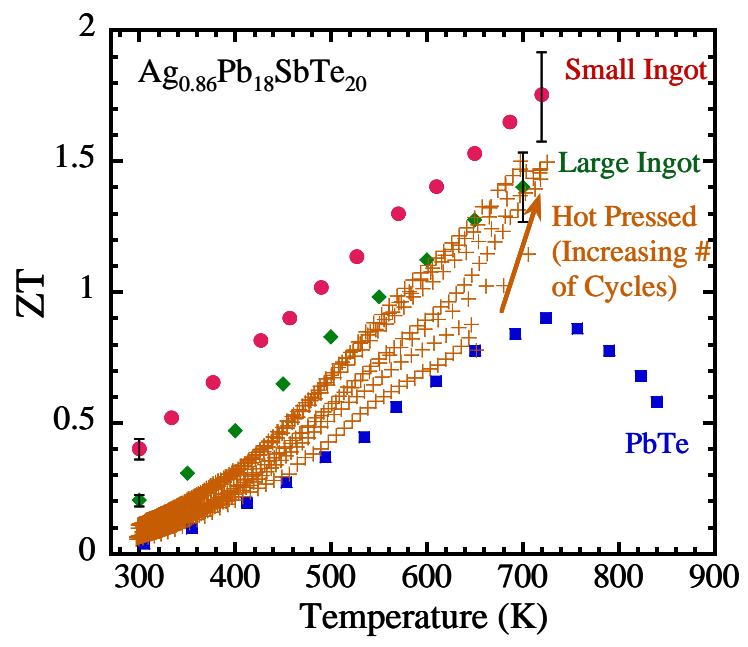

Figure 8.11. Comparison of ZT values for LAST cast, and hot pressed samples along with PbTe. 

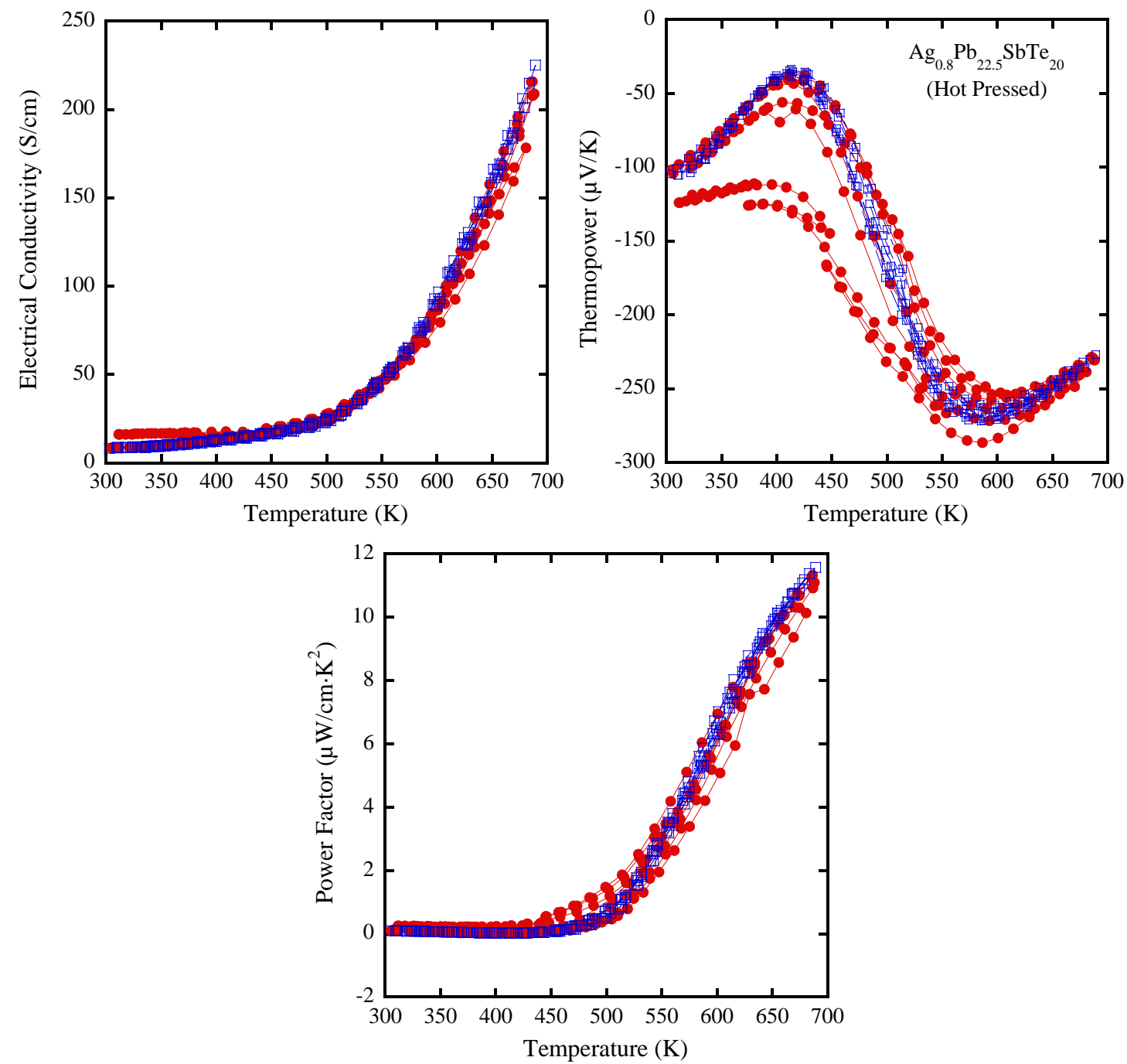

Figure 8.12. Electrical conductivity, thermopower and the corresponding power factor for higher lead content hot pressed LAST samples $\left(\mathrm{Ag}_{0.8} \mathrm{~Pb}_{22.5} \mathrm{SbTe}_{20}\right)$. 

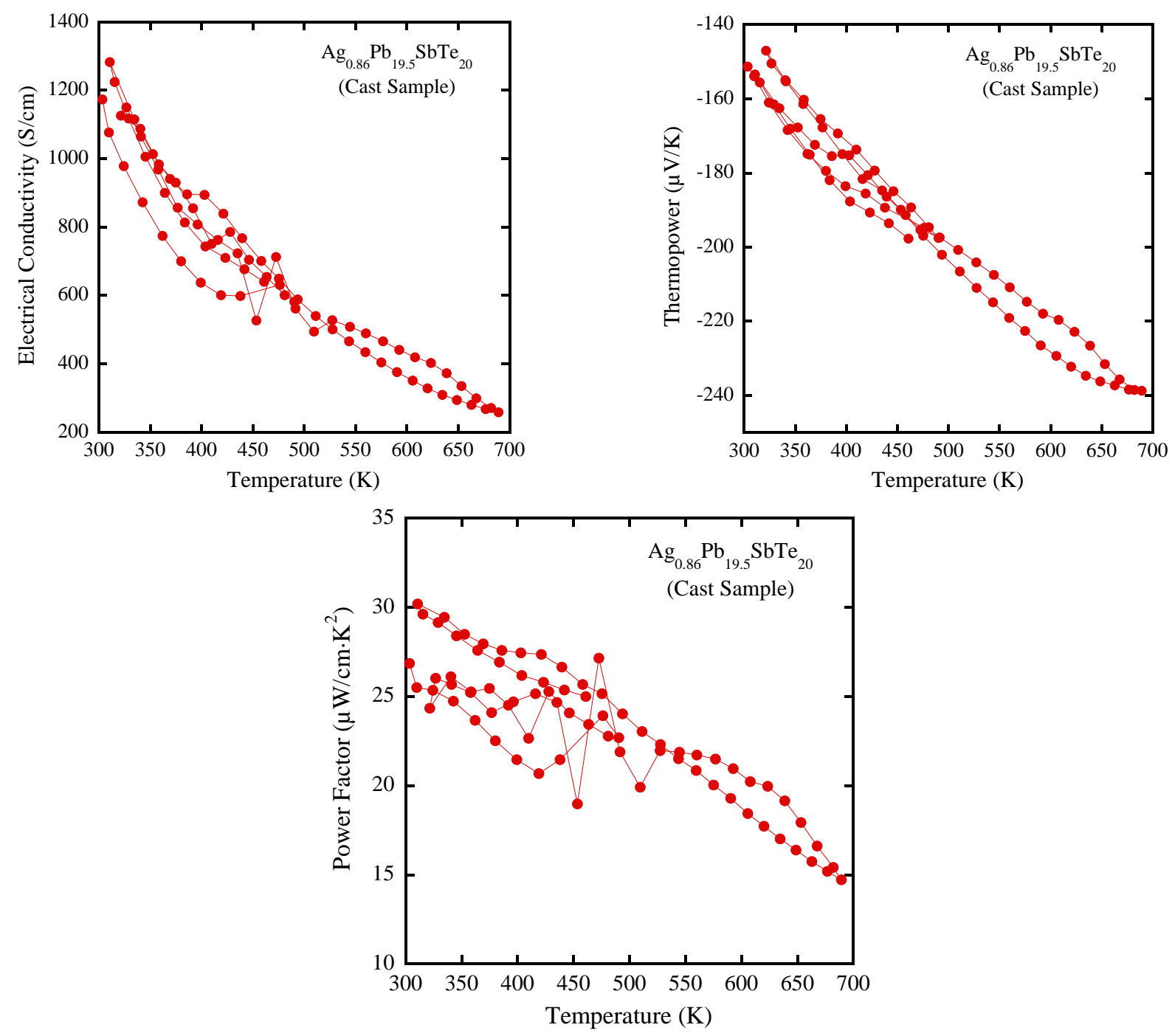

Figure 8.13. Electrical conductivity, thermopower and the corresponding power factor for higher lead content cast LAST samples $\left(\mathrm{Ag}_{0.86} \mathrm{~Pb}_{19.5} \mathrm{SbTe}_{20}\right)$.

\subsection{Module Fabrication with LAST and LAST Materials}

Diffusion bonded contacts between stainless steel and cast samples have shown contact resistivity's below $20\left(\mu \Omega \cdot \mathrm{cm}^{2}\right)$. Similarly, diffusion bonding between hot pressed samples and stainless steel also show low contact resistances (shown in Figure 8.14) after a $700{ }^{\circ} \mathrm{C}$ bonding procedure. 


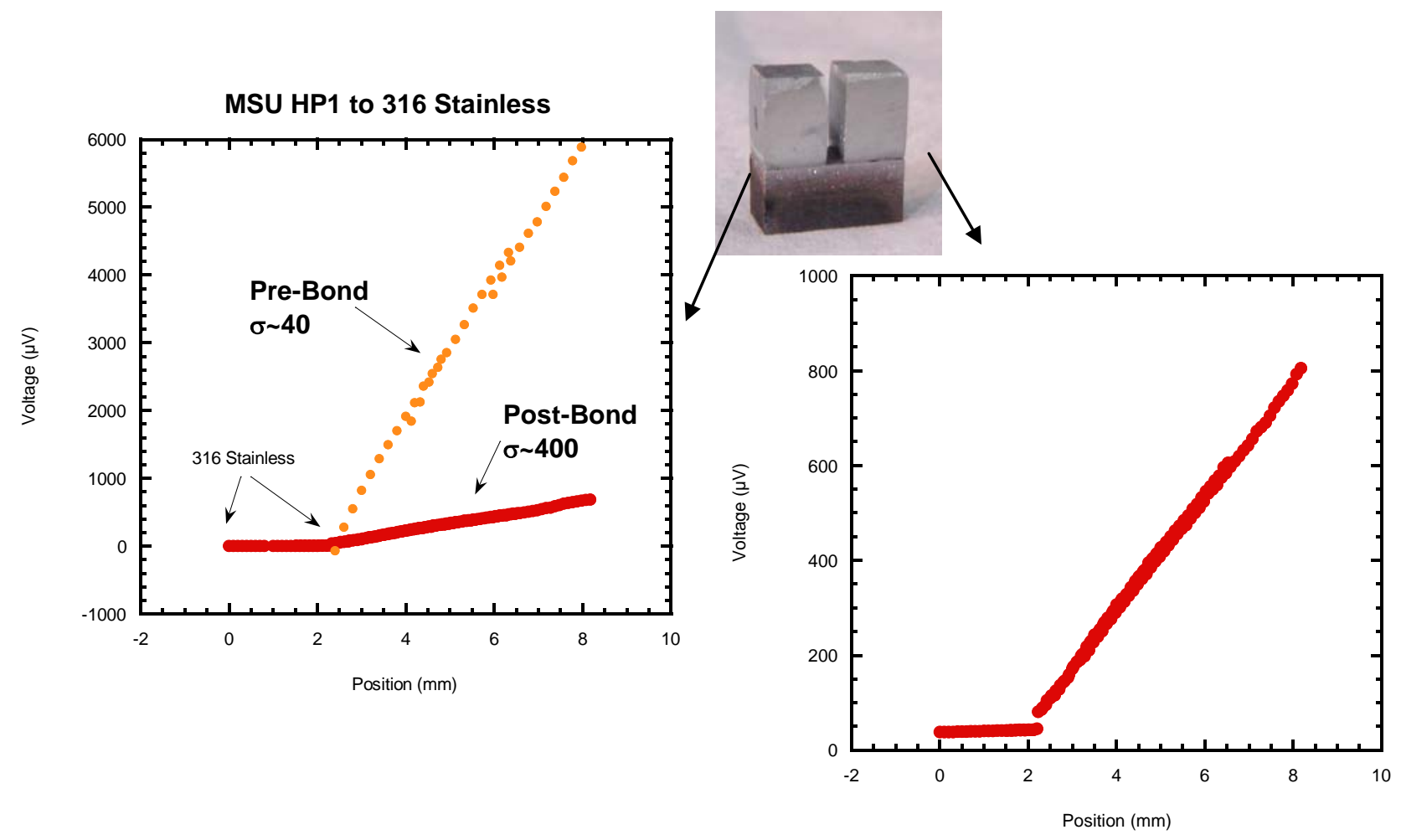

Figure 8.14. Two N-type legs of hot pressed $\mathrm{Ag}_{0.86} \mathrm{~Pb}_{19} \mathrm{Sb}_{1.0} \mathrm{Te}_{20}$ diffusion bonded to 316 stainless steel.

Similarly, hot pressed P-type samples of $\mathrm{Ag}_{0.9} \mathrm{~Pb}_{9} \mathrm{Sn}_{9} \mathrm{Sb}_{0.6} \mathrm{Te}_{20}$ which are bonded to stainless steel also show low contact resistivity of less than $20\left(\mu \Omega \cdot \mathrm{cm}^{2}\right)$ as shown in Figure 8.15.

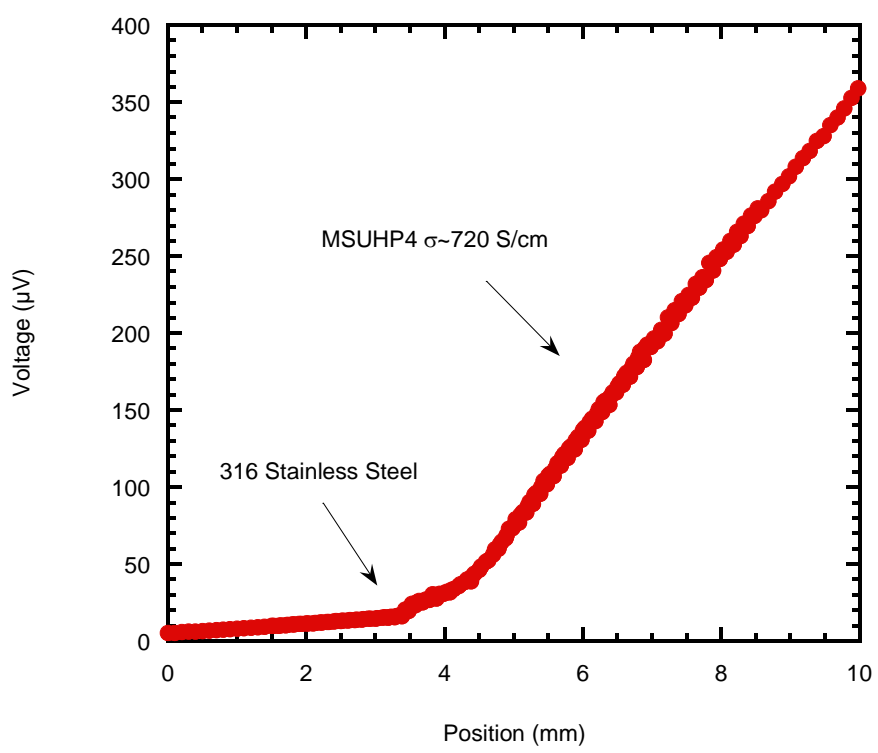

Figure 8.15. A P-type leg of hot pressed $\mathrm{Ag}_{0.9} \mathrm{~Pb}_{9} \mathrm{Sn}_{9} \mathrm{Sb}_{0.6} \mathrm{Te}_{20}$ diffusion bonded to 316 stainless steel. 
At temperatures below $\left(450 \mathrm{~K}\right.$ ), the figure of merit is less than that of $\mathrm{Bi}_{2} \mathrm{Te}_{3}$ based materials and has good compatibility. Therefore, segmented legs of $\mathrm{N}$-type $\mathrm{Bi}_{2} \mathrm{Te}_{3}$ and $\mathrm{Ag}_{0.86} \mathrm{~Pb}_{19} \mathrm{Sb}_{1.0} \mathrm{Te}_{20}$ and P-type $\mathrm{Bi}_{2} \mathrm{Te}_{3}$ and $\mathrm{Ag}_{0.9} \mathrm{~Pb}_{9} \mathrm{Sn}_{9} \mathrm{Sb}_{0.6} \mathrm{Te}_{20}$ have been investigated (Figure 8.16).

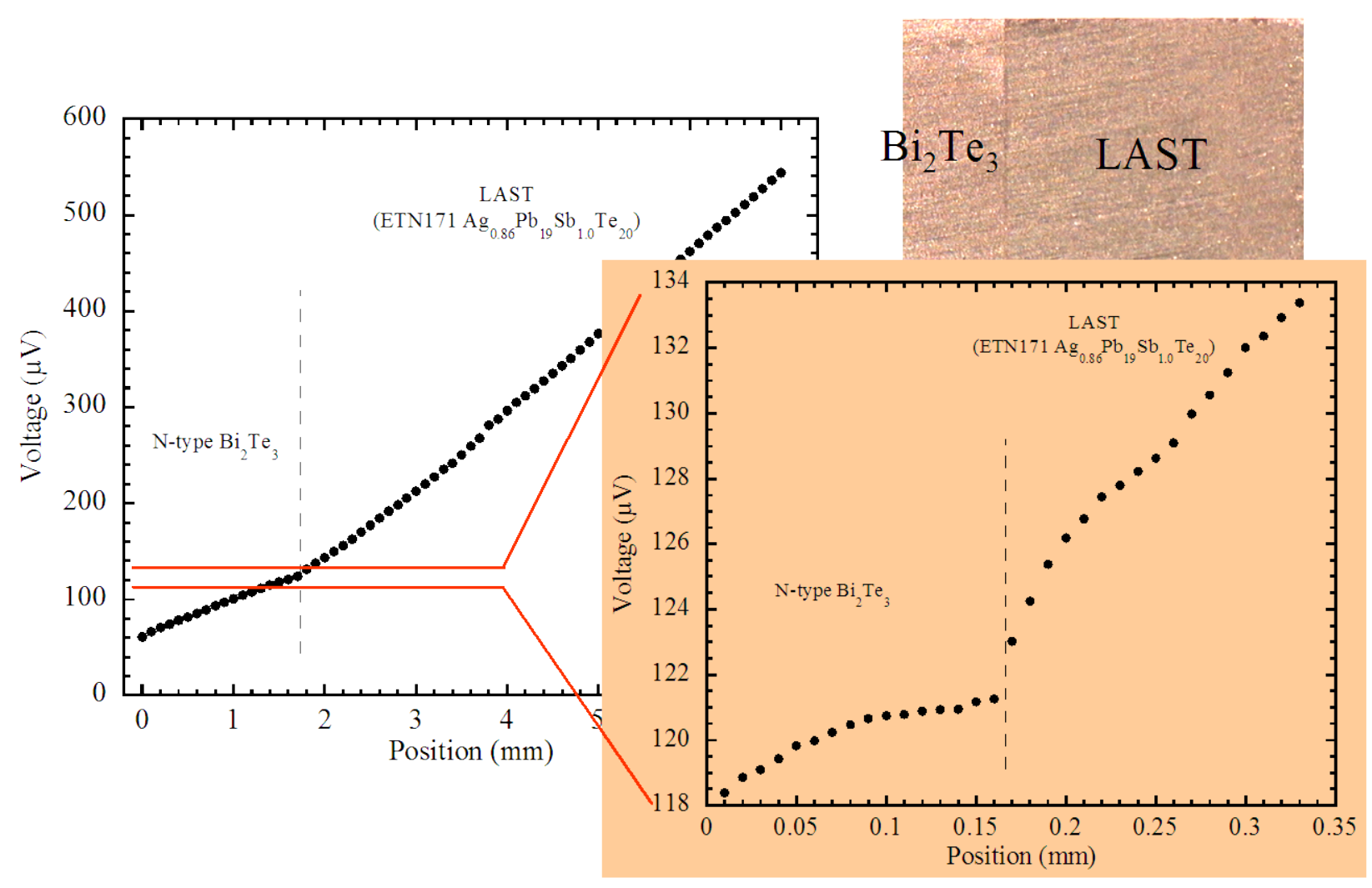

$\mathrm{Bi}_{2} \mathrm{Te}_{3}$ Material supplied by Tellurex Corporation.

Figure 8.16. Measurement of the contact resistivity for the soldered junction between $\mathrm{N}$-type $\mathrm{Bi}_{2} \mathrm{Te}_{3}$ and $\mathrm{Ag}_{0.86} \mathrm{~Pb}_{19} \mathrm{Sb}_{1.0} \mathrm{Te}_{20}$. 
Similarly, for P-type samples, segmented legs have been fabricated with very low contact resistivity between the $\mathrm{Bi}_{2} \mathrm{Te}_{3}$ and $\mathrm{Ag}_{0.9} \mathrm{~Pb}_{9} \mathrm{Sn}_{9} \mathrm{Sb}_{0.6} \mathrm{Te}_{20}$ (Figure 8.17).

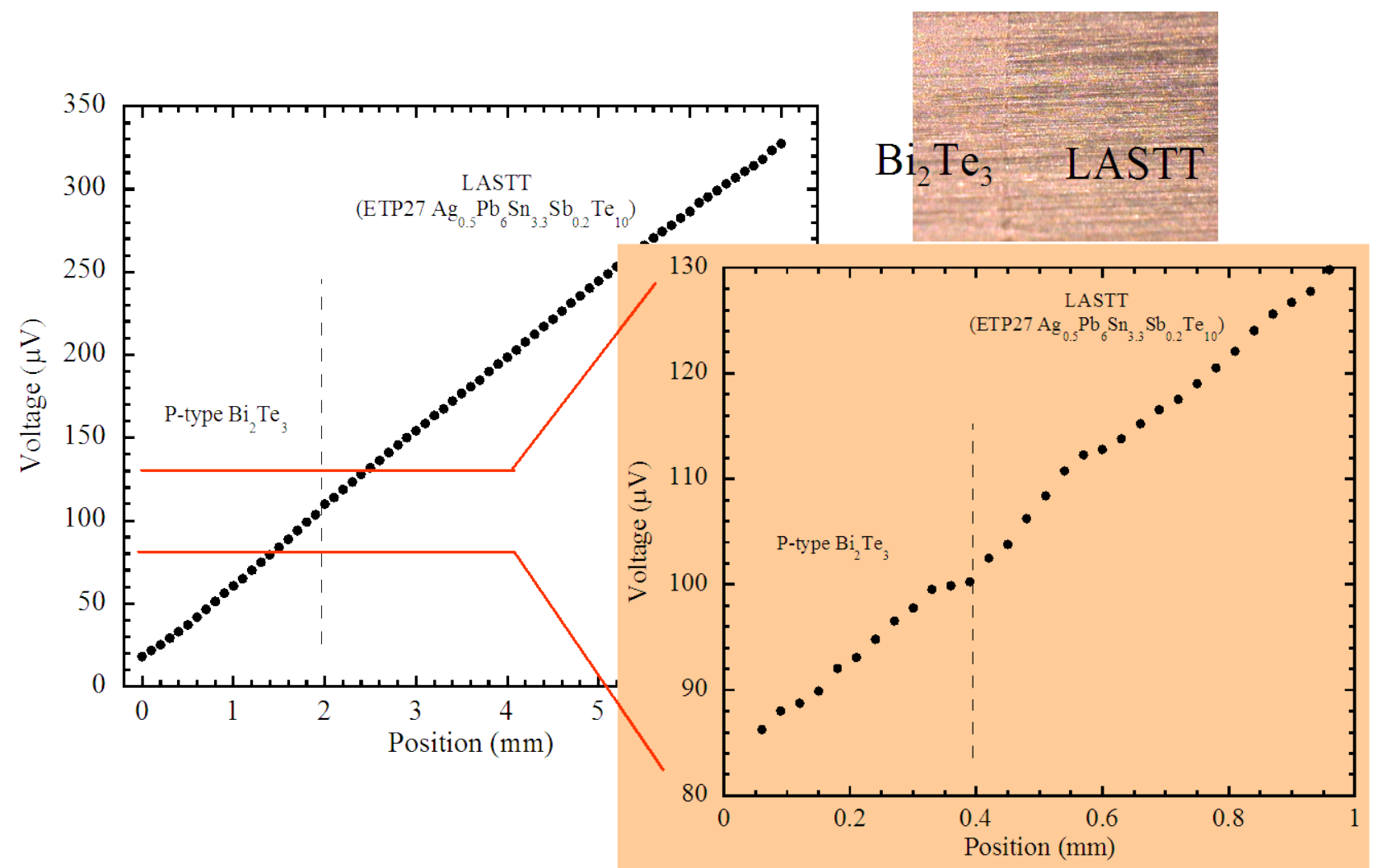

$\mathrm{Bi}_{2} \mathrm{Te}_{3}$ Material supplied by Tellurex Corporation.

Figure 8.17. Measurement of the contact resistivity

for the soldered junction between P-type $\mathrm{Bi}_{2} \mathrm{Te}_{3}$ and $\mathrm{Ag}_{0.9} \mathrm{~Pb}_{9} \mathrm{Sn}_{9} \mathrm{Sb}_{0.6} \mathrm{Te}_{20}$. 
Diffusion bonds to N-type LAST and P-type LASTT have been made and measured by scanning voltage probe measurements (Figure 8.18). P-type samples continually show high electrical conductivity above $1,000(\mathrm{~S} / \mathrm{cm})$ and low contact resistance. However, some non-uniformities can be seen in the data along the leg. The N-type samples also show good low contact resistances. However, the $\mathrm{N}$-type materials are three to four times more resistive than the P-type materials.
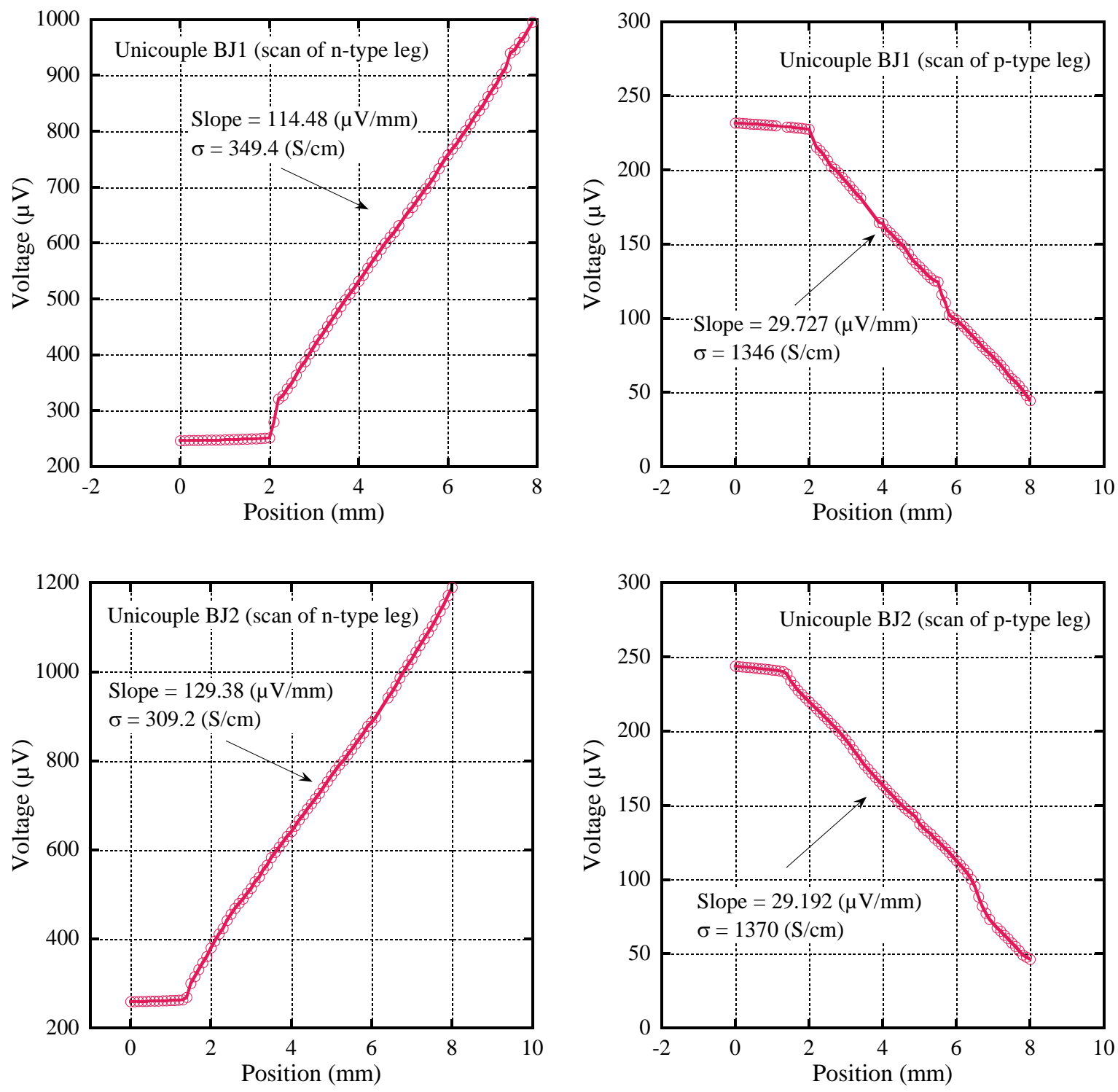

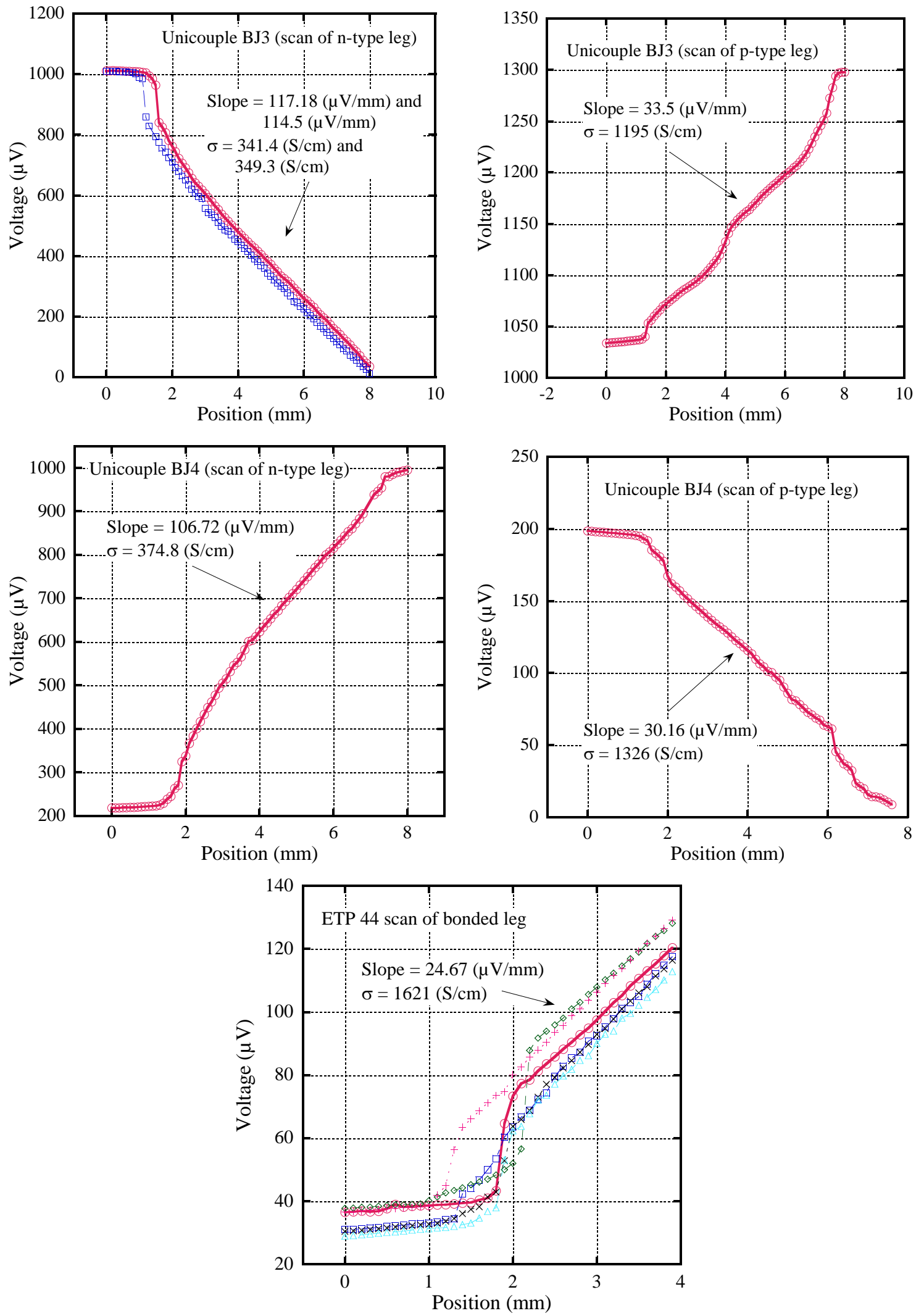

Figure 8.18. Contact resistance studies showing voltage scans across the N-type and P-type legs of couples. 
Modifications to the diffusion bonding structure, which includes a spring loaded design for constant mechanical pressure during the bonding process, has helped to improve repeatability of the low contact resistance junctions (Figure 8.19). The spring loaded structure is shown in Figure 8.20.

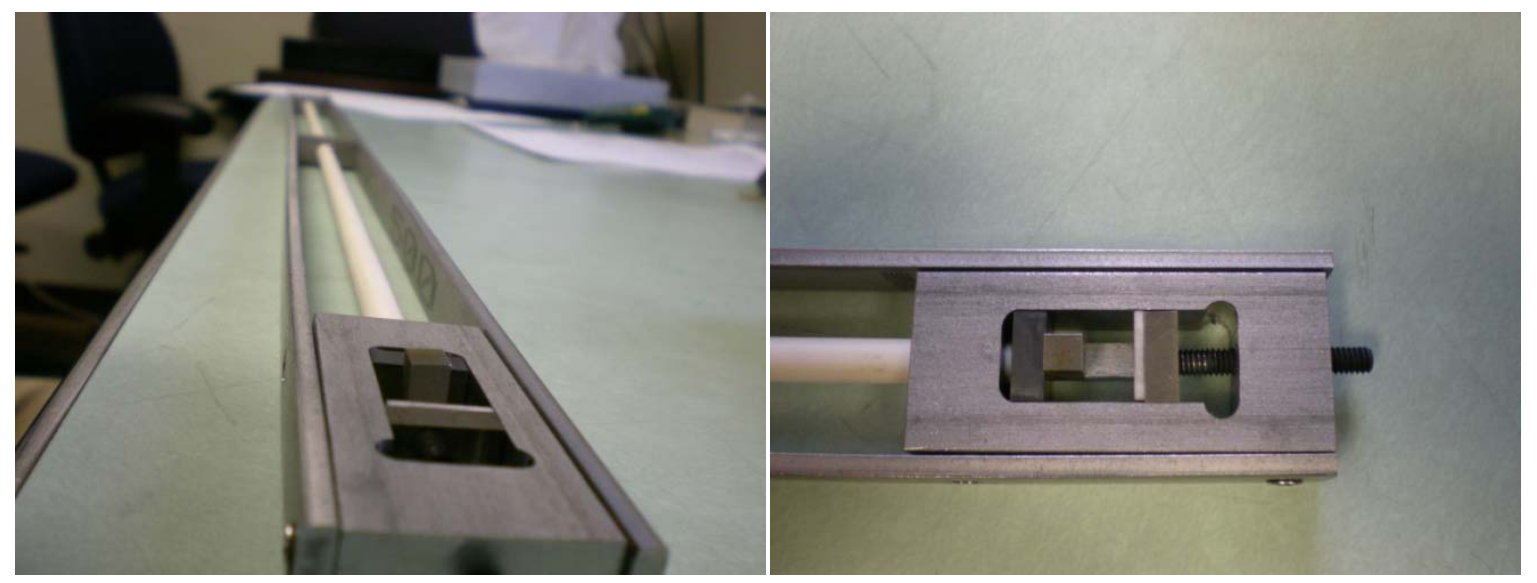

Figure 8.19. Spring loaded structure for constant mechanical pressure during the diffusion bonding process.

A procedure for fabricating metalized aluminum nitride substrates has been established. This utilizes a physical deposition system in the MSU, Electrical and Computer Engineering Department Cleanroom. A two step process is used [10], where a chromium deposition is followed by an oxidation process to convert the chromium to chromium oxide and bond it to the AlN substrate. The second deposition of chromium can then be electroplated or directly soldered to nickel and/or solder interconnects for the cold side electrodes. A 36-leg module substrate fabricated through this process is shown in Figure 8.20.

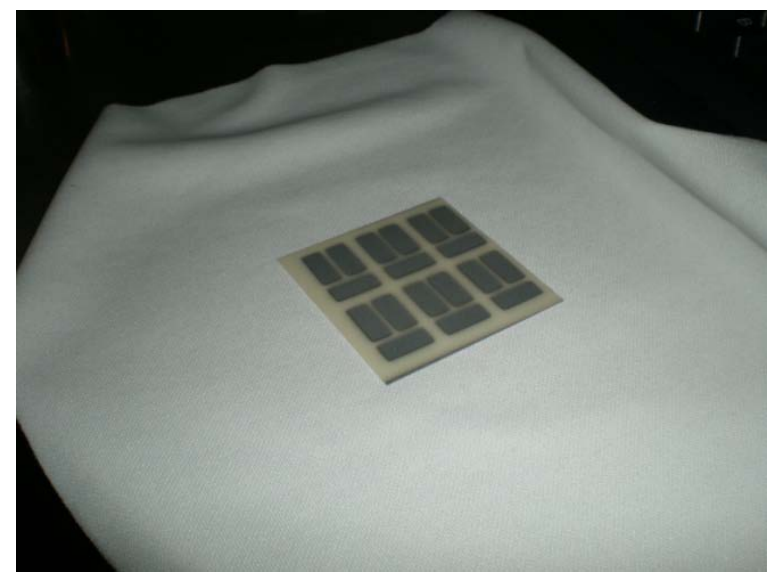

Figure 8.20. Metalized AlN substrate used for the cold side interconnects of a 36-leg module (or 18 couples). 
A long term module testing system has been assembled and used for monitoring of the output short circuit electrical current, open circuit voltage and hot and cold side temperatures for modules, while maintaining a background gas of argon at approximately one atmosphere of pressure (Figure 8.21).
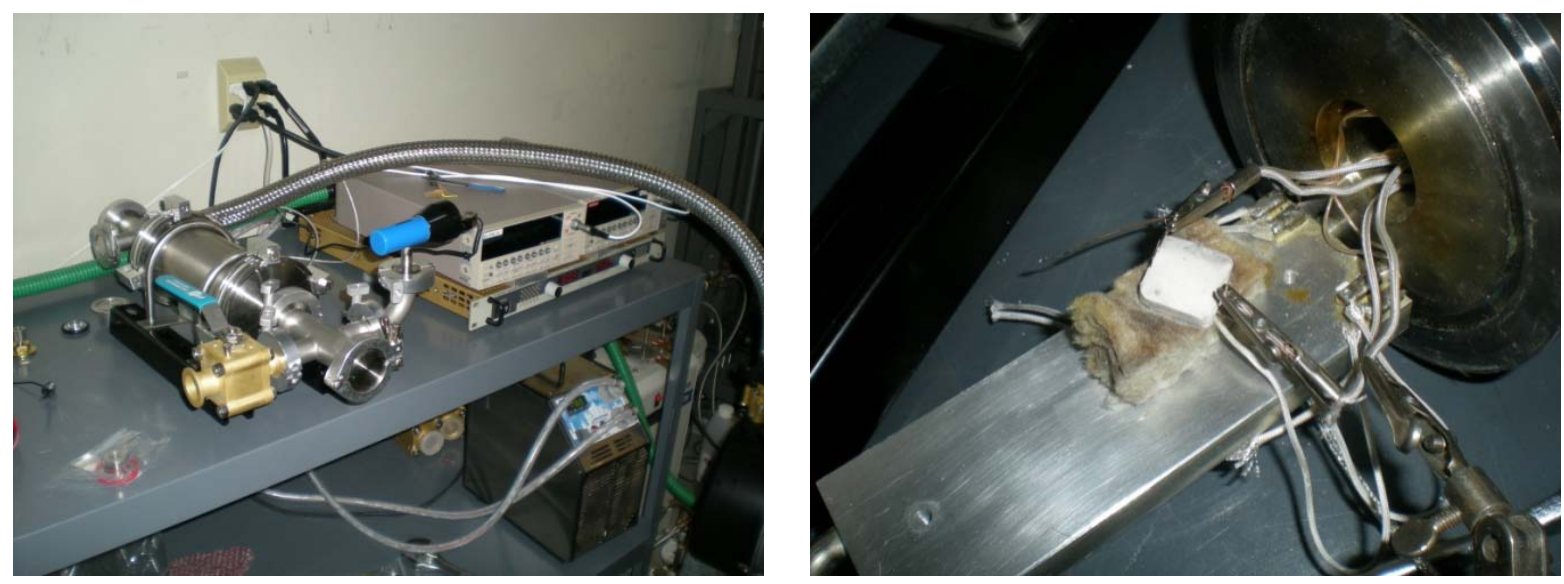

Figure 8.21. Long term module measurement system.

This measurement chamber for the system is relatively compact with vacuum valves included. Several chambers can be prepared with a single pumping station and periodically monitored by the same measurement equipment. Small heaters that can go to $>600{ }^{\circ} \mathrm{C}$ were difficult to locate in the marketplace. Cartridge heaters have been used in the past. However, their lifetime was found to be unacceptably short, therefore, we have established a method for encapsulating a nichrome (nickel chromium alloy) heater wire into a high temperature adhesive. An example heater is also shown in the image on the right in Figure 8.21.

Junctions of LAST - LASTT couples have resulted in short circuit current values exceeding 8 A for a hot side temperature of $873 \mathrm{~K}\left(600^{\circ} \mathrm{C}\right)$ and cold side temperature of $305 \mathrm{~K}\left(32^{\circ} \mathrm{C}\right)$ (shown in Figure 8.22).
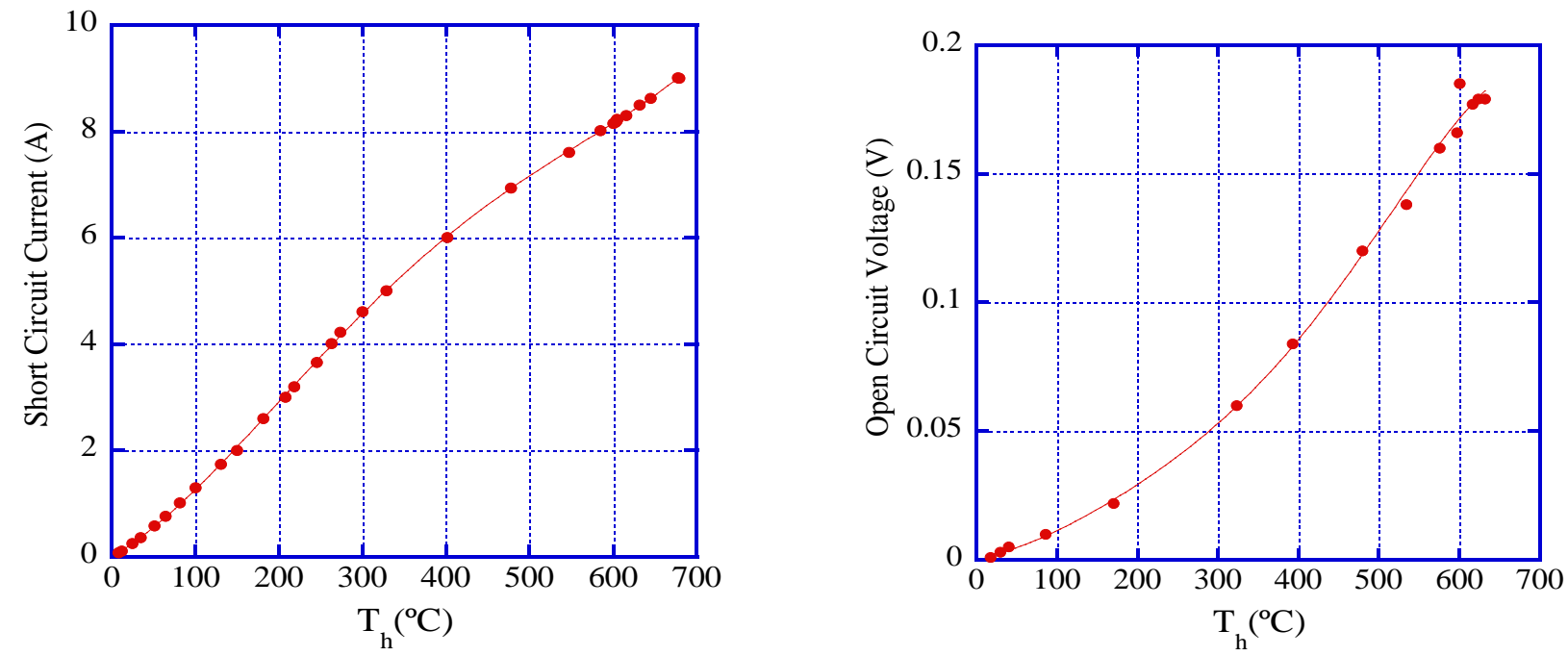

Figure 8.22. Short circuit current output for a couple module made from LAST and LASTT samples bonded to a stainless steel (316) electrode using a SN-Te bonding layer. 
Based on this bonding technique, the output power per couple has reached $450 \mathrm{~mW}$, which approached the $500 \mathrm{~mW}$ expected for zero contact resistance. Due to the relatively low thermal conductivity of these materials, the heat transfer requirements on the heat exchangers used with modules made from these materials is expected to be readily achievable with commercially available units.

Modules have been tested with a constant temperature gradient for over 72 hours and showed negligible change in the output short circuit current. Several temperature cycles have also been made on the same couple module with a small decrease in short circuit current (less than 10\%). We are continuing the work on these systems to investigate segmented structures with bismuth telluride samples for the cold side and cascaded module structures to construct high efficiency modules.

Modules fabricated from cast materials demonstrate their power output (Figure 8.23) limitations of the materials that come from the cracking. Contact studies for higher temperatures have been taken with the system described in Figure 8.24.
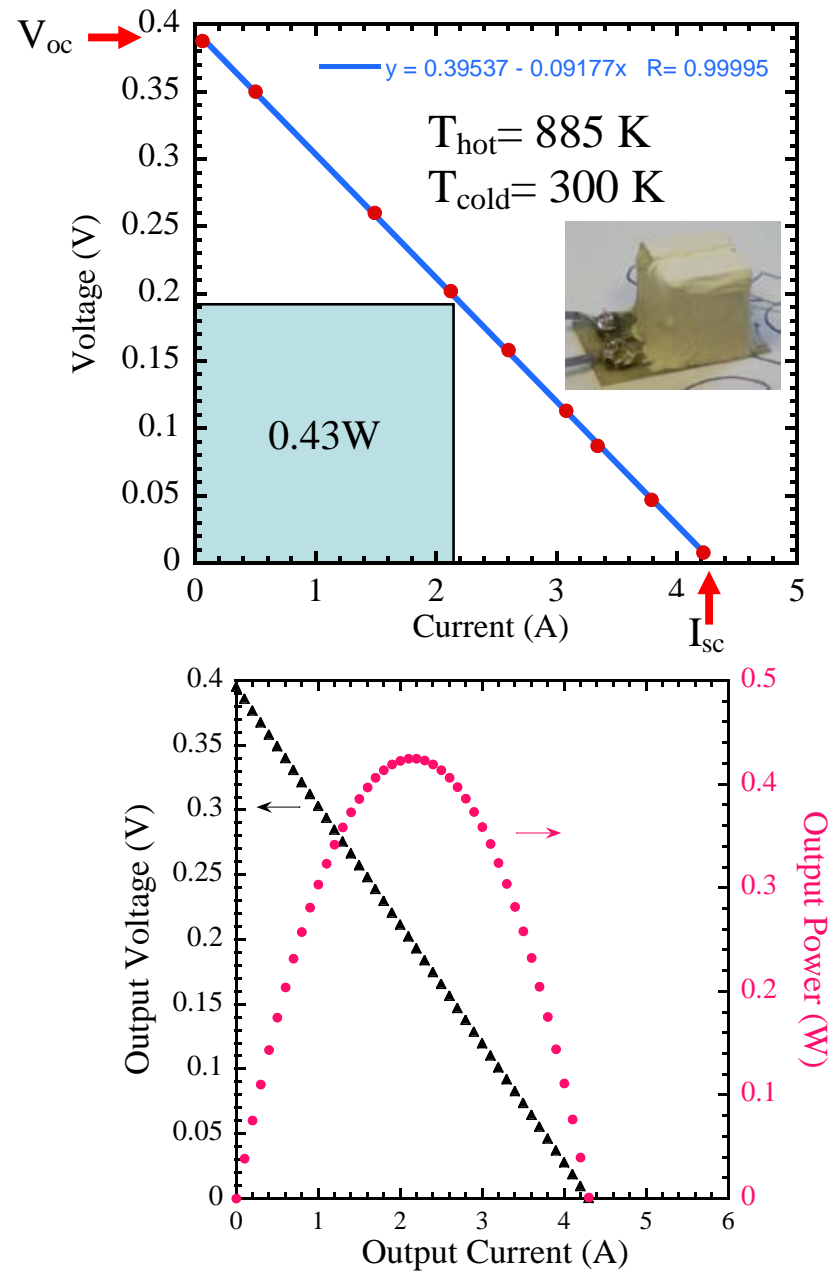
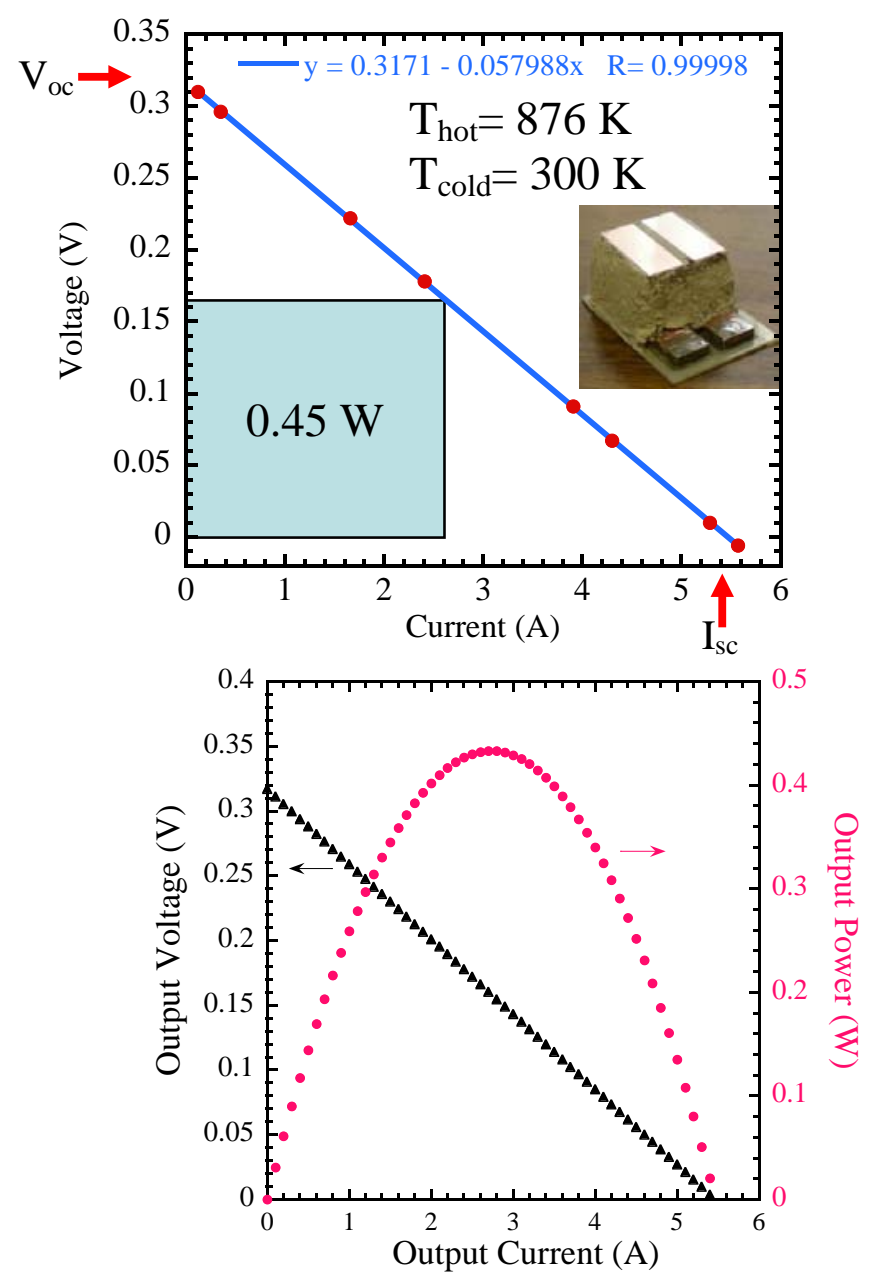

Figure 8.23. Four leg modules fabricated with cast materials of LAST and LASTT. 

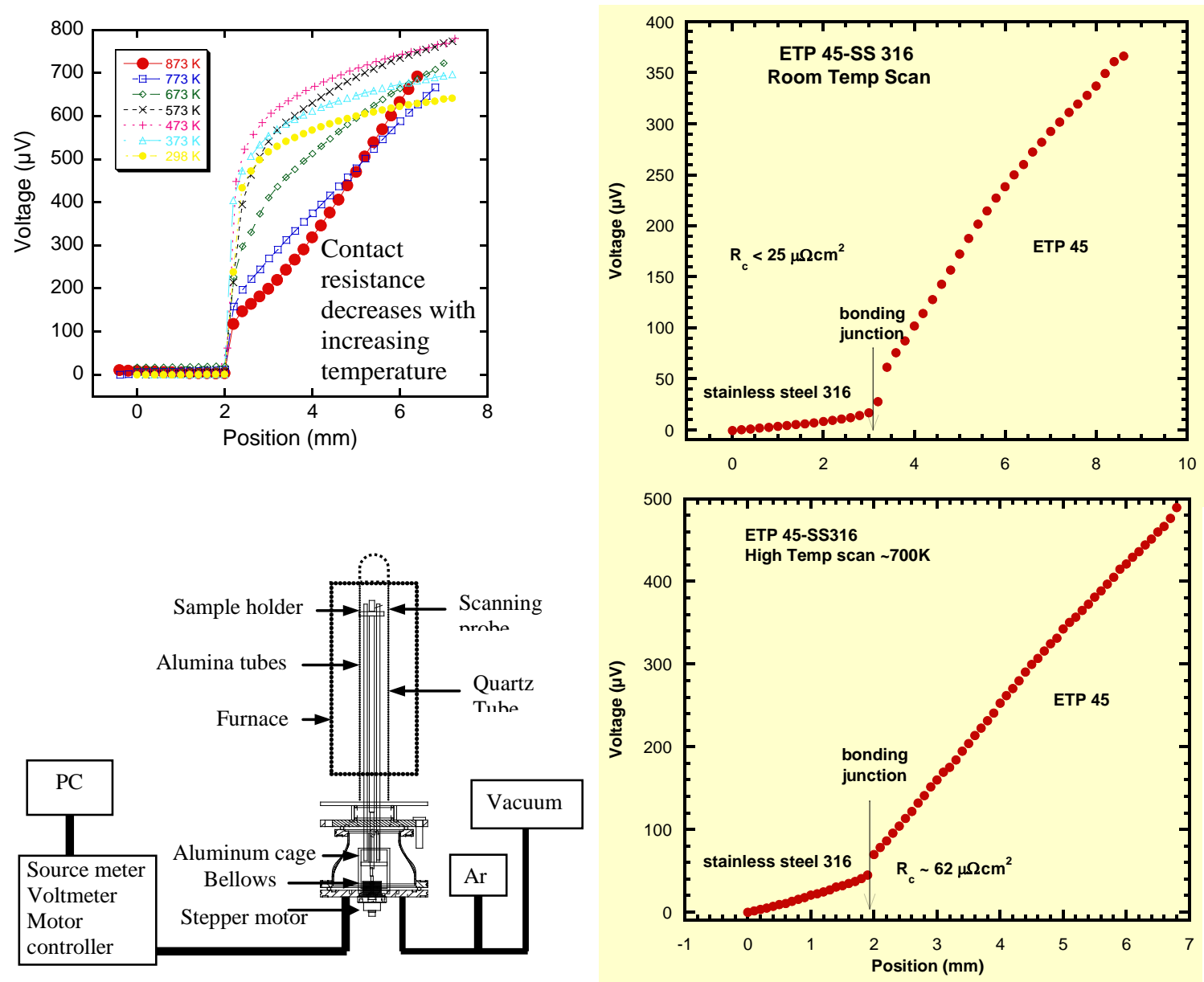

Figure 8.24. High temperature contact resistance measurement system and measurements. Several contacts have shown decreasing contact resistance with increasing temperature. 
A module measurement system for power output and efficiency measurements is shown in Figure 8.25.
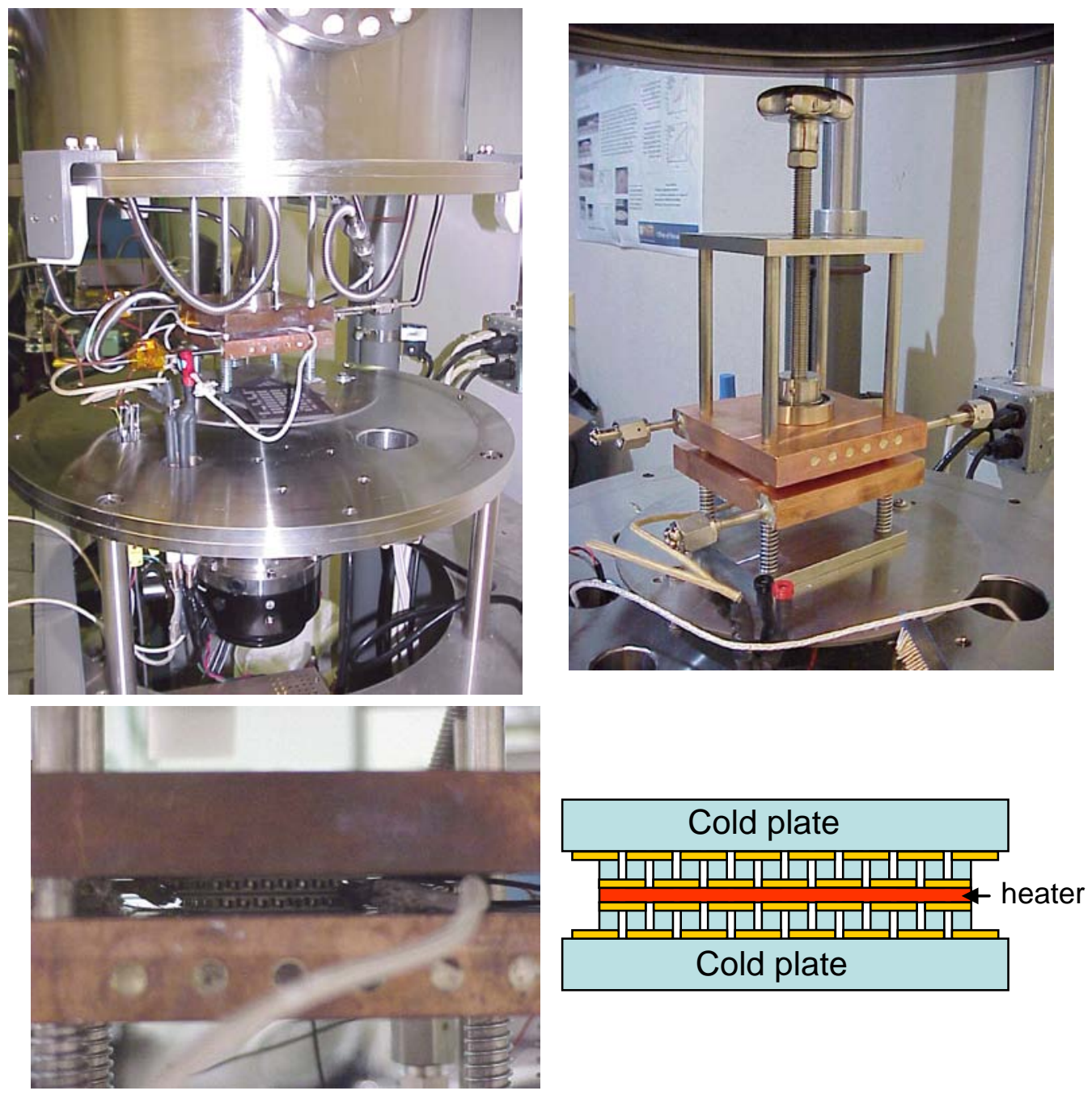

Figure 8.25. Module measurement system.

\section{Material Research - Investigation of PbTe - PbS Systems}

\subsection{Scale Up Studies and Reproducibility of PbTe - PbS Thermoelectric Materials}

The scale up studies described in this section were supported $80 \%$ by the DOE and $20 \%$ by the ONR. The material property characterization was supported $20 \%$ by DOE and $80 \%$ by ONR. For the system $\mathrm{PbTe}-\mathrm{PbS}$, nanostructures are formed by a precipitation process dependent on its composition and temperature. These precipitation phenomena are clearly marked by a miscibility gap (nucleation and growth) and chemical spinodal (spinodal decomposition) in the binary phase diagram. Toward the middle of the composition range $\left(0.30<\mathrm{X}<0.85\right.$ at $\left.500^{\circ} \mathrm{C}\right)$, phase segregation occurs through spinodal decomposition, characterized by unstable compositional fluctuations that extend over large areas. Toward the outer extremes of the composition range $\left(x>0.85\right.$ and $x<0.30$ at $500^{\circ} \mathrm{C}$ ), phase segregation occurs through nucleation and growth, characterized by large compositional differences that are small in scale. With annealing, these 
precipitates will coarsen into larger structures whose composition is determined by the wellunderstood Lever rule. Our work shows that solid solutions of $\mathrm{PbS}_{\mathrm{x}} \mathrm{Te}_{1-\mathrm{x}}(\mathrm{x}=0.08$ and 0.30$)$ can be synthesized and precipitation of the minor PbS phase is initiated upon heating. This provides a unique opportunity to compare the thermoelectric properties of the $\mathrm{PbTe}-\mathrm{PbS}$ system, both as a solid solution and a nanostructured composite.

For the material $\mathrm{PbTe}-\mathrm{PbS} 8 \%$, precipitation of $\mathrm{PbS}$ by nucleation and growth results in $\sim 50 \%$ decrease in lattice thermal conductivity from approximately $1 \mathrm{~W} / \mathrm{mK}$ to $0.4 \mathrm{~W} / \mathrm{mK}$ going from a solid solution to a nanostructured composite, respectively. In addition, the metastable nature of the material is clearly observed during precipitation. Conversely, $\mathrm{PbTe}-\mathrm{PbS} 30 \%$ increases slightly from essentially the amorphous limit. We show that in addition to a significant decrease in lattice thermal conductivity, the ZT of the material is improved by increases in both electron mobility and carrier concentration. This has yet to be observed for any thermoelectric material and shows that nanostructures not only have an effect of decreasing thermal conductivity, but also dramatically change the electronic structure of the material and result in a reproducibly high-ZT material.

\subsection{Structural Characterization (20\% DOE, $80 \%$ ONR)}

The PbTe - PbS system exhibits a miscibility gap that is defined by the plot of chemical potential (Gibbs free energy, $\mathrm{G}$ ) for a given isotherm across the composition range $\mathrm{x}$. If a sample of $\mathrm{PbTe}-\mathrm{PbS}$ is rapidly cooled from the melt to a lower temperature, the initial composition is expected to be completely homogeneous, i.e. a solid solution. Where the free energy plot has negative curvature $\left(\partial^{2} \mathrm{G} / \partial \mathrm{x}<0\right)$, the solid solution alloy is immediately unstable and free energy is decreased by periodic compositional fluctuations, characterized as spinodal decomposition. Spinodal decomposition is a unique thermodynamic process, as there is no activation energy barrier to the formation of the fluctuations. As such, it can be expected that a solid solution alloy of $\mathrm{PbTe}_{1-\mathrm{x}} \mathrm{S}_{\mathrm{x}}$ within the spinodal region will spontaneously decompose to yield a two phase PbTe - PbS material regardless of thermal treatment, although the speed of precipitation can be affected by differences in temperature.

Where the free energy plot has positive curvature $\left.\partial^{2} \mathrm{G} / \partial \mathrm{X}>0\right)$, the solid solution alloy is metastable and the free energy can only be decreased by precipitation of distinct nuclei of a second phase, characterized as nucleation and growth. In contrast to spinodal decomposition, the nucleation and growth phase transformation is limited by an activation energy barrier. For this reason, it can be expected that a solid solution alloy of $\mathrm{PbTe}_{1-\mathrm{x}} \mathrm{S}_{\mathrm{x}}$ within the nucleation and growth region will only exhibit precipitation once the requisite activation energy barrier is overcome, likely by thermal treatment.

\subsection{Powder X-Ray Diffraction (PXRD) (20\% DOE, 80\% ONR)}

To assure the synthesis of a solid solution of $\mathrm{PbTe}$ and $\mathrm{PbS}$, rapidly cooled samples were analyzed before and after heating. In each case, there is no PbS second phase evident by PXRD following the cooling. Heating the samples to $400^{\circ} \mathrm{C}$ even once causes significant precipitation of $\mathrm{PbS}$ (Figure 9.1). For each sample, the precipitation of $\mathrm{PbS}$ is evidenced by the movement of the major peaks toward smaller $\mathscr{Z}$ as well as the appearance of the PbS (200), (220) and (111) reflections. The corresponding shift to higher $2 \Theta$ with increasing $\mathrm{PbS}$ concentration suggests increased solid solubility of $\mathrm{PbS}$ in PbTe, showing that there still exists some solid-solution 
alloying between the two phases (Figure 9.2). Analysis of the bottom and top of the ingots showed complete homogeneity of PbTe and PbS phases without any composition gradient.

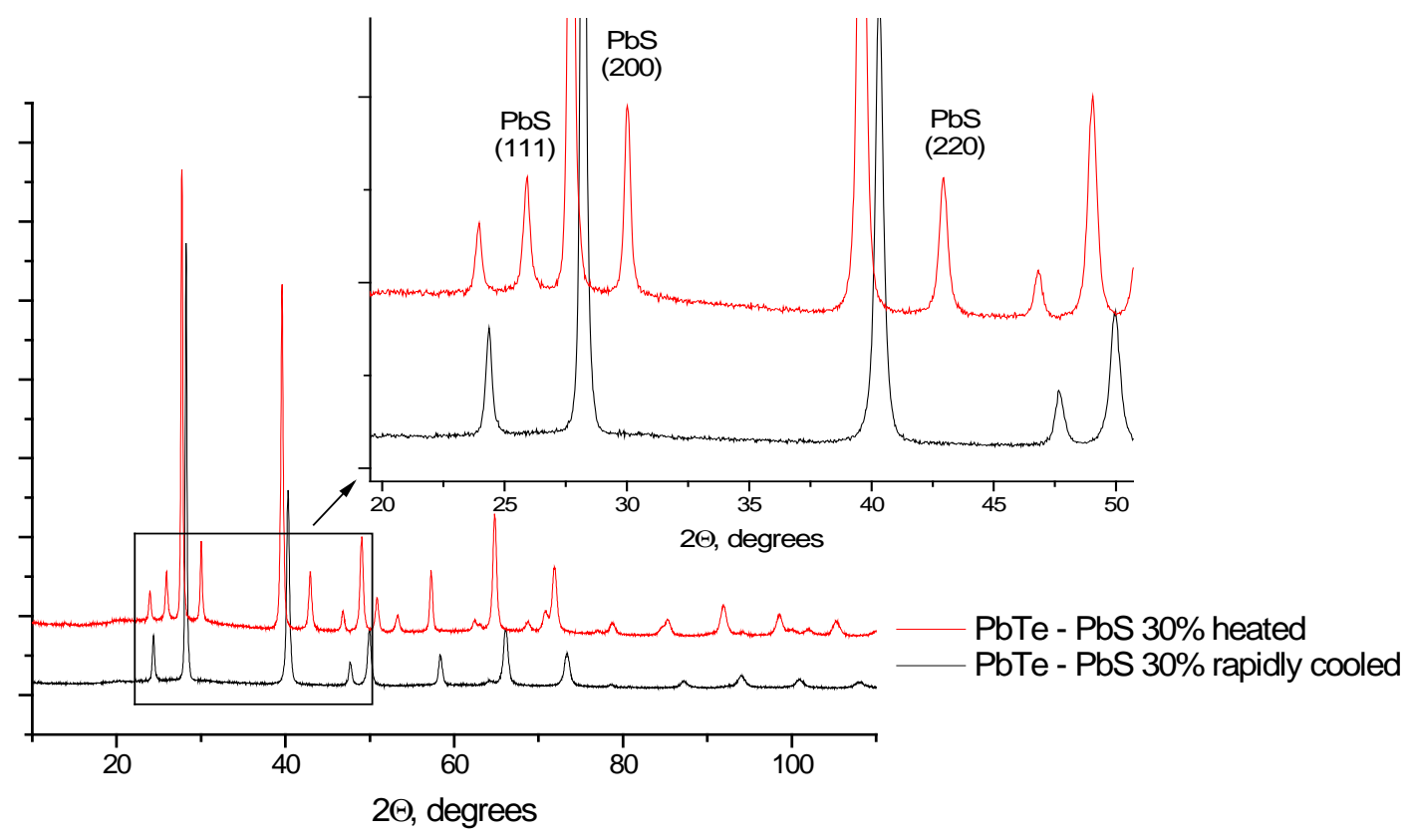

Figure 9.1. $\mathrm{PXRD}$ patterns of $\mathrm{PbTe}-\mathrm{PbS} 30 \%$ following rapid cool and after heating to $400^{\circ} \mathrm{C}$. The rapidly cooled material appears as a solid solution alloy, exhibiting a single cubic

$\mathrm{PbTe}$ phase. Following heating, a significant $\mathrm{PbS}$ second phase has precipitated from the PbTe matrix with a corresponding shift in the major peaks to lower $2 \Theta$, indicating a net increase in lattice parameter. The major PbS reflections are labeled in the inset. 


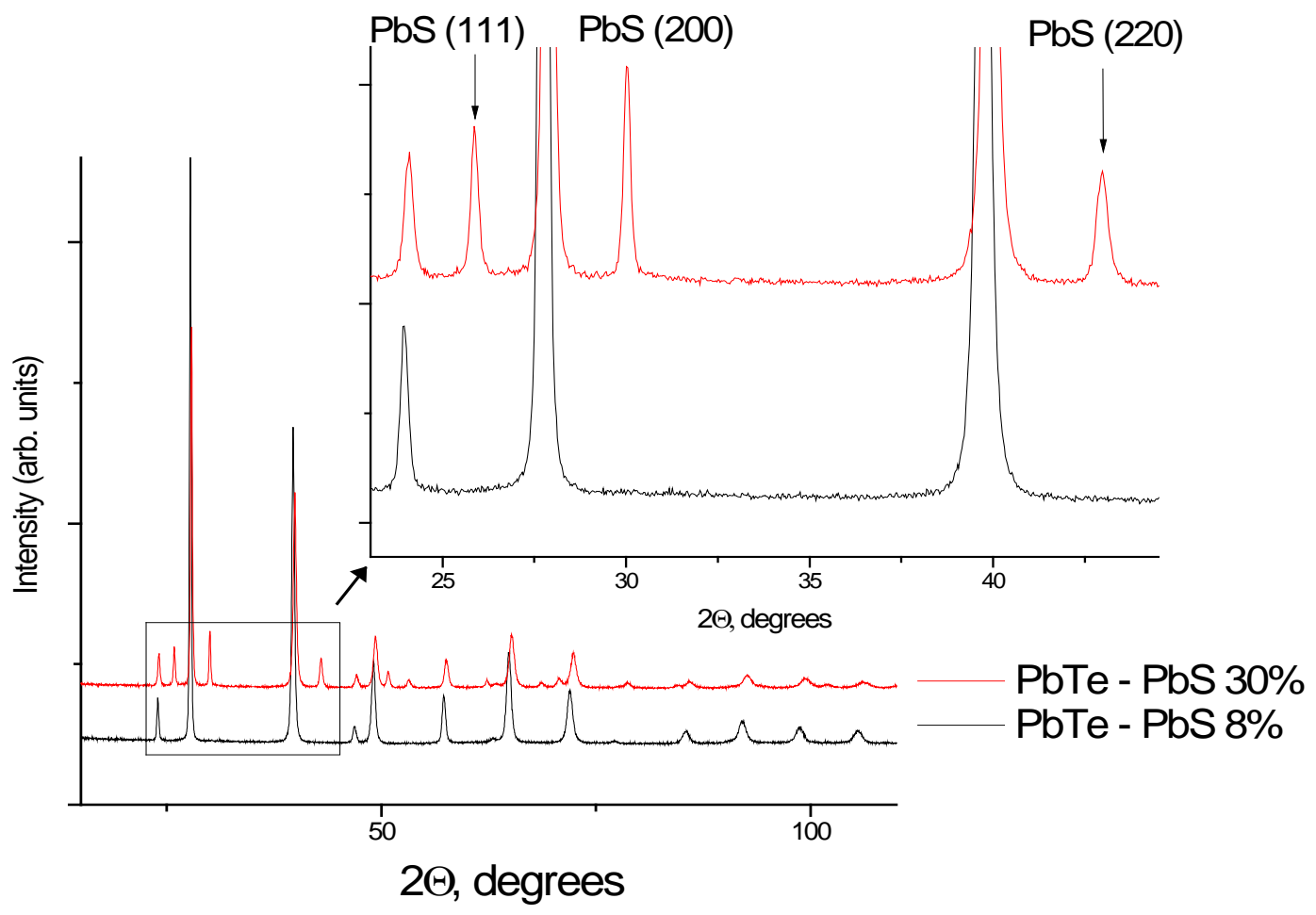

Figure 9.2. PXRD patterns of phase separated PbTe - PbS 8 and 30\%. By PXRD, the $\mathrm{PbS}$ phase is easier to detect in concentrations larger than $8 \%$.

\subsection{SEM/HRTEM - (20\% DOE, 80\% ONR)}

Rapidly cooled samples were verified by SEM and HRTEM and were composed almost exclusively of a single phase alloy of $\mathrm{PbS}_{1-\mathrm{x}} \mathrm{Te}_{\mathrm{x}}$, with very few precipitates of $\mathrm{PbS}$ observed (Figure 9.3a). Atomic-level resolution images displayed in Figure 9.3b show mostly solid solution behavior and the local structure appears to show complete homogeneity of $\mathrm{S}$ and Te within the matrix. Selected area electron diffraction (SAED) of all samples reveals a single phase material, Figure 9.3c.
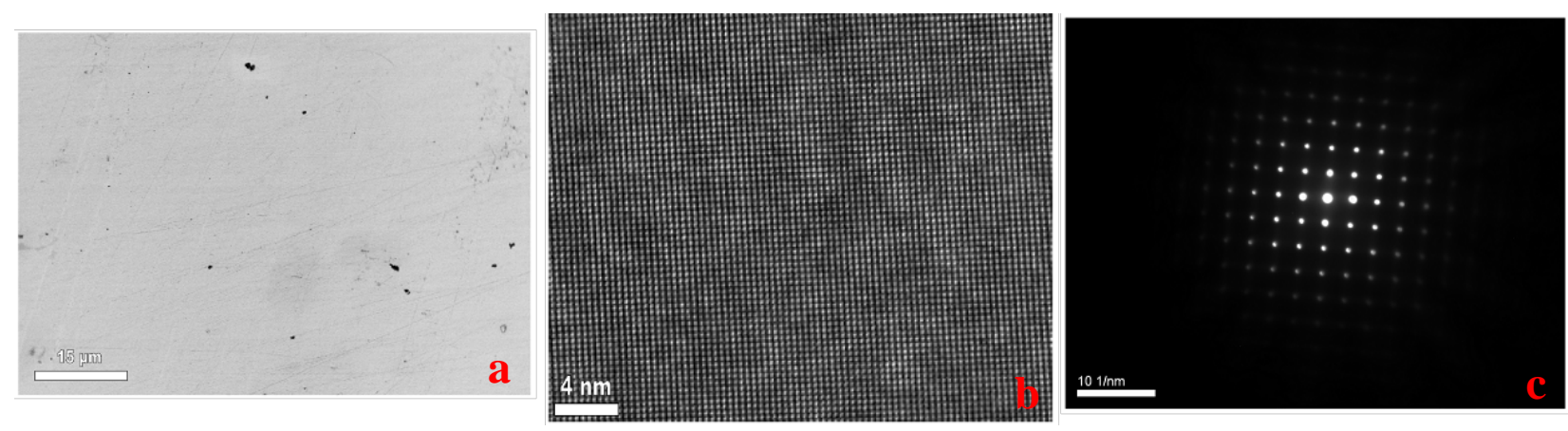

Figure 9.3 - Microstructure of rapidly cooled $\mathrm{PbTe}$ - PbS 30\%, exhibiting near solid-solution behavior. For all PbTe - PbS compositions investigated, observation of the solid solution of the materials looks similar. The material appears almost completely single phase in a) SEM micrograph, b) HRTEM image, c) SAED showing single-phase material.

b) Dark spots in a) are hole defects in the sample surface. 
As expected, annealed samples show vastly different microstructure. For $\mathrm{PbTe}-\mathrm{PbS} 8 \%$, Figure 9.4a shows the average size of the particles observed are approximately $100 \mathrm{~nm}$ in diameter, although there exists a range of particle sizes ranging from approximately $2 \mathrm{~nm}$ to 10 $\mu \mathrm{m}$. EDS analysis of the particles reveal them to be composed mostly of PbS. HRTEM images reveal compositional inhomogeneity within the particles in the form of smaller particles on the order of 2-10 nm (Figure 9.4b). The exact composition of the smaller particles is not yet known, but they are likely either discrete particles of $\mathrm{PbTe}$ embedded within the $\mathrm{PbS}$ or some intermediate alloy between $\mathrm{PbTe}$ and $\mathrm{PbS}$. A HRTEM image of a PbS nanoparticle in $\mathrm{PbTe}-$ $\mathrm{PbS}$ 8\% is shown in Figure 9.4c. Selected area electron diffraction (SAED) of the particles reveals an identifiable $\mathrm{PbS}$ second phase that is fully coherent within the PbTe matrix, Figure 7.4d.
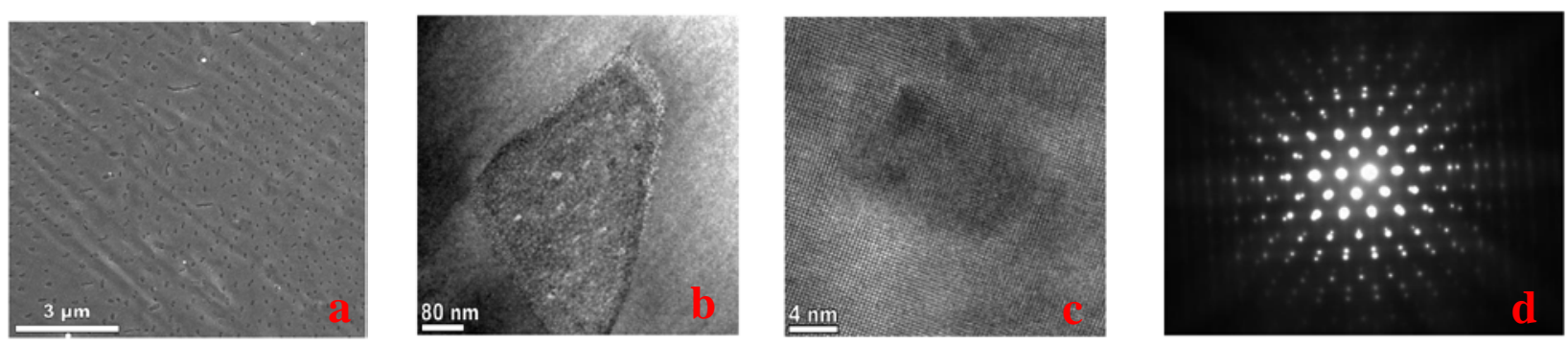

Figure 9.4. Typical microstructure of $\mathrm{PbTe}-\mathrm{PbS} 8 \%$ samples phase separated by nucleation and growth. a) SEM image of PbTe - PbS 8\% showing particles of PbS (dark spots) embedded throughout the PbTe matrix of average diameter $\sim 100 \mathrm{~nm}$, b) low magnification HRTEM image of PbS particle showing nanoscale inhomogeneities within the PbS particle, c) HRTEM image of a PbS nanoparticle in PbTe - PbS 8\%, d) SAED of image shown in b) showing reflections for $\mathrm{PbTe}$ and $\mathrm{PbS}$. The two phases are completely coherent.

The microstructure of $\mathrm{PbTe}$ - PbS 30\% shows that spinodal decomposition will occur throughout the sample, resulting in bands of PbS approximately 20-100 nm across that will extend for microns in length (Figure 9.5a). Previous work of this system reported compositional fluctuations on the order of $2 \mathrm{~nm}$ to persist throughout the PbS bands. It has been determined that this is an effect of Moiré fringes that become less apparent towards the edge of the electrontransparent foil (Figure 9.5b). Still, there exists some level of local inhomogeneity within the spinodally decomposed bands of PbS, as particles of a similar size and appearance as those observed in $\mathrm{PbTe}-\mathrm{PbS}$ 8\% were observed for $\mathrm{PbTe}-\mathrm{PbS} 30 \%$ (Figure 9.5c). The $\mathrm{PbS}$ bands are fully coherent within the PbTe matrix (Figure 9.5d) as shown by SAED. 

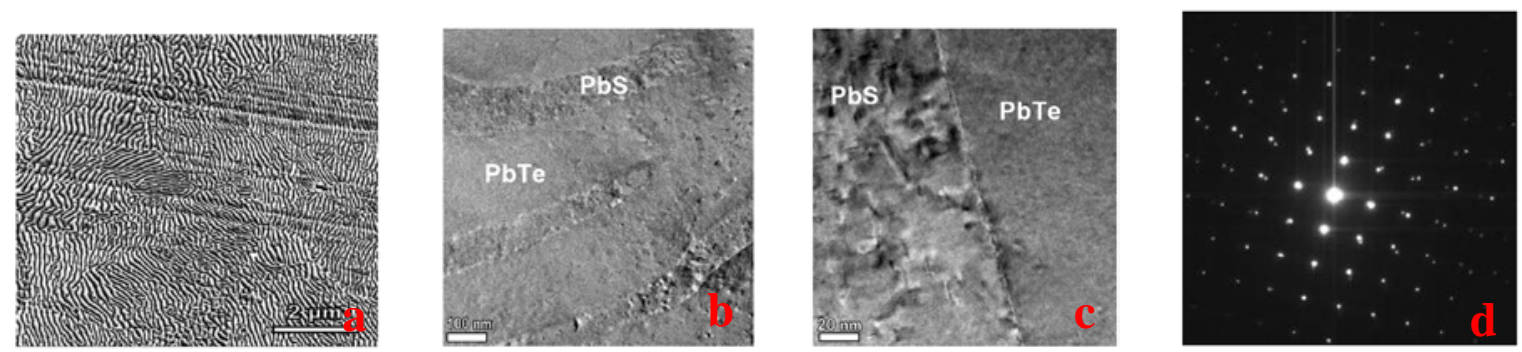

Figure 9.5. Typical microstructure of $\mathrm{PbTe}-\mathrm{PbS} 30 \%$ phase separated by spinodal decomposition. a) SEM image of $\mathrm{PbTe}-\mathrm{PbS} 30 \%$ showing bands of $\mathrm{PbS}$ (dark regions) throughout the PbTe matrix, b) low magnification HRTEM image of PbS-rich bands in $\mathrm{PbTe}-\mathrm{PbS} 30 \%$ showing small nanoparticles within both $\mathrm{PbTe}$ and $\mathrm{PbS}$ regions,

c) HRTEM image of the PbTe/PbS interface shows compositional inhomogeneities present within the spinodally decomposed PbS band, d) SAED of image from b) shows reflections for $\mathrm{PbTe}$ and $\mathrm{PbS}$ to be completely coherent.

\subsection{Thermoelectric Properties}

Electrical conductivity decreases monotonically with increasing $\mathrm{PbS}$ concentration in $\mathrm{PbTe}-$ $\mathrm{PbS}$, with room temperature values around $2000 \mathrm{~S} / \mathrm{cm}$ for $8 \%, 1500 \mathrm{~S} / \mathrm{cm}$ for $16 \%$, and 1200 $\mathrm{S} / \mathrm{cm}$ for $30 \%$ using $0.055 \mathrm{~mol}^{2} \mathrm{PbI}_{2}$ as dopant. However, it was found that precipitation effects are less apparent as the overall carrier concentration of the system is increased, so for certain samples less dopant was used to facilitate in observing precipitation phenomena. The precipitation of $\mathrm{PbS}$ can be described best through the deviation from the expected $\rho=1 / \sigma \alpha \mathrm{T}^{\lambda}$ power law for PbTe- based materials. Figure 9.6a shows that for PbTe-PbS 8\%, the electrical conductivity follows the power law with $\lambda=2.17$ until $400 \mathrm{~K}$, when precipitation of $\mathrm{PbS}$ nanostructures alters the electronic structure of the material and results in a complete deviation from the power law trend. Following the precipitation of $\mathrm{PbS}$, the material stabilizes and the electrical conductivity returns to the expected trend, with $\lambda=2.28$. For $\mathrm{PbTe}-\mathrm{PbS} 8 \%$, precipitation of $\mathrm{PbS}$ results in increased electrical conductivity and decreased thermopower, shown in Figure 9.6a and Figure 9.6b. Following PbS precipitation, the electrical transport appears unchanged with repeated high-temperature cycling, indicating that the precipitation of $\mathrm{PbS}$ is complete and the sample has reached thermodynamic equilibrium.

Conversely, $\mathrm{PbTe}$ - $\mathrm{PbS} 30 \%$ decreases in electrical conductivity and increases in thermopower, (Figure 9.6c) but without the clear transition appearing between 400 and $500 \mathrm{~K}$. Rather, the temperature dependence of the electrical conductivity appears to change over the course of the measurement, resulting in a net decrease in electrical conductivity following phase separation. This suggests that there is a constant change in electrical transport over the measurement, consistent with an unstable phase transformation such as spinodal decomposition where there exists no energy barrier to nucleation. Stability of the properties in subsequent heating and cooling measurements indicate that the material has reached thermodynamic equilibrium (Figure 9.6c). Figure 9.6d shows thermopower data for $\mathrm{PbTe}-\mathrm{PbS} 30 \%$ samples as a function of temperature. The unstable spinodal decomposition phase transformation occurs over the entire temperature range. Following the initial high-temperature cycling (i.e. following $\mathrm{PbS}$ precipitation), the electrical properties of both samples stabilize. 


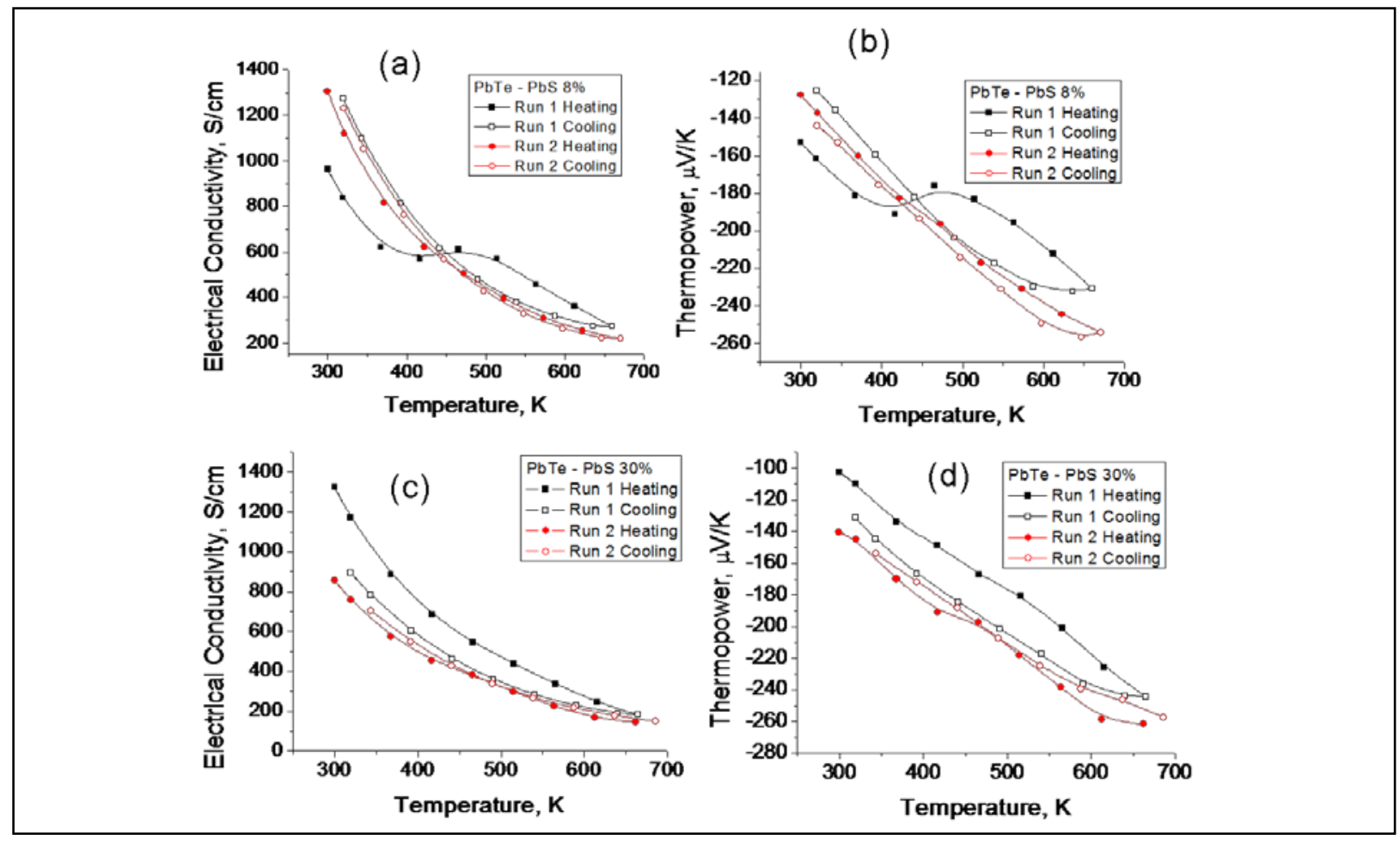

Figure 9.6. Temperature-dependent a) electrical conductivity and b) thermopower of $\mathrm{PbTe}-\mathrm{PbS} 8 \%$. The deviation in the expected power law dependence is the result of the metastable phase transformation $\sim 500 \mathrm{~K}$ consistent with nucleation and growth.

Temperature-dependent c) electrical conductivity and d) thermopower of PbTe - PbS 30\%.

The change in electrical conductivity can be attributed both to changes in Hall electron mobility and carrier concentration, from the relation $\sigma=n e \mu_{H}$ where $n$ is the carrier concentration, $e$ is the fundamental electron charge, and $\mu_{H}$ is Hall electron mobility. Hall effect measurements were used to determine the Hall coefficient $R_{H}$ and the electron concentration was calculated through the equation $n=1 / e R_{H}$. The results for electron mobility and carrier concentration for PbTe $\mathrm{PbS}$ 8\% are shown in Figure 9.7a and Figure 9.7.b. The increase in electron mobility at room temperature from $\sim 1000 \mathrm{~cm}^{2} / \mathrm{Vs}$ to $\sim 1250 \mathrm{~cm}^{2} /$ Vs following PbS precipitation suggests that the formation of larger regions of $\mathrm{PbTe}$ and $\mathrm{PbS}$ reduces the amount of electron scattering from ionized impurities, similar to previously reported phenomena. More surprising is the considerable increase in electron concentration, from $\sim 7 \times 10^{18} \mathrm{~cm}^{-3}$ for solid solution $\mathrm{PbTe}-\mathrm{PbS}$ $8 \%$ to $\sim 1.2 \times 10^{19} \mathrm{~cm}^{-3}$ for composite $\mathrm{PbTe}-\mathrm{PbS} 8 \%$, an increase of over $40 \%$ (Figure 9.7b). This report marks the first time such an effect has been observed. Indeed, the precipitation of $\mathrm{PbS}$ particles in this system results in not only an increase in the electron mobility, but a substantial increase in the overall number of electrons available for conduction in the PbTe $\mathrm{PbS}$ 8\% composition, which exhibits precipitation from nucleation and growth. This result suggests that there exists a significant change in the electronic structure of the material as it is phase separated, in contrast to previous work which largely attributed nanoscale composite thermoelectrics to only alter thermal transport. The implications of this finding are discussed later. Conversely, the corresponding decrease in the carrier concentration for the PbTe - PbS $30 \%$ sample suggests that the spinodal decomposition process results in a decreased number of electrons available for conduction. 

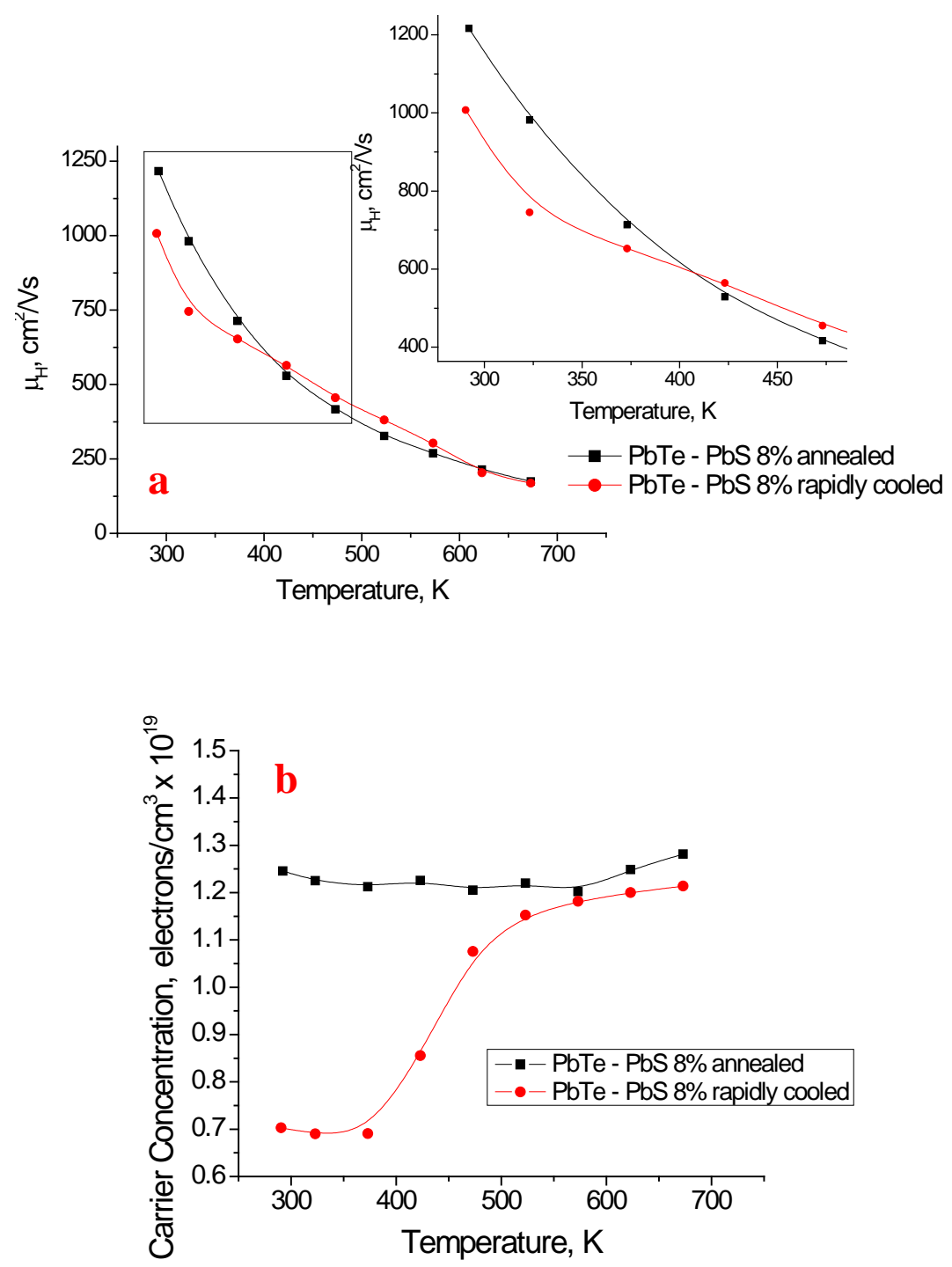

Figure 9.7. a) Hall mobility of $\mathrm{PbTe}-\mathrm{PbS} 8 \%$ rapidly cooled and annealed. The precipitation of PbS results in an increase in electron mobility from $1000 \mathrm{~cm}^{2} / \mathrm{Vs}$ to $1250 \mathrm{~cm} / \mathrm{Vs}$. b) Carrier concentration for $\mathrm{PbTe}-\mathrm{PbS} 8 \%$ rapidly cooled and annealed. The carrier concentration of the material increases considerably following precipitation of $\mathrm{PbS}$.

\subsection{Thermal Transport}

Thermal conductivity measurements of disc-shaped samples taken from the same ingots as those reported previously exhibit the same precipitation effects observed for electrical transport. While the heating profiles of the electrical and thermal transport measurements are not exactly the same, the speed and scale of the heating profiles were kept as close as possible. The characteristic fluctuation in the $1 / \sigma \alpha \mathrm{T}^{\lambda}$ scaling law is again observed for the $\mathrm{PbTe}-\mathrm{PbS} 8 \%$ samples indicating nucleation and growth phenomena, while the $\mathrm{PbTe}-\mathrm{PbS} 30 \%$ exhibit continual change over the temperature range, indicating spinodal decomposition, Figure 9.8a 
and Figure 9.8b. In addition, this shows that the precipitation of PbS is observed irrespective of measurement system, and that it is phenomenon inherent to this class of materials.

The lattice thermal conductivity was calculated using the WiedemanN-Franz law $\kappa_{\text {elec }}=\sigma \cdot \mathrm{T} \bullet$ $\mathrm{L}_{0}$, where $\mathrm{L}_{\mathrm{o}}=2.45 \cdot 10^{-5} \mathrm{Wcm}^{2} / \mathrm{K}^{2} \Omega$. For $\mathrm{PbTe}-\mathrm{PbS} 8 \%$, the lattice thermal conductivity falls sharply from $400-500 \mathrm{~K}$ by over $50 \%$, from approximately $1 \mathrm{~W} / \mathrm{mK}$ to $0.4 \mathrm{~W} / \mathrm{mK}$, as a result of nano- and microscale PbS precipitation (Figure 9.8c). Following the phase transformation, the lattice thermal conductivity remains unchanged and the sample can be cycled repeatedly without a change in properties as with the electrical transport.
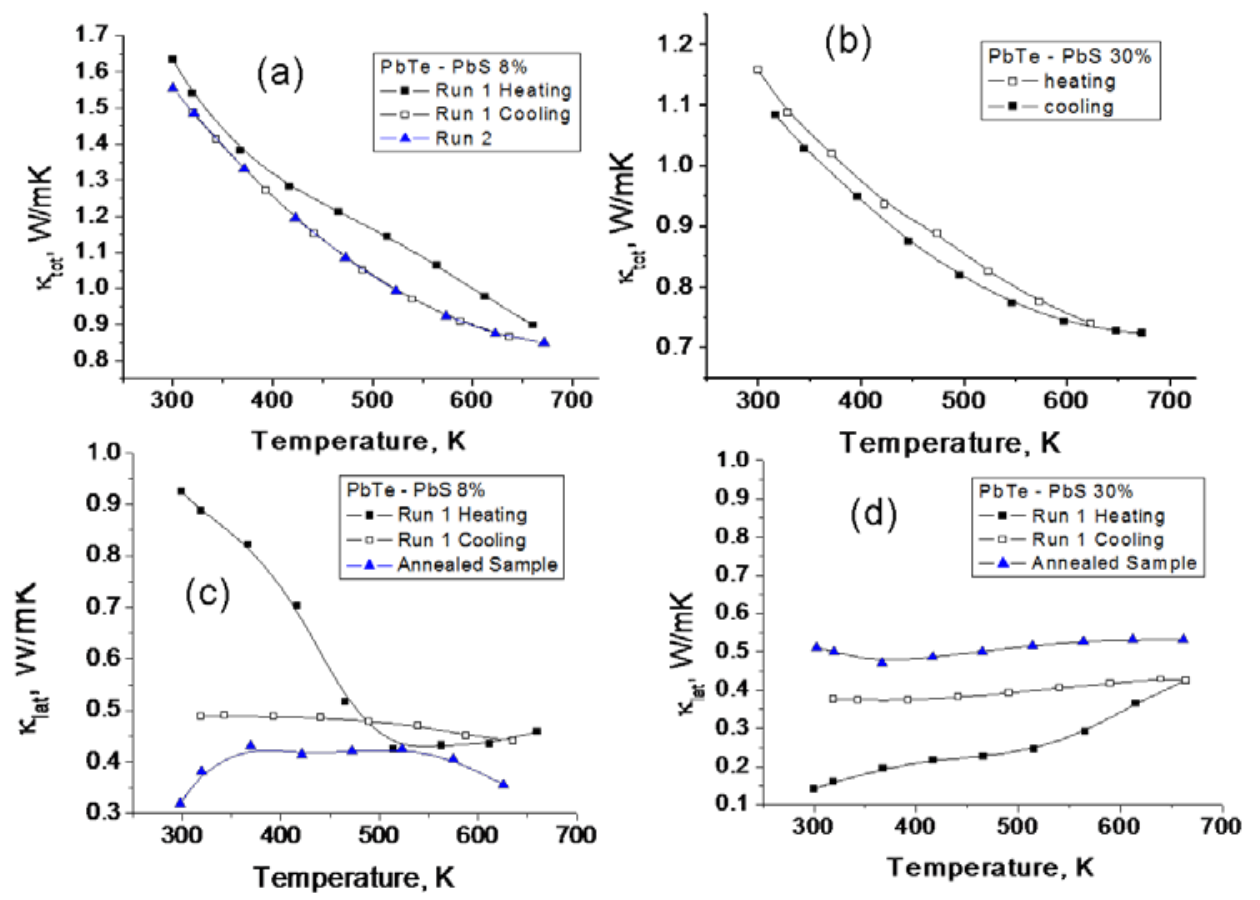

Figure 9.8. a) Total thermal conductivity of $\mathrm{PbTe}-\mathrm{PbS} 8 \%$, b) total thermal conductivity of $\mathrm{PbTe}-\mathrm{PbS} 30 \%$, c) lattice thermal conductivity of PbTe - PbS 8\%, d) lattice thermal conductivity of PbTe - PbS 30\%. The same precipitation events observed in electrical transport are observed again in thermal transport. The precipitation of $\mathrm{PbS}$ in $\mathrm{PbTe}-\mathrm{PbS} 8 \%$ results in a significant reduction of lattice thermal conductivity by almost 50\%.

For PbTe - PbS 30\%, the lattice thermal conductivity follows a different trend. Significantly lower lattice thermal conductivity is calculated for the solid solution at room temperature, likely indicating that a combination of the solid-solution alloying and the early spinodal decomposition are contributing in tandem. Following precipitation of $\mathrm{PbS}$, however, the lattice thermal conductivity increases to around $0.5 \mathrm{~W} / \mathrm{mK}$ (Figure 9.8d). Again, once precipitation of the $\mathrm{PbS}$ phase is completed, the properties do not change with continual thermal cycling from room temperature to $750 \mathrm{~K}$.

It should be noted that for both samples, the significant reduction in lattice thermal conductivity appears to be a result of changes to both the thermal and electrical structure of the materials. The 
calculation of the lattice thermal conductivity is intimately related to the electrical conductivity, which we show to change following precipitation of PbS. It is likely that the Lorenz used is not accurate, however as previously reported we would expect that heavily doped degenerate semiconductors to exhibit a $\mathrm{L}_{0}$ close to $2.45 \cdot 10^{-5} \mathrm{~W} / \mathrm{cm}^{2} \mathrm{~K} \Omega$ at room temperature.

Calculated values of ZT are shown in Figure 9.9a. The ZT for both materials is increased over that of just PbTe, with a ZT of approximately 1 for PbTe - PbS 30\% and 1.3 for PbTe - PbS $8 \%$. The $\mathrm{PbTe}-\mathrm{PbS} 8 \%$ material is very reproducible, consistently resulting in high ZT (Figure 9.9b). To that effect, large-scale ( $100 \mathrm{~g})$ batches of the PbTe - PbS $8 \%$ have been successfully synthesized that also exhibit enhanced ZT (Figure 9.9b).

The ability to increase the scale and excellent reproducibility of this material system enhances its potential for use in practical thermoelectric applications. We achieved the reproducibility of this system and ability to scale-up the material to batches $\sim 100 \mathrm{~g}$.

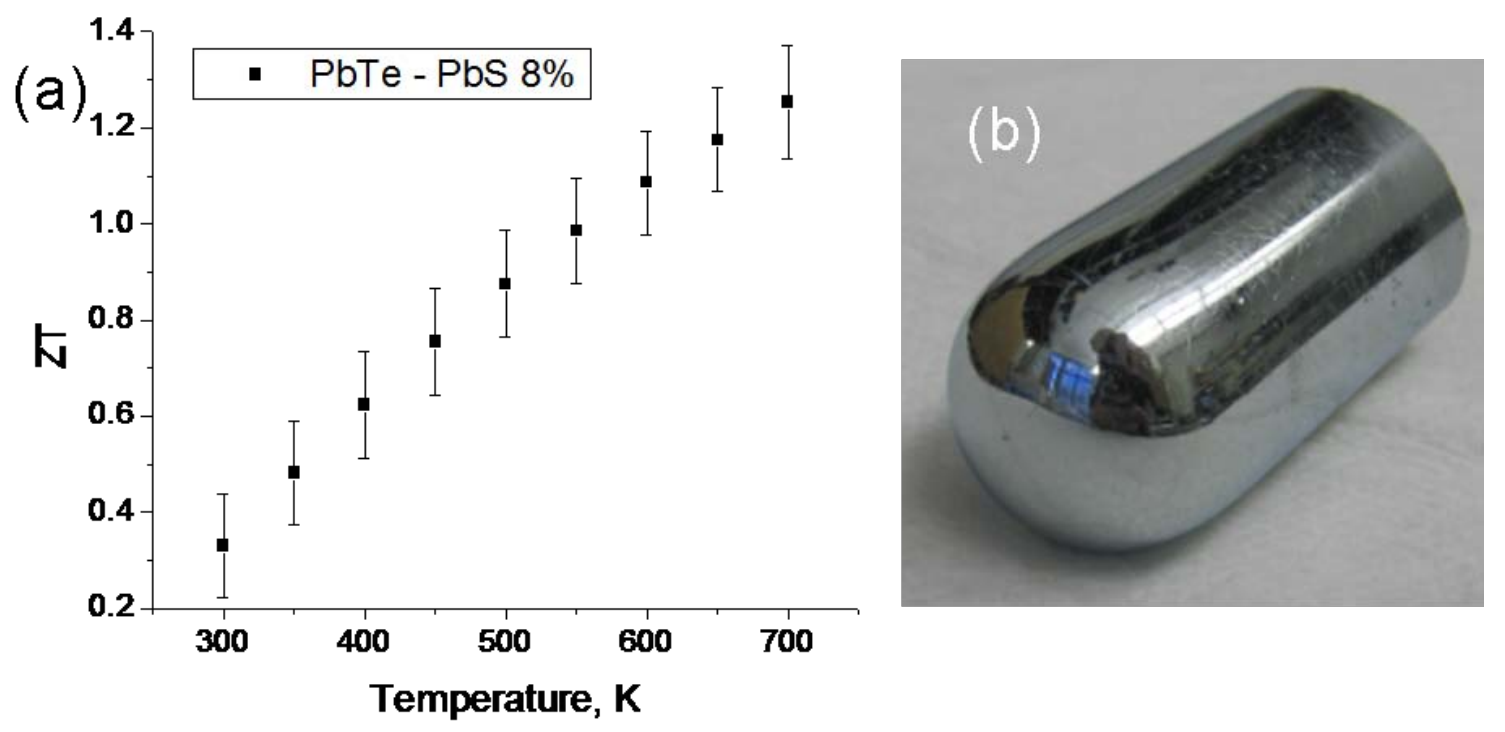

Figure 9.9. a) ZT range of PbTe - PbS 8\%, data acquired from 11 individually prepared ingots measured independently, with an average of 1.2. Error bars show \pm one standard deviation, b) picture of large-scale ( 100 g) ingot of PbTe - PbS 8\%. Large-scale ingots show good agreement with reported results (1 inch diameter).

\section{Powder Processing and Mechanical Properties of Skutterudite}

The work done by Professor Case's group involved the mechanical characterization of thermoelectric materials. Initially, the mechanical characterization work focused on LAST (Lead-antimony-silver-tellurium) and LASTT (Lead-antimony-silver-tellurium-tin) thermoelectric materials since it was those materials that were the first candidates for the thermoelectric materials to be used in the thermoelectric generators in this study. However, after this project's work on mechanical properties (as well as thermoelectric properties) determined that LAST and LASTT were not yet sufficiently developed to allow their immediate inclusion in 
sufficient thermoelectric generators, the research turned to skutterudite materials. Thus, while this report includes a brief statement of the work done on LAST and LASTT, the majority of this report is focused research on the mechanical characterization of the skutterudite materials. Journal papers and talks generated by LAST and LASTT research, along with the skutterudite research are listed in Appendix A.

\subsection{Research on LAST (Lead-antimony-silver-tellurium) and LASTT (Lead-antimony- silver-tellurium-tin) Thermoelectric Materials}

The hardness of LAST and LASTT was investigated as a function of porosity and grain size, along with the composition and temperature dependence of the elastic moduli. In addition, the fracture strength and microstructure of LAST and LASTT was investigated. During the entire study of these lead-telluride based materials, powder processing and hot pressing of LAST and LASTT was studied with Professor Case's group mainly responsible for the powder processing along with Edward Timm, a Specialist working with Professor Harold Schock. The research done on LAST and LASTT was performed by graduate students Fei Ren, Jennifer Ni and Bradley Hall. The journal papers and talks generated during the LAST and LASTT studies along with papers and talks dealing with the skutterudite research are listed in Appendix A.

\subsection{Overview for the Skutterudite Mechanical Characterization}

Thermoelectric materials are semiconductor materials that can generate an electric current from a thermal gradient. Assembled into a generator, thermoelectric materials may use waste heat to recover energy as electricity. To be used in a device, the thermoelectric material must be able to withstand the applied thermal and mechanical forces without failure. The powder processing, microstructure, elastic moduli, thermal expansion and hardness properties were measured for three thermoelectric skutterudite materials: two N-type skutterudite materials of composition $\mathrm{Co}_{0.95} \mathrm{Pd}_{0.05} \mathrm{Te}_{0.05} \mathrm{Sb}_{3}$, either with or without doping with 0.1 atomic \% cerium and a P-type skutterudite of composition $\mathrm{Ce}_{0.9} \mathrm{Fe}_{3.5} \mathrm{Co}_{0.5} \mathrm{Sb}_{12}$. The ball milling portion of powder processing was most efficiently accomplished in one step, with ethanol producing a powder that was hot pressed into billets with an average grain size of less than $2 \mu \mathrm{m}$. Elastic moduli were determined for all billets, with Young's modulus approximately 135 GPa for N-type, and 129 GPa for Ptype, varying according to porosity and composition. Moduli decreased linearly with temperature until $523 \mathrm{~K}$ to $623 \mathrm{~K}$, above which a viscoelastic region began and moduli decreased more rapidly. The bulk coefficient of thermal expansion (CTE) was $10.0 \times 10^{-6} \mathrm{~K}^{-1}$ for noncerium doped N-type, $11.2 \times 10^{-6} \mathrm{~K}^{-1}$ for cerium doped N-type, and $13.0 \times 10^{-6} \mathrm{~K}^{-1}$ for P-type. The P-type material had a lattice parameter of $9.1241 \AA$ at $303 \mathrm{~K}$. The CTE value determined by $\mathrm{x}$-ray diffraction was the same as the CTE measured from the bulk specimen. A new phase appeared in X-ray diffraction of the P-type material at $603 \mathrm{~K}$ and remains stable after cooling.

\subsection{Introduction}

The work presented in this report on the N-type skutterudite, $\mathrm{Co}_{0.95} \mathrm{Pd}_{0.05} \mathrm{Te}_{0.05} \mathrm{Sb}_{3}$, both with and without 0.1 atomic \% cerium dopant, and the P-type skutterudite, $\mathrm{Ce}_{0.9} \mathrm{Fe}_{3.5} \mathrm{Co}_{0.5} \mathrm{Sb}_{12}$, may be broadly categorized in three areas, (i) processing and microstructure, (ii) room temperature mechanical properties, and (iii) mechanical properties as a function of temperature. 


\subsection{Experimental Procedure}

Powder processing was accomplished in an argon atmosphere glove box with a mechanical mortar and pestle (Retsch RM200, Retsch GmbH, Haan, Germany) and ball mill (Retsch PM100). Microstructure was evaluated by SEM microscopy (JEOL 6400, JEOL Ltd., and Japan). Elastic moduli were determined by resonant ultrasound spectroscopy (Quasar RUSpec, Quasar International, and Albuquerque, NM) and nanoindentation (Nanoindenter XP with CSM/LFM, MTS, and Oak Ridge, TN). Hardness was measured using microindentation (Buehler Semimacro Indenter, Lake Bluff, IL, and Shimadzu HMV-2000, Kyoto, Japan) and nanoindentation (Nanoindenter XP with CSM/LFM, MTS, Oak Ridge, TN). Thermal expansion was measured using a thermomechanical analyzer (Q400, TA Instruments, New Castle, DE). Lattice parameter was measured by X-ray diffraction (PANalytical PW3040-PRO, Serial \#DY1331, PANalytical B. V., Almeno, Netherlands).

The powder processing began with crushing, grinding and regrinding the ingot until all powder passed through a $75 \mu \mathrm{m}$ sieve. The powder was then processed to reduce the particle size via ball milling (Appendix B).

Milling evolved from a two step process with significant scraping of the mill jar after each milling to a one step process. The single step process involved reduced powder caking and scraping of the mill jar and therefore less powder lost during processing. Ball milling was initially performed by a process developed for LAST (Lead-antimony-silver-tellurium) thermoelectric materials [11], with a 3 hour dry milling at 150 RPM followed by a 6 hour wet milling (in hexane) at 120 RPM. Later powder batches that employed wet milling with ethanol resulted in reduced caking and easier processing of the powder (Appendix B).

Beginning with batch 2 of the third ingot processed, JSpSKD-15, the 3 hour dry milling was eliminated and only wet milling was used. To achieve similar or better results without the dry milling step, the milling time was increased to 9 hours. Increasing milling time also increased evaporation of the ethanol, requiring an increase from $25 \mathrm{~mL}$ to $42 \mathrm{~mL}$ in the amount of ethanol initially loaded into the milling jar. In addition, eliminating dry milling reduced the manual handling of the powder between steps; ethanol use decreased the caking of powder and increased the powder recovered after each milling.

\subsection{Microstructural Analysis}

Microstructural evaluation of the milled powders was done by evaluating a sample from each batch of the powders before hot pressing. The powder particle size was analyzed for samples from the first two hot pressed billets, SKD-Wet1 and SKD-Wet2, plus one batch from SKDWet3 (Tables 10.1 and 10.2, Figures 10.1 through 10.3). The particle size measurements were consistent with estimates made via SEM micrographs (Figure 10.4).

Powders of specimens JSpSKD-15 and JSnSKD-14 were pyrophoric when exposed to air, spontaneously combusting while handling. Due to safety concerns, the powder particle size analysis of the milled skutterudite powders was suspended.

Each of the four hot pressed billets was fractured and thermally etched to determine grain size (Table 10.3). The average matrix grain size was consistently less than $2 \mu \mathrm{m}$ in diameter, 
consistent with the powder particle size determined for the wet milled powder (Tables 10.1 and 10.2). Thermally etching, which required a polished specimen surface, was not always successful in revealing the grain boundaries of the etched materials. The only reliable method of grain size analysis was by SEM microscopic analysis of a fractured surface.

Table 10.1. Particle size analysis of the cerium doped N-type skutterudite powder, $\mathrm{Co}_{0.95} \mathrm{Pd}$ ${ }_{0.05} \mathrm{Te}_{0.05} \mathrm{Sb}_{3}$, doped with 0.1 atomic \% Ce, nSKD-Min2. Particle sizing was measured by laser scattering with the Saturn Digitizer in a suspension of $50 \%$ by weight sucrose/ deionized water solution with $0.1 \%$ sodium pyrophosphate as a dispersing liquid.

\begin{tabular}{|c|c|c|c|c|c|c|}
\hline Specimen Name & Type & $\begin{array}{l}\text { Wet or Dry } \\
\text { Milled }\end{array}$ & $\begin{array}{c}\text { Batch } \\
\#\end{array}$ & Test \# & $\begin{array}{c}\text { Median Particle } \\
\text { Size }(\mu \mathrm{m})\end{array}$ & $\begin{array}{c}\text { Mean Particle } \\
\text { Size }(\mu \mathrm{m})\end{array}$ \\
\hline nSKD-Min2 & $\begin{array}{l}\text { Ce-doped } \\
\text { N-type }\end{array}$ & Dry & 2 & 1 & 4.7 & 8.9 \\
\hline nSKD-Min2 & $\begin{array}{l}\text { Ce-doped } \\
\text { N-type }\end{array}$ & Dry & 2 & 2 & 4.3 & 8.8 \\
\hline nSKD-Min2 & $\begin{array}{l}\text { Ce-doped } \\
\text { N-type }\end{array}$ & Dry & 3 & 1 & 3.5 & 9.0 \\
\hline nSKD-Min2 & $\begin{array}{l}\text { Ce-doped } \\
\text { N-type }\end{array}$ & Dry & 3 & 2 & 3.4 & 7.8 \\
\hline nSKD-Min2 & $\begin{array}{l}\text { Ce-doped } \\
\text { N-type }\end{array}$ & Dry & 4 & 1 & 2.6 & 5.6 \\
\hline nSKD-Min2 & $\begin{array}{c}\text { Ce-doped } \\
\text { N-type }\end{array}$ & Wet & 1 & 1 & 2.4 & 5.0 \\
\hline nSKD-Min2 & $\begin{array}{l}\text { Ce-doped } \\
\text { N-type }\end{array}$ & Wet & 2 & 1 & 2.7 & 5.1 \\
\hline nSKD-Min2 & $\begin{array}{l}\text { Ce-doped } \\
\text { N-type }\end{array}$ & Wet & 2 & 2 & 2.7 & 5.0 \\
\hline nSKD-Min2 & $\begin{array}{l}\text { Ce-doped } \\
\text { N-type }\end{array}$ & Wet & 3 & 1 & 2.6 & 5.0 \\
\hline nSKD-Min2 & $\begin{array}{c}\text { Ce-doped } \\
\mathrm{N} \text {-type }\end{array}$ & Wet & 3 & 2 & 2.4 & 3.7 \\
\hline nSKD-Min2 & $\begin{array}{l}\text { Ce-doped } \\
\text { N-type }\end{array}$ & Wet & 3 & 3 & 1.9 & 3.1 \\
\hline nSKD-Min2 & $\begin{array}{l}\text { Ce-doped } \\
\text { N-type }\end{array}$ & Wet & 4 & 1 & 2.0 & 4.1 \\
\hline nSKD-Min2 & $\begin{array}{l}\text { Ce-doped } \\
\text { N-type }\end{array}$ & Wet & 4 & 2 & 1.9 & 3.6 \\
\hline
\end{tabular}


Table 10.2. Particle size analysis of non-cerium doped N-type skutterudite powder, $\mathrm{Co}_{0.95} \mathrm{Pd}_{0.05} \mathrm{Te}_{0.05} \mathrm{Sb}_{3}$, ETN-SKD10. Particle sizing was measured by laser scattering with the Saturn Digisizer in a suspension of $50 \%$ by weight sucrose/ deionized water solution with $0.1 \%$ sodium pyrophosphate as a dispersing liquid.*

\begin{tabular}{|c|c|c|c|c|c|c|}
\hline Specimen Name & Type & $\begin{array}{c}\text { Wet } \\
\text { or Dry } \\
\text { Milled }\end{array}$ & $\begin{array}{c}\text { Batch } \\
\text { Number }\end{array}$ & $\begin{array}{c}\text { Test } \\
\text { Number }\end{array}$ & $\begin{array}{c}\text { Median } \\
\text { Particle } \\
\text { Size ( } \boldsymbol{\mu m})\end{array}$ & $\begin{array}{c}\text { Mean } \\
\text { Particle Size } \\
(\boldsymbol{\mu m})\end{array}$ \\
\hline ETN-SKD10 & N-type & Dry & 1 & 1 & 2.9 & 6.3 \\
\hline ETN-SKD10 & N-type & Dry & 1 & 2 & 3.0 & 7.6 \\
\hline ETN-SKD10 & N-type & Dry & 1 & 3 & 2.9 & 6.7 \\
\hline ETN-SKD10 & N-type & Dry & 2 & 1 & 2.4 & 4.0 \\
\hline ETN-SKD10 & N-type & Dry & 2 & 2 & 2.5 & 4.4 \\
\hline ETN-SKD10 & N-type & Dry & 3 & 1 & 2.8 & 4.9 \\
\hline ETN-SKD10 & N-type & Dry & 3 & 2 & 3.6 & 8.0 \\
\hline ETN-SKD10 & N-type & Dry & 3 & 3 & 3.5 & 7.8 \\
\hline ETN-SKD10 & N-type & Dry & 4 & 1 & 3.1 & 8.1 \\
\hline ETN-SKD10 & N-type & Dry & 4 & 2 & 2.6 & 5.1 \\
\hline ETN-SKD10 & N-type & Dry & 4 & 3 & 2.1 & 3.6 \\
\hline ETN-SKD10* & N-type & Wet & 1 & 1 & 2.1 & 3.8 \\
\hline ETN-SKD10 & N-type & Wet & 1 & 2 & 1.8 & 2.5 \\
\hline ETN-SKD10 & N-type & Wet & 1 & 3 & 1.9 & 2.6 \\
\hline ETN-SKD10 & N-type & Wet & 2 & 1 & 3.2 & 3.4 \\
\hline ETN-SKD10 & N-type & Wet & 2 & 2 & 2.7 & 3.1 \\
\hline ETN-SKD10 & N-type & Wet & 2 & 3 & 1.8 & 3.1 \\
\hline ETN-SKD10 & N-type & Wet & 2 & 4 & 2.0 & 3.9 \\
\hline ETN-SKD10 & N-type & Wet & 2 & 5 & 2.0 & 3.2 \\
\hline ETN-SKD10 & N-type & Wet & 3 & 1 & 2.2 & 4.6 \\
\hline ETN-SKD10 & N-type & Wet & 3 & 2 & 2.6 & 4.0 \\
\hline ETN-SKD10 & N-type & Wet & 3 & 3 & 2.8 & 4.0 \\
\hline ETN-SKD10 & N-type & Wet & 3 & 4 & 2.6 & 4.9 \\
\hline ETN-SKD10 & N-type & Wet & 4 & 1 & 2.6 & 5.2 \\
\hline ETN-SKD10 & N-type & Wet & 4 & 2 & 2.4 & 4.6 \\
\hline
\end{tabular}

*For ETN-SKD10 Wet, Batch 1 Test 1, 0.1\% sodium lignosulfonate was used as the dispersing liquid as a test. Due to agglomeration concerns, this liquid was not used on any other test. 

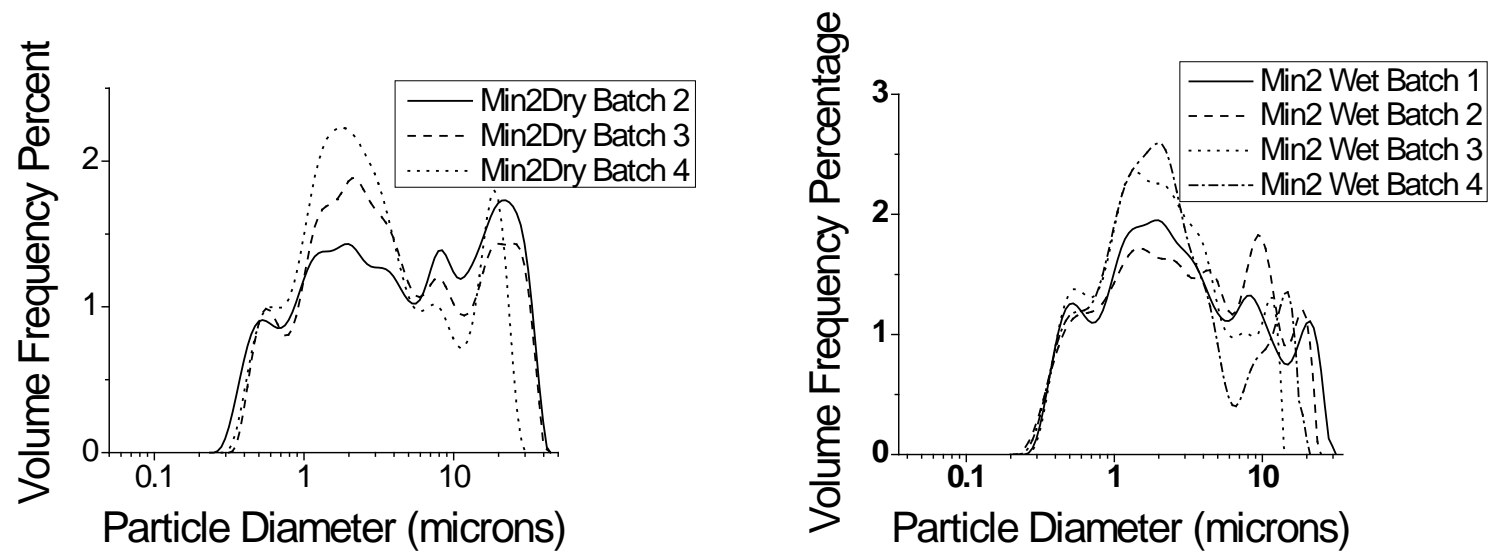

Figure 10.1. Graph of particle size analysis of powders from ingot Min2-SKD, $\mathrm{Co}_{0.95} \mathrm{Pd}_{0.05} \mathrm{Te}_{0.05} \mathrm{Sb}_{3}$, doped with 0.1 atomic \% Ce, from which the wet milled powders were used for specimen SKD-Wet1. The dry milling process produced a powder particle size distribution with two distinct modes at approximately $2 \mu \mathrm{m}$ and $20 \mu \mathrm{m}$. When the dry milled powder was wet milled the average particle size was reduced primarily by reducing the particle size of the larger particles.
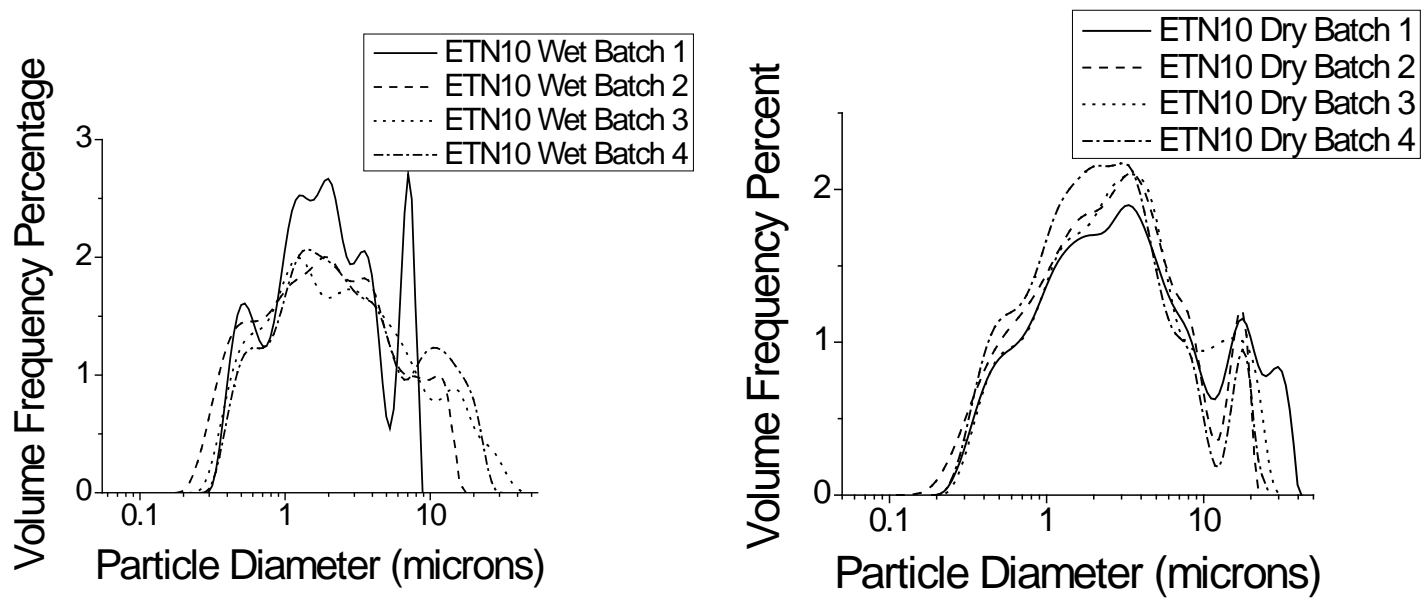

Figure 10.2. Graph of particle size analysis of powders from ingot ETN-SKD-10, $\mathrm{Co}_{0.95} \mathrm{Pd}_{0.05} \mathrm{Te}_{0.05} \mathrm{Sb}_{3}$, from which the wet milled powders were used for specimen SKD-Wet2. The dry milling process produced a powder particle size distribution with two distinct modes at approximately $3 \mu \mathrm{m}$ and $20 \mu \mathrm{m}$. When the dry milled powder was wet milled the average particle size was reduced primarily by reducing the particle size of the larger particles. One representative test from each batch was chosen for graphing for clarity. 


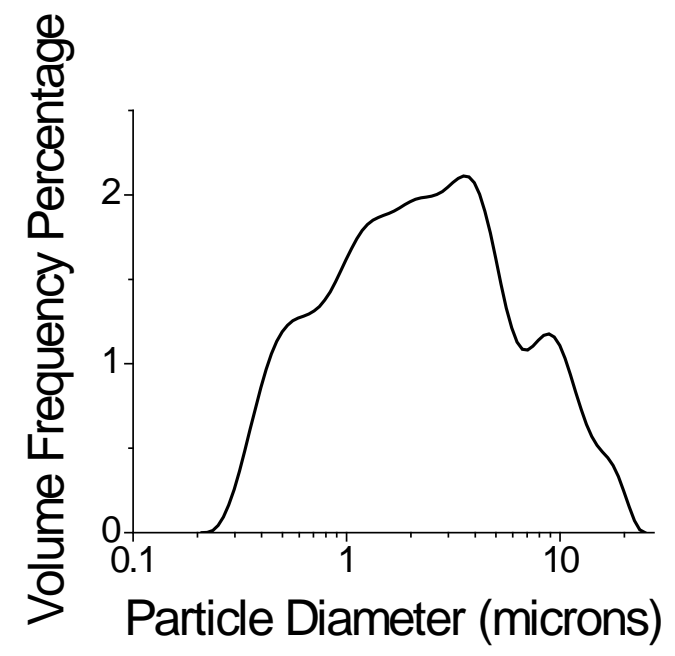

Figure 10.3. Graph of particle size analysis for wet milled powder from ingot JSpSKD-15, $\mathrm{Ce}_{0.9} \mathrm{Fe}_{3.5} \mathrm{Co}_{0.5} \mathrm{Sb}_{12}$, Batch 2, one of 4 batches of milled powder from the same ingot used to make SKD-Wet3. Due to the second specimen showing pyrophoric behavior before it could be analyzed, this was the only batch analyzed. The hot pressed billet had a bimodal distribution, with a matrix of an average $1.2 \mu \mathrm{m}$ diameter with grains interspersed of over $5 \mu \mathrm{m}$ diameter. This bimodal character was not evident in the powder particle size distribution to the extent observed in the hot pressed specimens.

The limited sample size of one prevented further investigation.
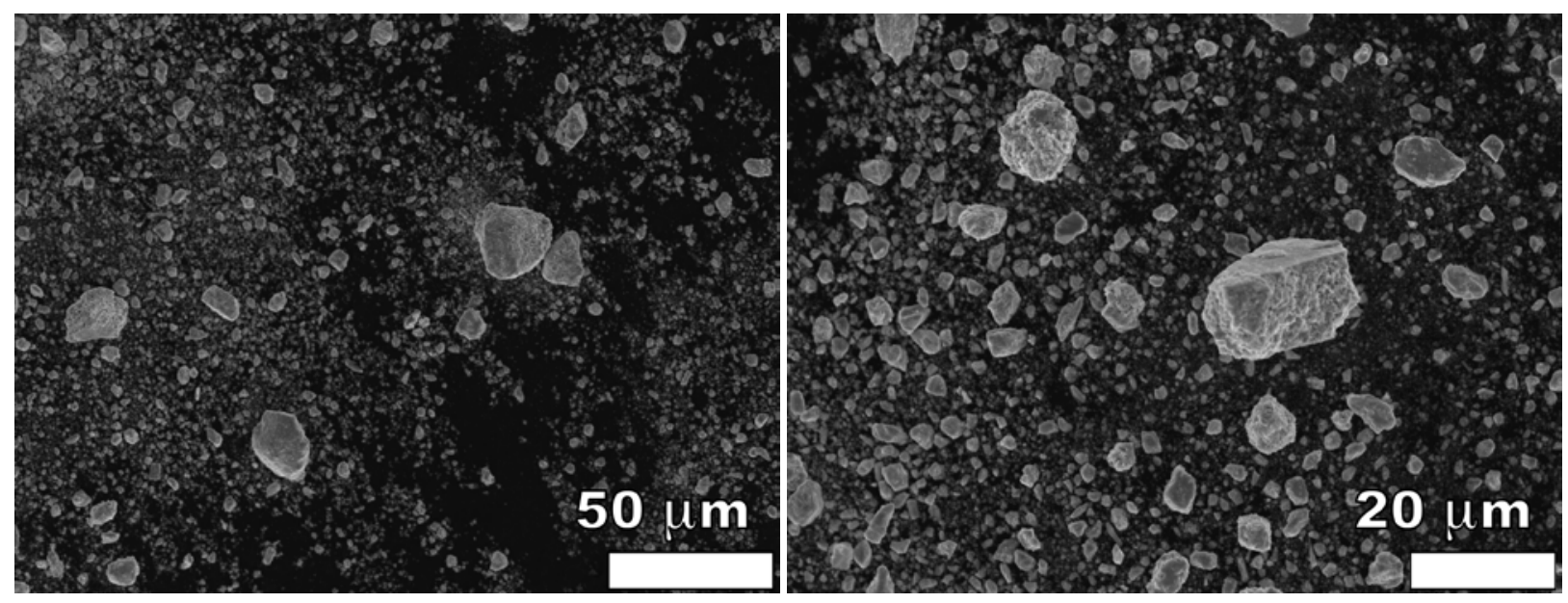

Figure 10.4. Micrographs of dry milled (50 $\mu \mathrm{m}$ scale) and wet milled ( $20 \mu \mathrm{m}$ scale) powders from nSKD-Min2 Batch 1, $\mathrm{Co}_{0.95} \mathrm{Pd}_{0.05} \mathrm{Te}_{0.05} \mathrm{Sb}_{3}$, doped with 0.1 atomic \% Ce, for powder shape evaluation and qualitative comparison with laser particle size analysis. The sizes of the particles observed by SEM are consistent with the laser particle size analysis.

Note the length scale change between the dry milled and the wet milled powder. 
Table 10.3. Grain size for all wet milled specimens was observed to be below $2 \mu \mathrm{m}$ for the matrix. The P-type specimen, SKD-Wet3, composition $\mathrm{Ce}_{0.9} \mathrm{Fe}_{3.5} \mathrm{Co}_{0.5} \mathrm{Sb}_{12}$, exhibited bimodal structure with some larger grains of 5-10 $\mu \mathrm{m}$ within the matrix.

Given the grain sizes observed, any grain size distribution between the wet milled specimens will be limited to the range of 1-2 $\mu \mathrm{m}$.

\begin{tabular}{|c|c|c|c|}
\hline $\begin{array}{c}\text { Billet } \\
\text { Label }\end{array}$ & Type & $\begin{array}{c}\text { Grain Size via } \\
\text { Fracture }(\boldsymbol{\mu m})\end{array}$ & $\begin{array}{c}\text { Grain Size via Thermal Etch } \\
(\boldsymbol{\mu m})\end{array}$ \\
\hline $\begin{array}{c}\text { SKD- } \\
\text { Wet1 }\end{array}$ & N-type, Ce-doped & $0.9-1.0^{\mathrm{a}}$ & $\mathrm{NA}^{\mathrm{b}}$ \\
\hline $\begin{array}{c}\text { SKD- } \\
\text { Wet2 }\end{array}$ & N-type & 1.5 & $1.3-1.7^{\mathrm{a}}$ \\
\hline $\begin{array}{c}\text { SKD- } \\
\text { Wet3 }\end{array}$ & P-type & $1.2^{\mathrm{c}}$ & $\mathrm{NA}^{\mathrm{b}}$ \\
\hline $\begin{array}{c}\text { SKD- } \\
\text { Wet4 }\end{array}$ & N-type & $1.7-2.0^{\mathrm{a}}$ & $1.1-1.5^{\mathrm{a}}$ \\
\hline $\begin{array}{c}\text { SKD- } \\
\text { 31-9 }\end{array}$ & N-type & $\mathrm{NA}^{\mathrm{b}}$ & $1.8-2.8^{\mathrm{a}}$ \\
\hline
\end{tabular}

${ }^{a}$ Range is provided when more than one SEM micrograph of the same specimen was analyzed.

${ }^{b}$ NA - Not Available. For SKD-Wet1 and SKD-Wet3, thermal etch was unsuccessful. For SKD-31-9, further analysis was not run due to limited specimen availability and limited processing information.

${ }^{\mathrm{c}} \mathrm{SKD}-W e t 3$ exhibited bimodal grain sizes, with grains of 5-10 $\mu \mathrm{m}$ interspersed within the $1.2 \mu \mathrm{m}$ matrix.

\subsection{Elastic Modulus Measurements}

The room temperature elastic moduli were measured by (i) resonant ultrasound spectroscopy and (ii) nanoindentation. Specimens for RUS were nominally rectangular parallelepipeds 10 x 7 × 5 $\mathrm{mm}$. The Young's moduli for P-type specimens were 126.8 to $131.2 \mathrm{GPa}$, the shear modulus was 51.04 to $53.35 \mathrm{GPa}$ and Poisson's ratio was 0.230 to 0.242 (Table 10.4). For N-type skutterudite, the Young's modulus was 129.0 to $140.6 \mathrm{GPa}$, shear modulus was 52.28 to 57.34 GPa, and Poisson's ratio was 0.226 to 0.234 .

The moduli measured by RUS were consistent within each billet to within a maximum standard deviation of 1.2 GPa for Young's modulus, 0.54 GPa for shear modulus and 0.011 for Poisson's ratio. The variation in moduli may be accounted for, at least in part, by a variation in specimen porosity. In addition, in the case of the N-type skutterudite material, the addition of 0.1 atomic \% cerium in SKD-Wet1 may account for some variation in moduli between SKD-Wet1 and the other two N-type materials, SKD-Wet2 and SKD-Wet4 (Figures 10.5 and 10.6). The elastic modulus data were fit to equation (1),

$A=A_{0} \exp (-b P)$

where A was the Young's or shear modulus at the indicated porosity, $A_{0}$ was the theoretically dense Young's or shear modulus, $\mathrm{P}$ was the volume fraction porosity, and $\mathrm{b}$ was a materialdependent fitting parameter (Table 10.5). 


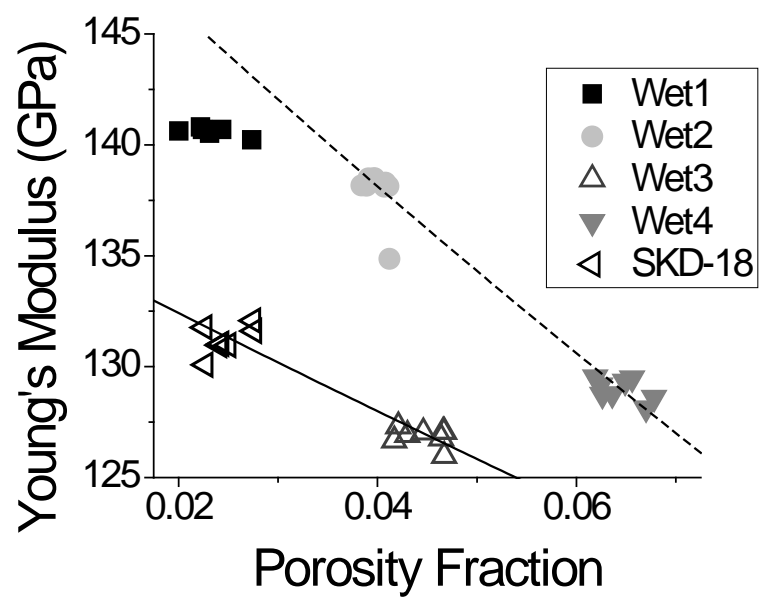

Figure 10.5. The porosity dependence of Young's modulus. The porosity dependence of the P-type skutterudite (open symbols) and N-type skutterudite without cerium dopant is shown with the porosity relation fitted. Note SKD-Wet1, the N-type with cerium dopant (filled square), falls below the relationship line for the non-cerium doped specimens.

Table 10.4. Elastic moduli data for the wet milled billets and one production billet, averaged from multiple specimens tested. The specimens exhibited some differences based on porosity and composition effects. The porosity relationship may be observed between

SKD-Wet2 and SKD-Wet4, both of composition, as well as between SKD-Wet3 and SKD-18, both of composition $\mathrm{Ce}_{0.9} \mathrm{Fe}_{3.5} \mathrm{Co}_{0.5} \mathrm{Sb}_{12}$, as each pair may be examined together because they are the same composition.

\begin{tabular}{|l|c|c|c|c|c|c|}
\hline Billet Label & $\begin{array}{c}\text { Number of } \\
\text { Specimens }\end{array}$ & Type & Relative Density & E (GPa) & G (GPa) & v \\
\hline SKD-Wet1 & 6 & N-type, & $0.977 \pm .002$ & $140.6 \pm$ & $57.34 \pm$ & 0.226 \\
& & Ce-doped & & 0.2 & 0.05 & \pm .001 \\
\hline SKD-Wet2 & 8 & N-type & $0.960 \pm .001$ & $137.8 \pm$ & $56.01 \pm$ & 0.231 \\
& & & & 1.2 & 0.26 & \pm .006 \\
\hline SKD-Wet3 & 8 & P-type & $0.956 \pm .002$ & $126.8 \pm$ & $51.04 \pm$ & 0.242 \\
& & & & 0.4 & 0.30 & \pm .011 \\
\hline SKD-Wet4 & 8 & N-type & $0.936 \pm .002$ & $129.0 \pm$ & $52.28 \pm$ & 0.234 \\
& & & & 0.5 & 0.39 & \pm .008 \\
\hline SKD-18 & 7 & P-type & $0.975 \pm .002$ & $131.2 \pm$ & $53.35 \pm$ & 0.230 \\
& & & & 0.6 & 0.54 & \pm .008 \\
\hline
\end{tabular}




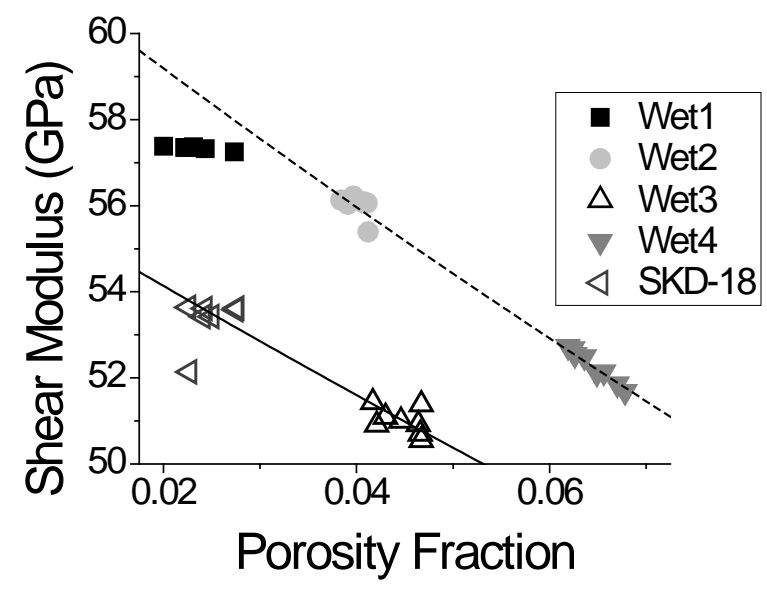

Figure 10.6. The porosity dependence of the shear modulus. The porosity dependence of the Ptype skutterudite, $\mathrm{Ce}_{0.9} \mathrm{Fe}_{3.5} \mathrm{Co}_{0.5} \mathrm{Sb}_{12}$, (open symbols) and $\mathrm{N}$-type skutterudite without cerium dopant is shown with the porosity relation fitted. Note SKD-Wet1, the N-type with cerium dopant (filled square), falls below the relationship line for the non-cerium doped specimens.

Table 10.5. Porosity relationship parameters for the skutterudite materials. These parameters are the fitting parameters for the elastic moduli to porosity relationship equation, $A=A_{0} \exp (-$ $\mathrm{bP}$ ), where $A$ is the parameter to be fitted (Young's modulus, E, or shear modulus, $G$ ), $A_{0}$ is the theoretically dense value for the modulus, $\mathrm{P}$ is the volume percent porosity, and $\mathrm{b}$ is an experimentally determined fitting constant [12].

\begin{tabular}{|l|l|l|}
\hline \multicolumn{1}{|c|}{ Parameters } & $\begin{array}{l}\text { Non-cerium Doped } \\
\text { N-type Constants }\end{array}$ & \multicolumn{1}{c|}{$\begin{array}{c}\text { P-type } \\
\text { Constants }\end{array}$} \\
\hline $\mathrm{E}_{0}$ & $154.5 \mathrm{GPa}$ & $137 \mathrm{GPa}$ \\
\hline $\mathrm{b}(\mathrm{E})$ & 2.8 & 1.7 \\
\hline $\mathrm{G}_{0}$ & $62.6 \mathrm{GPa}$ & $56.8 \mathrm{GPa}$ \\
\hline $\mathrm{b}(\mathrm{G})$ & 2.8 & 2.4 \\
\hline
\end{tabular}

Specimens of SKD-Wet1 and SKD-Wet2 were polished on 3 mutually orthogonal faces, one face normal to the hot press direction, and nanoindented on each face (Table 10.6). The results of a nanoindentation study indicated no significant anisotropy for either specimen SKD-Wet1 or SKD-Wet2. The Young's modulus of SKD-Wet1 was $149.8 \mathrm{GPa}$ and $154.7 \mathrm{GPa}$ and the Young's modulus of SKD-Wet2 was from 139.4 GPa and 151.0 GPa [13]. The measured values of the Young's moduli (Table 10.6) were between the theoretically dense and the measured bulk values, as anticipated. 
Table 10.6. Nanoindentation results for Young's moduli. For all specimens, Poisson's ratio was assumed as indicated to allow calculation of Young's modulus on unloading. For SKD-Wet1 and SKD-Wet2, three mutually orthogonal sides were indented to verify the specimens as isotropic, regardless of the direction of pressure during hot pressing.

No significant anisotropy was noted. Before each batch of indentations were attempted, a standard aluminum specimen was indented to verify the consistency of the measurement and as a method to clean the indenter tip of debris. Results for SKD-Wet1, SKD-Wet2 and aluminum previously reported by Schmidt et al. [13].

\begin{tabular}{|c|c|c|c|c|c|c|c|}
\hline $\begin{array}{l}\text { Billet } \\
\text { Label }\end{array}$ & $\begin{array}{c}\text { Number of } \\
\text { Indentations }\end{array}$ & Type & $\begin{array}{l}\text { Density } \\
\left(\mathrm{g} / \mathrm{cm}^{3}\right)\end{array}$ & $\begin{array}{c}\text { Relative } \\
\text { density }\end{array}$ & $\begin{array}{c}\text { In pressing } \\
\text { direction }\end{array}$ & E (GPa) & $v$ \\
\hline $\begin{array}{l}\text { SKD- } \\
\text { Wet1 }\end{array}$ & 22 & N-type & 7.59 & 0.977 & No & $\begin{array}{c}154.7 \pm \\
1.2\end{array}$ & 0.23 \\
\hline $\begin{array}{l}\text { SKD- } \\
\text { Wet1 }\end{array}$ & 20 & N-type & 7.59 & 0.977 & No & $\begin{array}{c}153.1 \pm \\
1.9\end{array}$ & 0.23 \\
\hline $\begin{array}{l}\text { SKD- } \\
\text { Wet1 }\end{array}$ & 21 & N-type & 7.59 & 0.977 & Yes & $\begin{array}{c}149.8 \pm \\
2.2\end{array}$ & 0.23 \\
\hline $\begin{array}{l}\text { SKD- } \\
\text { Wet2 }\end{array}$ & 18 & N-type & 7.45 & 0.960 & No & $\begin{array}{c}151.0 \pm \\
2.6 \\
\end{array}$ & 0.23 \\
\hline $\begin{array}{l}\text { SKD- } \\
\text { Wet2 }\end{array}$ & 16 & N-type & 7.45 & 0.960 & No & $\begin{array}{c}147.5 \pm \\
3.2\end{array}$ & 0.23 \\
\hline $\begin{array}{l}\text { SKD- } \\
\text { Wet2 }\end{array}$ & 20 & N-type & 7.45 & 0.960 & Yes & $\begin{array}{c}139.4 \pm \\
2.5\end{array}$ & 0.23 \\
\hline $\begin{array}{c}\text { Al } 6061 \\
\text { Test } 1\end{array}$ & 16 & Metal & 2.69 & 0.998 & $\begin{array}{c}\text { Not } \\
\text { applicable }\end{array}$ & $81.0 \pm 2.1$ & 0.33 \\
\hline $\begin{array}{c}\text { Al } 6061 \\
\text { Test } 2\end{array}$ & 16 & Metal & 2.69 & 0.998 & $\begin{array}{c}\text { Not } \\
\text { applicable }\end{array}$ & $81.9 \pm 1.8$ & 0.33 \\
\hline $\begin{array}{c}\text { Al } 6061 \\
\text { Test } 3\end{array}$ & 14 & Metal & 2.69 & 0.998 & $\begin{array}{c}\text { Not } \\
\text { applicable }\end{array}$ & $82.0 \pm 2.0$ & 0.33 \\
\hline $\begin{array}{c}\text { Al } 6061 \\
\text { Test } 4\end{array}$ & 15 & Metal & 2.69 & 0.998 & $\begin{array}{c}\text { Not } \\
\text { applicable }\end{array}$ & $83.3 \pm 2.4$ & 0.33 \\
\hline
\end{tabular}

\subsection{Vickers Hardness Results}

Vickers hardness for each specimen was determined for multiple loads between $0.245 \mathrm{~N}$ and 4.9 $\mathrm{N}$ (Table 10.7). No statistically significant trend in hardness as a function of load was observed since all the error bars overlapped (Figure 10.7). However, for the N-type material, a possible indentation size effect was observed for loads less than $1 \mathrm{~N}$ (Figure 10.7). The measured hardness was between 4.35 GPa and 7.04 GPa for all specimens, with the hardness increasing with the density of the specimen (Tables 10.4 and 10.7).

\subsection{Thermal Expansion Results}

Using a Thermomechanical Analyzer (TMA, Q400, TA Instruments, New Castle, DE) the thermal expansion was measured for bulk specimens cut from each of four wet milled billets (Table 10.8). The N-type, cerium doped specimen had a thermal expansion coefficient of $11.6 \mathrm{x}$ $10^{-6} \mathrm{~K}^{-1}$; for the non-cerium doped, the CTE was 9.8 to $10.2 \times 10^{-6} \mathrm{~K}^{-1}$. For the P-type specimen, the thermal expansion coefficient was $13.0 \times 10^{-6} \mathrm{~K}^{-1}$. Via X-ray powder diffraction for the Ptype specimen, a CTE of $13.0 \times 10^{-6} \mathrm{~K}^{-1}$ was measured. 
The lattice parameter of the P-type material was measured by X-ray diffraction as 9.1241 angstroms at $303 \mathrm{~K}$, and increased linearly through $633 \mathrm{~K}$ (Figure 10.8, Table 10.9). A new set of X-ray diffraction peaks appeared on the XRD pattern when the powder was heated to $603 \mathrm{~K}$. The extra XRD peaks indicate a new crystallographic phase developed around $603 \mathrm{~K}$ and remained stable as the sample was cooled to room temperature (Figure 10.9).

Table 10.7. Vickers Hardness Measurements. Some Vickers indentation measurements were difficult to measure optically and were measured from SEM imaging, as indicated.

\begin{tabular}{|c|c|c|c|c|c|c|}
\hline Specimen & Type & Load (N) & $\begin{array}{c}\text { Measurement } \\
\text { Technique } \\
\end{array}$ & $\begin{array}{c}\text { Hardness } \\
\text { (GPa) }\end{array}$ & Standard Dev & COV \\
\hline $\begin{array}{c}\text { SKD- } \\
\text { Wet1-G }\end{array}$ & N-type & 4.9 & Optical & 6.62 & 0.30 & 0.04 \\
\hline $\begin{array}{c}\text { SKD- } \\
\text { Wet1-H }\end{array}$ & N-type & 2.94 & Optical & 6.42 & 0.17 & 0.03 \\
\hline $\begin{array}{c}\text { SKD- } \\
\text { Wet1-J }\end{array}$ & N-type & 0.98 & Optical & 6.66 & 0.40 & 0.06 \\
\hline $\begin{array}{c}\text { SKD- } \\
\text { Wet1-J }\end{array}$ & N-type & 0.49 & Optical & 6.95 & 0.50 & 0.07 \\
\hline $\begin{array}{c}\text { SKD- } \\
\text { Wet1-J }\end{array}$ & N-type & 0.245 & Optical & 7.04 & 0.41 & 0.06 \\
\hline $\begin{array}{c}\text { SKD- } \\
\text { Wet2-K }\end{array}$ & N-type & 0.98 & Optical & 5.36 & 0.28 & 0.05 \\
\hline $\begin{array}{c}\text { SKD- } \\
\text { Wet2-K }\end{array}$ & N-type & 0.49 & SEM & 5.67 & 0.29 & 0.05 \\
\hline $\begin{array}{c}\text { SKD- } \\
\text { Wet2-K }\end{array}$ & N-type & 0.245 & SEM & 5.92 & 0.29 & 0.05 \\
\hline $\begin{array}{c}\text { SKD- } \\
\text { Wet3-A }\end{array}$ & P-type & 0.98 & Optical & 5.12 & 0.30 & 0.06 \\
\hline $\begin{array}{c}\text { SKD- } \\
\text { Wet3-A }\end{array}$ & P-type & 0.98 & SEM & 4.83 & 0.20 & 0.04 \\
\hline $\begin{array}{c}\text { SKD- } \\
\text { Wet3-A }\end{array}$ & P-type & 0.49 & Optical & 5.68 & 1.74 & 0.31 \\
\hline $\begin{array}{c}\text { SKD- } \\
\text { Wet3-A }\end{array}$ & P-type & 0.49 & SEM & 5.67 & 0.29 & 0.05 \\
\hline $\begin{array}{c}\text { SKD- } \\
\text { Wet3-A }\end{array}$ & P-type & 0.245 & Optical & 5.17 & 0.33 & 0.06 \\
\hline $\begin{array}{c}\text { SKD- } \\
\text { Wet4-G }\end{array}$ & N-type & 0.98 & Optical & 4.35 & 0.33 & 0.08 \\
\hline $\begin{array}{c}\text { SKD- } \\
\text { Wet4-G }\end{array}$ & N-type & 0.49 & Optical & 4.58 & 0.32 & 0.07 \\
\hline $\begin{array}{c}\text { SKD- } \\
\text { Wet4-G }\end{array}$ & N-type & 0.245 & Optical & 4.86 & 0.44 & 0.09 \\
\hline SKD-17 & P-type & 2.94 & Optical & 5.63 & 0.24 & 0.04 \\
\hline SKD-18 & P-type & 2.94 & Optical & 5.60 & 0.21 & 0.04 \\
\hline
\end{tabular}



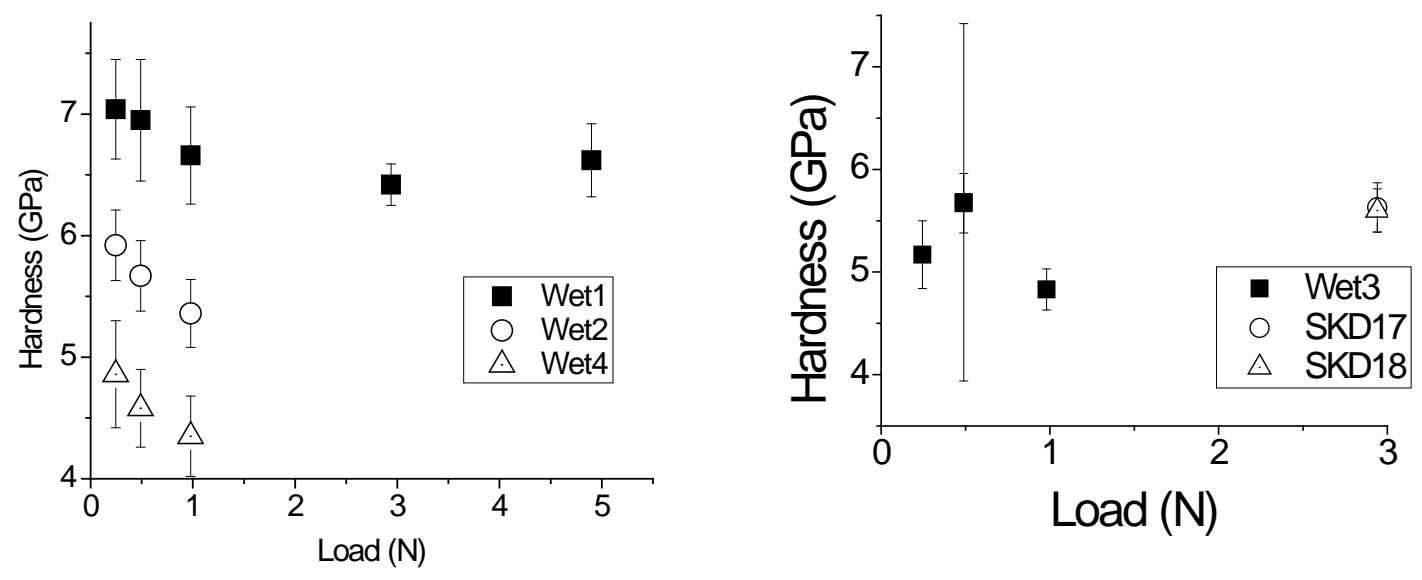

Figure 10.7. Vickers hardness measurements for N-type (a) and P-type (b). The N-type hardness exhibited consistent hardness values, with a possible rise in hardness at lower loads. Increased hardness corresponds to decreasing porosity between billets. The P-type specimens were less consistent, but all were in a range of 4.8 to $5.7 \mathrm{GPa}$, regardless of load. The plot shows the difference in error between two measuring techniques at the same load, with measurement by SEM micrographs producing a smaller error on the same indentations than optical measurement.

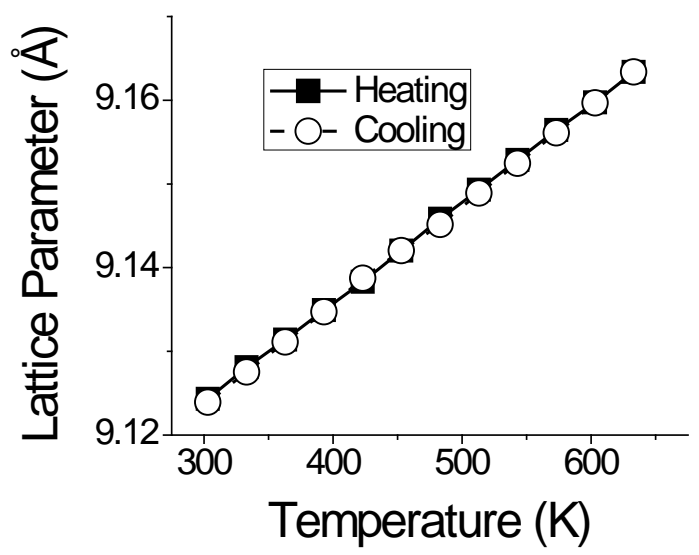

Figure 10.8. Plot of lattice parameter for P-type powder JSpSKD-15 composition $\mathrm{Ce}_{0.9} \mathrm{Fe}_{3.5} \mathrm{Co}_{0.5} \mathrm{Sb}_{12}$ from the powder hot pressed to make SKD-Wet3. The heating and cooling were very linear and consistent despite the new peaks forming between $573 \mathrm{~K}$ and $603 \mathrm{~K}$ on heating and remaining during the entire cooling cycle. 
Table 10.9. Lattice Parameter Measurement of P-type Skutterudite powder specimen JSP-SKD-15, composition $\mathrm{Ce}_{0.9} \mathrm{Fe}_{3.5} \mathrm{Co}_{0.5} \mathrm{Sb}_{12}$. The powder was a sample of the material later pressed into P-SKD-Wet3.

\begin{tabular}{|c|c|c|}
\hline $\begin{array}{c}\text { Temperature } \\
(\mathbf{K})\end{array}$ & $\begin{array}{c}\text { Lattice Parameter } \\
\text { During Heating }(\boldsymbol{\AA})\end{array}$ & $\begin{array}{c}\text { Lattice Parameter } \\
\text { During Cooling }(\AA)\end{array}$ \\
\hline 303 & 9.1243 & 9.1239 \\
\hline 333 & 9.1281 & 9.1275 \\
\hline 363 & 9.1314 & 9.1311 \\
\hline 393 & 9.1349 & 9.1347 \\
\hline 423 & 9.1383 & 9.1387 \\
\hline 453 & 9.1420 & 9.1420 \\
\hline 483 & 9.1458 & 9.1451 \\
\hline 513 & 9.1493 & 9.1489 \\
\hline 543 & 9.1529 & 9.1525 \\
\hline 573 & 9.1564 & 9.1561 \\
\hline 603 & 9.1597 & 9.1597 \\
\hline 633 & $9.1634^{*}$ & $9.1634^{*}$ \\
\hline
\end{tabular}

*The $633 \mathrm{~K}$ lattice parameter was measured once as the maximum temperature. There was no separate heating and cooling measurement for $633 \mathrm{~K}$.

The elastic moduli decreased linearly with the increasing temperature over the interval from room temperature to a cutoff temperature between $523 \mathrm{~K}$ and $623 \mathrm{~K}$. At temperatures greater than the cutoff temperature (Figure 10.10 through Figure 10.13) a viscoelastic region began and the elastic moduli decreased nonlinearly (Figure 10.10 through Figure 10.13). The acoustic Debye temperature $\theta_{\mathrm{D}}$ (Table 10.10) was between $303 \mathrm{~K}$ and $318 \mathrm{~K}$ for all of the N-type and Ptype skutterudite specimens. The typical high temperature limit for cryogenic behavior (Figure 10.13) of $0.3 \theta_{\mathrm{D}}$ to $0.5 \theta_{\mathrm{D}}$ [14] was well below the range of test temperatures included in this study.

The skutterudite thermoelectric materials in this study are sturdy enough to be assembled into a thermoelectric generator for a temperature range from room temperature to $603 \mathrm{~K}$. As discussed above, at $603 \mathrm{~K}$ a new phase developed in the P-type material. Also, between $573 \mathrm{~K}$ and $773 \mathrm{~K}$, an unidentified rust-colored surface layer developed on the P-type during high temperature coefficient of thermal expansion measurements. The thickness of the rust-colored coating was extremely thin, however, since as determined by mass measurements using an electronic balance before and after the CTE measurements, the mass of the P-type skutterudite changed by not more than $10^{-4}$ gram.

The apparent phase changes and/or reactions that occur in the P-type skutterudite materials range of approximately 573 to $603 \mathrm{~K}$ suggest that the P-type material should be studied further above $603 \mathrm{~K}$ to determine if the material remains suitable for use. In addition all specimens reached a viscoelastic region above $623 \mathrm{~K}$ (Figures 10.10, 10.11 and 10.13). This may reduce the mechanical integrity of a thermoelectric device if operated above $623 \mathrm{~K}$.

The CTE of the P-type skutterudite material was approximately 30\% higher than the N-type, non-cerium doped material. The increased thermal expansion needs to be managed in a device. In general the skutterudite materials in this study may be used between room temperature and 
$603 \mathrm{~K}$ without material changes. Use at temperatures higher than $603 \mathrm{~K}$ may require further study.

\subsection{Future Mechanical Characterization Work}

The porosity-elastic moduli relationship has been determined for a narrow range between 0.023 and 0.064 volume fraction porosity for the P-type skutterudite material and the non-cerium doped N-type material (Figures 10.6 and 10.7 and Table 10.5). With the narrow range of experimental porosity values, it is not feasible to accurately determine the elastic modulusporosity relationship. Also, the porosity-elastic modulus relationship for cerium-doped N-type skutterudite material has not been evaluated. Additional specimens of all of the skutterudite materials in this study with varying volume fraction porosity could be used to evaluate and extend the porosity-elastic moduli relationships found in this study.

If the skutterudite materials are used at temperatures above $623 \mathrm{~K}$, the viscoelastic region of the temperature-elastic moduli region (Figure 10.10 through Figure 10.13) must be examined further, especially for the P-type skutterudites (Figure 10.12). For each of the skutterudite materials included in this study, the mechanism for the viscoelastic behavior should be determined to ensure the material is stable above $623 \mathrm{~K}$. X-ray diffraction studies of the P-type skutterudite material showed a new minor phase developed in XRD at $603 \mathrm{~K}$, which should be identified, and the P-type material checked for mechanical, electrical and thermal properties after heating above $603 \mathrm{~K}$.

An unidentified rust-colored surface layer developed on the P-type skutterudite material when heated to $773 \mathrm{~K}$ in a $96 \%$ argon $4 \%$ hydrogen atmosphere. The nature and physical properties of the rust-colored surface layer should be determined.

For all specimens included in this study, the thermal expansion curves showed an irreversible increase in specimen volume that occurred at temperatures above $573 \mathrm{~K}$ (Figures 10.15 through 10.18). Although the physical mechanism that generates the irreversible increase in the specimen volume has not yet been identified, understanding the behavior may be necessary to use the skutterudite materials above $600 \mathrm{~K}$.

Additional measurements of the Vickers hardness would be enlightening to see if there is an indentation size effect in the N-type material for loads of less than $1 \mathrm{~N}$. Current results were inconclusive due to the amount of error in the hardness measurement, but suggested a possible indentation size effect on the N-type material. 


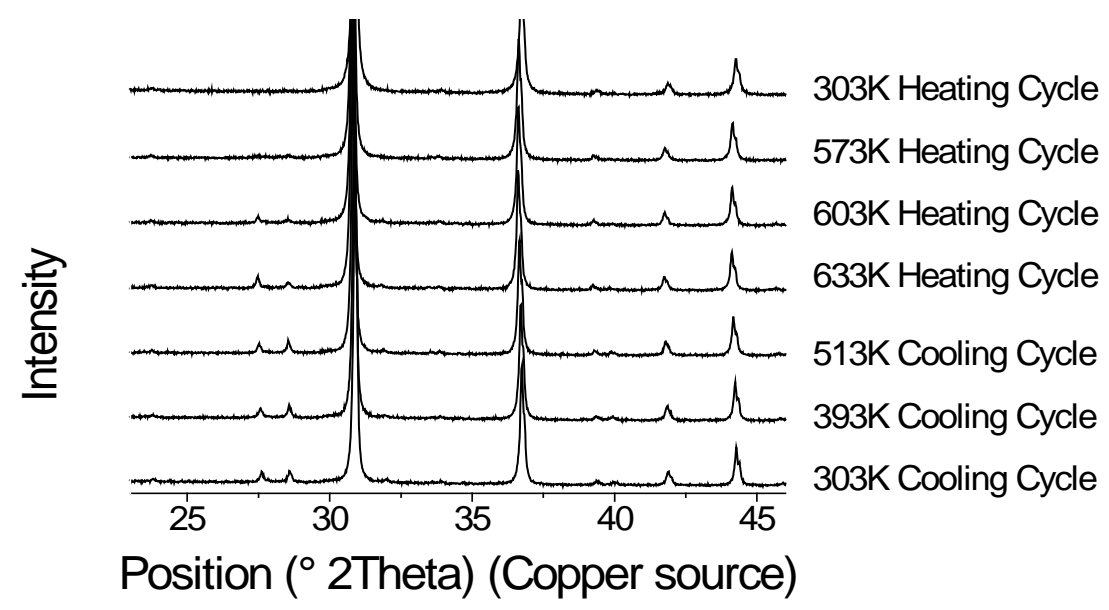

Figure 10.9. The x-ray diffraction charts of powders of JSpSKD-15, composition $\mathrm{Ce}_{0.9} \mathrm{Fe}_{3.5} \mathrm{Co}_{0.5} \mathrm{Sb}_{12}$, show the development of two new peaks between $27^{\circ}$ and $29^{\circ}$ that were not detected until heating above $573 \mathrm{~K}$. The earliest measurement is at the top and selected measurements in chronological order are visible as moving down. The peaks were not visible until after heating to $573 \mathrm{~K}$, indicating a new phase was growing, beginning around $573 \mathrm{~K}$. The peaks remained when the specimen was cooled back below 573K, remaining unchanged through all the cooling cycle measurements. 

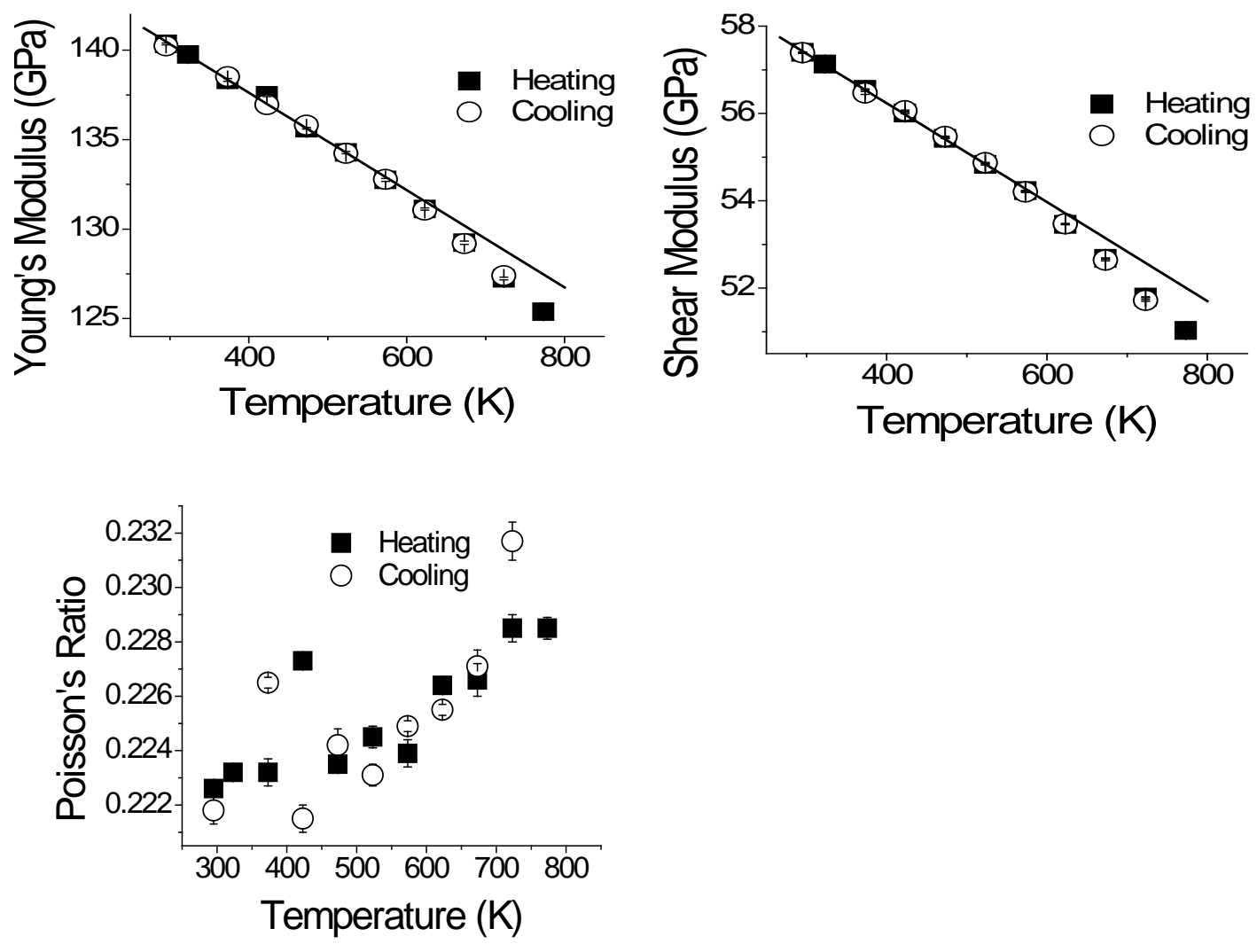

Figure 10.10. The Young's modulus and shear modulus of SKD-Wet1, a and b, changed linearly with temperature from room temperature until above $600 \mathrm{~K}$. Similarly, the Poisson's ratio, $v$, remained relatively constant around 0.224 until approximately $600 \mathrm{~K}$, above which it began to rise. 

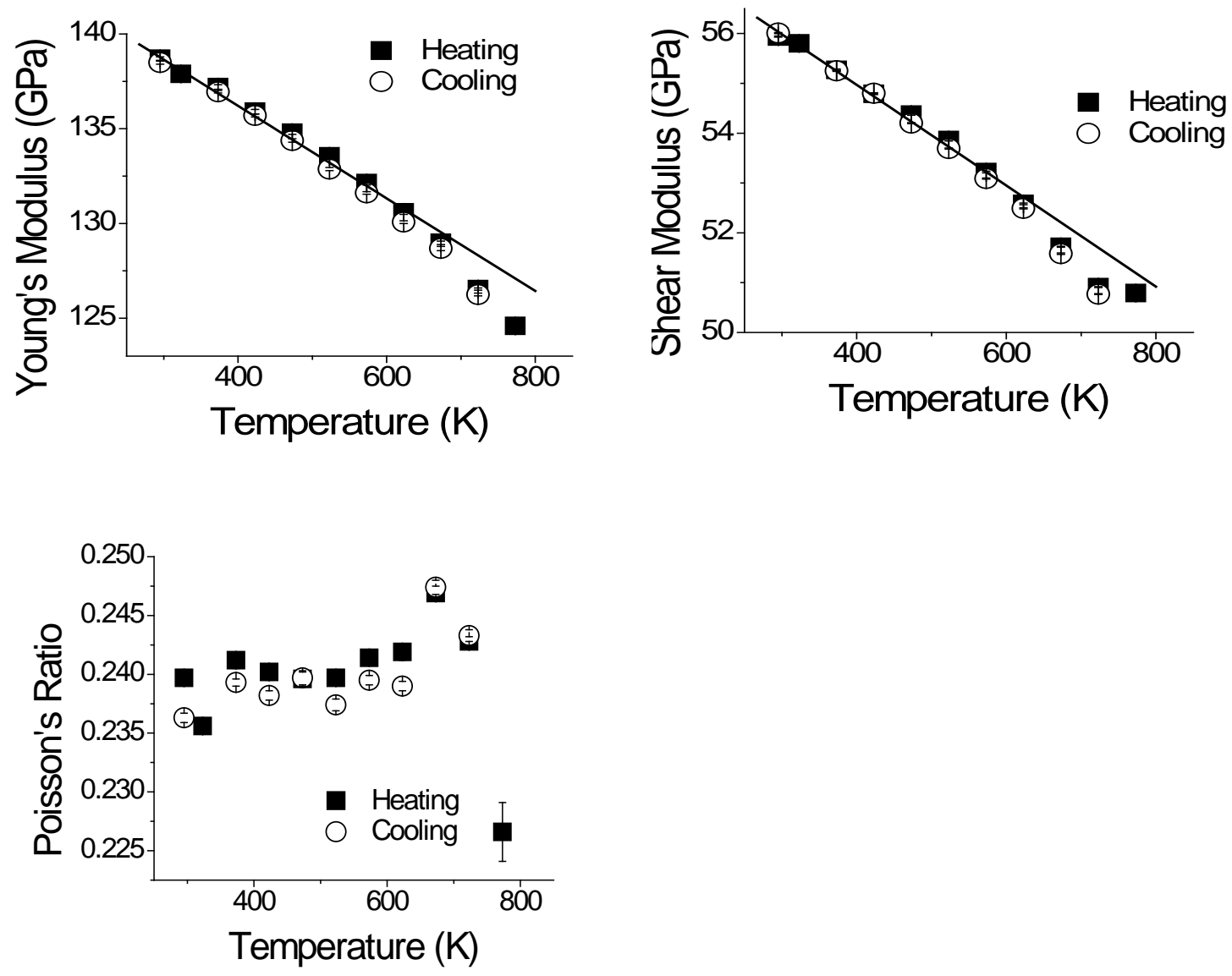

Figure 10.11. The Young's modulus and shear modulus of SKD-Wet2, a and b, changed linearly with temperature from room temperature until above $600 \mathrm{~K}$. The highest temperature datapoint, $773 \mathrm{~K}$, was an outlier, although the material returned to a similar measured value of all elastic moduli during cooling. Similarly, the Poisson's ratio, c, remained relatively constant around 0.240 until above $623 \mathrm{~K}$, above which it began to rise with an outlier at $773 \mathrm{~K}$. 

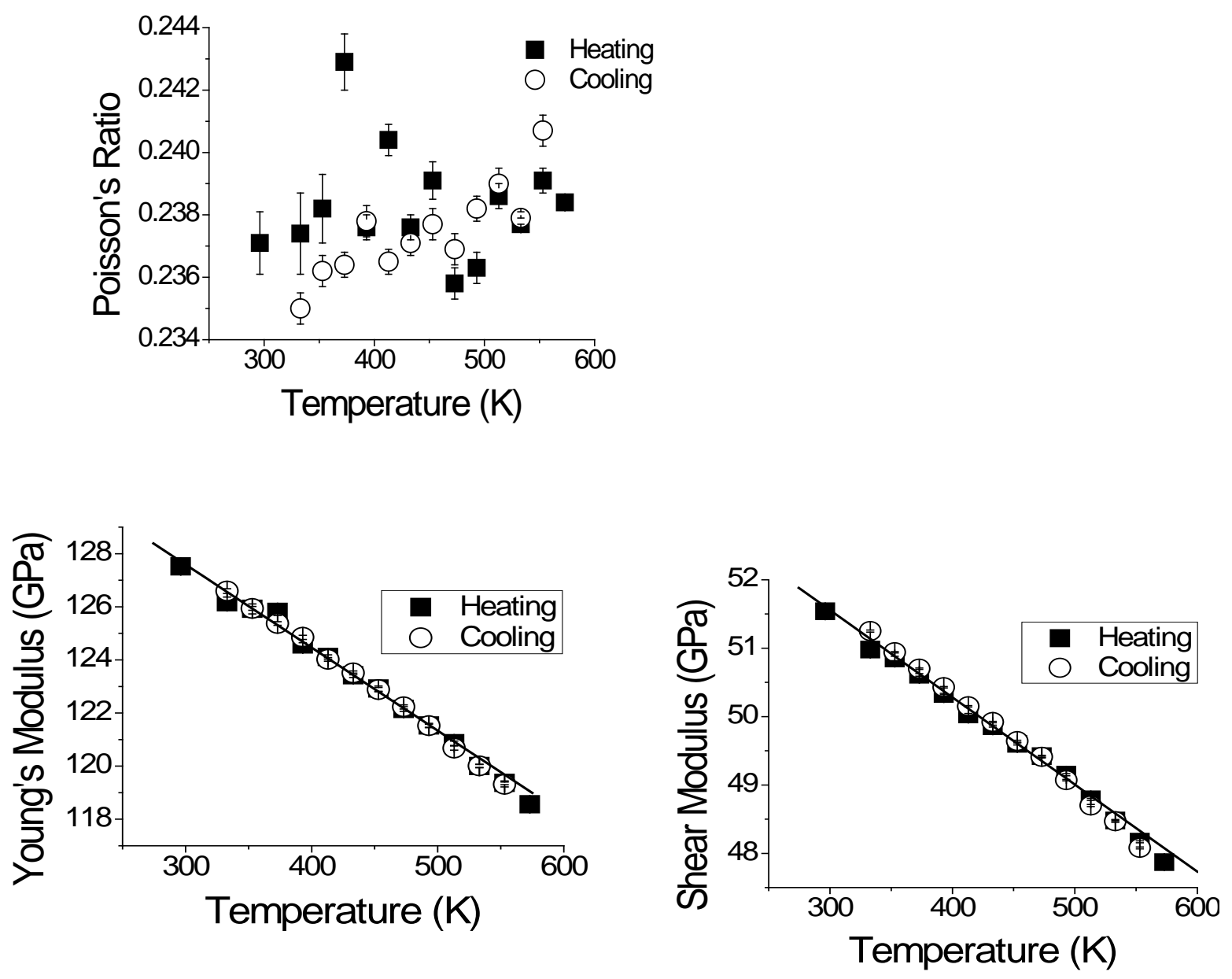

Figure 10.12. The Young's and shear moduli of SKD-Wet3, composition $\mathrm{Ce}_{0.9} \mathrm{Fe}_{3.5} \mathrm{Co}_{0.5} \mathrm{Sb}_{12}$, remained linear with temperature until above $523 \mathrm{~K}$, when it began to increase the reduction of moduli with temperature. Some scatter exists within the Poisson's ratio, although it remains fairly constant near 0.238 throughout the range of $298 \mathrm{~K}$ to $573 \mathrm{~K}$, with a possible increase at higher temperatures. 

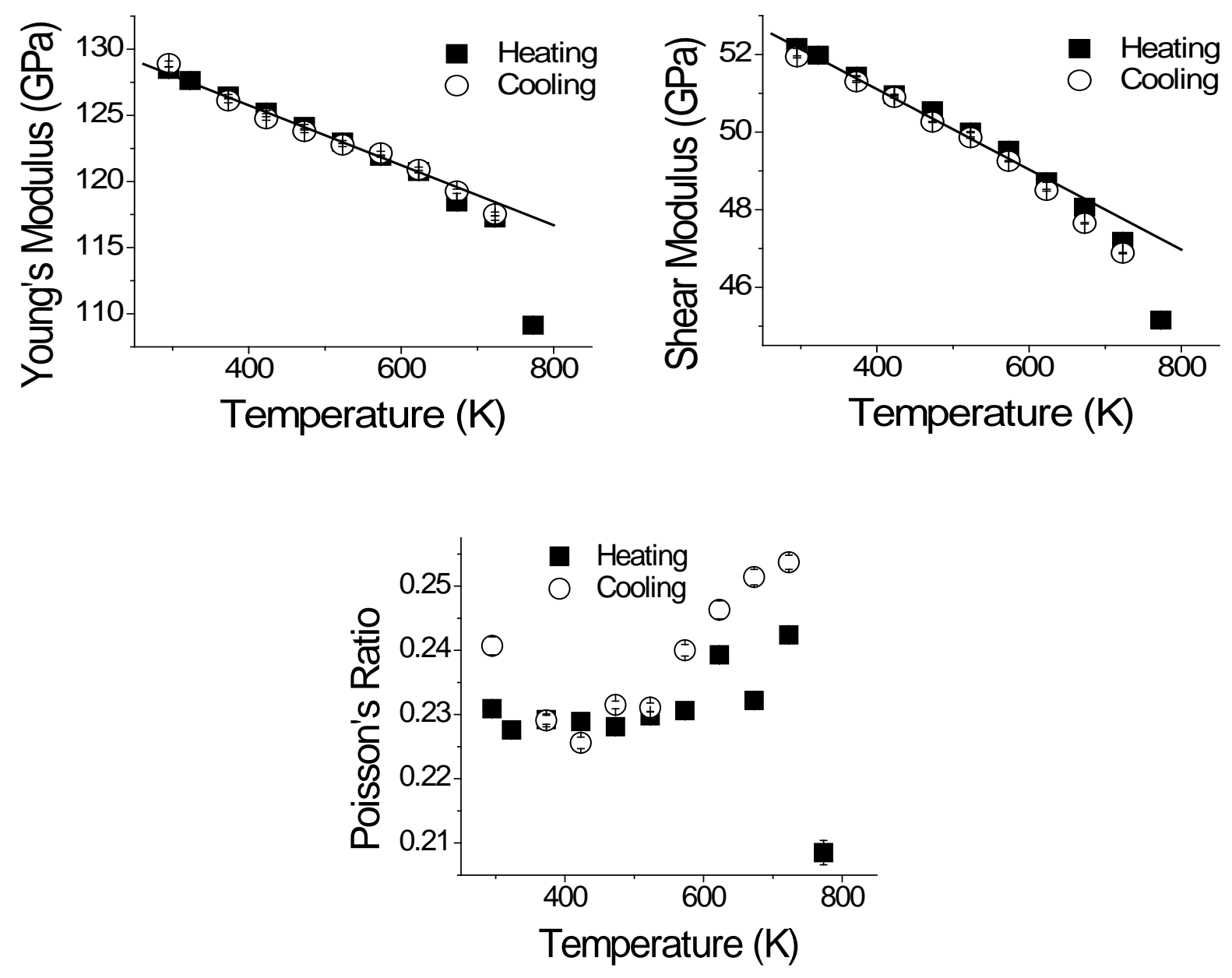

Figure 10.13. SKD-Wet4 - The Young's modulus and shear modulus of SKD-Wet4, $\mathrm{a}$ and $\mathrm{b}$, changed linearly with temperature from room temperature until above $623 \mathrm{~K}$. The highest temperature datapoint, $773 \mathrm{~K}$, was an outlier, although the material returned to a similar measured value of all elastic moduli during cooling. Similarly, the Poisson's ratio, c, remained relatively constant around 0.230 until above $573 \mathrm{~K}$, above which it began to rise with an outlier at $773 \mathrm{~K}$, and returning to near-constant upon cooling below $573 \mathrm{~K}$.

Table 10.10. The acoustic Debye temperature, as computed from the room temperature RUS measurements of the acoustic longitudinal velocity, $\mathrm{V}_{\mathrm{L}}$, and the shear velocity, $\mathrm{V}_{\mathrm{S}}$. The mean sound velocity, $\mathrm{V}_{\mathrm{M}}$, was calculated from these measurements and used to obtained the acoustic Debye temperature, $\theta_{\mathrm{D}}$.

\begin{tabular}{|l|l|l|l|l|}
\hline & $\begin{array}{l}\text { SKD- } \\
\text { Wet1 }\end{array}$ & $\begin{array}{l}\text { SKD- } \\
\text { Wet2 }\end{array}$ & $\begin{array}{l}\text { SKD- } \\
\text { Wet3 }\end{array}$ & $\begin{array}{l}\text { SKD- } \\
\text { Wet4 }\end{array}$ \\
\hline $\mathrm{V}_{\mathrm{L}}(\mathrm{km} / \mathrm{s})$ & 4.622 & 4.602 & 4.460 & 4.551 \\
\hline $\mathrm{V}_{\mathrm{S}}(\mathrm{km} / \mathrm{s})$ & 2.750 & 2.744 & 2.595 & 2.683 \\
\hline $\mathrm{V}_{\mathrm{M}}(\mathrm{km} / \mathrm{s})$ & 3.044 & 3.037 & 2.879 & 2.973 \\
\hline$\theta_{\mathrm{D}}(\mathrm{K})$ & 318.1 & 317.4 & 303.8 & 310.6 \\
\hline
\end{tabular}




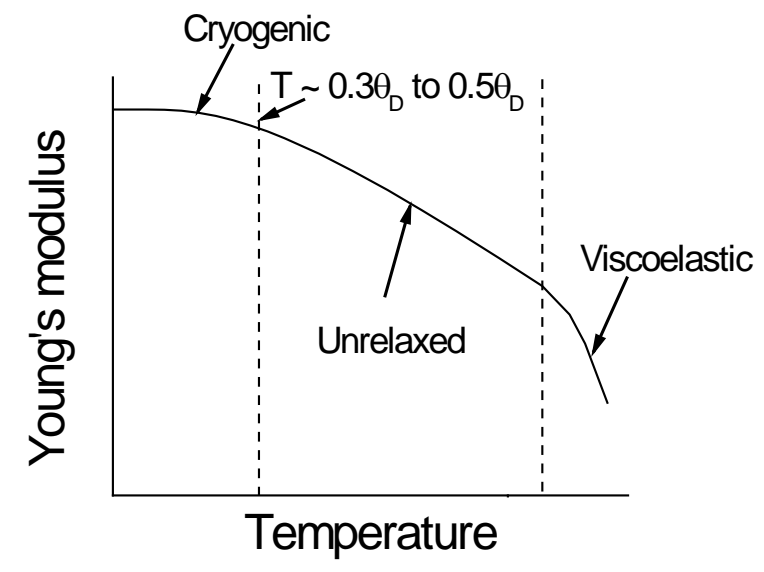

Figure 10.14. Schematic representation of temperature dependence of Young's modulus [14, 15]. 

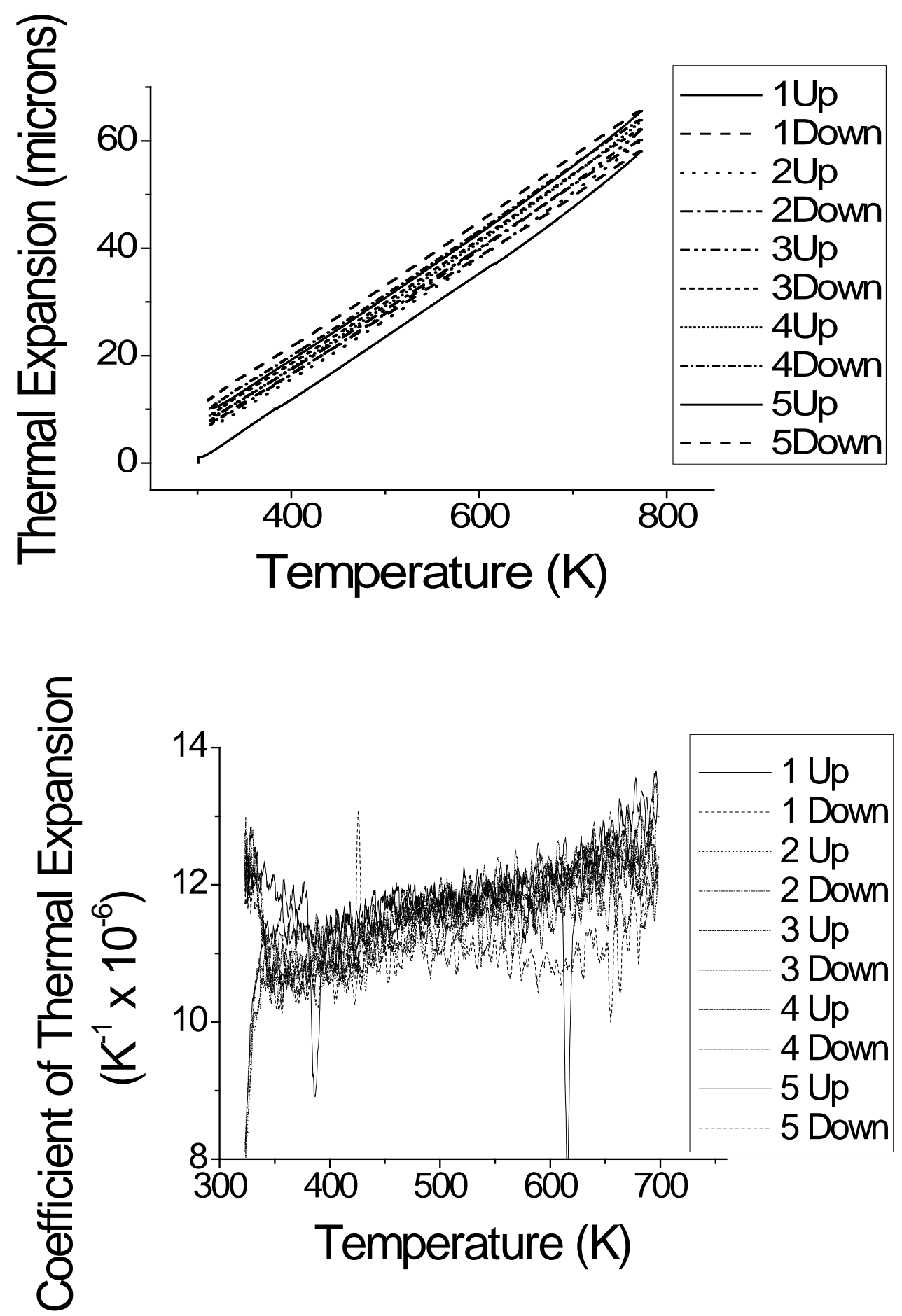

Figure 10.15. The thermal expansion measurement for specimen SKD-Wet1B, nominally $5 \times 7 \times 10 \mathrm{~mm}$. The thermal expansion rate was consistent between cycles except above $723 \mathrm{~K}$, where the specimen expanded at a faster rate on heating but not upon cooling, growing between 1 and $3 \mu \mathrm{m}$ each cycle. 


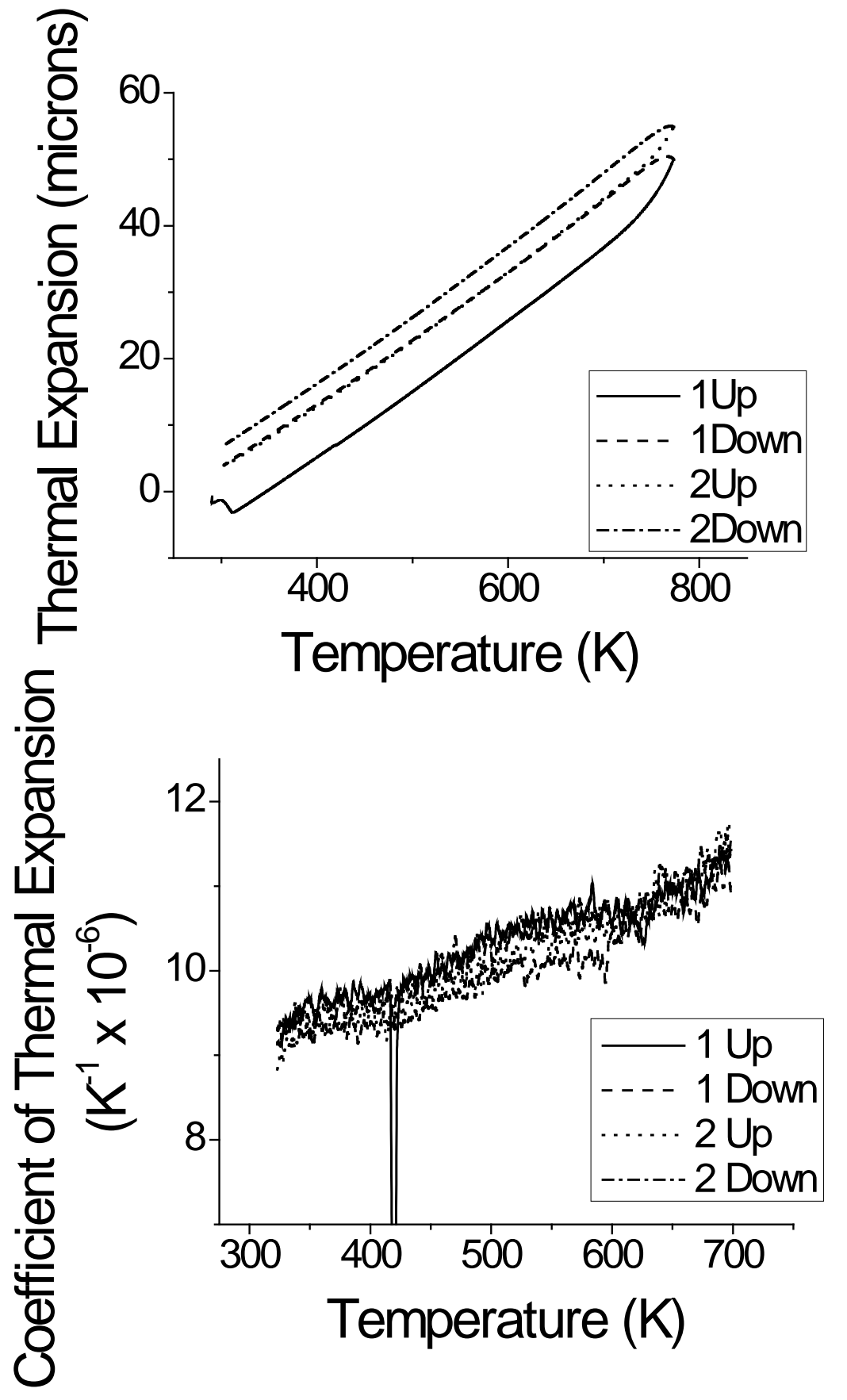

Figure 10.16. The thermal expansion measurement for specimen SKD-Wet2B, nominally $5 \times 7 \times 10 \mathrm{~mm}$. Due to the changes in SKD-Wet1B, the specimen SKD-Wet2B was cycled at a slower rate of $1.5 \mathrm{~K}$ per minute to more accurately observe any changes, resulting in fewer cycles. The thermal expansion rate was consistent between cycles except above $723 \mathrm{~K}$, where the specimen expanded at a faster rate on heating but not upon cooling, growing between 3 and $8 \mu \mathrm{m}$ each cycle. 

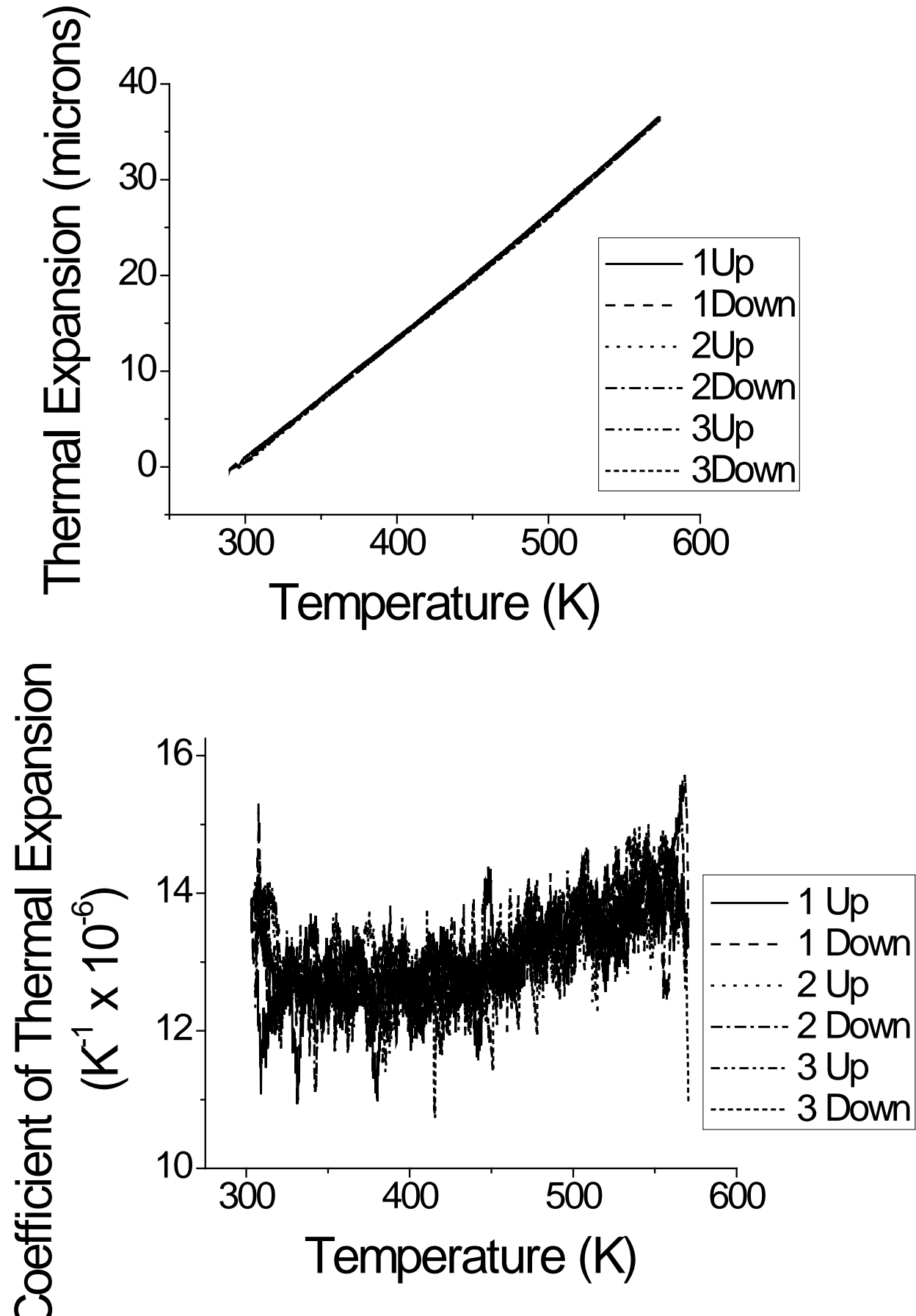

Figure 10.17. The thermal expansion measurement for specimen SKD-Wet3J, composition $\mathrm{Ce}_{0.9} \mathrm{Fe}_{3.5} \mathrm{Co}_{0.5} \mathrm{Sb}_{12}$, nominally $5 \times 7 \times 10 \mathrm{~mm}$. The thermal expansion rate was measured to $573 \mathrm{~K}$ in an effort to minimize any new phase development as was observed by X-ray above $573 \mathrm{~K}$. The resulting cycling was the most consistent of all runs between cycles, shrinking by less than $0.4 \mu \mathrm{m}$ over the entire test. 


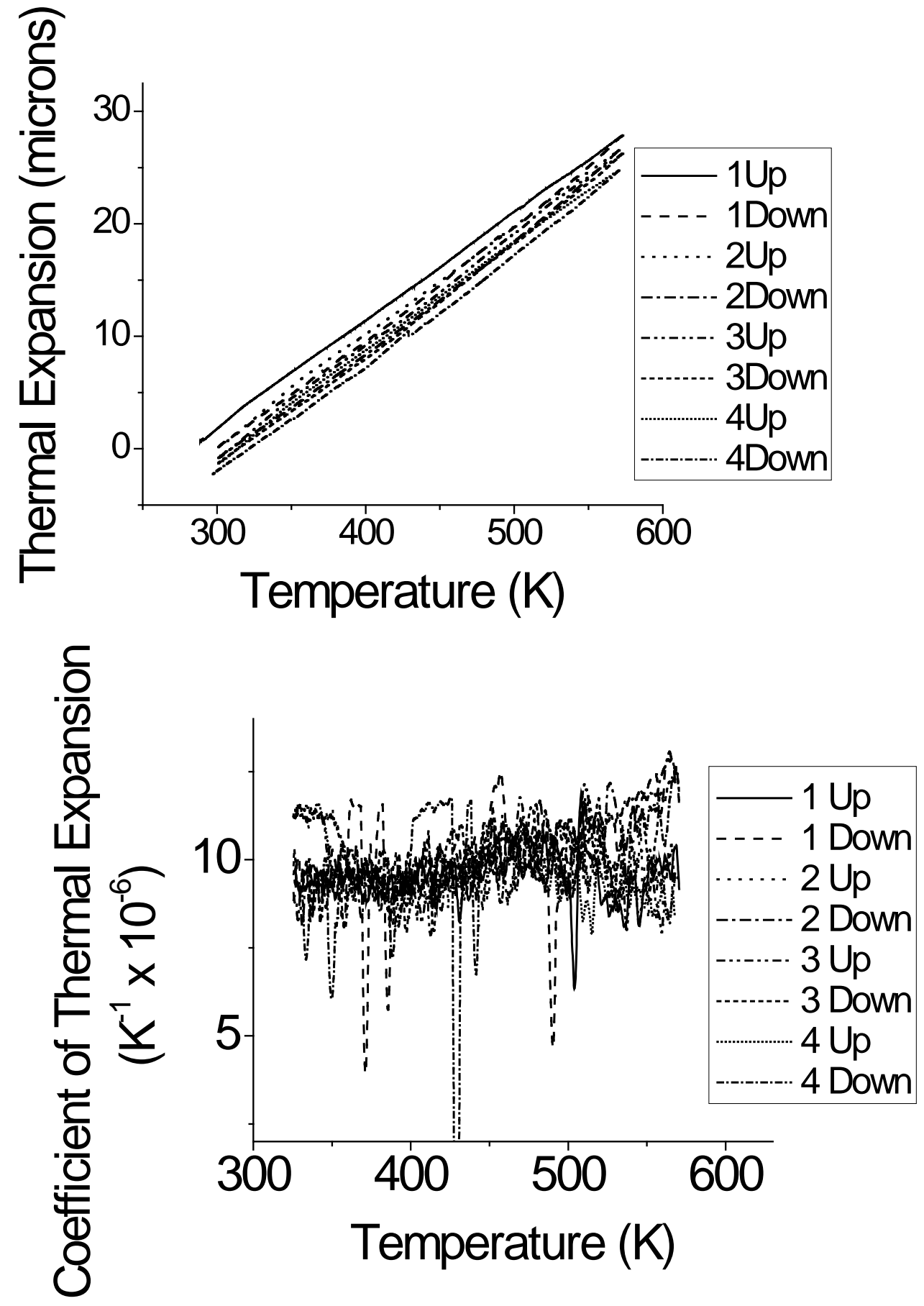

Figure 10.18. The thermal expansion measurement for specimen SKD-Wet4B, nominally 5 x 7 $\mathrm{x} 10 \mathrm{~mm}$. The thermal expansion rate was measured to $573 \mathrm{~K}$ in an effort to minimize large thermal expansions during heating above $573 \mathrm{~K}$. Several jogs were noted, particularly during the cooling segment of tests where the measured length suddenly grew. 


\section{Phase III}

\section{Introduction}

In the previous Phase II effort we focused on a combination of materials, research and experiments that modeled real-life conditions to test the performance of the thermoelectric couples. Improvements were made to the couple, module and generator designs. Two significant roadblocks to feasibly manufacturing and implementing TEGs were identified. First, the risk of a broken couple was found to pose a significant threat to the overall power production of the entire generator. The couples and modules need to be wired in series to maximize voltage generation. If a single broken couple disrupts the electrical circuit, it negates the power generation of the other couples. Secondly, the couple degradation and failures that occurred during testing compromised generator longevity. To address these issues, the focus of Phase III was two-fold: reduce the number of couple breakages that occur during testing and to mitigate the effect of a broken couple on the overall power generation.

\subsection{Finite Element Analysis}

The module failures that occurred during the airtorch tests (reported in Phase II) raised serious concerns about the longevity of the generator. To investigate the material interactions that occur during thermal cycling, a Finite Element Analysis was performed using Abaqus, in two stages. First, a thermal analysis was performed. Convective heat transfer was assumed at the hot-shoe fin surfaces and in the water passage of the copper base-plate. All the other surfaces were assumed to be insulated. The finite element meshed geometry for the TE module is shown in Figure 1.1. The mesh is forced to be denser in the areas of interest where the different material interfaces exist.

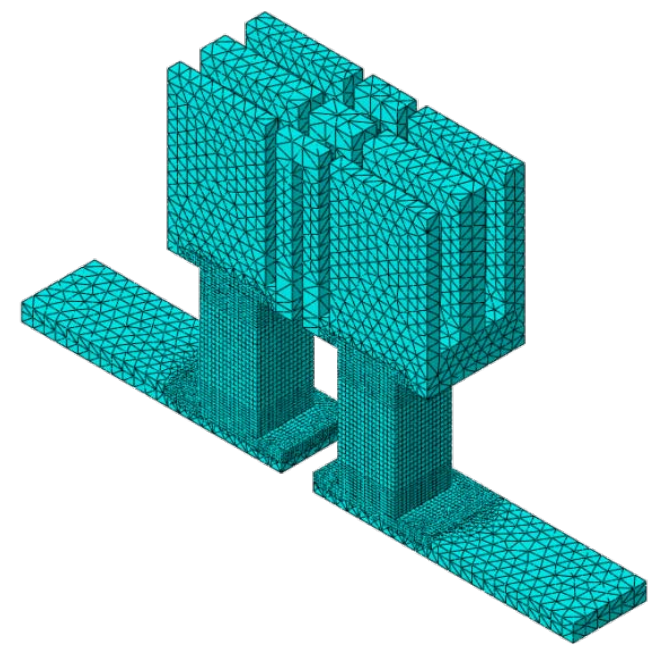

Figure 1.1. Mesh for TE module.

Secondly, a structural analysis was performed in four steps (Table 1.1) to simulate the cyclic thermal loading of the TE module experiences during operation. The analyses considered nonlinearities and accounted for plasticity. It was assumed that the TE legs are stress-free at the beginning of the analysis, i.e. there were no residual stresses from the hot-pressing process and 
cutting of the legs. The obtained temperature distribution is shown in Figure 1.2. Some analysis times for a model with a quarter of a million nodes are reported in Table 1.2.

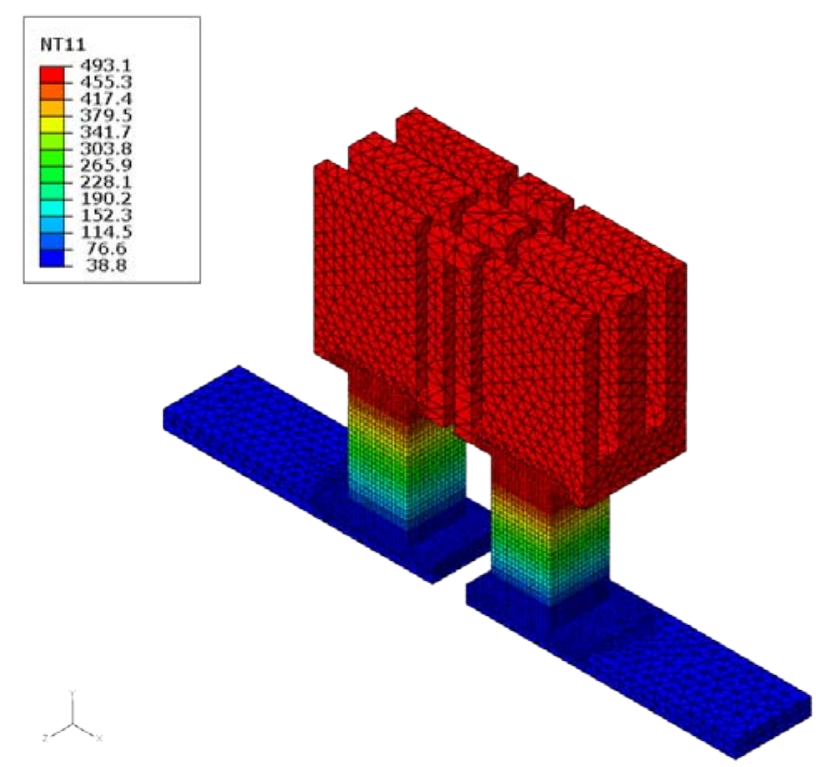

Figure 1.2. Operating temperature distribution of TE module (deg. C).

Table 1.1. Steps for structural analysis.

\begin{tabular}{|c|c|}
\hline Step & Temperature Field \\
\hline Cycle 1 & Operating Temperature from thermal analysis \\
\hline Cycle 2 & Cooled-down at 30 deg. C \\
\hline Cycle 3 & Operating Temperature from thermal analysis \\
\hline Cycle 4 & Cooled-down at 30 deg. C \\
\hline
\end{tabular}

Table 1.2. Simulation Times

\begin{tabular}{|c|c|c|}
\hline Analysis & CPU Time (s) & Wall Clock Time (s) \\
\hline Thermal & 800 & 896 \\
\hline Structural & 97377 & 119175 \\
\hline
\end{tabular}

\subsection{Results}

The simulation results are presented in this section. Keeping in mind the assumptions made in building the model, these results should be treated as descriptive rather than prescriptive. The overall leg behavior might not have quantitative agreement with the actual leg behavior, but it does have a qualitative agreement, as shown when comparing with actual test legs.

Figure 1.3 through Figure 1.8 show the von Mises stresses at the legs over the four cycles simulated. Figure 1.9 and Figure 1.10 show the second principal stresses at Cycle 2 and Cycle 4, when the TE module is cooled to $30^{\circ} \mathrm{C}$. In these figures, the stresses are in $\mathrm{MPa}$. The magnification factor for the deformation is 175 . The front leg is the P-type with the N-type is at the back. Figure 1.11 and Figure 1.12 show an actual P-type leg, unloaded and loaded respectively. Figure 1.13 shows a loaded N-type leg. 
Focusing on the P-type leg, where failure occurs at the skutterudite-material B interface, material $\mathrm{B}$ has the highest yield strength, whereas skutterudite has the lowest. At the same time, the thermal expansion coefficient of material B is higher by 1.5 units per Kelvin. Material B also has a higher thermal expansion coefficient than material A by 3.6 units per Kelvin.

During the heating cycle all of the materials expand. The expansion amount decreases moving down along the leg (temperatures decrease). Hence, the material $\mathrm{B}$ tends to expand more during the heating cycle, since it is hotter and also has a higher thermal expansion coefficient. Material $\mathrm{B}$ pulls the neighboring materials along the interface. At the material A interface larger stresses are observed since it is a strong material. At the skutterudite interface lower stresses are observed as the pulling action of the material $\mathrm{B}$ yields the material.

On the cool down cycle, the material B, which suffers little plasticity, wants to contract back to its original shape. The skutterudite has suffered unrecoverable expansion and wants to stay deformed. This creates a bulge (due to skutterudite plasticity) at the material B-skutterudite interface. This bulge is observed in experiments (Figure 1.12). Hence, these opposing desires of the two materials result in high stresses. Specifically, looking at the principal stresses, the second principal stress is the highest at the material B-skutterudite interface (Figure 1.9). These high stresses can result in fatigue after multi-cycle loading and lead to separation at the interface. The stress distributions after subsequent thermal loading cycles show little difference, however, the magnitude range increases (Figure 1.9 and Figure 1.10). Furthermore, another reason for failure can be due to crack initiation and growth. Imperfections in the surface finish, introduced during the cutting process of the legs, combined with the high stress concentrations, can promote crack growth and separation. A similar behavior is seen with the $\mathrm{N}$-type leg at the material A-metal foil-skutterudite interface. In this case, the metal foil has the lowest thermal expansion coefficient at 5.8 units per Kelvin and it is a strong and elastic material. Metal foil opposes the expansion of both the material A above it and the skutterudite below it. In this case a notch is created and is observed in experiments (Figure 1.13)

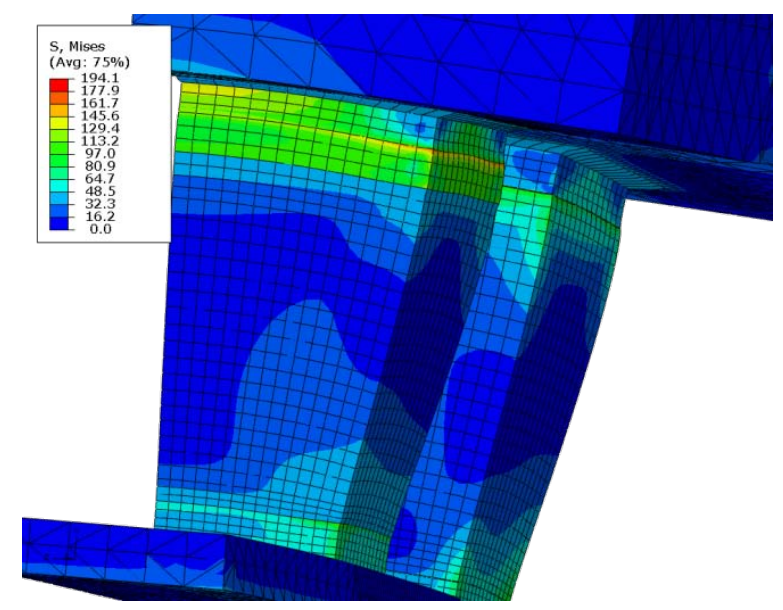

Figure 1.3. Cycle 1 - stresses at $50 \%$ of the operating temperature.

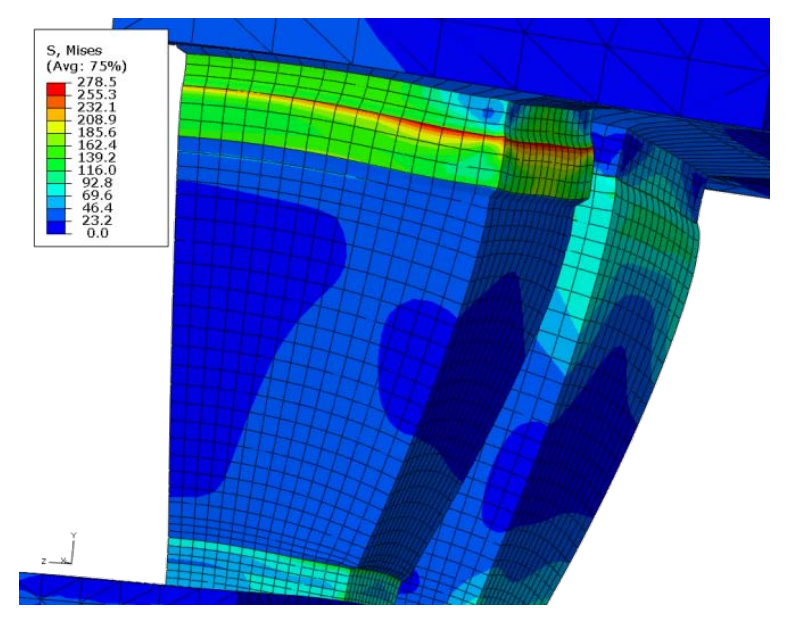

Figure 1.4. Cycle 1 - stresses at operating temperature. 


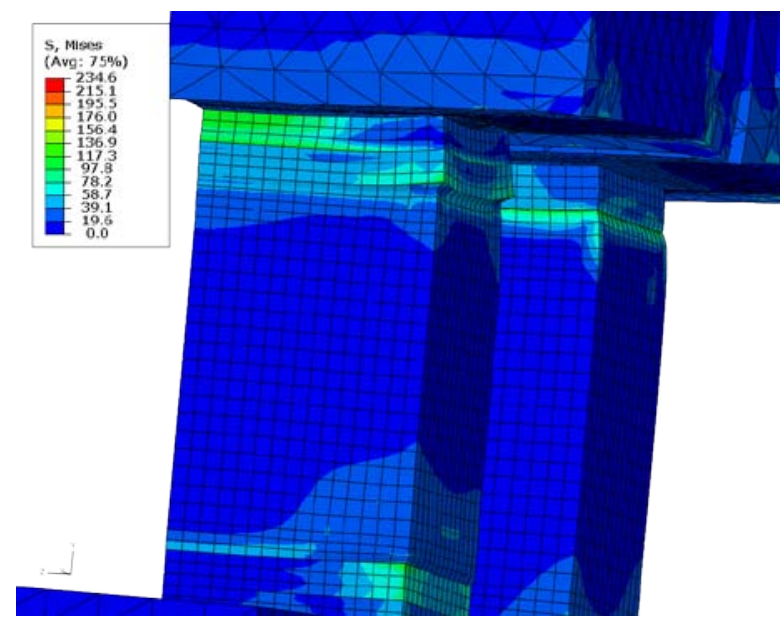

Figure 1.5. Cycle 2 - stresses at halfway to cooling to $30 \mathrm{deg}$. C.

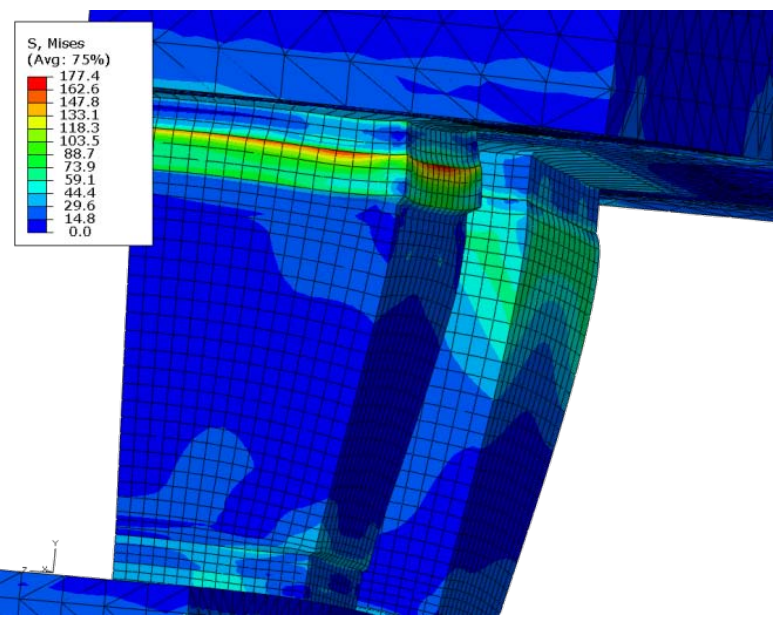

Figure 1.6. Cycle 2 - stresses after cooling. to 30 deg. C. Evidence of plasticity is seen in both legs.

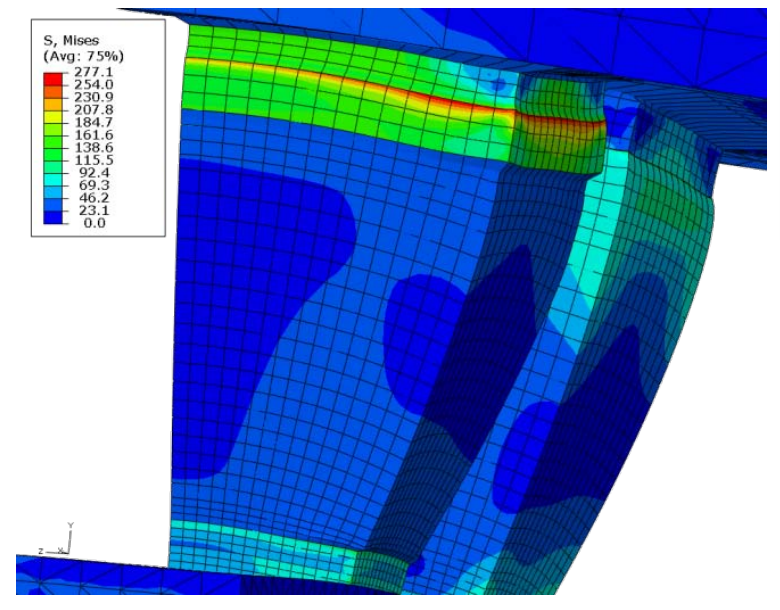

Figure 1.7. Cycle 3 - stresses at operating temperature. to $30 \mathrm{deg}$. C. Evidence of plasticity is seen in both legs.

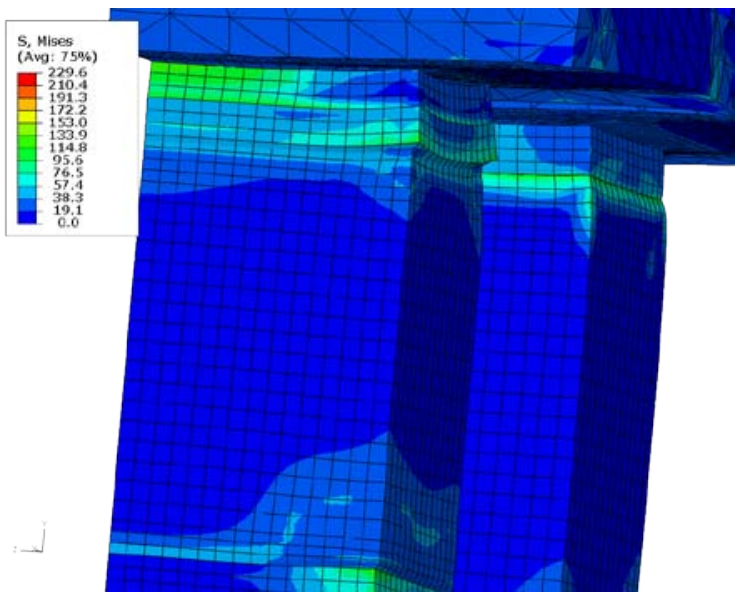

Figure 1.8. Cycle 4 - stresses after Cooling to $30 \mathrm{deg}$. C. to $30 \mathrm{deg}$. C. Evidence of plasticity is seen in both legs. 


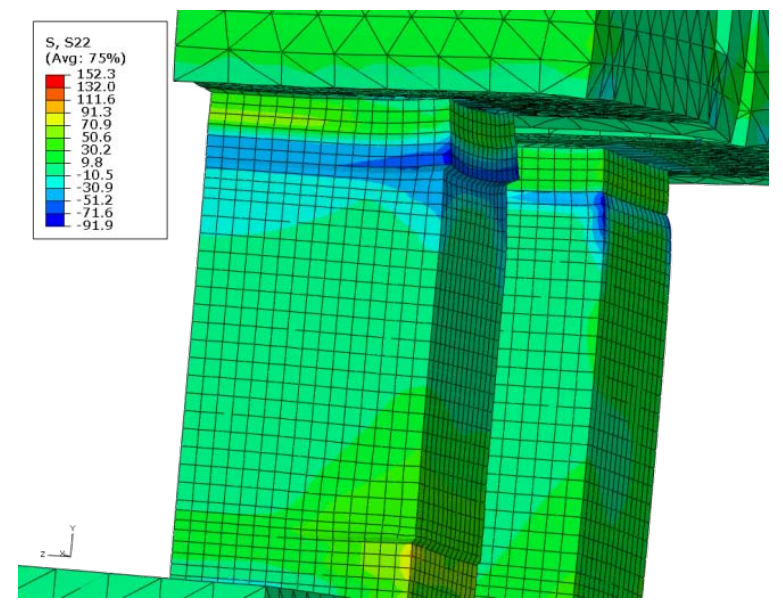

. Figure 1.9. Cycle 2 - second principal stresses after cooling down to 30 deg. $\mathrm{C}$ to $30 \mathrm{deg}$. C. Evidence of plasticity is seen in both legs.

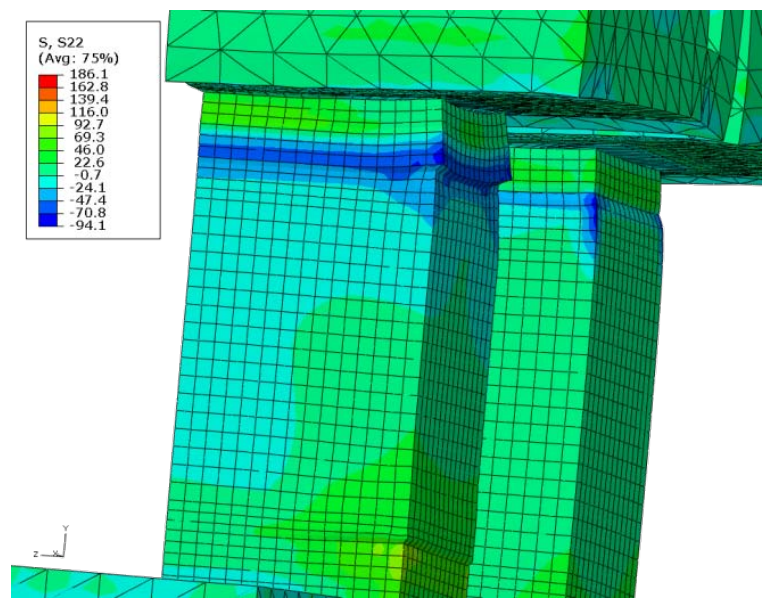

Figure 1.10. Cycle 4 - second principal stresses after cooling down to 30 deg. C.

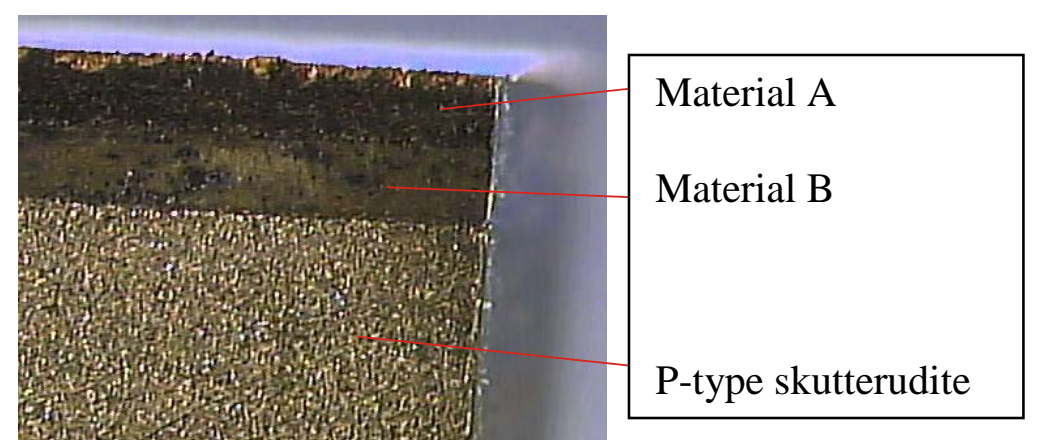

Figure 1.11. P-type leg seen under a microscope before it undergoes any loading. 


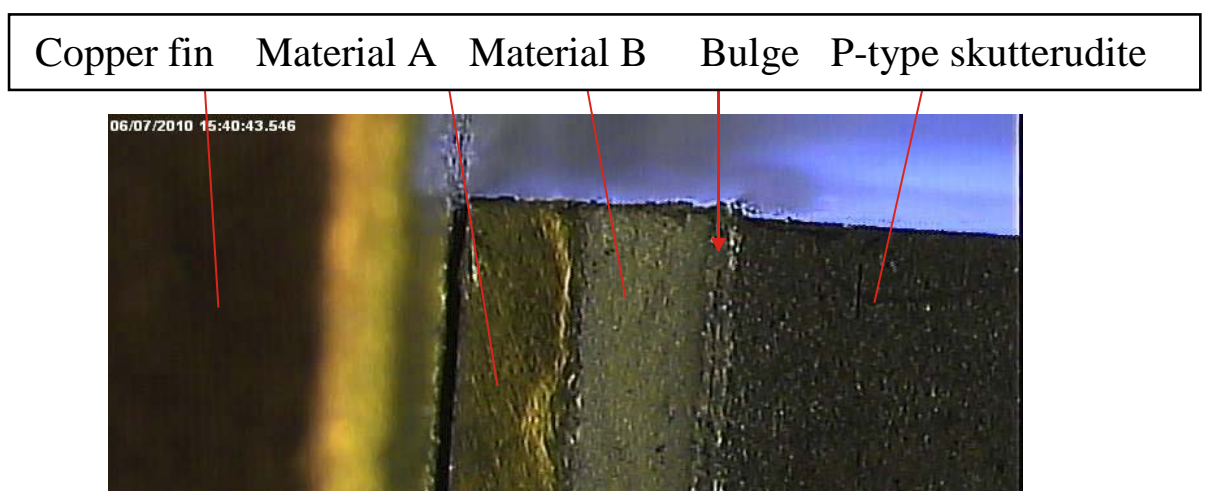

Figure 1.12. P-type leg seen under a microscope after four thermal loading cycles.

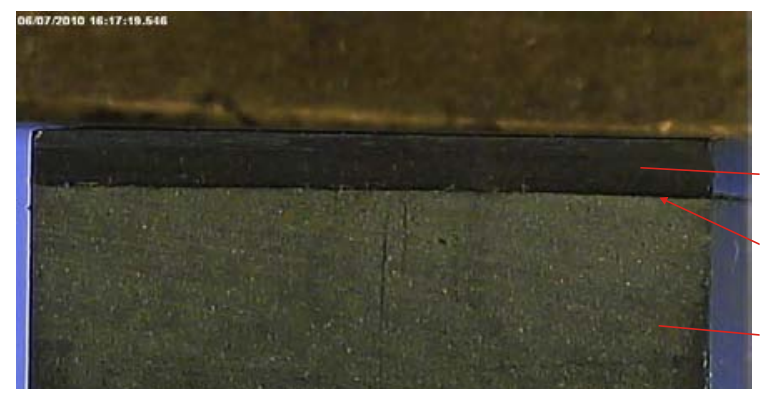

Copper fin
Material A
Material B
N-type skutterudite

Figure 1.13. N-type leg seen under a microscope after four thermal loading cycles.

To address the high level of thermal stress at the skutterudite and material B interface, the leg size was reduced from $3.5 \times 7 \times 7 \mathrm{~mm}$ to $3.5 \times 3.5 \times 7 \mathrm{~mm}$. Using this new leg size, $5 \mathrm{~W}$ modules were fabricated from 10 couples that produce roughly $0.5 \mathrm{~W}$ each. This reduction in leg size also had the benefit of doubling the voltage produced for the same power output. Integrating this new leg size into the current fabrication methods required a few modifications of the current process. These modifications will be described in the next chapter. Table $\mathbf{1 . 3}$ compares the performance of the $5 \mathrm{~W}$ module made from the $10,0.5 \mathrm{~W}$ couples of the new leg size, versus the performance of a $5 \mathrm{~W}$ module made from $5,1 \mathrm{~W}$ couples of the old leg size.

Table 1.3. Performance comparison of leg sizes.

\begin{tabular}{|c|c|c|c|c|c|}
\hline & $\begin{array}{c}\text { Average } \\
\text { Resistance } \\
\text { (Ohm) }\end{array}$ & $\begin{array}{c}\text { Average Peak } \\
\text { Power } \\
\text { (W) }\end{array}$ & $\begin{array}{c}\text { Max } \\
\text { Power } \\
\text { (W) }\end{array}$ & $\begin{array}{c}\text { Average Open } \\
\text { Circ. Voltage } \\
\text { (V) }\end{array}$ & $\begin{array}{c}\text { Max Open } \\
\text { Circ. Voltage } \\
\text { (V) }\end{array}$ \\
\hline $\begin{array}{c}\text { 3.5mmX7mm leg } \\
\text { (old) }\end{array}$ & 0.034 & 5.233 & 6.18 & 0.725 & 0.763 \\
\hline $\begin{array}{c}\text { 3.5mmX3.5mm leg } \\
\text { (new) }\end{array}$ & 0.101 & 5.312 & 5.778 & 1.47 & 1.575 \\
\hline
\end{tabular}




\section{Skutterudite Materials}

\subsection{Material Synthesis}

Materials for both the $\mathrm{N}$ and P-type skutterudite were weighed inside the single glove box and transferred to a graphite crucible. The total batch size is approximately $100 \mathrm{~g}$ for both the $\mathrm{N}$ and P-type material. The graphite crucible was then placed inside a $50 \mathrm{~mm}$ quartz tube and put onto the sealing line. The quartz tubes were left on the sealing line until the pressure reached $3 \times 10^{-6}$ torr. The quartz tubes were then flame sealed and placed inside a tube furnace. The furnace was then programmed to heat to $1100^{\circ} \mathrm{C}$ at a rate of $10^{\circ} \mathrm{C} / \mathrm{min}$. The material was then held at $1100^{\circ} \mathrm{C}$ for approximately 12 hours and then air quenched. After air quenching, the material is annealed for 48 hrs at $700^{\circ} \mathrm{C}$ for the N-type and $740^{\circ} \mathrm{C}$ for the P-type.

\subsection{Powder Processing}

After annealing, the material is transferred into the powder processing glove box and removed from the quartz tube and graphite crucible. The ingot is then crushed and ground using the motorized mortar and pestle. After grinding for 5 minutes, the powder is sieved for 30 minutes using a $75 \mu \mathrm{m}$ test sieve. Material that does not pass through the sieve is reground for 5 minutes and sieved again. This process is repeated until approximately $99 \%$ of the material has passed through the $75 \mu \mathrm{m}$ test sieve.

All the material is then put into a $500 \mathrm{ml}$ stainless steel ball mill jar along with $7-20 \mathrm{~mm}$ stainless steel balls. The material is then ball milled for 3 hours at $110 \mathrm{rpm}$. Next, the powder is removed from the jar and placed inside a glass jar until further use.

\subsection{Die Preparation}

The loading of the powder material is done inside of the powder processing glove box. The die is first prepared outside the glove box by lining the inside of the die with grafoil. The contacting surfaces of the die bottom and plunger are coated with boron nitride spray and allowed to dry for 15 minutes. Two pieces of $0.05 \mathrm{~mm}$ copper foil and two pieces of $0.0016 \mathrm{~mm}$ metal foil (the same diameter as the plunger) are cut and cleaned with acetone. One of the pieces of copper is glued to the die bottom and then inserted into the die. The die, along with the foil pieces are loaded into the glove box where the powder materials are loaded.

Table 2.1 and Table 2.2 show the loading sequence for each hot pressed material. The powder of each material is poured into the die and then cold pressed using a hydraulic press located inside of the glove box. This process is repeated until all powders and foil have been loaded into the die.

Table 2.1. Powder and foil layers for N-type hot pressed billet.

\begin{tabular}{|c|c|}
\hline Material & Thickness, mm \\
\hline Copper foil & 0.05 \\
\hline Material A powder & 0.60 \\
\hline Metal foil & 0.016 \\
\hline N-SKD powder & 6.1 \\
\hline Metal foil & 0.016 \\
\hline Material A powder & 0.60 \\
\hline Copper foil & 0.05 \\
\hline
\end{tabular}


Table 2.2. Powder and foil layers for P-type hot pressed billet.

\begin{tabular}{|c|c|}
\hline Material & Thickness, $\mathbf{~ m m}$ \\
\hline Copper foil & 0.05 \\
\hline Material A powder & 0.30 \\
\hline Material B powder & 0.30 \\
\hline Metal foil & 0.016 \\
\hline N-SKD powder & 5.9 \\
\hline Metal foil & 0.016 \\
\hline Material B powder & 0.30 \\
\hline Material A powder & 0.60 \\
\hline Copper foil & 0.05 \\
\hline
\end{tabular}

\subsection{Hot Pressing}

All dies are loaded into the hot press immediately after they are removed from the glove box. The hot press is then allowed to pump down for approximately 1 hour or until it reaches 100 millitorr. It is then back filled with high purity argon (99.999\%) and allowed to pump down overnight ( 12 hours). The hot pressing and metallization of the $\mathrm{N}$ and P-type material has been standardized. During the hot pressing the temperature and pressure have been optimized in order to produce strong and robust legs. Metalizing both sides in copper during the hot pressing procedure has improved the hot side bonding process. Over 70 pucks of both the $\mathrm{N}$ and P-type have been hot pressed to date. Our largest $50 \mathrm{~mm}$ die produces 112 legs that are diced into $3.5 \times 3.5 \times 7 \mathrm{~mm}$ legs (Figure 2.1). This is twice as many legs as the previous leg size, which was $3.5 \times 7.7 \mathrm{~mm}$. The temperature and press profiles for both the $\mathrm{N}$ and P-type material are shown in Tables 2.3 and 2.4.

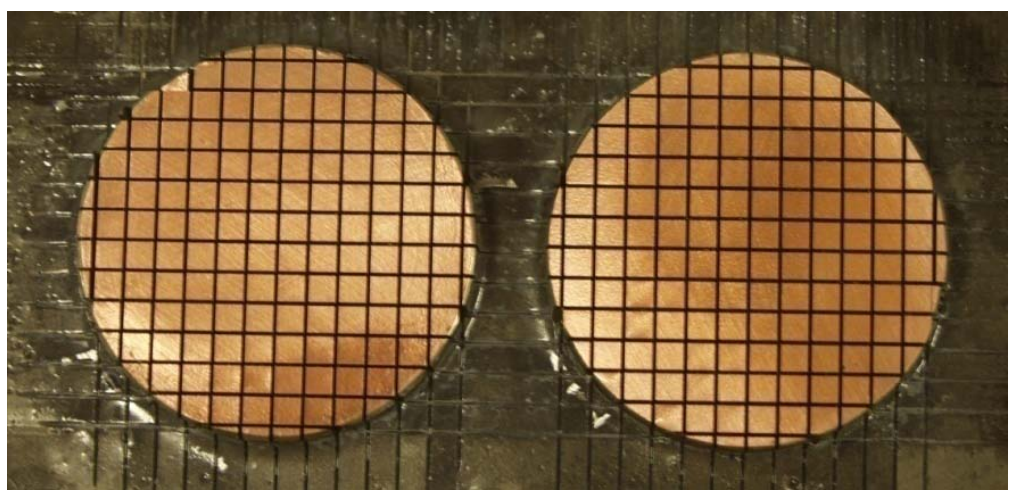

Figure 2.1. Hot pressed $\mathrm{N}$ and P-type metalized skutterudite pucks diced into $3.5 \times 3.5 \times 7 \mathrm{~mm}$ legs. 
Table 2.3. Temperature and force profile for the N-type SKD hot pressing.

\begin{tabular}{|c|c|c|c|c|c|c|}
\hline Time - min & 20 & 20 & 10 & 120 & 20 & 120 \\
\hline Temp - ${ }^{\mathbf{C}}$ & 250 & 500 & 730 & 730 & 600 & 50 \\
\hline Force - kg & 0 & 15500 & 15500 & 15500 & 0 & 0 \\
\hline
\end{tabular}

Table 2.4. Temperature and force profile for the P-type SKD hot pressing.

\begin{tabular}{|c|c|c|c|c|c|c|}
\hline Time - min & 20 & 20 & 10 & 120 & 20 & 120 \\
\hline Temp - ${ }^{\mathbf{C}} \mathbf{C}$ & 250 & 500 & 660 & 660 & 550 & 50 \\
\hline Force - kg & 0 & 15500 & 15500 & 15500 & 0 & 0 \\
\hline
\end{tabular}

\subsection{Pre-Anneal Testing}

Tests were conducted on several of the $\mathrm{N}$ and P-type skutterudite legs to determine the effect of annealing on the electrical properties of the material. As shown in Figures 2.2 and 2.3, prior to annealing, $3 \mathrm{~N}$-type legs and $3 \mathrm{P}$-type legs were electronically scanned.
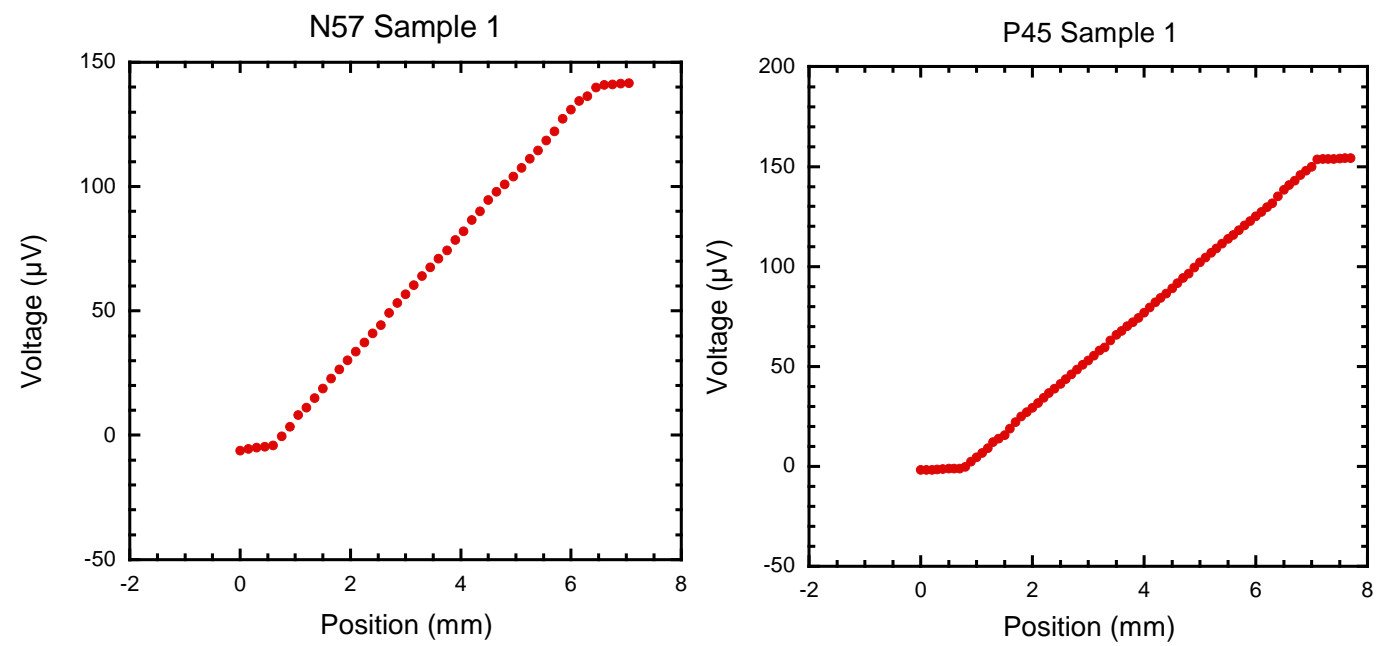

Figure 2.2. Electronic scans of $\mathrm{N}$ and P-type skutterudite legs before annealing, Test 1. 

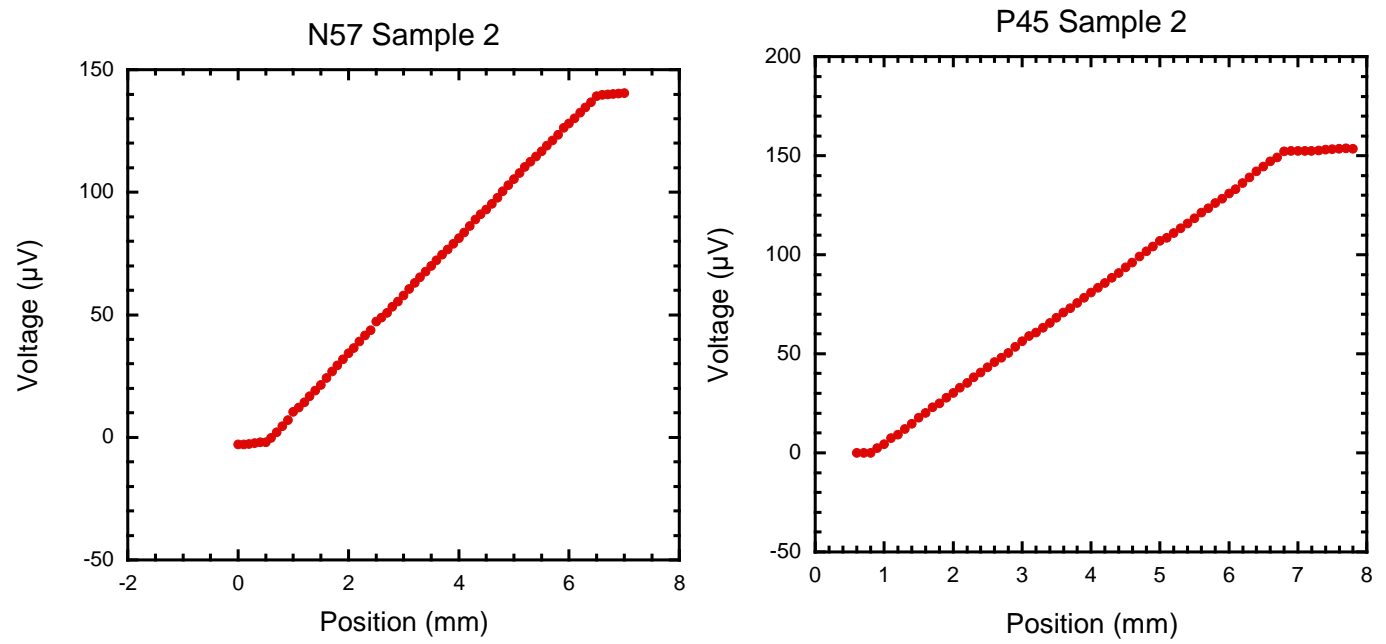

Figure 2.3. Electronic scans of $\mathrm{N}$ and P-type skutterudite legs before annealing, Test 2.
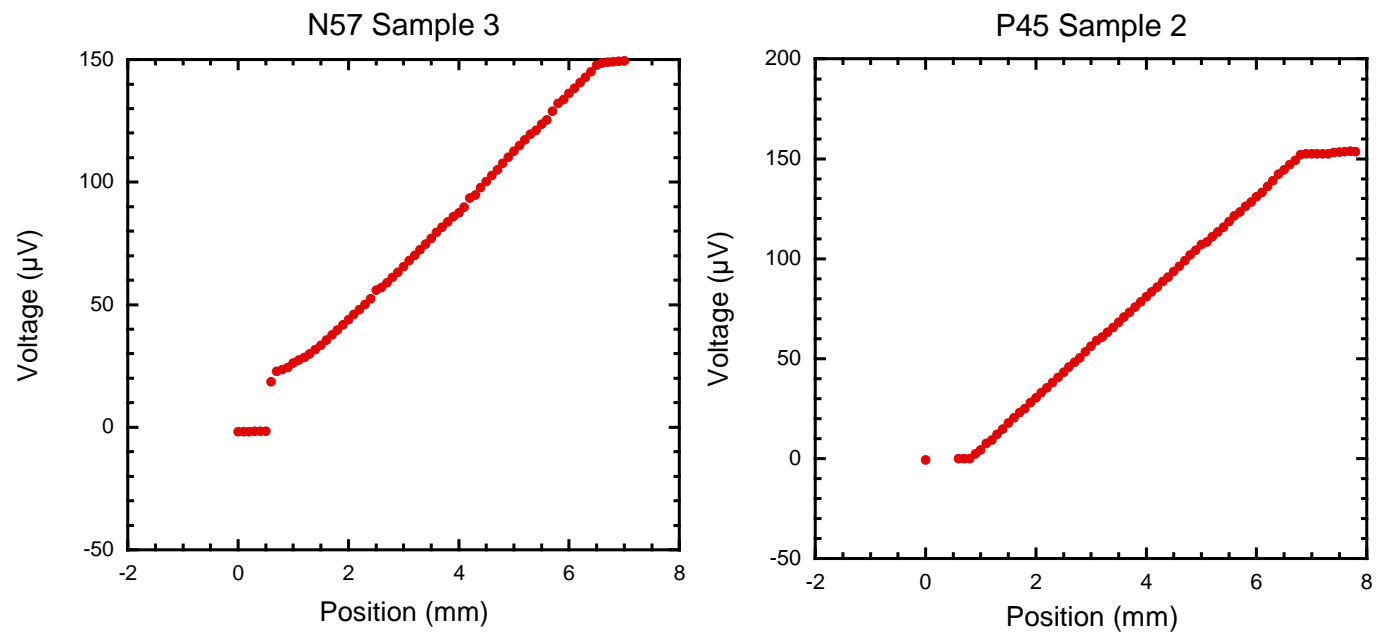

Figure 2.4. Electronic scans of $\mathrm{N}$ and P-type skutterudite legs before annealing, Test 3.

\subsection{Annealing}

Before annealing the legs, they were heated up on a hot plate and solder wick was used to remove the solder from the copper ends of the leg. A pair of legs (1 N and 1 P-type was then flame sealed in a $13 \mathrm{~mm}$ OD quartz tube after the pressure inside the tube reached below $1 \times 10^{-5}$ torr. In the first anneal test, the furnace was set to ramp up to $700^{\circ} \mathrm{C}$ in 30 minutes and then shut off. The results for the temperature profile are shown in Figure 2.5. It took approximately 11 hours to cool from $700^{\circ} \mathrm{C}$ to $50^{\circ} \mathrm{C}$. In the second and third anneal tests, the furnace was set to ramp to $700^{\circ} \mathrm{C}$ in 30 minutes, held at $700^{\circ} \mathrm{C}$ for 4 hours and then shut off (Figures 2.6 and 2.7). The cool down time (from $700^{\circ} \mathrm{C}$ to $50^{\circ} \mathrm{C}$ ) in both tests was approximately 12 hours. 


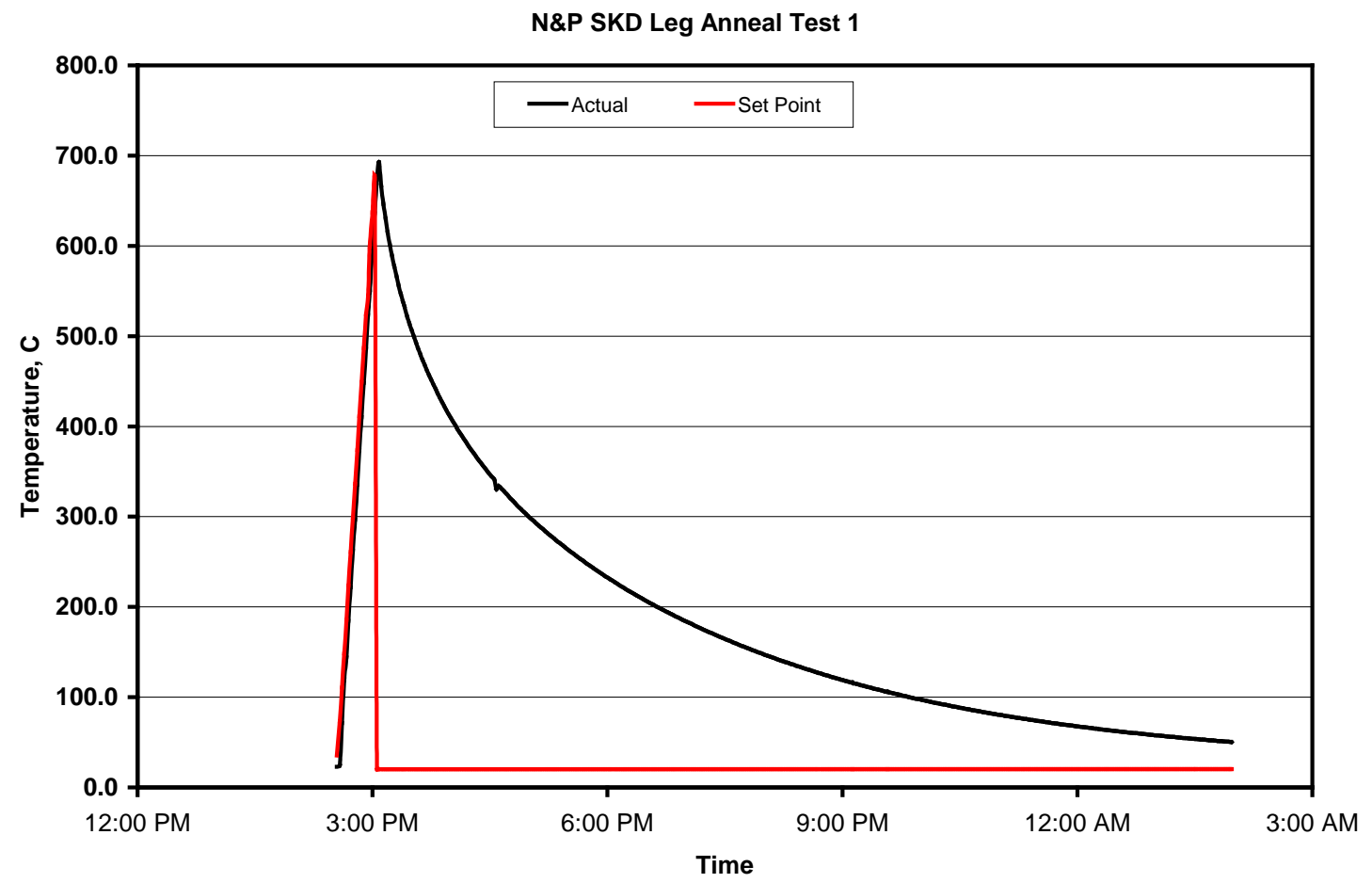

Figure 2.5. Temperature profile of the first anneal test of the $\mathrm{N}$ and P-type skutterudite legs.

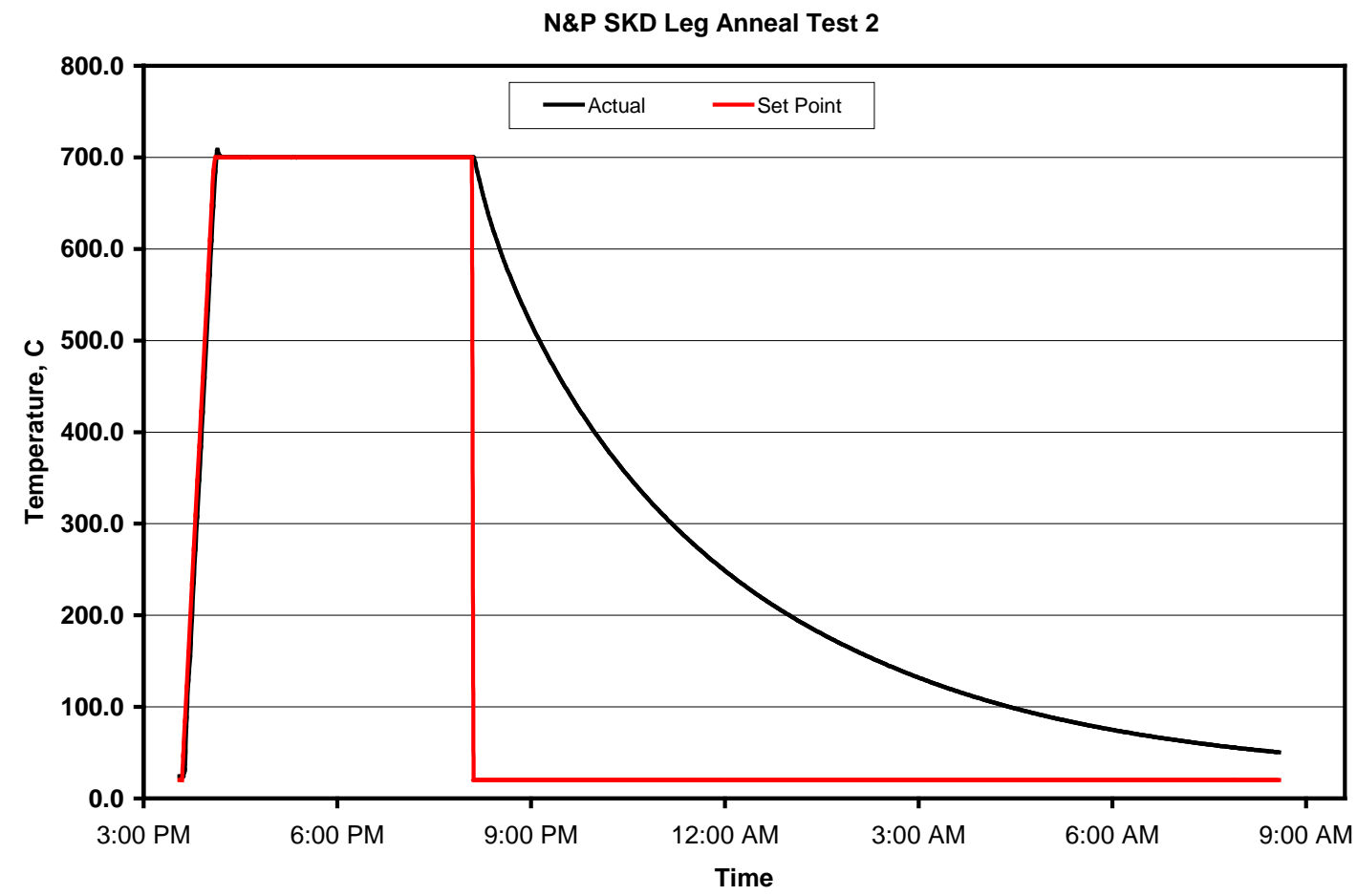

Figure 2.6. Temperature profile of the second anneal test of the $\mathrm{N}$ and P-type skutterudite legs. 


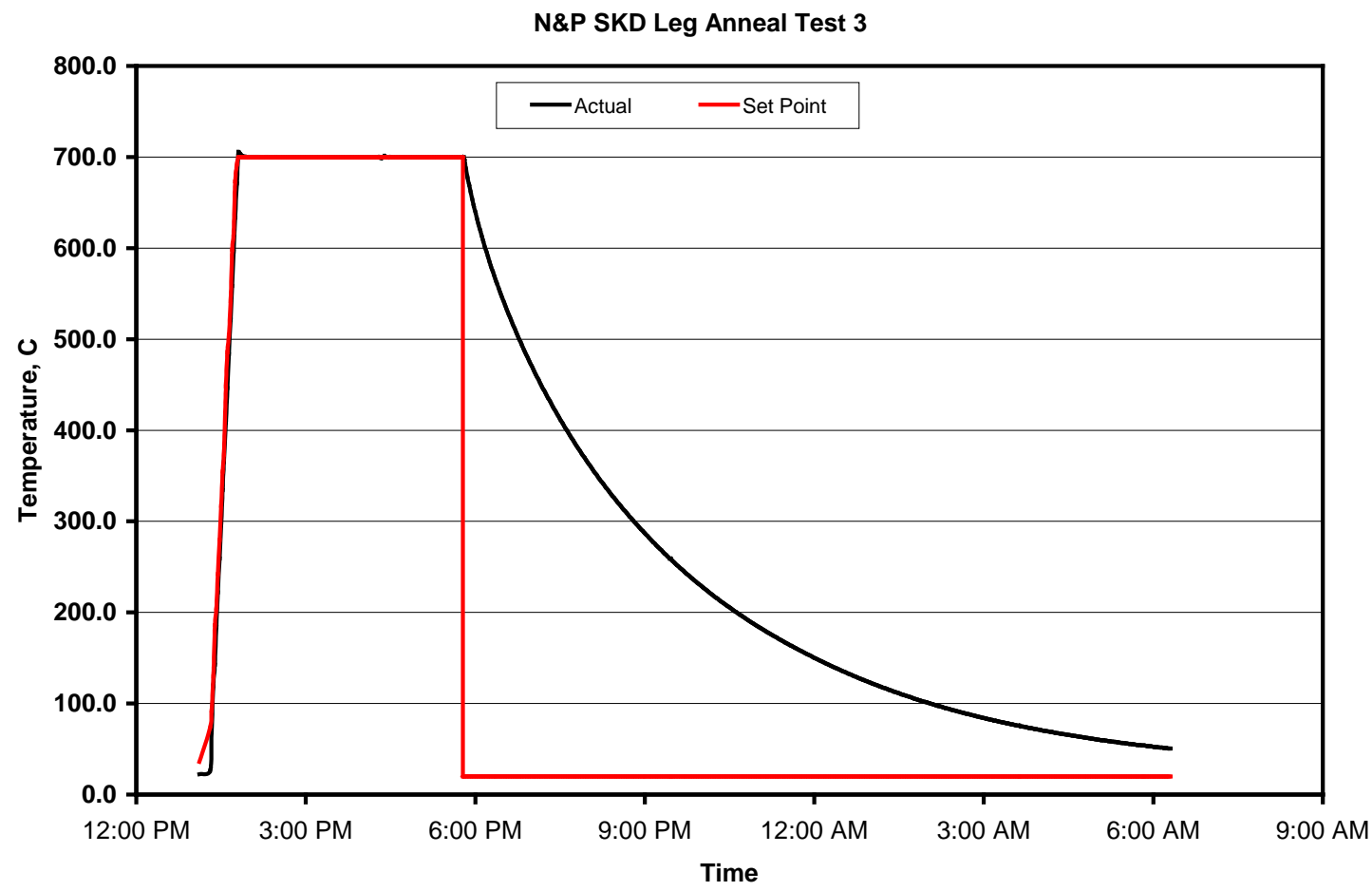

Figure 2.7. Temperature profile of the third anneal test of the $\mathrm{N}$ and P-type skutterudite legs.

\subsection{Post-Anneal Testing}

After annealing, the legs were removed from the quartz tube and again electronically scanned. The results for the 3 anneal tests are shown below in Figures 2.8 through 2.10. The results indicate that several of the legs increased in contact resistance between the metallization and the skutterudite material. In one test the metallization cracked and separated from the material, Figure 2.11.
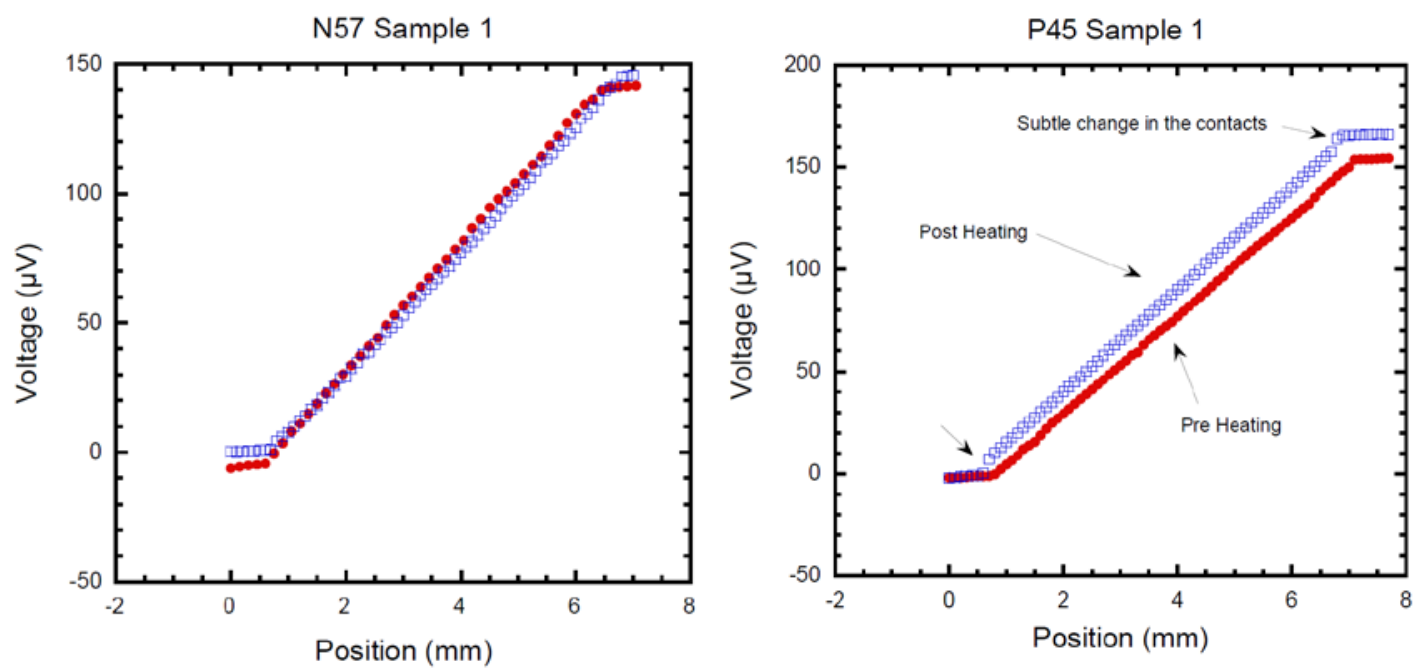

Figure 2.8. Electronic scans of $\mathrm{N}$ and P-type skutterudite legs before and after annealing, Test 1. 


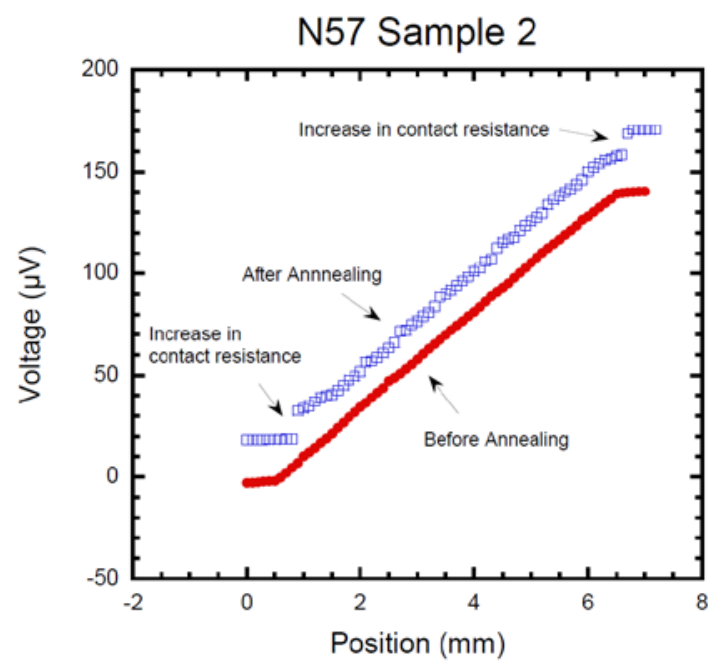

Figure 2.9. Electronic scans of N-type skutterudite leg before and after annealing, Test 2.
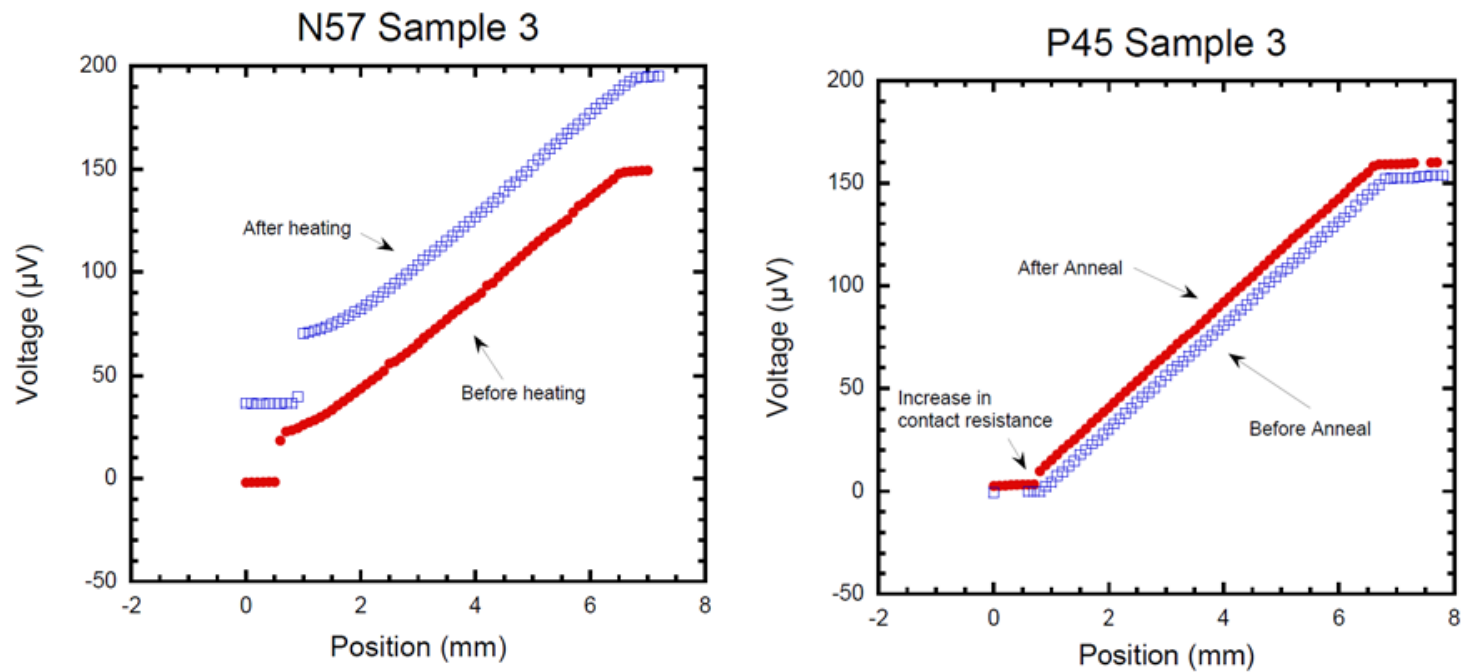

Figure 2.10. Electronic scans of $\mathrm{N}$ and P-type skutterudite legs before and after annealing, Test 3. 

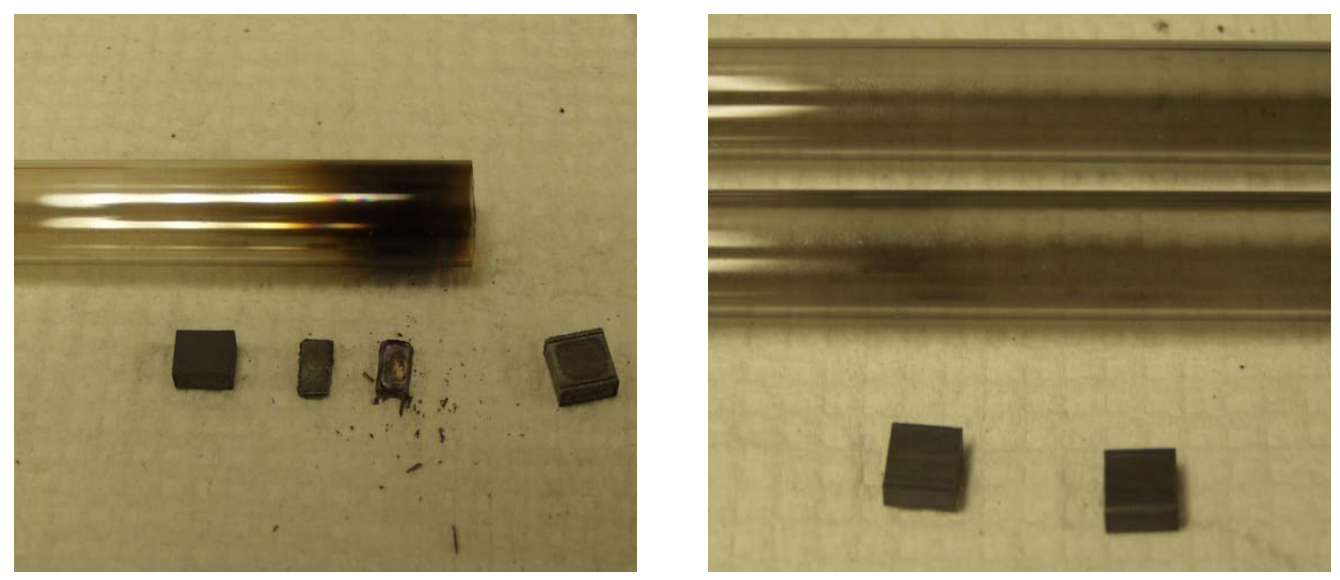

Figure 2.11. $\mathrm{N}$ and P-type skutterudite legs after annealing in Test 2 and 3.

\subsection{Transport Measurements}

Electrical and thermal transport measurements are shown in the following plots (Figures 2.122.15).
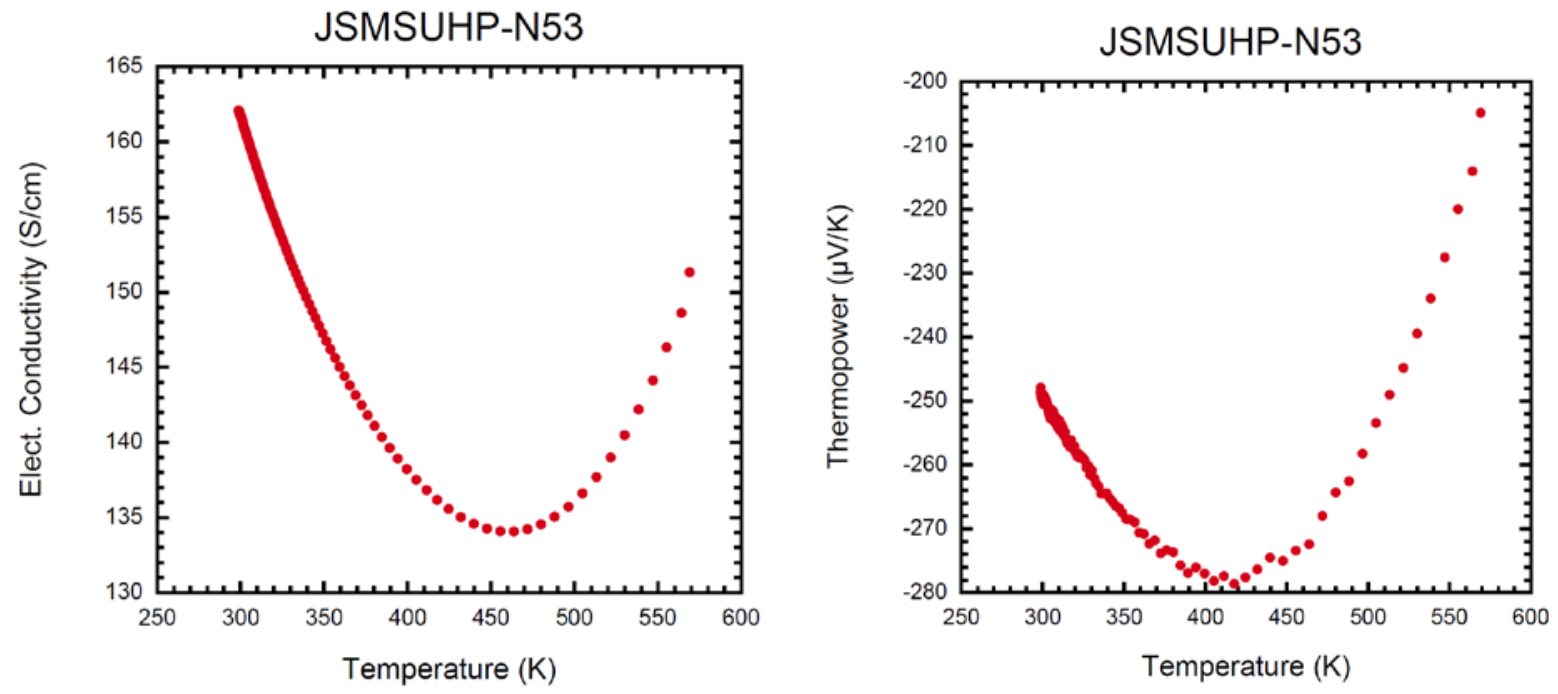

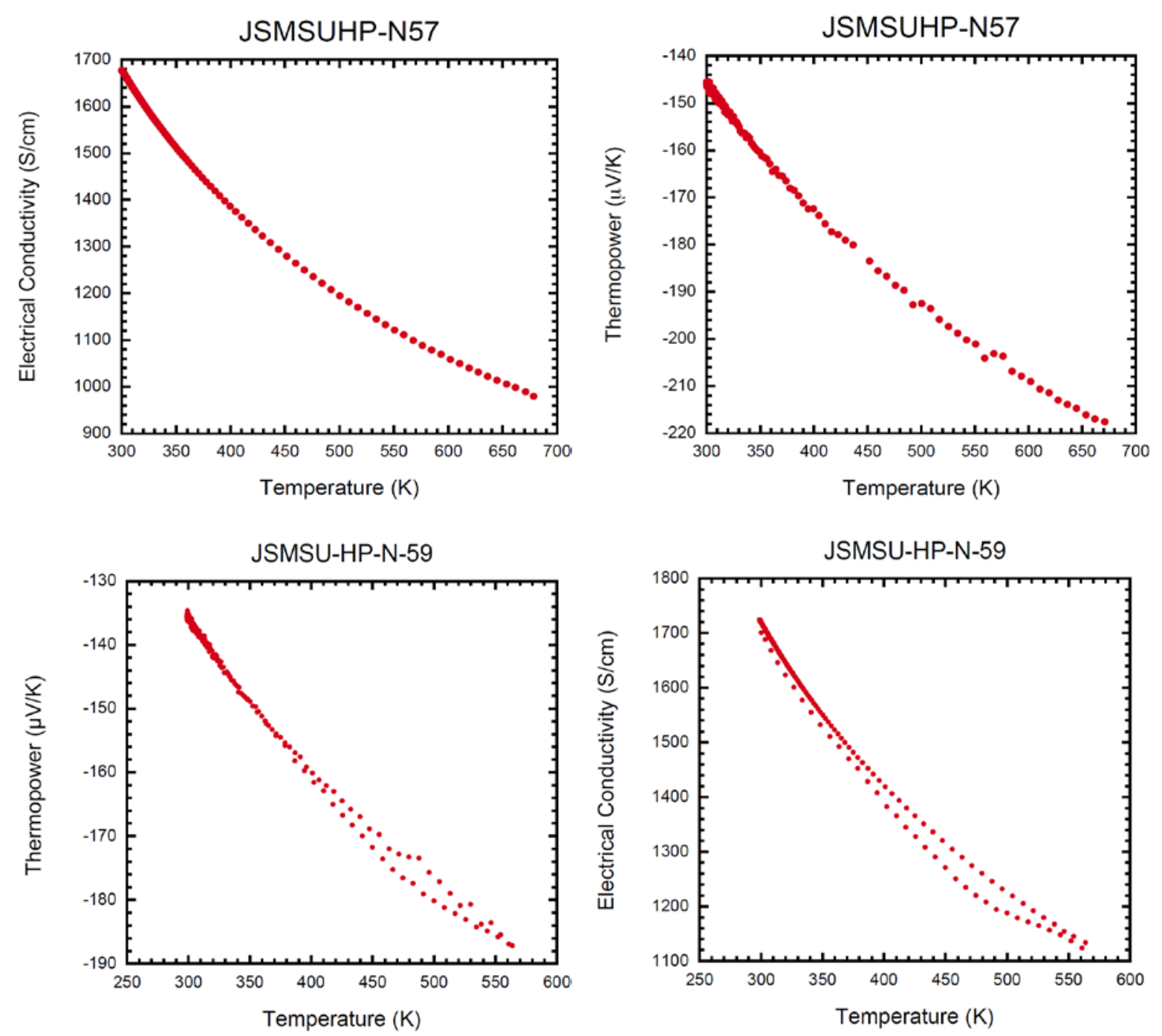

Figure 2.12. N-type skutterudite electrical conductivity and thermopower measurements. 

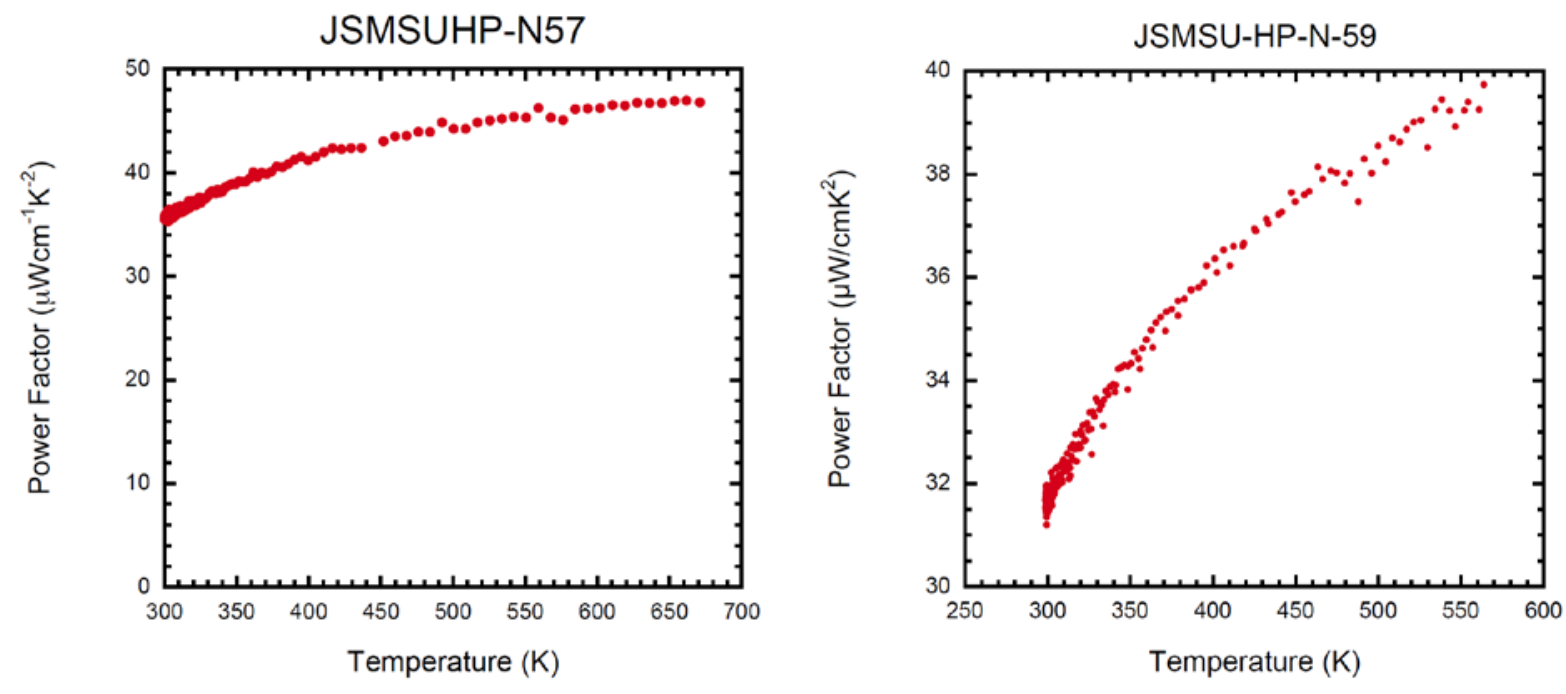

Figure 2.13. N-type skutterudite power factor based on data in Figure 2.12.
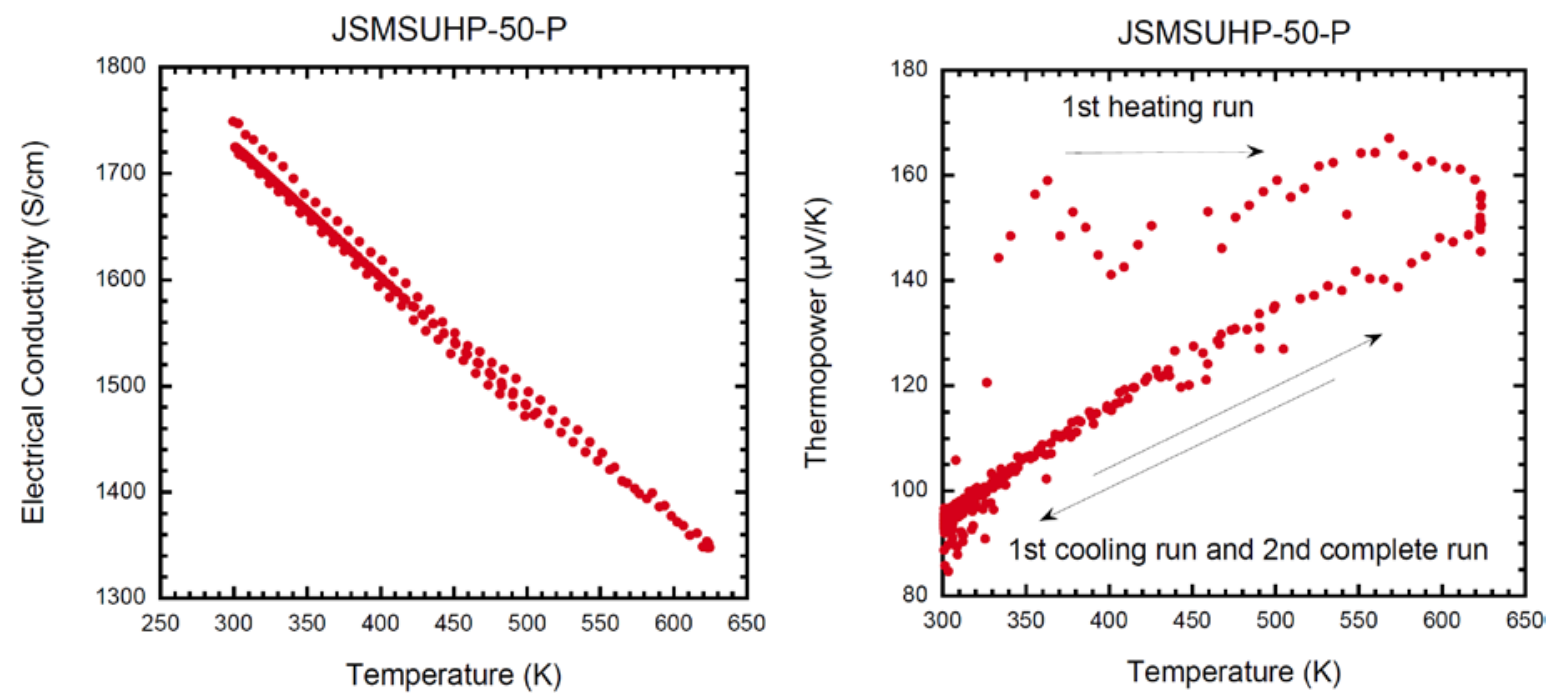

Figure 2.14. P-type skutterudite electrical conductivity and thermopower measurements. 

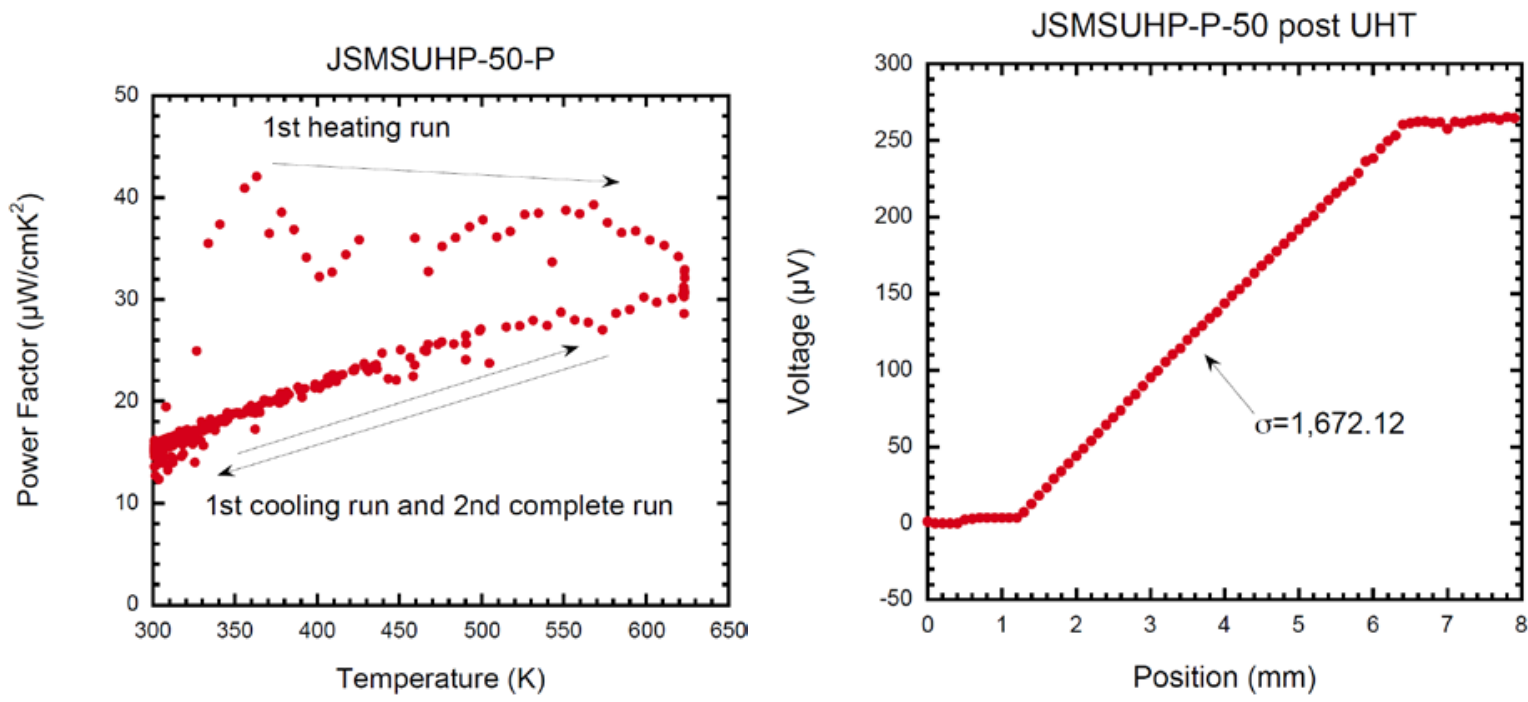

Figure 2.15. P-type skutterudite power factor and voltage scan across the sample and contacts after the temperature dependent measurements were finished.

It maintained low contact resistances after temperature cycling.

Samples were sent to the Thermophysical Research Laboratory (TPRL) at Purdue University for thermal conductivity measurements, the results are shown in Figure 2.16.
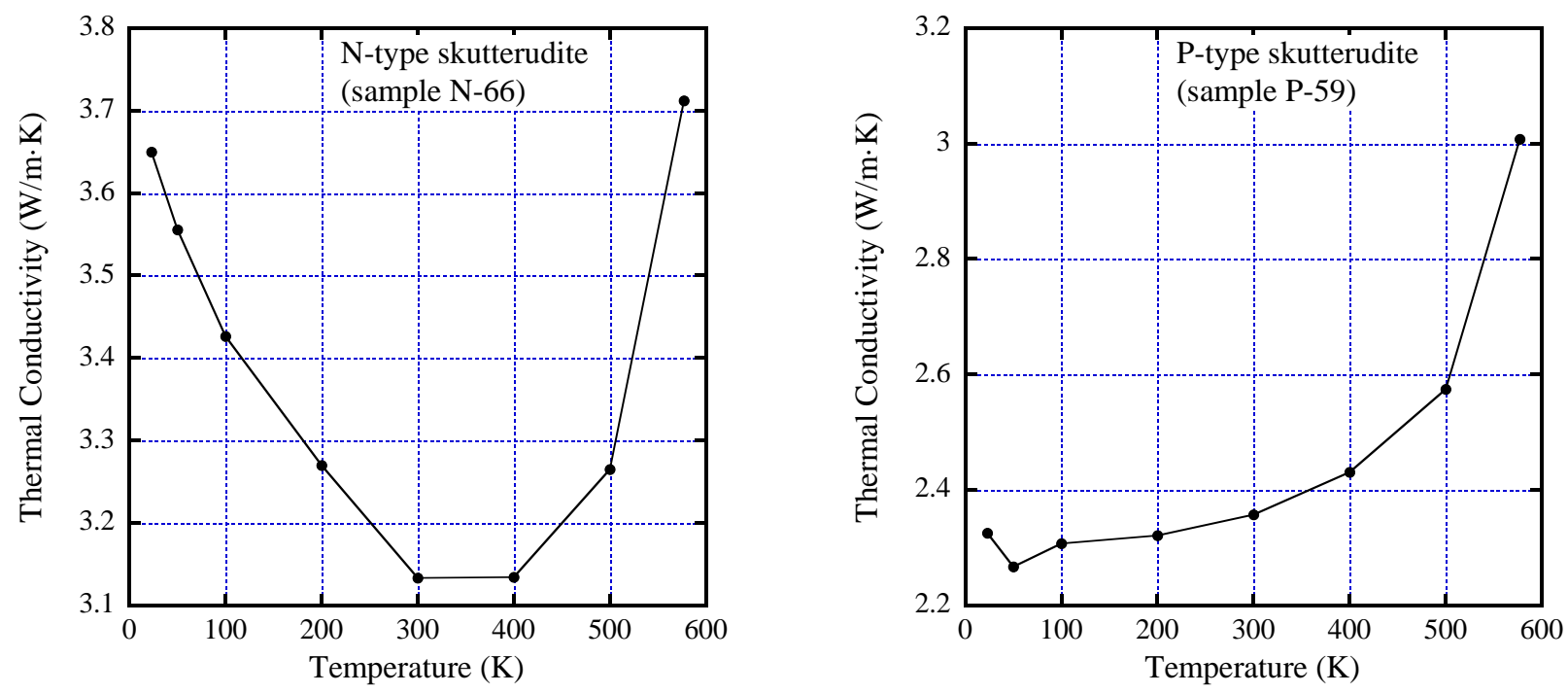

Figure 2.16. N-type and P-type skutterudite thermal conductivity measurements taken at the Thermophysical Research Laboratory (TPRL) at Purdue University. 


\section{Module Development and Fabrication}

\subsection{Couple Fabrication}

The high-temperature couples used in this experiment were constructed by joining two 3.5x3.5x7mm skutterudite legs, one N-type and one P-type with a copper heat exchanger, or hot shoe/contact (Figure 3.1). Applying a temperature gradient between the copper hot shoe and the bottom of the legs causes a current flow and voltage potential through the material. With a temperature gradient of approximately $500^{\circ} \mathrm{C}$ each component will produce around $0.5 \mathrm{~W}$ of power under normal testing conditions.

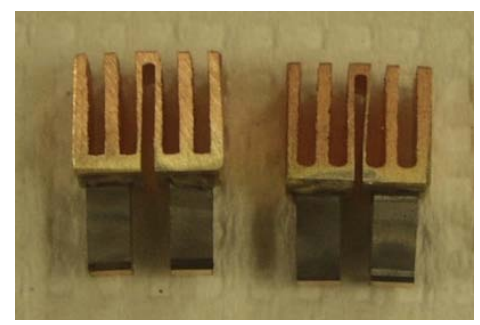

Figure 3.1. Skutterudite N and P-type legs bonded to a copper hot shoe/contact.

\subsection{Module Assembly}

After a batch of skutterudite couples has been fabricated, the couples are used to fabricate modules. A 20 leg module (10 N and P-type couples) is fabricated by soldering a series of 10 couples to a FR-4 module circuit board (shown in Figure 3.2. These modules contain 10 0.5W couples connected in series; thus, each module is capable of producing approximately $5 \mathrm{~W}$ under normal testing conditions. Figure 3.3 shows an old 7 X3.5mm leg module.

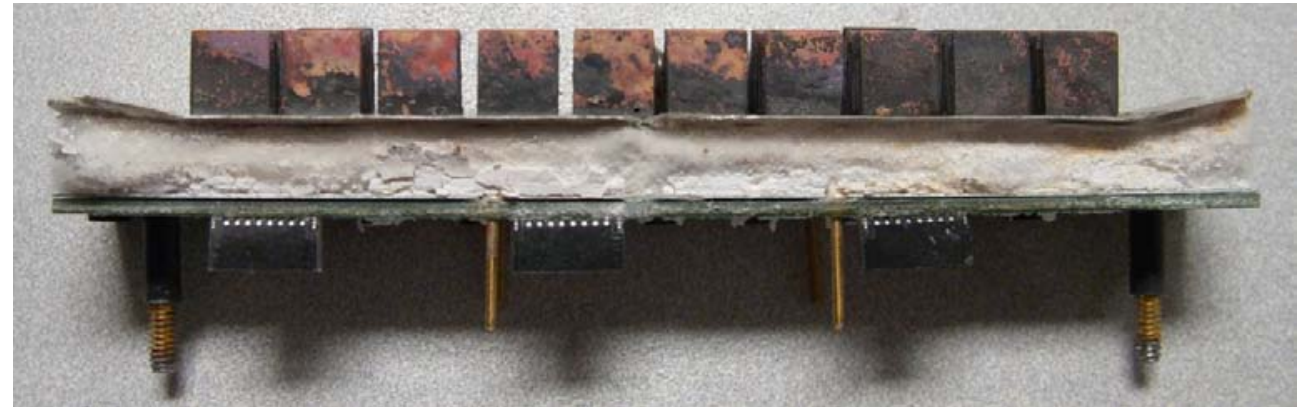

Figure 3.2. A 5W module with aerogel and mica insulation.

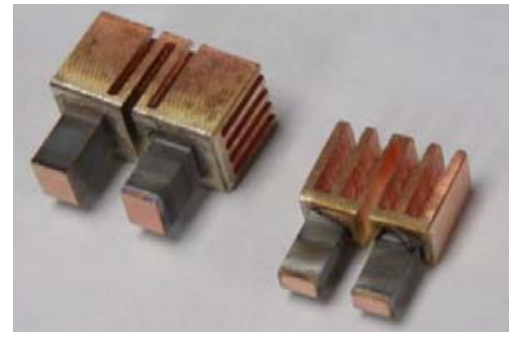

Figure 3.3. Picture of an old 7X3.5mm leg module on left and new $3.5 \times 3.5 \mathrm{~mm}$ leg on right. 
Along with making the couples smaller, we also had to update our base plate and electrical connections to be able to short the electrical circuit between the couples with electrically activated switches (EASs). This was accomplished by using a circuit board as the base plate. We used to maintain soldering pads to transfer heat through the board to keep high heat transfer through the legs, while allowing the EASs to tap into those connections to short out the couples if needed. The circuit board base plate also gave a more robust electrical junction between couples, while reducing parts needed to make those connections. Below, Figure $\mathbf{3 . 4}$ shows an old 5 couple module and a new 10 couple module with a circuit board.

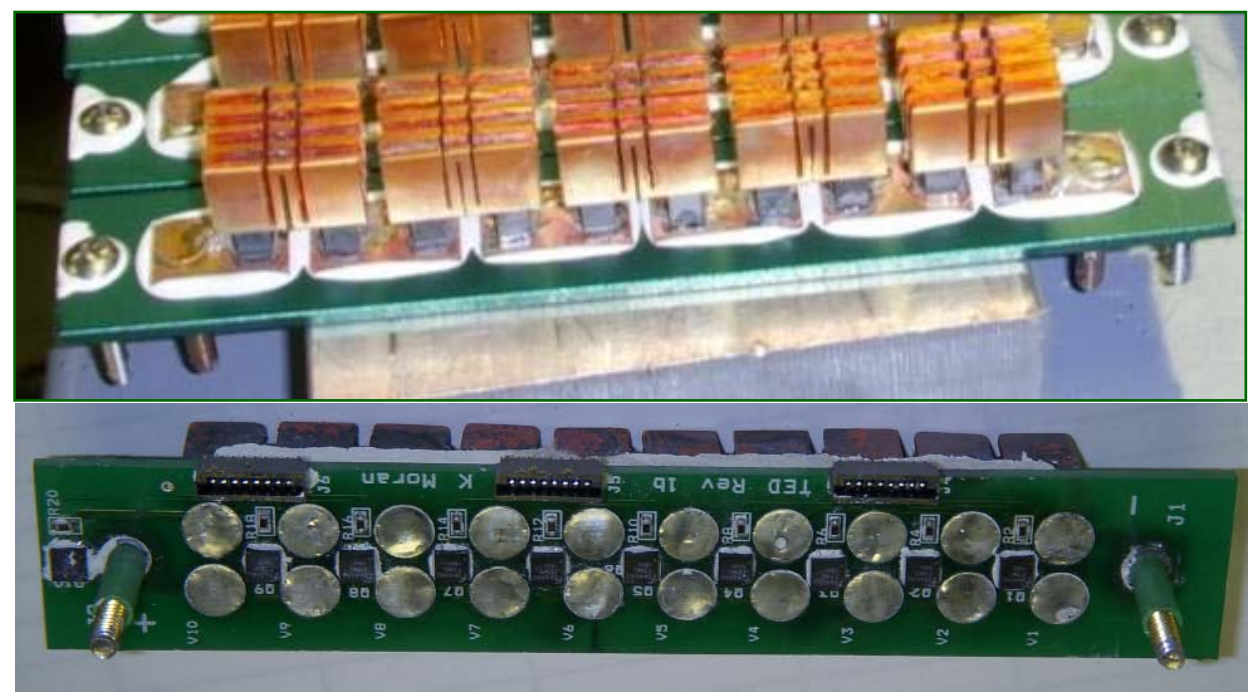

Figure 3.4. Above is an old 5 couple module with aluminum base plate and below is a new 10 couple module with a circuit board as its base plate and new $3.5 \times 3.5 \mathrm{~mm}$ leg.

\subsection{Changes from Previous Fabrication Process}

As research efforts have continued, a number of changes have been made to the fabrication process to improve the performance of the thermoelectric modules. Many of these changes have necessarily stemmed from the reduction in leg size, while others are the result of continuous improvement efforts.

\subsubsection{Metal Foil}

One of our goals has been to incorporate metal foil into the metalized layers of the hot pressed $\mathrm{N}$ and P-type skutterudite (SKD). The purpose of the metal foil is to suppress the sublimation of the antimony $(\mathrm{Sb})$. The layers of each metal and the sequence to which they are loaded in the hot press die were previously shown in Tables 2.1 and 2.2. The metal foil is placed on both sides of the SKD powder for both the $\mathrm{N}$ and P-type material.

Based on the results from bonding, the incorporation of the metal foil into the P-type material is not yet optimized, as failures are still occurring during the bonding process (i.e. material cracking between the metal foil and skutterudite). 


\subsubsection{Round Jig Design}

As higher production volumes rates are envisioned, process repeatability and consistency are increasingly important. Any unnecessary variability in the process should be eliminated. Unnecessary variability should be reduced to a minimum in order to consistently and reliably produce thermoelectric modules that have nearly identical characteristics.

One area that previously had a large margin of variability was the insulation for the bonding station. Current procedures for the bonding station call for a graphite jig to be loaded with skutterudite legs. Brazing foil strips are placed on each leg and each P-leg and N-leg pair are topped with a hot shoe to form a couple to melt the brazing foil, the graphite jig is placed in a Kurt J. Lesker vacuum chamber and secured under a ceramic button heater, which is controlled by a Heatwave Labs temperature controller. The controller uses a feedback thermocouple to ramp the temperature to $750^{\circ} \mathrm{C}$ over a period of 30 minutes. To achieve this temperature while sweeping the vacuum chamber with Argon gas, and to preserve the integrity of the thermocouple attachments, Fiberfrax high alumina ceramic insulation is used to insulate the graphite jig and the ceramic heater from the rest of the vacuum chamber. This insulation comes in two forms: a high density inflexible "block" form and lower density flexible "blanket" form.

To insulate the jig and heater, four square, ceramic insulation "blocks" were cut for the sides of the jig; the bottom of the jig sat on a copper cooling plate. To insulate the top of the jig, (where the ceramic heater was located) strips of the ceramic insulation in blanket form were simply "stuffed" around the heater. Both the side insulating blocks and the top insulating strips expose the heating cycle to an undesirable level of variability. During some bonding runs it was suspected that the insulation was not sufficient to achieve the even heating of the jig necessary to produce high bonding success rates. The solution was to add more insulation to the top of the graphite jig. In addition, the side insulating blocks would periodically fall over during the preheating vacuum cycle. To prevent this, a thick wire was used to encircle the blocks. These solutions, however, were crude and the level of variability was still higher than desired.

To reduce this variability, a new, circular jig design was developed. Whereas the previous square design required four spring-loaded supporting bars to accommodate the thermal expansion, the new circular design required only one supporting bar. Because of this it was possible to cut two doughnut-shaped insulation blocks to completely encircle the graphite jig. In addition, the uninhibited access to the ceramic heater allowed for the installation of two insulation blocks to encircle the heater. Only minimal insulating variability remains as a few strips of the blanket form of the insulation are used to encircle the area where the ceramic heater attaches to the threaded compression mount.

To test the effectiveness of the insulation with the circular jig, an experiment was conducted to determine the maximum temperature of the space enclosed by the insulation versus the maximum temperature of the space outside the insulation. Thermocouples were set up to measure the temperature of the graphite jig as well as the temperature of 3 locations outside the insulation. The usual heating ramp cycle was then run and the temperatures recorded every 5 seconds. The maximum temperature values of the entire cycle were compared to evaluate the effectiveness of the insulation. This experiment was performed twice with the square jig and insulation setup, and twice with the circular jig and setup. The results of the experiment indicated 
that the circular setup is just as effective as the square setup at insulating the jig and heater from the rest of the chamber.

\subsubsection{Tracking and Measurements}

As advancements are made and more ambitious power goals are set in the area of thermoelectric generator testing, it becomes desirable to gain greater insight into the process by which the thermoelectric modules are constructed. A certain level of understanding is gained when the performance of the skutterudite material is measured by testing the thermoelectric modules in a generator. However, a greater level of understanding could be attained if the performance of the skutterudite material could be measured at every step of the preparation process. We could discover which steps result in considerable performance degradation.

One indicator of skutterudite performance is the electrical resistivity of the material. As with any thermoelectric material, it is desirable for the material to have a high value of thermal resistivity and a low value of electrical resistivity in order to maximize power output when exposed to a given temperature gradient. Previously, the electrical resistance of the material was first measured after soldering 5 couples together into a module. The electrical resistance of each individual leg and each couple was never measured. Thus, it was impossible to note the performance of an individual leg or couple, but only the performance of a 10 leg module. Not surprisingly, the electrical resistivity varied significantly from one module to the next (as much as $40 \%$ in some cases).

To address this, two new testing jigs were fabricated. One tested the electrical resistance of an individual leg and one tested the electrical resistance of a two-leg couple. The operating method of both of these jigs is the same. Two copper leads provide the electrical contact between the legs and a Kepco power supply. One or both of the copper leads are spring loaded to secure the leg/couple in the jig. The voltage drop across the leg/couple is measured with a multi-meter while applying a current of 1 amp with the power supply. These two values are then used to calculate the electrical resistance of the leg/couple.

The procedure for creating modules was updated to include measuring the electrical resistance of the legs before bonding into couples and then measuring the electrical resistance of the couples before soldering into modules. In addition, the updated procedures also included measuring of the electrical resistance of the modules after being soldered, after being glued to the base plate and after the addition of the aerogel insulation.

Under these new procedures the electrical resistance of N-type legs that were cut from the same puck varied considerably, (as much as $50 \%$ in some cases). The electrical resistance of P-legs cut from the same puck varied only slightly.

To investigate the homogeneity of the pucks a new method of leg tracking and documenting was established. Under this new method, each puck is marked to note the orientation in the hot press. Using this mark, the pucks are then cut using the following diagram (Figure 3.5) to assign a number to each of the legs. Each leg is then placed into a numbered glass container to be washed and cleaned. The legs are then moved into new containers and their electrical resistivity is measured and recorded. 


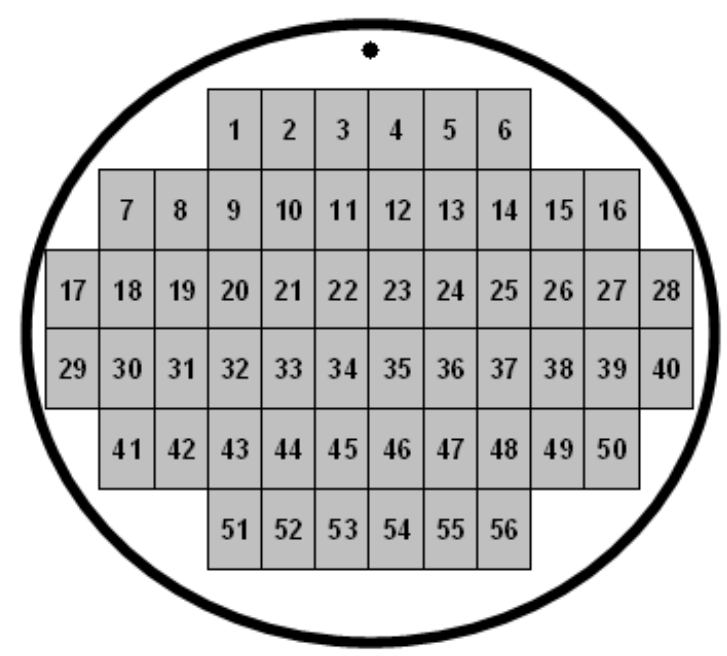

Figure 3.5. Leg tracking and documenting from hot pressed pucks.

The next steps to investigate the puck homogeneity include etching a number onto the hot shoes in order to track the legs during and after bonding. This will also prove useful in determining whether there is a correlation between electrical resistance in the leg and the bonding success rate. In addition, the base plates of the modules will be numbered as well, so that it will be possible to track an individual leg and find its exact location in a module.

Several N and P-type pucks have been documented under the new tracking method (Figure 3.6). Typically the electrical resistances of the P-type legs were very consistent; varying by no more than 0.2 milliohms, while the electrical resistances of the N-type legs varied considerably, up to 0.6 milliohms. In addition, the legs that came from the edges of the puck had higher resistance values than the legs that came from the center of the puck. 


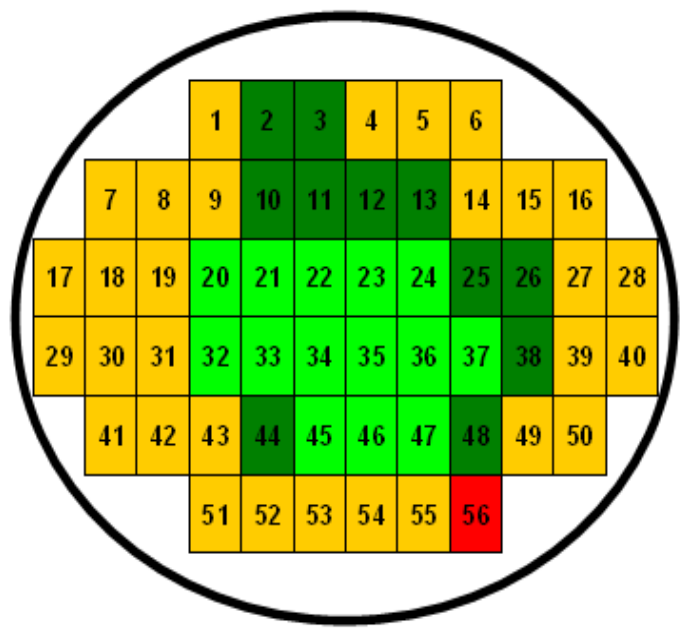

N-type: $1^{\text {st }}$ Measurement

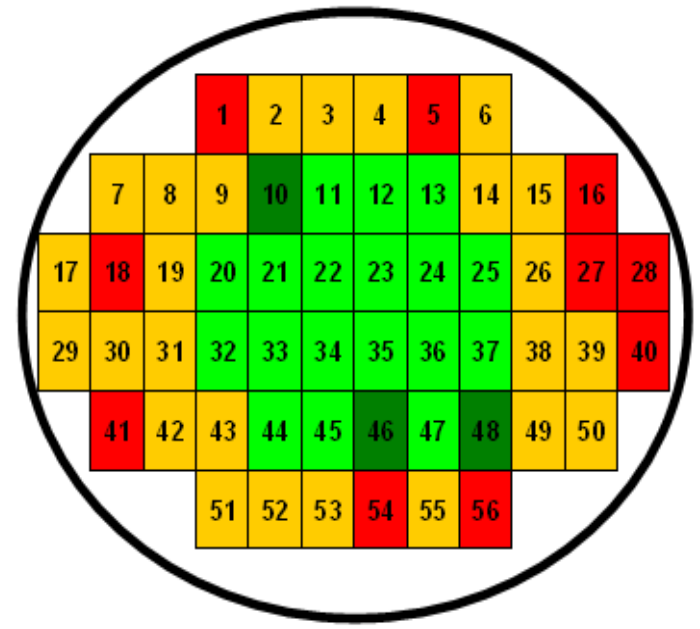

N-type: $2^{\text {nd }}$ Measurement

Green Values: Below or equal to 1 Standard Deviation

Dark Green Values: Between Avg Resistance Value and 1 Standard Deviation Below

Orange Values: Between Avg Resistance Value and 1 Standard Deviation Above

Red Values: Above 1 Standard Deviation

Figure 3.6. Measured electrical resistance of N-type legs.

\subsubsection{Brazing Agent}

Efforts to improve the couple fabrication process were conducted. Different types of brazing agents were investigated to include CuAg-based foils and paste. The foil brazed couples were bonded at different temperatures and dwell times to cause full or partial melting of the braze. The braze paste enabled bonding at slightly lower temperature. The couples were then cycled in the couple testing station. All of the couples were heated from room temperature to $656^{\circ} \mathrm{C}$ in 30 minutes, held at $656 \mathrm{C}$ for 15 minutes and cooled to room temperature in 30 minutes. This thermal cycle was repeated 9 times. The cold side temperature was maintained at $\sim 25^{0} \mathrm{C}$ throughout the test. The data (Figure 3.7) shows the total couple power output and resistance as a function of cycle number. The couples that were bonded with the braze paste had the highest power output (nominally $1 \mathrm{Watt}$ ), while the fully melted braze foils had the lowest power output (nominally 0.82 Watts). The reason for the difference in power outputs might be related to the temperature during bonding, as the braze paste samples were bonded at lower temperature, while the braze foil samples were heated at higher temperatures. The higher temperature bonding could have caused the thermoelectric materials to degrade slightly, thus reducing the power output. Interestingly, the braze foil couples had lower resistance than the braze paste couples. This is counter to what would be expected, since the power output and resistance are inversely related, i.e. the lower the resistance the higher the power output. This again could be explained by the fact that exposure to the higher temperatures caused the thermoelectric material to degrade. Typically, when the skutterudite degrades it becomes more of a metallic conductor, thus reducing the resistance. 

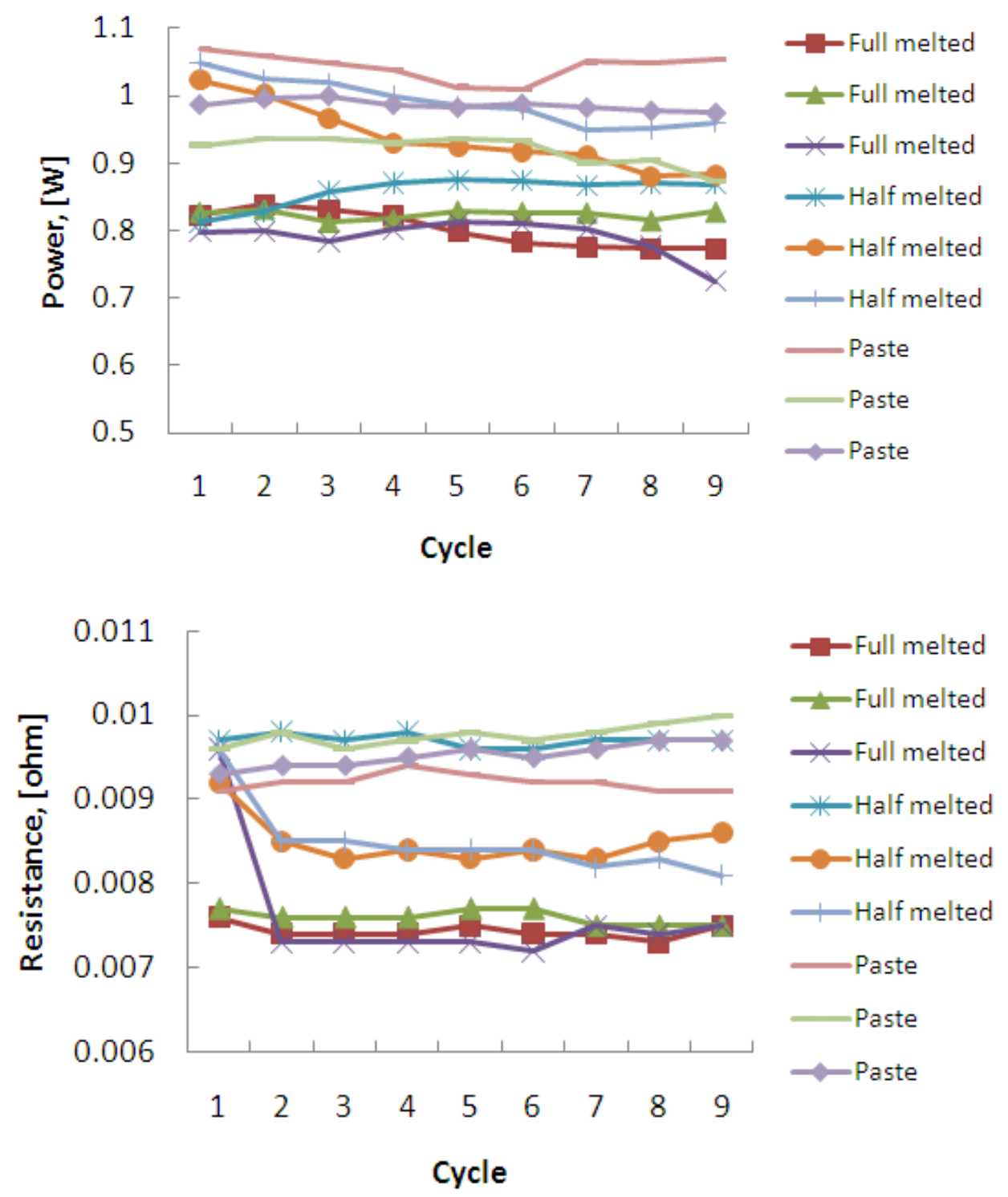

Figure 3.7. The output power (top plot) and electrical resistance (bottom plot) as a function of thermal cycle from $25^{\circ} \mathrm{C}$ to $656^{\circ} \mathrm{C}$.

\subsubsection{New Hot Shoe Design}

In our initial design of the TEG, we tested multiple couple designs and TEG flow configurations to produce optimum output from our thermoelectric material. After a few tests we concluded that our hot shoe design needed to capture more heat. The hot shoe was redesigned from a flat hot shoe to a fin type hot shoe that had more than 20 times the heat sink area (shown in Figure 3.8). We also made the base design of the 10 leg module more robust by mounting them on a base plate that could be easily bolted in and out of the TEG. 

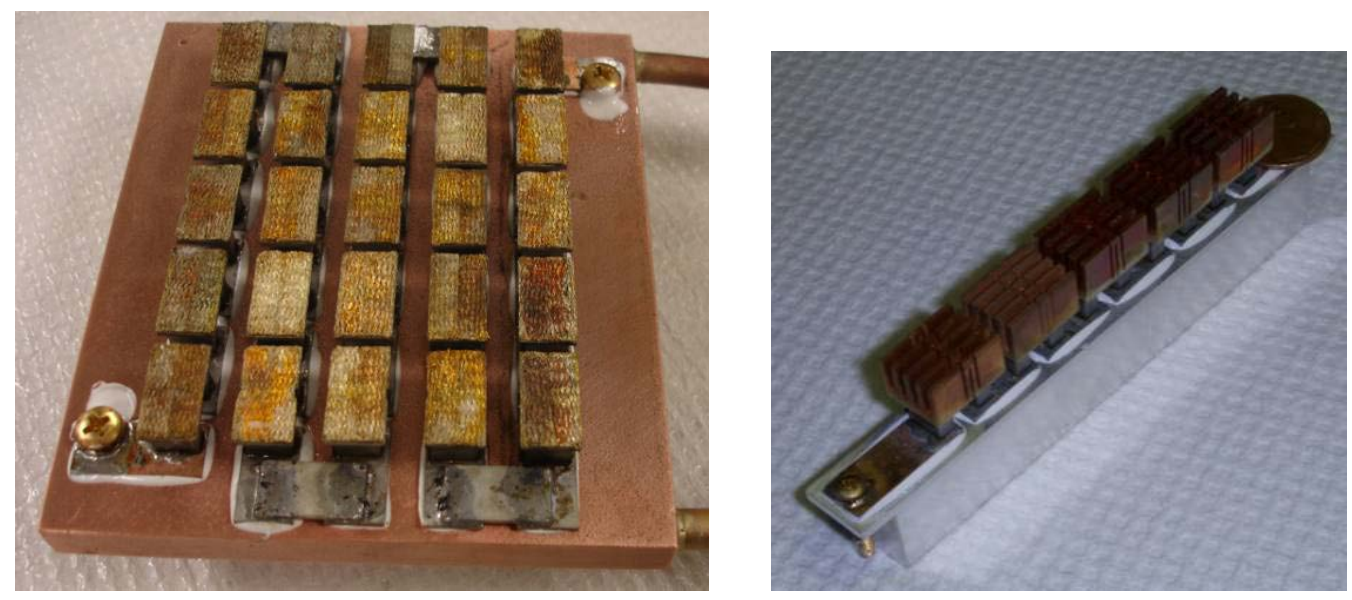

Figure 3.8. TEG with flat hot shoe design compared to a 10 leg module with heat sink hot shoe.

With the new, smaller leg design, the hot shoe was redesigned as well. The positive features of the finned hot shoe design (such as expanded surface area and improved flexibility) were also implemented into the new hot shoe design. As seen in Figure 3.9 below, the new hot shoe design allows for different amounts of thermal expansion between the $\mathrm{N}$-leg and the P-leg, while utilizing the fin structure to improve heat transfer rates.

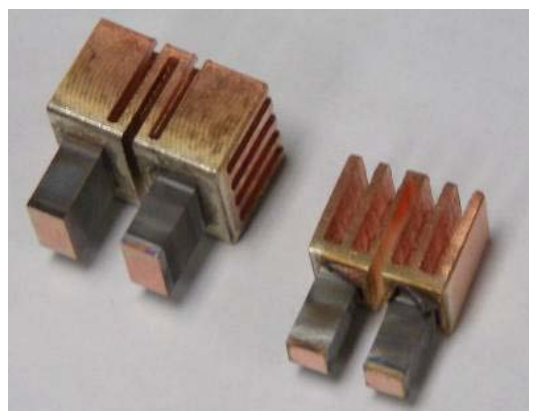

Figure 3.9. New hot shoe design.

\subsection{EDM}

There had been some concern that the sharp corners on the rectangular legs can lead to high mechanical stresses in the legs, resulting in cracking and failure of the module. To explore potential solutions to this concern, several $\mathrm{N}$ and P-type skutterudite pucks were cut into round legs using Electrical Discharge Machining (EDM). The results for the N-type were encouraging; however, the P-type in most cases did not survive the process. The results from a 2 inch N-type skutterudite puck are shown in Figure 3.10. 


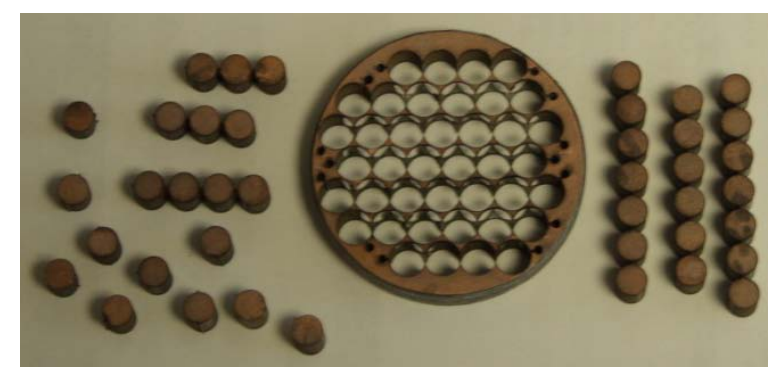

Figure 3.10. Circular legs cut from a 2 inch N-type skutterudite puck using EDM.

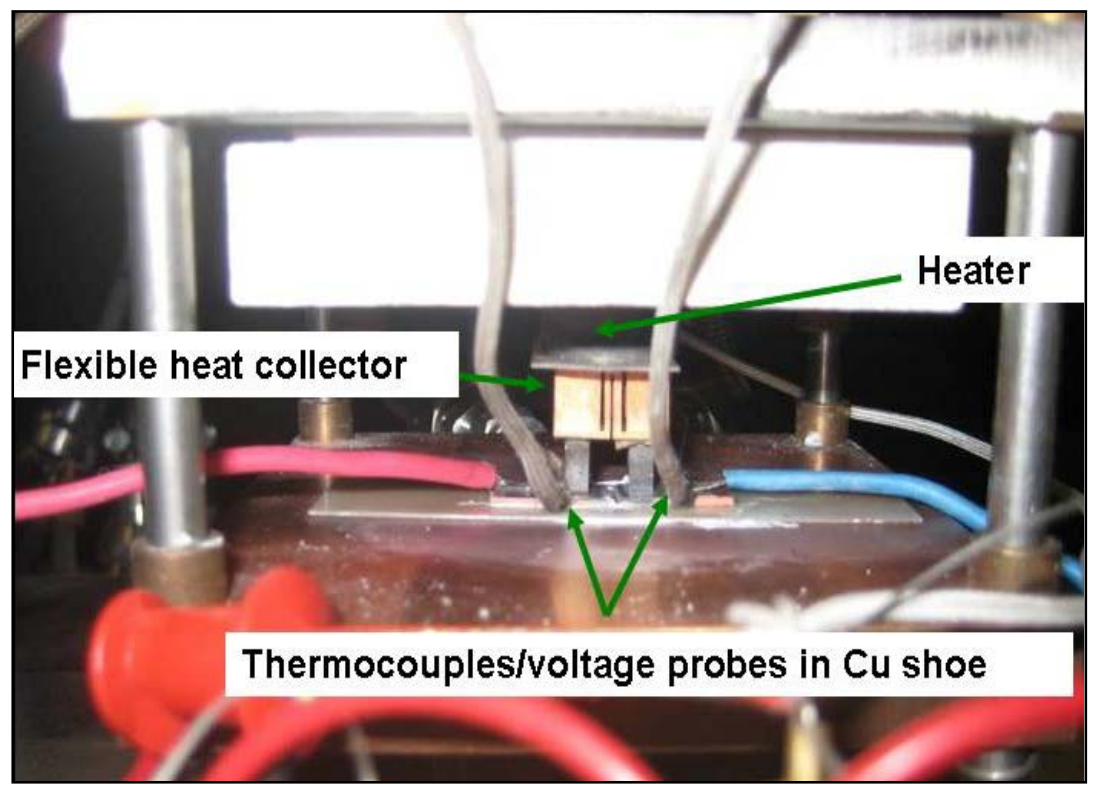

Figure 3.11. Couples were tested in a belljar and were instrumented to enable power output measurements on the individual legs and collectively as a couple.

When using the EDM cutting technique, a total of 40 legs were obtained from the puck compared to 56 when using the diamond saw. The contact surface area of the circular legs was equal to the rectangular legs. The P-type material cut well using the EDM technique. However, the material cracked between the metal foil and skutterudite layers. A finite element analysis has shown that the circular design helps reduce the stress concentrations by eliminating sharp corners. The circular design has not been adopted into the fabrication procedures due to these manufacturing difficulties.

\subsection{Individual Couple Testing}

To assess individual couple performance couples were tested in bell jar stations with conductively heated hot shoes and a cold plate. Thermocouples were instrumented into the hot and cold shoes. This also acted as voltage probes to measure the voltage and resistance of each leg individually and together (Figure 3.11). 
The power output was measured using the same heating rates that are used in our TEG test bed, i.e. heating from room temperature to $650 \mathrm{C}$ in 30 minutes or $22 \mathrm{C} / \mathrm{min}$ and dwelling for 15 minutes, then cooling at the same rate. A constant current was passed and matched to the expected current (19.1 amps) at peak power and the voltage was measured. Essentially multiplying 19.1 amps times the leg/couple voltage gives the power output in watts (Figure 3.12), as a function of time at a particular thermal gradient. The hot and individual cold side temperatures are also plotted in the same plot (Figure 3.12). It is apparent that by replacing the old formulation N-type legs with the new formulation results in an estimated $166 \%$ increase in power output for approximately the same thermal gradient. Unicouples made with the old formulation delivered 1.2Watts, on average, while the new formulation $\mathrm{N}$-type couples deliver 2.0Watts. This increase is expected to result in a similar increase in the TEG performance. The data in shown in Figure 3.12 also provides preliminary results on the thermal cycling behavior of our couples. This first of 10 thermal cycles are shown. It is important to note that the couple was able to survive reasonably well; it did not catastrophically fail upon cycling. The skutterudite material and the metallization used is believed to be reasonably robust, thus is able to withstand the preliminary cycling tests. There are some signs of degradation in the N-type legs; more so than in the P-type leg. Post cycling test analysis indicates that there was some sublimation and bond integrity degradation on the hot side of the N-type leg.

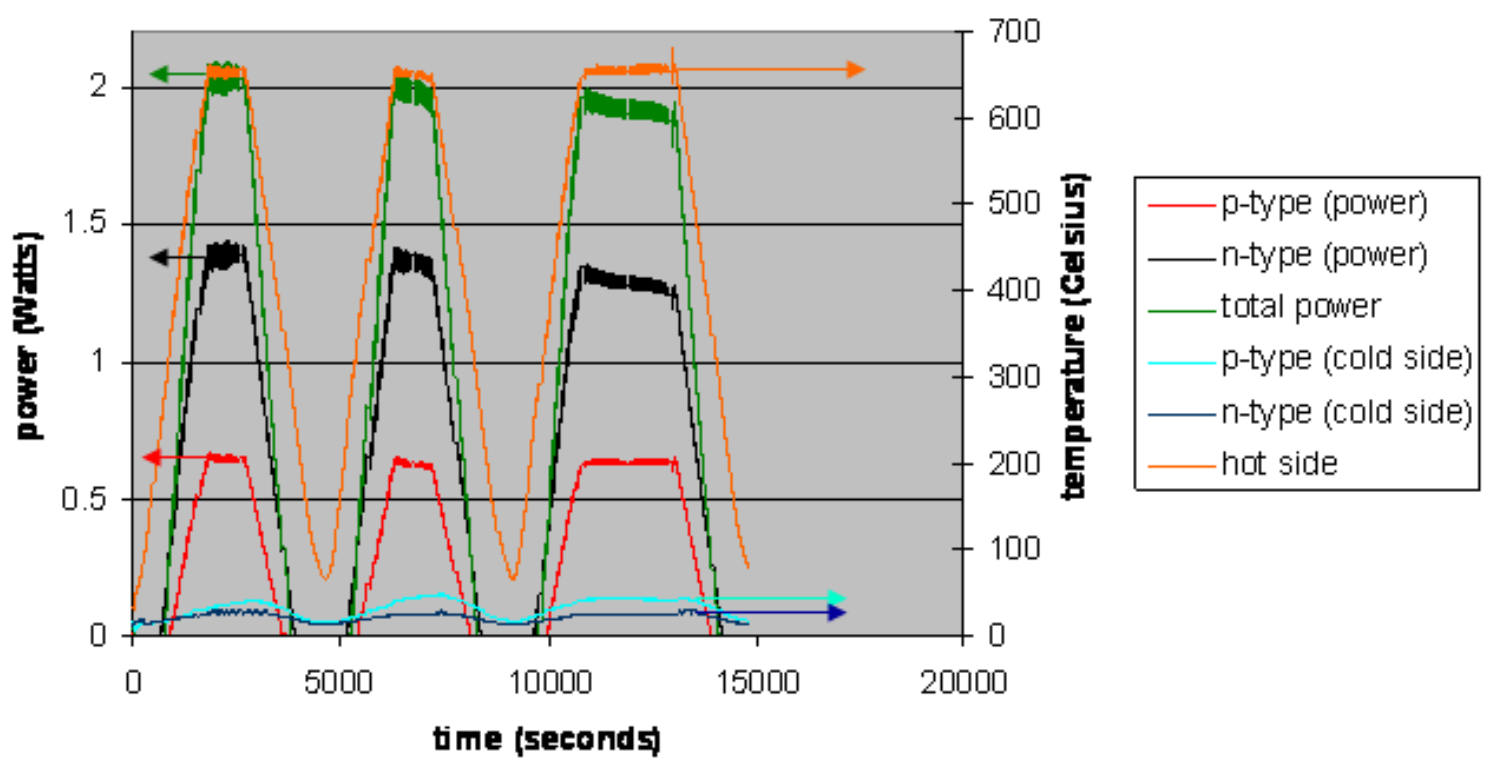

Figure 3.12. Power output data for couples made using the new $\mathrm{N}$-type formulation legs.

Several other tests were conducted on similar couples. The cycling tests indicate that the preliminary couples survive the cycling tests reasonably well. The couple that provided data (as shown in Figure 3.12) was cycled a total of 10 times. When comparing the $10^{\text {th }}$ cycle to the $1^{\text {st }}$ cycle the power output decreased by $20 \%$. 
The individual couple testing was performed on the new smaller leg design as well. Once the hot side reached temperature, the current was incremented to derive a power curve, from which the maximum power was calculated. Similar to the larger leg design, the couples survived 9-10 cycles with fairly low degradation. While individual couple testing resulted in power levels of $1 \mathrm{~W}$ per couple, generator testing results in power levels of only $\sim 0.5 \mathrm{~W}$ per couple. Nevertheless, consistently demonstrating 1 Watt per couple over 9 cycles should result in the next generation of TEG capable of delivering well over 100 Watts for multiple cycles.

\section{Material Technology}

\subsection{Functionally Graded Material Methodology in Thermoelectric Module Development}

In this section, the Functionally Graded Material (FGM) methodology is investigated on an arbitrary cuboid (MSU has filed a patent disclosure on this technology). The aim is to understand its applications and subsequent benefits if applied to the thermoelectric (TE) module development. The FGM method was developed to meet component requirements for material performance that varies within the component. Abrupt transitions in materials composition and properties, within a component, will result in high local stress concentrations. With FGM these stress concentrations can be greatly reduced, thus increasing performance.

In TE applications, FGM can improve (smooth) temperature distribution throughout the modules. This, in conjunction with the smoother transition of mechanical properties through the graded layers, reduces the magnitude of thermal stresses. The locations where these stresses peak can be controlled and the onset of plastic yielding and failure can be delayed. The severe stress concentrations arising at the intersections between free edges and material interfaces can also be suppressed. The strength of the bond between dissimilar materials can be increased with the introduction of graded materials, hence, minimizing the driving forces for crack initiation and growth along and across the interface. Finally, specifically to TE applications, FGM can be used to maximize the module efficiency. As the module experiences a temperature gradient, the materials comprising it can be graded in such a way as to maximize the figure of merit (Z) at each location, resulting in maximum thermoelectric conversion.

In order to develop a preliminary understanding of the methodology and explore its potentials a test problem was analyzed. A cuboid representative of the TE module leg geometry was modeled in Abaqus. The cuboid is comprised of the two key materials used in the modules developed at Michigan State University. These two materials demonstrated high stress concentrations at their interfaces. This would frequently result in failures during testing. A schematic of the different cuboid compositions analyzed are shown in Figure 4.1. 

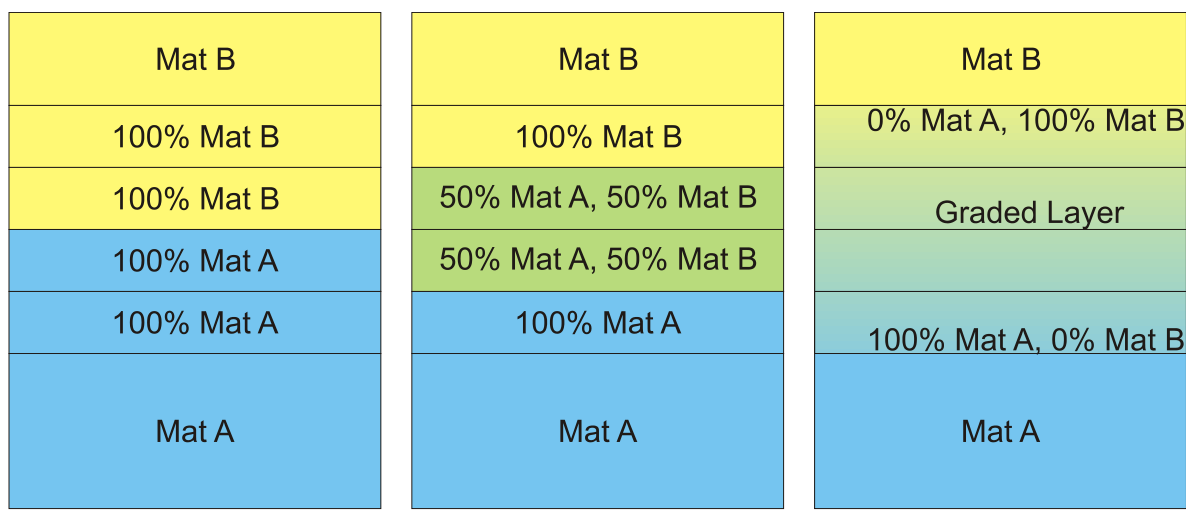

Figure 4.1. 2D schematic of layers used in the numerical experiment.

A comparison is made between pure material layers, a discrete layer comprised of $50 \%$ of each material, and a graded layer. At this initial stage a linear gradient model was used and the resulting material properties of the graded material are assumed to obey the rule of mixtures.

Figure 4.2 through Figure 4.4 show the results obtained using finite element analysis. The graded layer shows superiority in thermo-mechanical properties. A module with a graded layer will experience a smoother temperature profile across the interface (Figure 4.2), as well as lower stress concentrations (Figure 4.3 and Figure 4.4). These enhanced properties will ensure high performance and robustness. The 50-50 layer, which has already been implemented in test modules, shows enhanced properties compared to the pure layers. The interfaces with the pure materials (Figure 4.3 - Mat A at the bottom and Mat B at the top) show abrupt changes in stress concentrations, which can potentially result in failures over prolonged operating times.

These preliminary results demonstrate clearly that a functionally graded TE module design would yield smoother temperature distributions across the graded layer, as well as a reduction in stresses. These enhanced thermo-mechanical properties of the graded layer increase robustness of the module during operation, as well as fatigue resistance, and resulting in an extended lifespan. These observations have already been verified with experiments using a 50-50 layer. A functionally graded layer results in more robust modules. Choosing the right gradient models for this application, as well as choosing the right model to derive the enhanced, mixed material properties requires extensive experimental and numerical investigations. Pursuing this will shed light and develop scientific understanding for the whole process as applied to TE module development. Findings from such research will yield benefits across the whole thermoelectric industry and therefore expediting development of highly robust and efficient modules. 


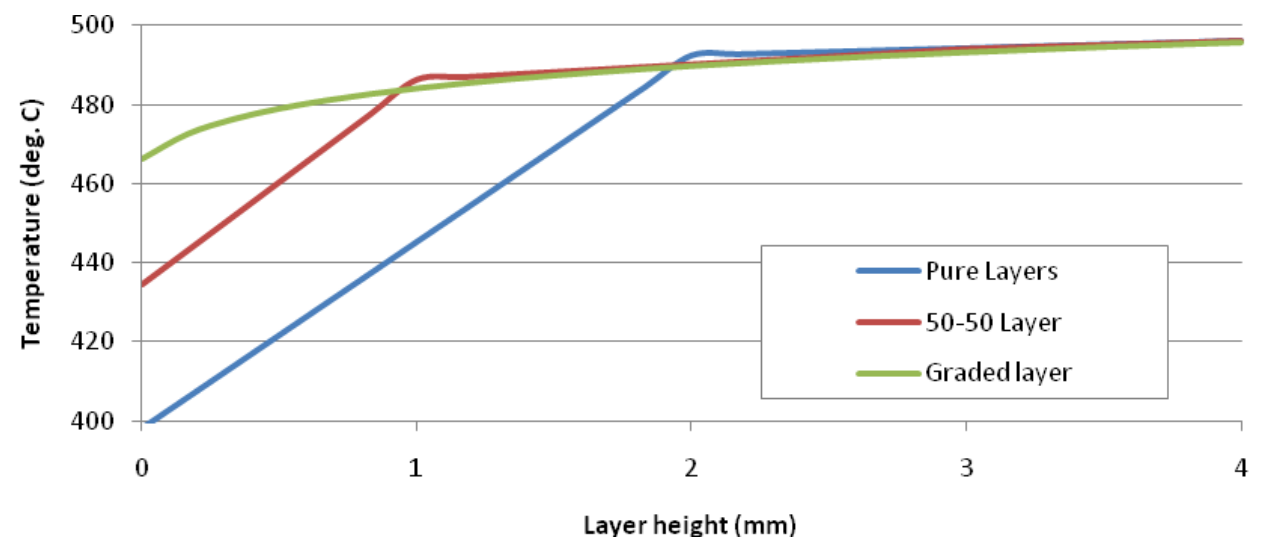

Figure 4.2. Temperature distribution at the center line of layers 1-4.

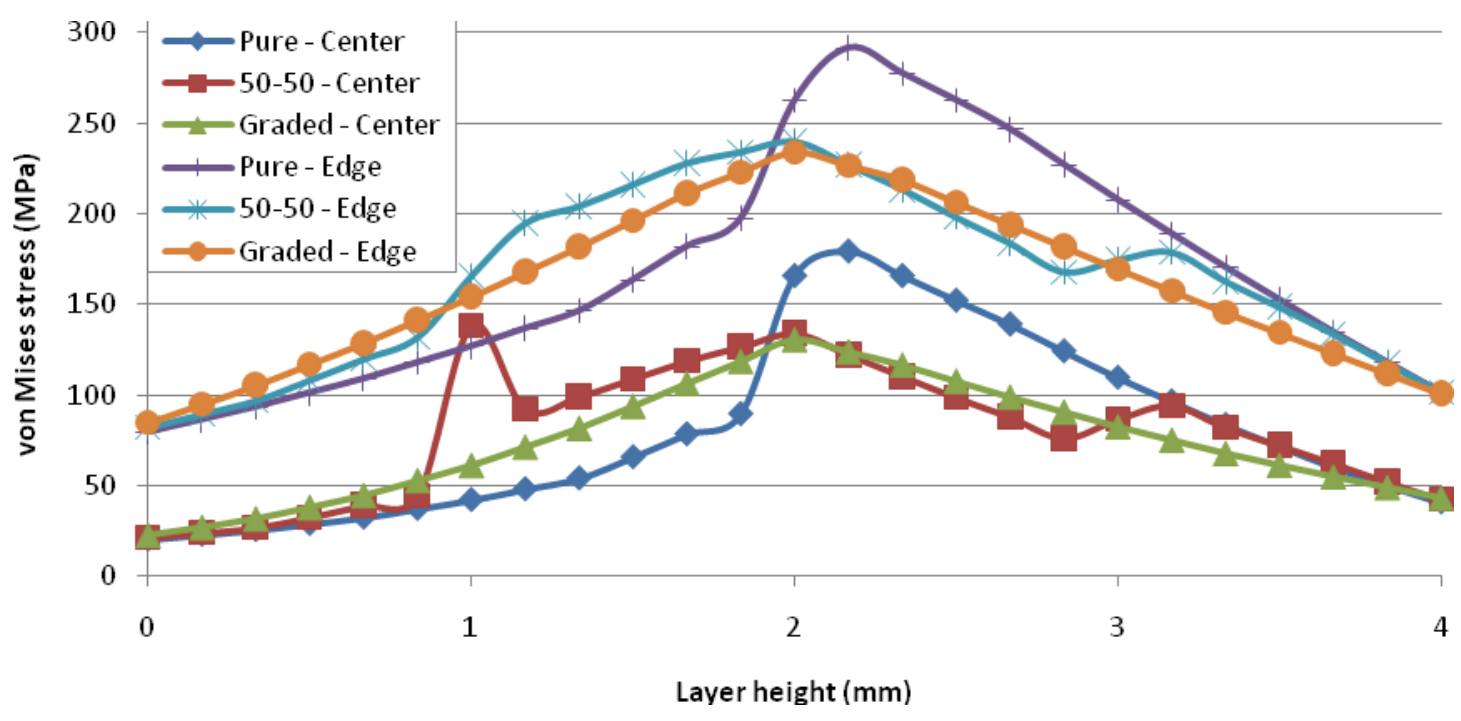

Figure 4.3. Stress distribution at the center line and edge of layers 1-4. 

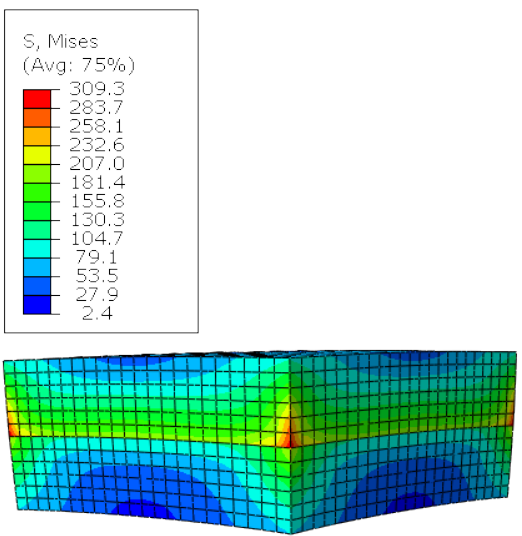
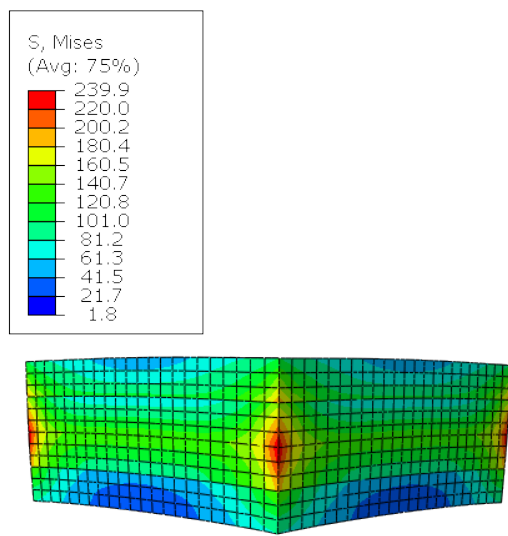
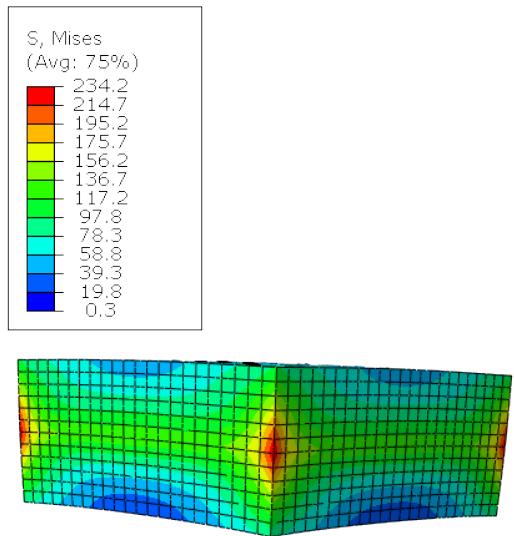

Figure 4.4. Stress distributions at layers 1 through 4, pure layers at the left, 50-50 layer in the middle, and graded layer at the right.

\section{Generator Designs and Results}

Over the course of this project 3 generations of thermoelectric generators have been used to test the couples and modules. This section covers each of these generations as well as the highlights from the test results.

\subsection{Generation 2 (Round Design)}

The first 100W generator included a square copper section that could house 20, 5 couple thermoelectric modules and could produce approximately 100 watts. It was made of a square section for ease in manufacturing and assembly. We used this generator testbed to improve the design of the couples that make up a module and to gain an understanding of the heat flow through the thermoelectric material. After module improvement and further testing it was determined that an improved heat distribution system was required. This prompted the design and construction of round generator housing. Within the square generator the modules in the corners of the generator produced less power than those in the middle. The air flow and the air distribution in the corners were inadequate. Making the generator housing round corrected this problem. The generation 2, round generators can be seen in Figure 5.1. From the tests that were conducted with the square copper, techniques were refined that improved the assembly, manufacturing, and performance of the device. This included a modular cooling plate design for each thermoelectric module to make it easier to replace broken or damaged modules. Making the housing out of aluminum and anodizing it for electrical isolation prevented the module from shorting out on the housing. One $100 \mathrm{~W}$ test was conducted with this design. The results can be found in Table 5.1. 

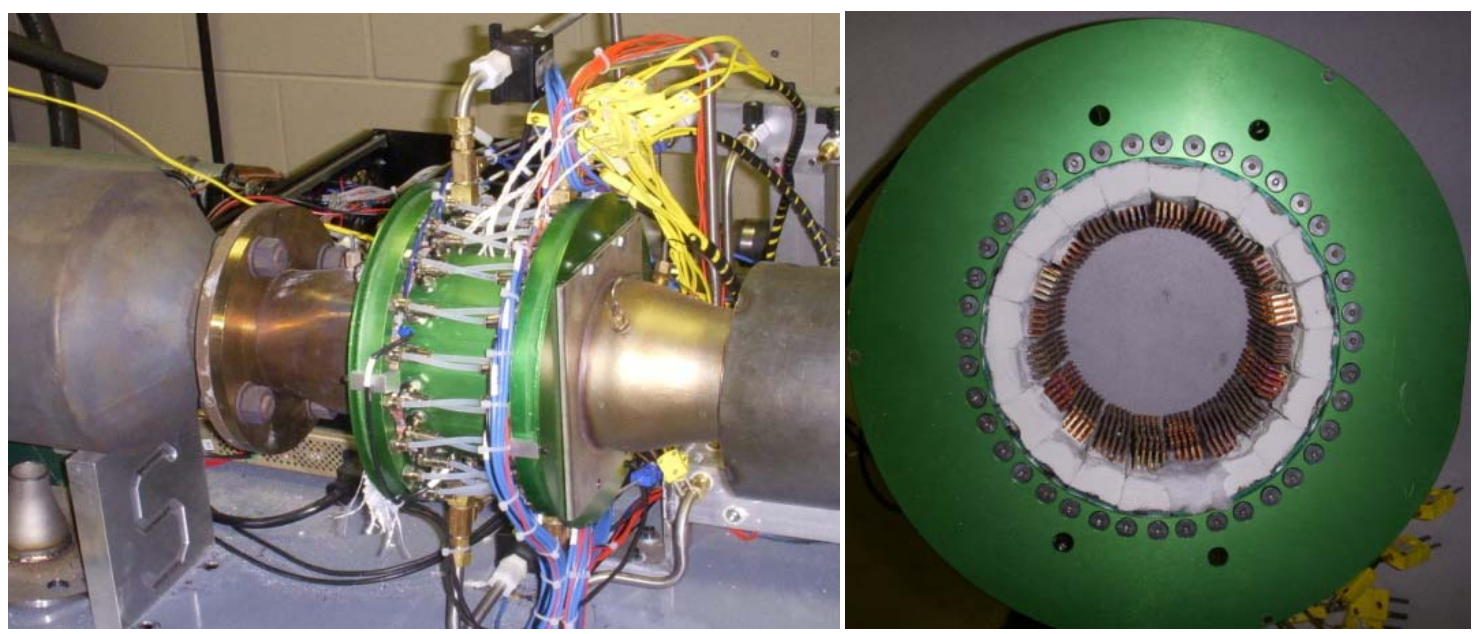

Figure 5.1. Generation 2 MSU thermoelectric 100 watt generator.

Table 5.1. Results from modular cooling plate design for each thermoelectric module.

\begin{tabular}{|c|c|c|c|c|}
\hline Run \# & $\begin{array}{c}\text { Max } \\
\Delta \mathbf{T}\end{array}$ & $\begin{array}{c}\text { Peak Power } \\
\text { (Same Amp) }\end{array}$ & $\begin{array}{c}\text { Peak Power } \\
\text { (Diff Amp) }\end{array}$ & $\begin{array}{c}\text { \# Performed Below } \\
\text { Expectations }\end{array}$ \\
\hline $100 \mathrm{~W}-1$ & $495^{\circ} \mathrm{C}$ & $45.0 \mathrm{~W}$ & $69.8 \mathrm{~W}$ & 7 \\
\hline
\end{tabular}

\subsection{Generation 3 (Round Design)}

Incorporating CBT into the module architecture required several changes to the generator design. The round design, with 2 cooling plate caps, was modified to channel the coolant through the base plate without tubes, freeing up space for the microprocessor boards. The coolant was instead channeled through banjo bolts which fastened the cooling plates to the individual modules (Figure 5.2). The cold plates were also modified to allow ribbon cables to connect the module board to the microprocessor board. Copper strips were used to connect each of the modules together in series. This third generation TEG was designed to be the first generator to measure total power generation in series, rather than adding up the power produced by individual modules wired in parallel. 


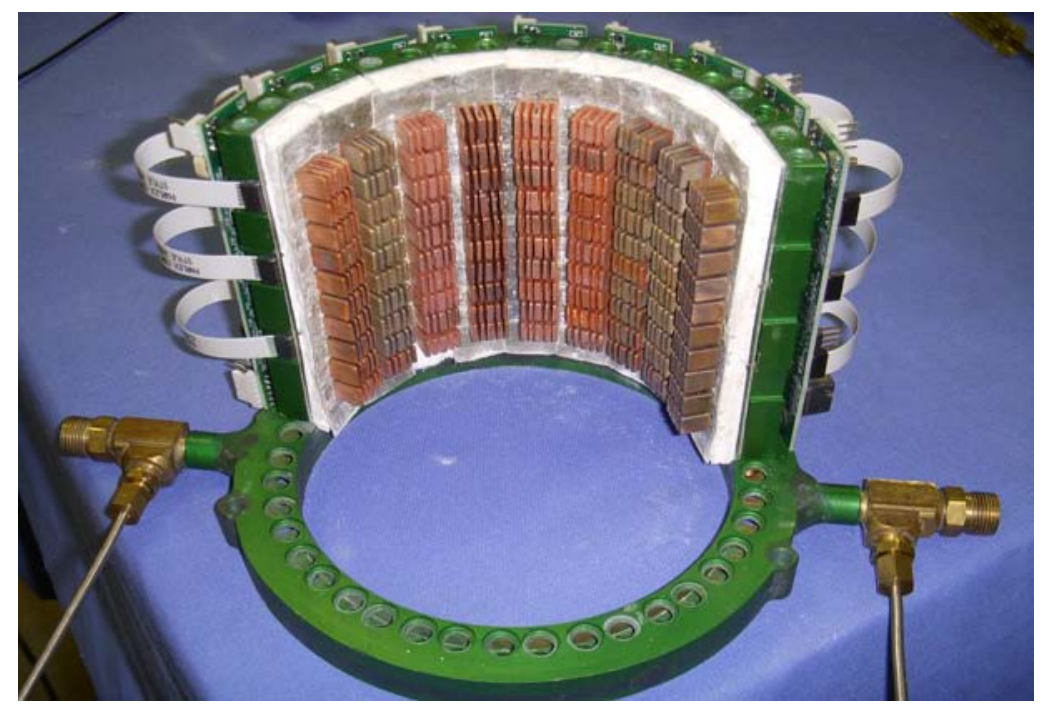

Figure 5.2. Partially assembled thermoelectric generator (generation 3).

Preliminary testing was conducted using two $5 \mathrm{~W}$ modules in the generator. Not a single couple broke during these tests even after being cycled twice to hot side temperatures of $565^{\circ} \mathrm{C}$ and $700^{\circ} \mathrm{C}$. This success prompted expectations of similar results for the next test of $100 \mathrm{~W}$. We set up the test using the same round generator housing design, leg size and material, and layering as with the two five watt test. We added a microcontroller board to control the EASs on our module board to bypass broken couples. Finally, we insulated the couples using aerogel with a mica sheet on top. We also wired every module in series to reach higher voltages of roughly 15 volts at max load. The load was a bank of incandescent light bulbs. Figure $\mathbf{5 . 3}$ shows pictures of the generator setup that was tested.
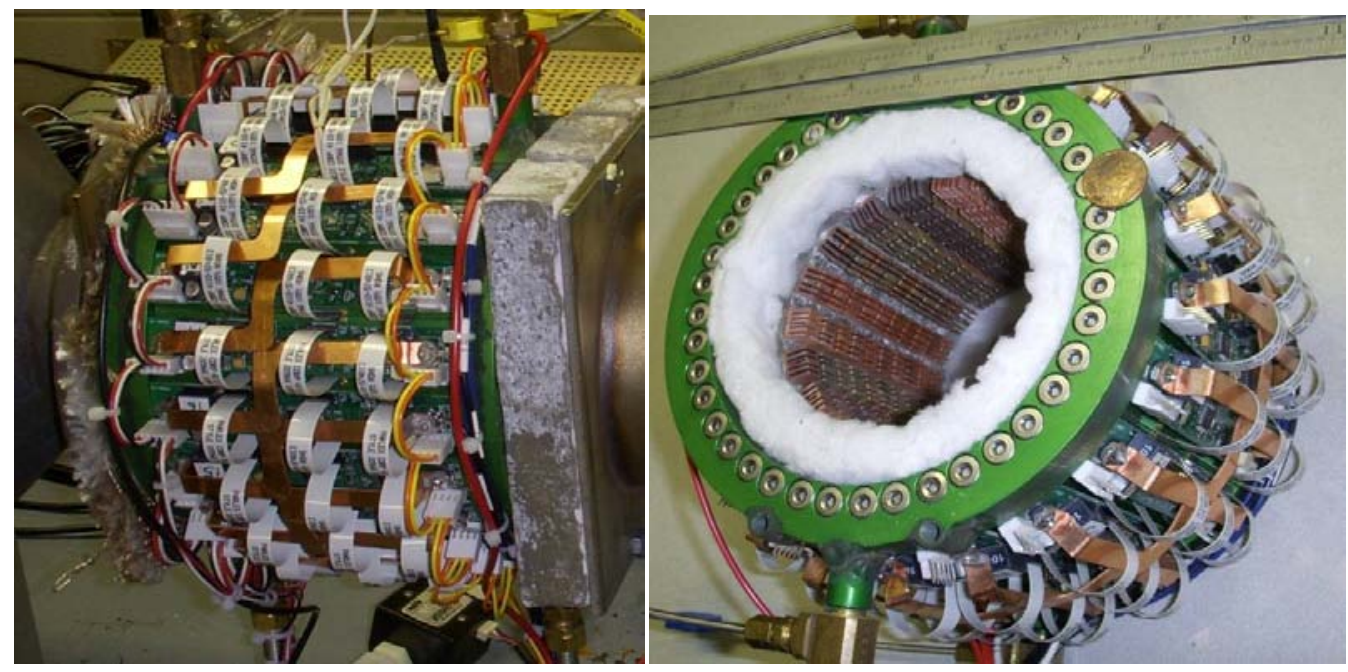

Figure 5.3. Generator mounted on the air torch bench (left). Generator totally assemble and ready to be mounted onto the air torch (right). 
Once the hot side of the couples reached roughly 460 degrees the couples started to fail on a mass scale. Out of 200 couples in the generator 156 of them broke (78\%). Out of the 156 broken couples, 155 of them had broken P-legs. This was quite unexpected, considering that we used the same leg parameters in this generator as we did in previous test that had produced great results. The only major variation made in this test was the insulation. In the attempt to make the generator perform better by insulating it with aerogel, the aerogel had an adverse effect on the Pleg, creating a condition which caused it to break. Figure 5.4 shows the voltages of 5 different modules, the hot side temperature and thAT across the c ouples. As the hot side temperature reaches 460 Celsius, the voltages begin to decline, showing a higher resistance change and eventually leading to a break in the couple. The spikes in voltage indicate when the couple had failed enough for the microcontroller to activate the EAS and short out the broken couple.

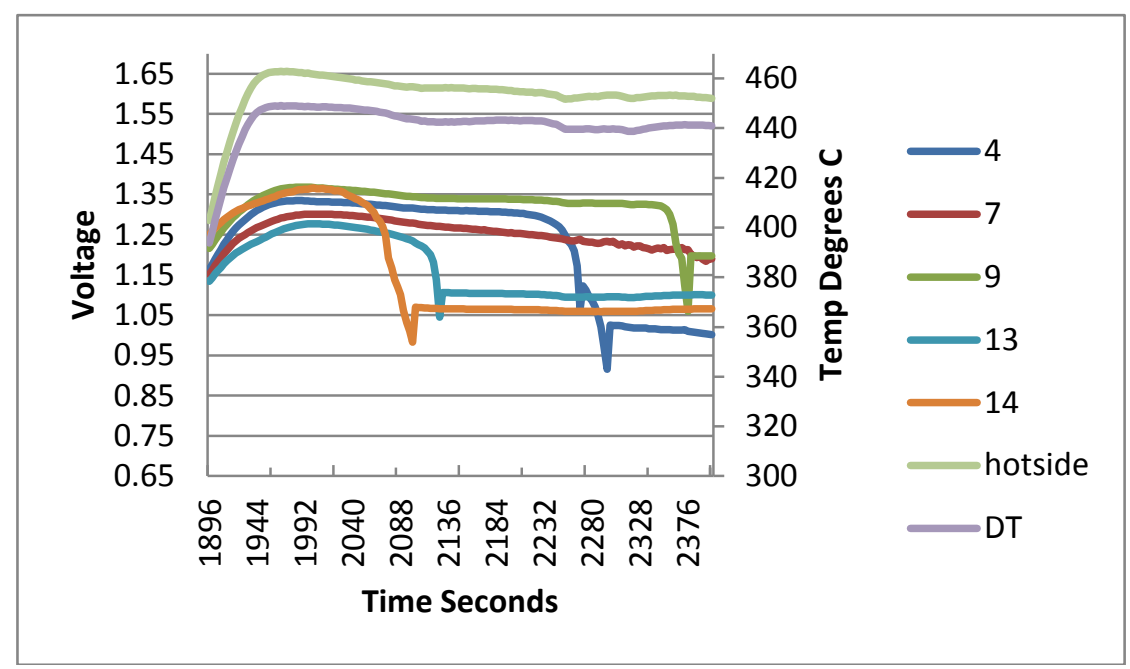

Figure 5.4. Voltages of 5 different modules, the hot side temperature and the $\Delta \mathrm{T}$ across the couples.

To prove our hypothesis (the insulation creates a condition that leads to P leg failures) we conducted another test on 3 separate 5 watt modules, changing only the insulation.

\subsubsection{Watt Insulation Test}

While the $100 \mathrm{~W}$ airtorch test resulted in catastrophic failure of the P-leg, the single-couple cycling tests performed with the same material had 100\% success rates, despite the higher hot side temperatures (up to $650^{\circ} \mathrm{C}$ ). It is believed that the consistently high success rates of the cycling tests are due to compression of the couple while cycling. Even a similar $15 \mathrm{~W}$ airtorch test (Hot side temp of $550^{\circ} \mathrm{C}$ ) produced a $100 \%$ success rate. To investigate the effect of insulation types on the success rates of the thermoelectric couples, another airtorch test was conducted, featuring three types of insulation: aerogel with Mica (the insulation type used in the $100 \mathrm{~W}$ airtorch test), aerogel only, and ceramic wool (Figure 5.5). 


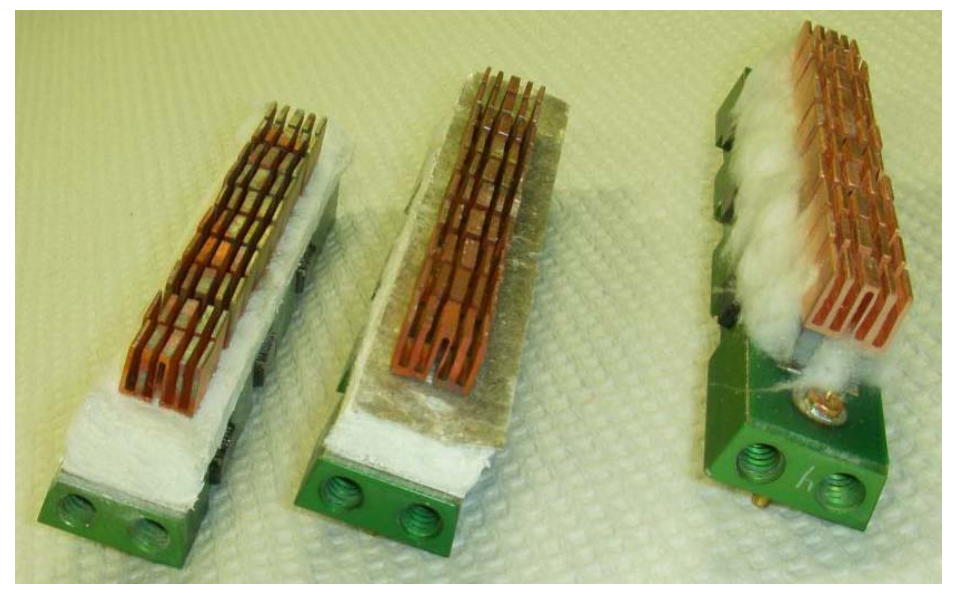

Figure 5.5. Left to right: aerogel only, aerogel with mica, and wool.

All of the skutterudite material used in this test came from the same N-type and P-type pucks. Both of the pucks were fabricated and pressed to most closely resemble the material used in the $100 \mathrm{~W}$ airtorch test. The bonding process and the module assembly process were also performed in the same manner as the previous test. The three modules being tested were spaced in the generator such that one "dummy" module was placed between two test modules in the following order: wool, dummy module, aerogel with Mica, dummy module, and aerogel only. The microprocessor boards were connected for the three test modules; the 17 modules that were not being tested were not connected to the microprocessor boards, power, or load.

One significant deviation from the previous test was the way in which power was measured. During the $100 \mathrm{~W}$ airtorch test, as well as most of the tests before it, the modules were back loaded with a Kepco power supply to measure the generated power. For this test, power was measured by loading each individual module with a 3 level resistor bank: .77 ohms, .44 ohms and $.11 \mathrm{ohms.}$ Power was then calculated using the following relation:

$\mathrm{P}=\mathrm{V}^{2} / \mathrm{R}$

Figure 5.6 shows the graphs of the voltage of each module as a function of time. For the beginning portion of the test, the voltages for all three modules rose as the airtorch heated the copper heat exchangers to a temperature of approximately $420-450^{\circ} \mathrm{C}$. At approximately 1,600 seconds the voltage of the two modules with aerogel insulation peaked and started to decline. This is due to an increase in electrical resistivity in one or more of the thermoelectric legs, an indication of failing structural integrity. As programmed in the CBT software, once a couple reaches a certain negative voltage for a specified amount of time, the corresponding EAS is activated and electrically bypasses the failed couple. A sharp jump in voltage is seen as the current is no longer forced to pass through the high-resistance couple. As is seen in Figure 5.6, 7 couples in the module with aerogel insulation broke during this portion of the test; 8 couples broke in the module with aerogel and mica. Only one couple broke during the entire test with wool insulation. 
One possible explanation for this discrepancy is the difference in hot side temperatures between the modules. Figure 5.7 shows both of the modules insulated with aerogel attained a hot side temperature of over $530^{\circ} \mathrm{C}$, while the hot side temperature of the module with wool insulation peaked at approximately $480^{\circ} \mathrm{C}$. While aerogel is clearly the better insulator, the higher temperatures increase the thermal stresses at the hot side interface, where all of the breakages occurred. Further analysis is ongoing to identify and mitigate the failure modes at the hot side interface.

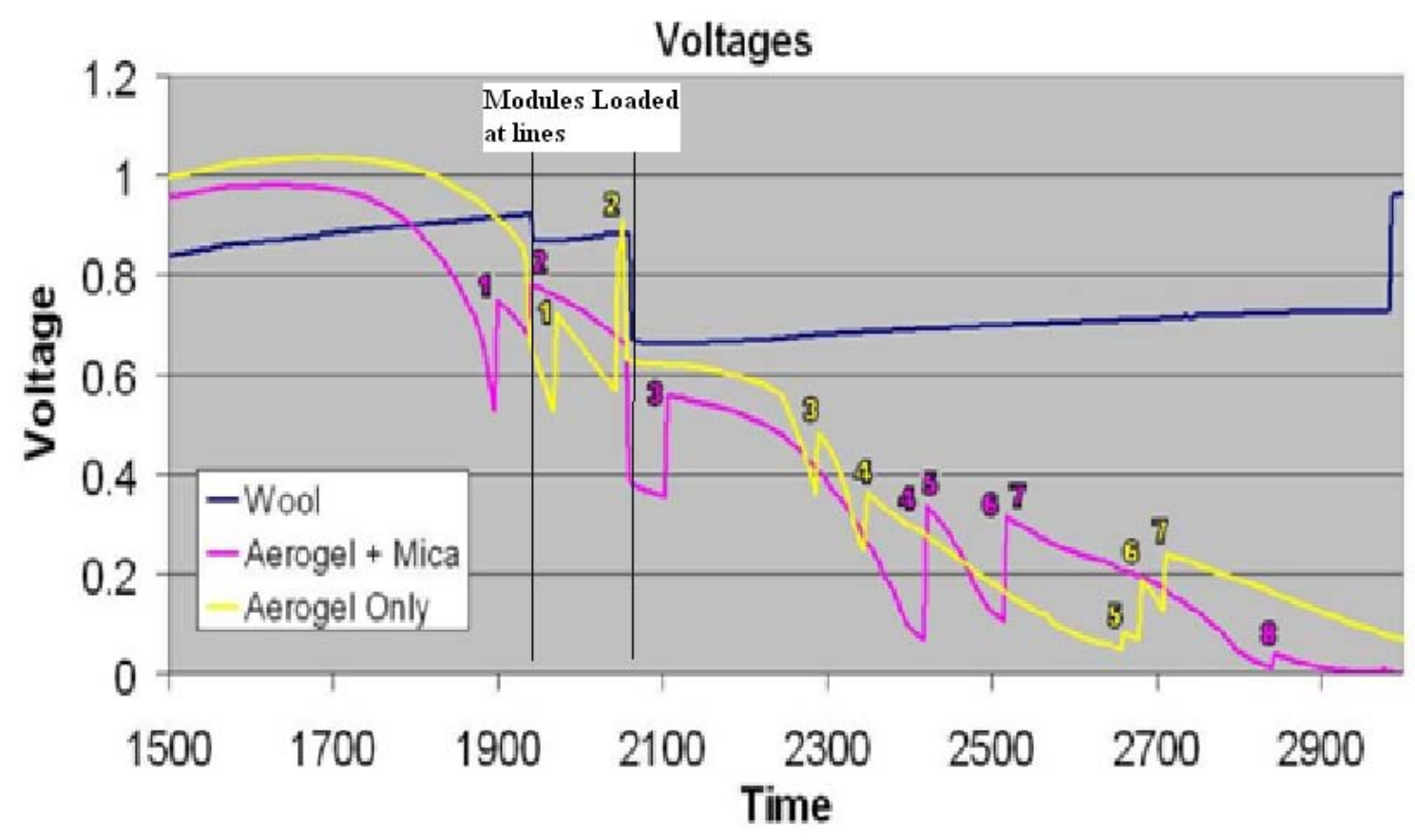

Figure 5.6. Voltages of the 3 modules tested and the couple breakage history. 


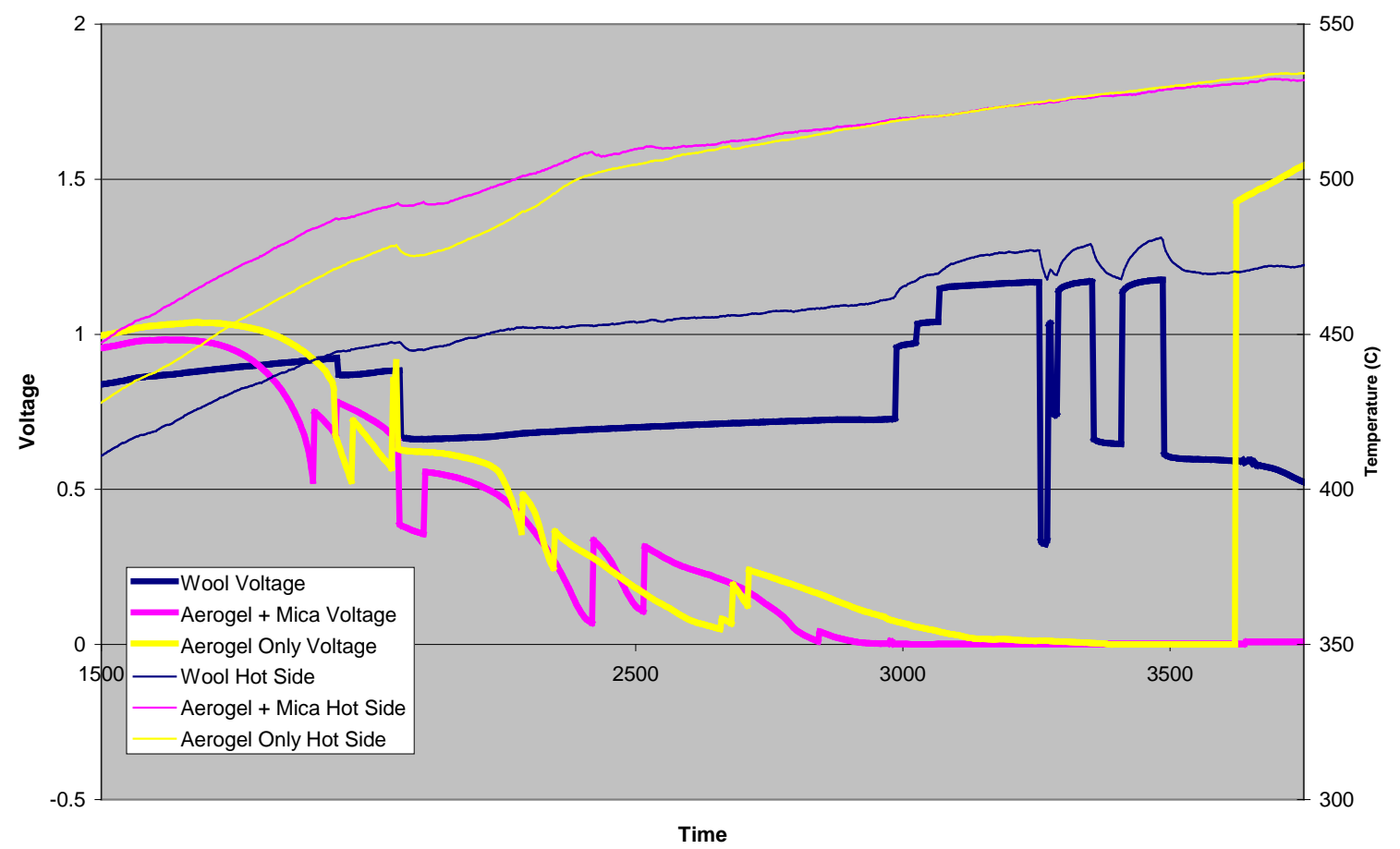

Figure 5.7. Module voltages with the hot side temperature and the $\Delta \mathrm{T}$ across the couples.

\section{TEG Insulation}

\subsection{Aerogel Activities}

We have continued to work on efforts to improve thermal insulation efficacy, characterizing the stability of skutterudite at high temperatures in air using aerogel as a protective coating and to further pursue the ability to ambiently dry aerogel. Thermal insulation efficacy will be conducted using pseudo-calorimetric techniques as well as infrared imaging analysis.

Efforts to improve the aerogel casting process focused on a new integration approach. Instead of casting the aerogel around the TE subassemblies, we are exploring methods that cast the aerogel into molds that mimic the TE subassemblies, such that the aerogel can be inserted into place as dry, robust pre-forms. This approach could facilitate mass production efforts; the pre-formed aerogel pieces are easier to handle and process compared to the cast-in-place process. We will need to evaluate the effectiveness of these preferred insulations compared to those that are cast in place.

The pre-formed approach was modified to adjust for the new, smaller cross-section thermoelectric couple design. The tighter dimensional constraints proved challenging for the aerogel. Some shrinkage and cracking occurred during supercritical drying. A new reinforcement scheme is being developed and should minimize shrinkage and improve durability. This involves silica fibers of different length scales - from short fibers $(\sim 100 \mu \mathrm{m}$ in 
length) to a felt mat (spanning the entire width and length of the sample). An additional modification using mica sheets to sandwich the insulation provides an additional barrier to gas convection, guards against possible gaps between adjacent modules, and improves handling of the final product. Pictures of a finished aerogel sample along with the casting mold can be seen in Figure 6.1.

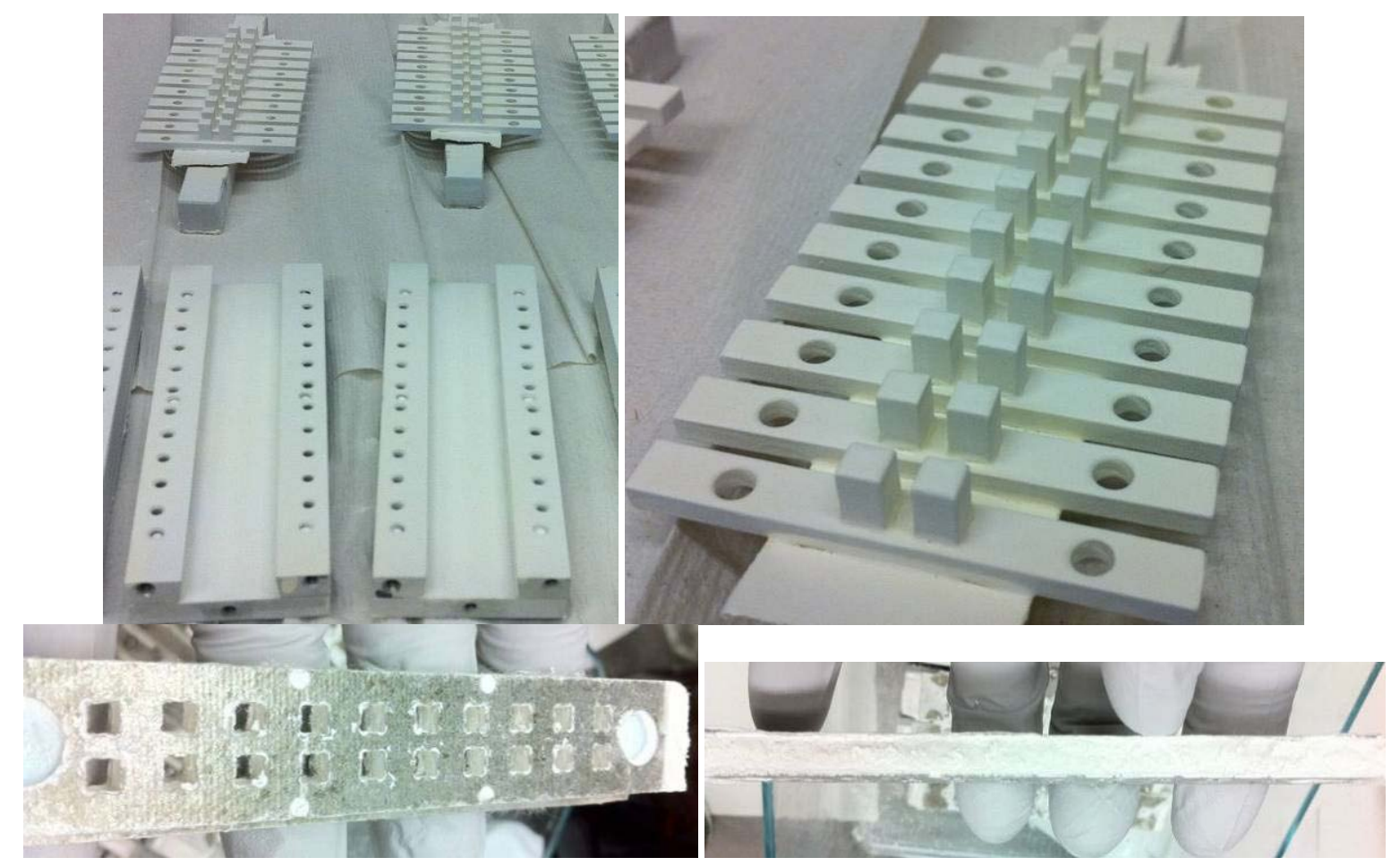

Figure 6.1. The aluminum molds mimic the subassembly features (top left).

Close up of aluminum mold insert (top right).

Dried aerogel insulation with mica sheets (bottom).

\section{Couple Bypass Technology (CBT)}

\subsection{Background}

The 100 Watt thermoelectric generator is made up of twenty thermoelectric modules connected in series. Each thermoelectric module has 10 thermoelectric couples connected in series for a total of two hundred series connected couples or devices (TEDs).

In this application, the thermoelectric devices are used to convert thermal energy to electrical energy. A temperature gradient is formed across each device and generates a voltage potential. The thermoelectric generator can be considered as a voltage source connected with source impedance (see Figure 7.1). Due to the source impedance, a resistant load can be varied to maximize the power output. The source impedance is considered as the resistive impedance. 


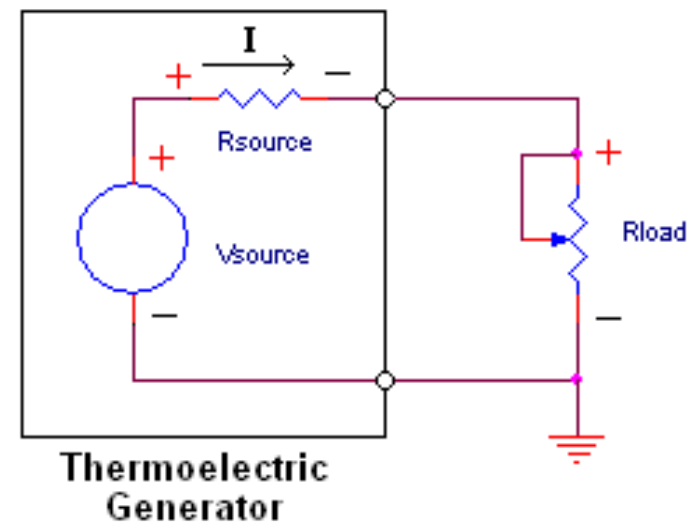

Figure 7.1. Electric circuit of a TE device.

Although the thermoelectric generator can be macroscopically viewed as a single voltage source, it is actually the summation of many individual voltage sources (Figure 7.2). For the 100 Watt generator, the total number of voltage sources is 200 .

The no-load (open circuit) output voltage of the generator is

$$
\text { Vout }_{\text {No Load }}=\sum_{i=1}^{n} V_{i}
$$

When a load resistance, $\mathrm{R}$ load, is connected to the output of the generator, the output voltage is

$$
\text { Vout }=\frac{R_{\text {Load }}}{\sum_{i=1}^{n} R_{i}+R_{\text {Load }}} \sum_{i=1}^{n} V_{i}
$$

where $R_{i}$ and $V_{i}$ are the source resistance and voltage of the $\mathbf{i}$-th TE device. 


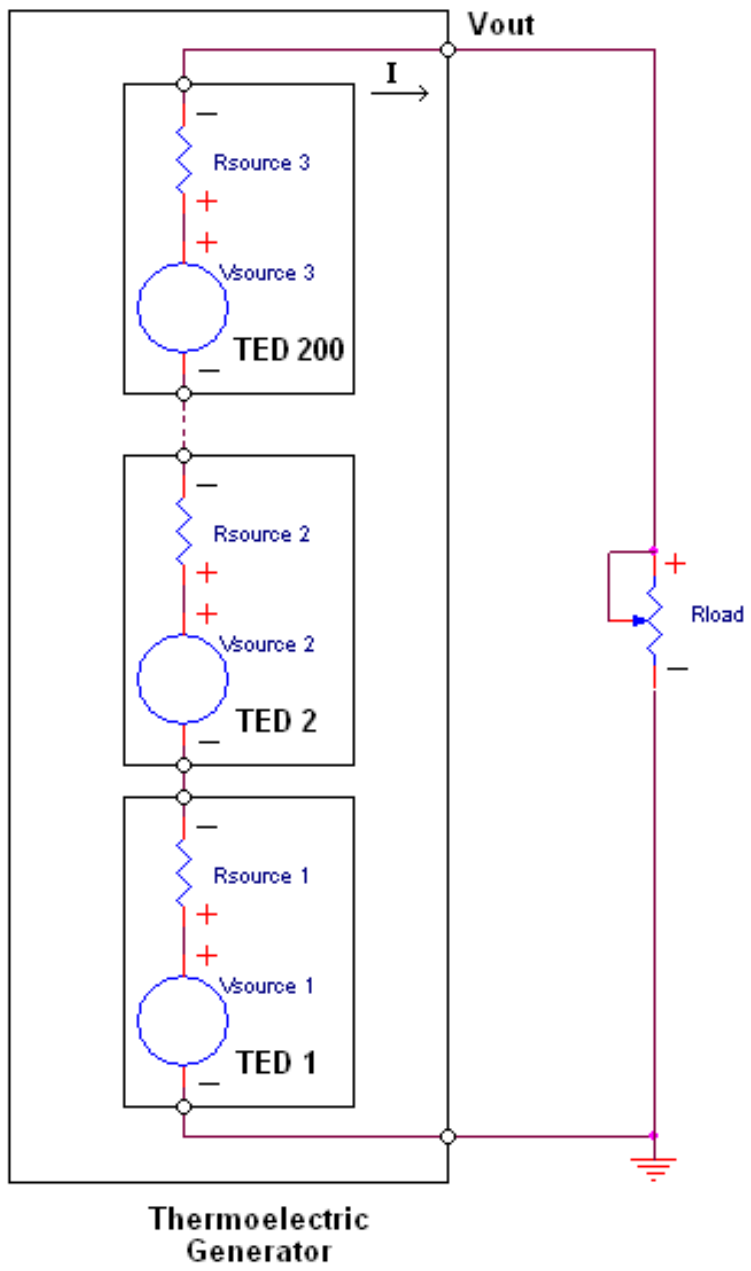

Figure 7.2. Electric circuit of a TE generator.

\subsection{Shorting Switches Application}

The prevalent failure mode of the thermoelectric devices is open circuit. Since these devices are in series, a failure of any one device would disable the entire generator. Therefore, an electrically activated switch (EAS) is placed across each device to enable bypassing that device in the event of a failure (Figure 7.3). These switches are intended to be active only when a failure is present. However, it takes power to activate an EAS, which must be supplied by the other couples in the series. Thus, if one couple fails, the power produced by another couple is needed to power the EAS that bypasses the failed couple. 


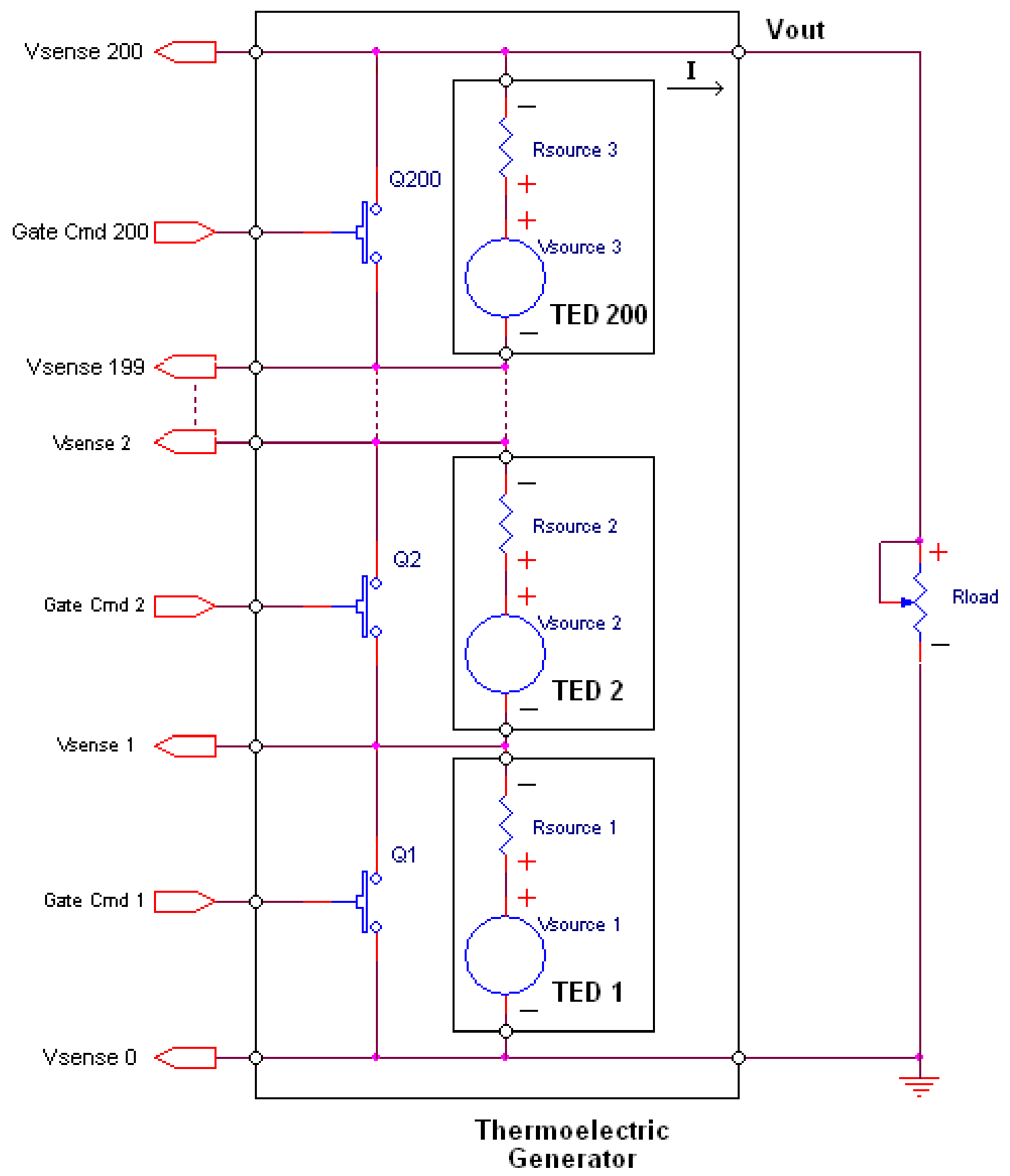

Figure 7.3. Control circuit of a TE generator.

As stated above, when one of the EASs is activated it consumes power. This is a negative effect of using these devices, considering the main goal of our TED generator is to generate power. The power that is consumed by the EAS is proportional to the amount of current that is put through the device. At the peak performance of the couples, an EAS consumes approximately 0.5 Watts if it is activated. The power curve of an EAS can be seen in Figure 7.4. When a couple or a series of couples fails in a module the breakeven point is 5 couples breaking. If any more than 5 
couples break in a module it no longer will produce power for the generator; it will be taking the power away. This is because if a module breaks you lose the power it produced (approximately .5 Watts). You lose the power that the EAS consumes which keeps the electrical circuit closed. (Figure 7.4)
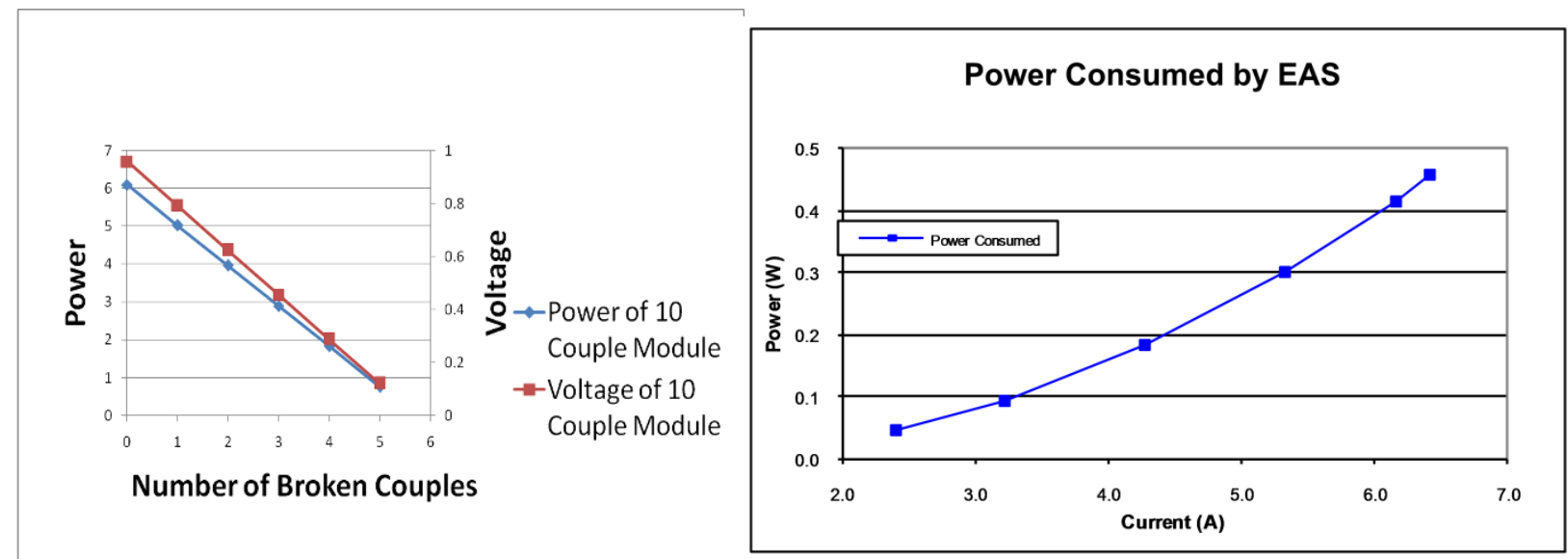

Figure 7.4. Graph of the number of couples that are broken in a module and the power/voltage of the module (left).

The power curve of an EAS (right).

\subsection{Shorting Circuit Architecture}

The 100 Watt thermoelectric generator is partitioned into 20 modules. Each module contains 10 thermoelectric devices and each module has two circuit boards. One circuit board is mounted on the inside of the cold shoe and the other circuit board is mounted on the outside. The inside circuit board (lower board shown in Figure 7.5) is populated with the 10 thermoelectric devices providing the electrical conductivity for both legs of each TED. Additionally, the inside board is populated with 10 shorting switches. The outside circuit board (upper board shown in Figure 7.5) is populated with the microcontroller and supporting circuitry that controls the shorting switches. The outside board also has a CAN transceiver for communicating with an optional supervisory controller or data acquisition system. The system architecture is illustrated in Figure 7.6. 


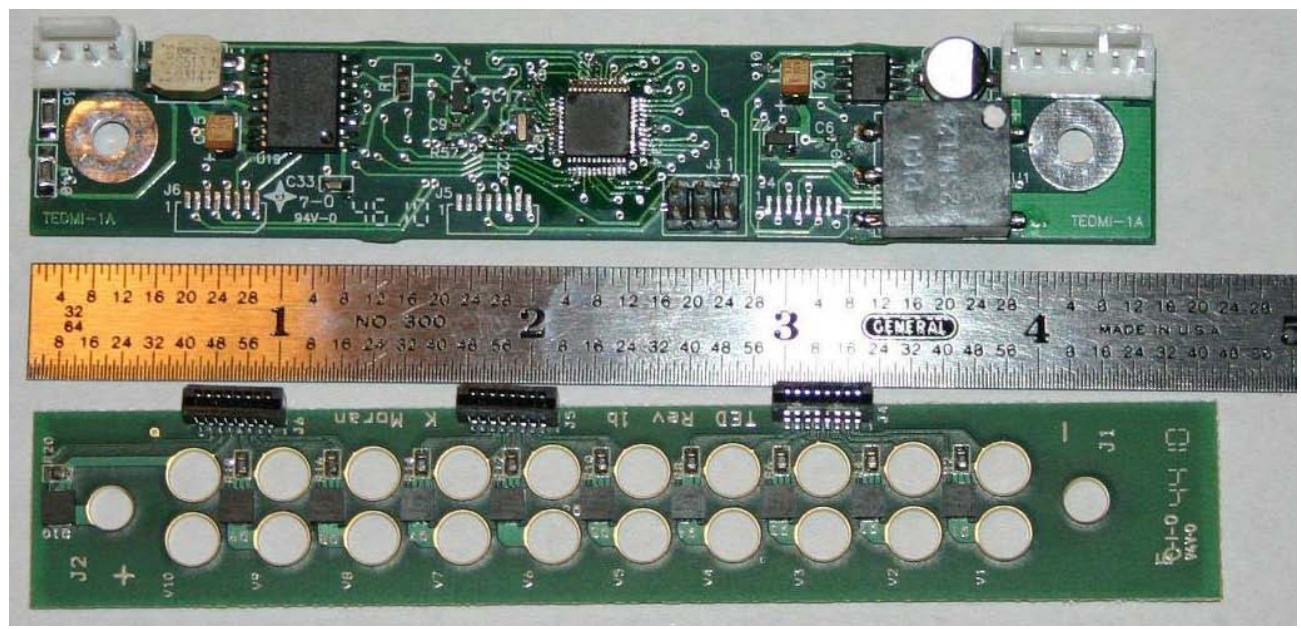

Figure 7.5. Control circuit boards.

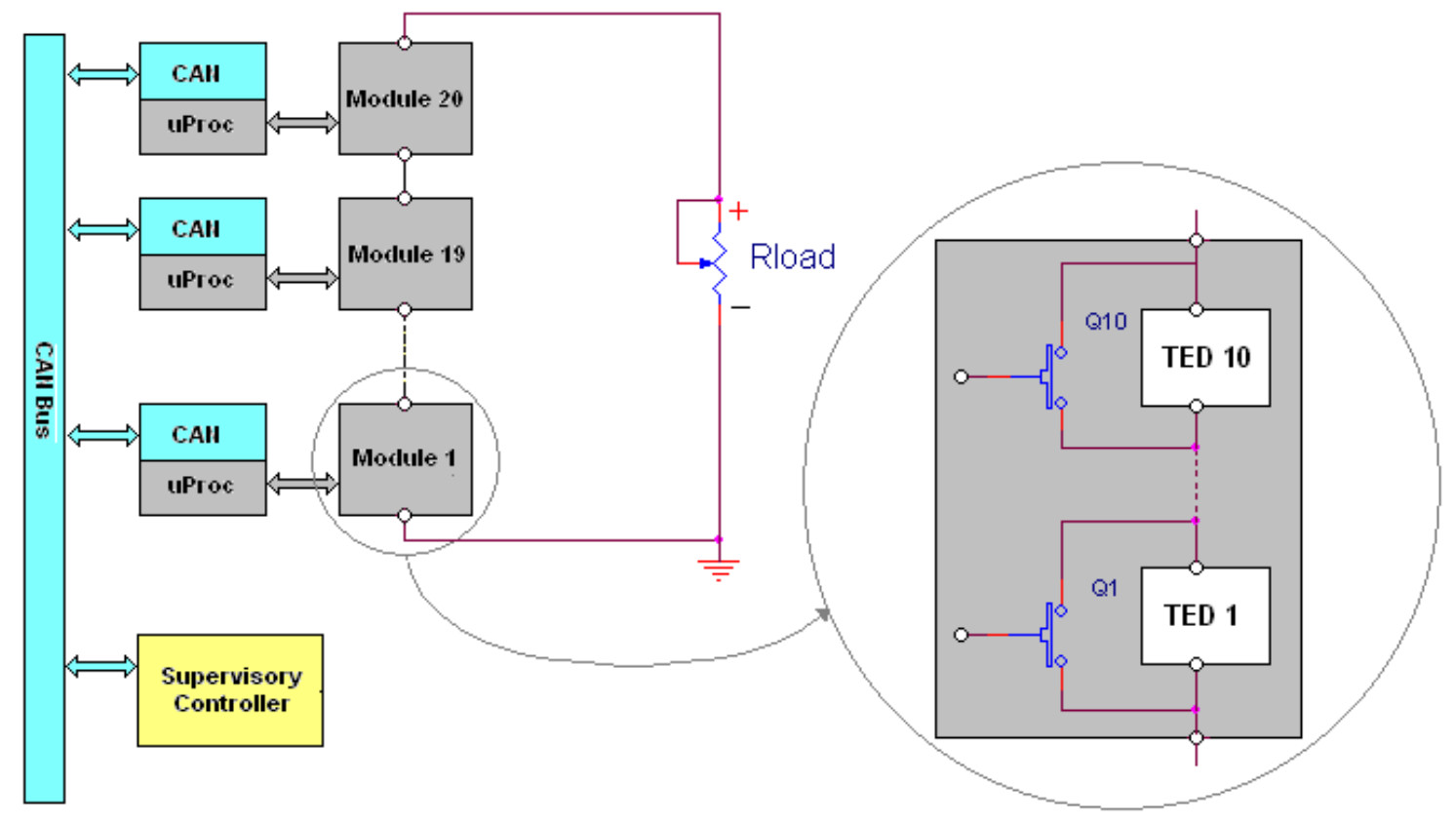

Figure 7.6. Control system architecture. 
Figure 7.7 shows a partially assembled generator with fully assembled cold shoes including both circuit boards.

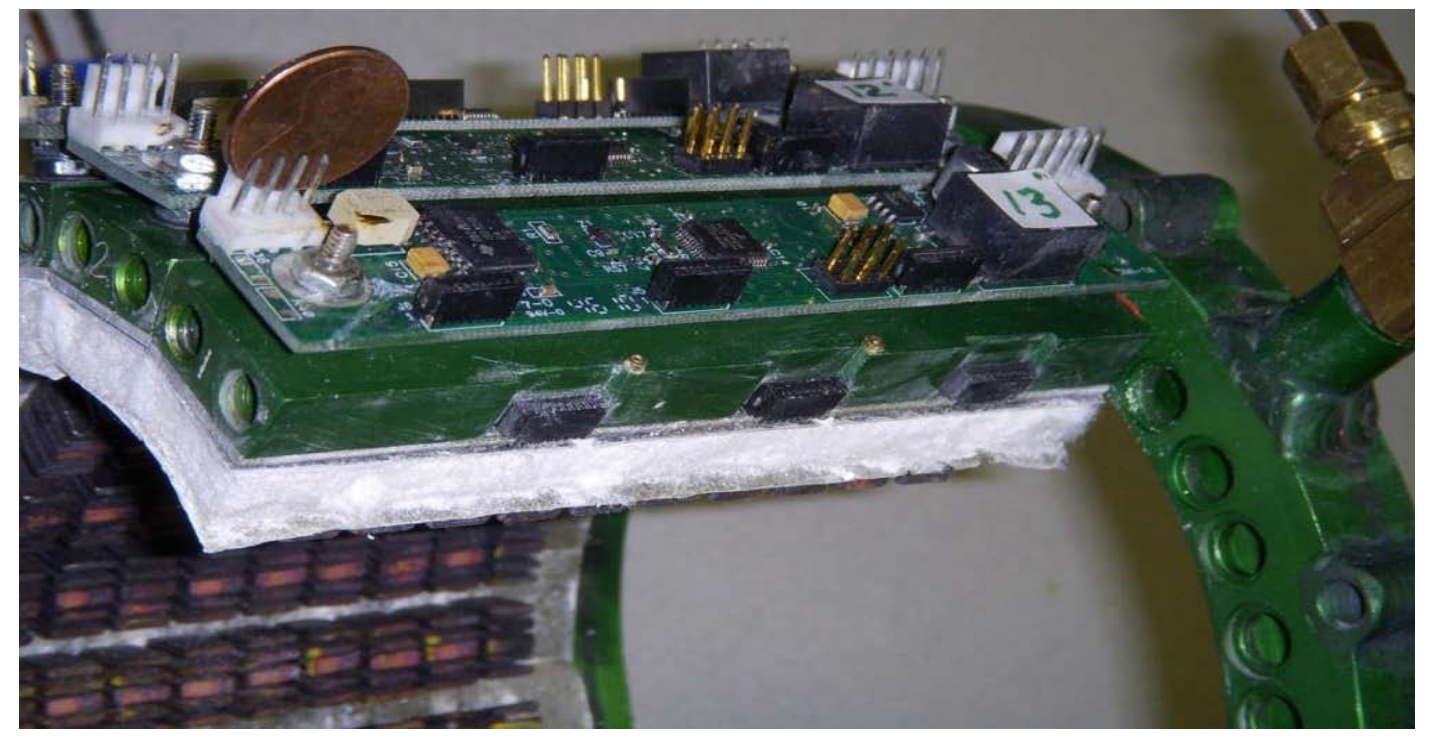

Figure 7.7. TEG module and parts.

Figure 7.8 (left) shows a parts break-down of the cold shoe, inner circuit board, and thermoelectric elements, and the right side shows the inner circuit board assembled and mounted on the cold shoe.
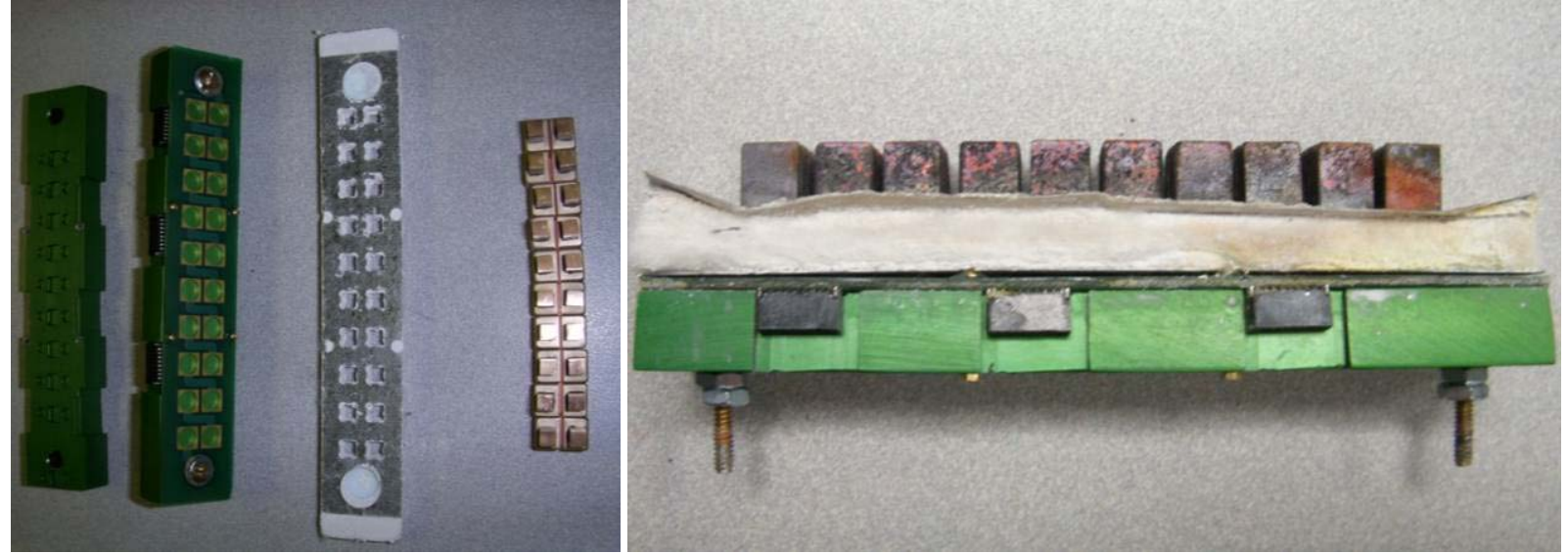

Figure 7.8. Partially assembled TE. 
The inner and outer circuit boards are connected by 3 ribbon cables. Solid copper bands connect the modules in series. Figure 7.9 shows the assembled generator with ribbon cables and series connections.

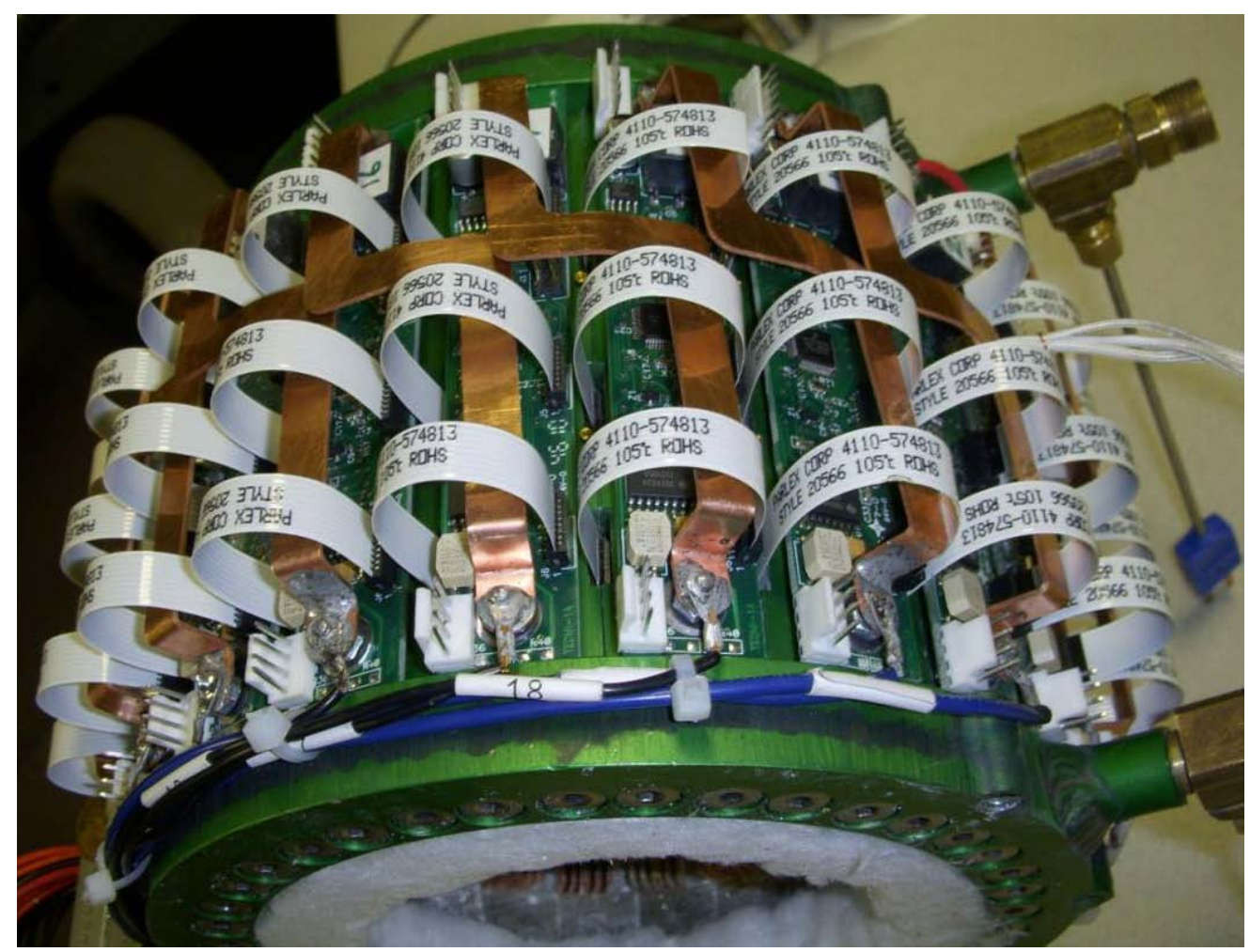

Figure 7.9. Assembled TE generator.

\subsection{Summary}

The thermoelectric generator shorting system provides the capability to monitor and short-out individual thermoelectric couples in the event of failure. This makes the series configured thermoelectric generator robust to individual thermoelectric couple failure. Open circuit detection of the thermoelectric couples and the associated short control is a key technique to ensure normal functionality of the TE generator under failure of individual TE couples.

This report describes a five-year effort whose goal was the understanding the issues related to the development of a thermoelectric energy recovery device for a Class-8 truck. Likely materials and important issues related to the utility of this generator were identified. Several prototype generators were constructed and demonstrated. The generators developed demonstrated several new concepts including advanced insulation, couple bypass technology and the first implementation of skutterudite thermoelectric material in a generator design. Additional work will be required to bring this system to fruition. However, such generators offer the possibility of converting energy that is otherwise wasted to useful electric power. Uur studies indicate that this can be accomplished in a cost-effective manner for this application. 


\subsection{Acknowledgements}

This nearly six-year effort was in no small part made possible through the continuous support of the DOE under contract DE-FC26-04NT 42281. Samuel Taylor, NETL (DOE Project Officer) deserves special recognition for his technical advice and administrative support. We are also grateful to John Fairbanks (DOE Technology Development Manager), for his tireless efforts on behalf of the Thermoelectrics Community with the goal of bringing this technology to a position where it can be utilized for improving the energy independence of the US by converting waste heat into useful electricity in transportation systems.

\section{References}

1. G. L. Bennett, "Space Applications," in CRC Handbook of Thermoelectrics, ed. D. M. Rowe, CRC Press LLC, Boca Raton, FL, 1995.

2. H. J. Goldsmid, R. W. Douglas, "The Use of Semiconductors in Thermoelectric Refrigeration,” British Journal of Applied Physics, 5, 386-390, 1954.

3. Hicks,L.D.; Dresselhaus, M.S.; "Effect of Quantum-Well Structures on the Thermoelectric Figure of Merit,” Physical Review B 47 (19) 12727-12731, 1993.

4. M.C. Algrain, "Electrification of On-Highway Trucks," MIT Industry Consortium on Advanced Automotive Electrical/Electronic Components and Systems, Program Review Meeting, Shelter Pointe Hotel, San Diego, CA. 27-28 April, 2004.

5. Frank P. Incropera and David P. Dewitt, Introduction to Heat Transfer, 2002, $4^{\text {th }}$ ed., John Wiley \& Sons, Inc.

6. Robert W. Fox, Alan T. McDonald, Introduction to Fluid Mechanics 3rd Edition; John Wiley \& Sons Inc.; New York, NY, 1985; p 366-368.

7. Yunus A. Cengel, Heat Transfer, A Practical Approach 2nd Ed; McGraw-Hill; New York, NY, 2003; p 11.

8. Yunus A. Cengel, Heat Transfer, A Practical Approach 2nd Ed; McGraw-Hill; New York, NY, 2003; p 427.

9. http://www.engineeringtoolbox.com/nitrogen-d_977.html; accessed August 6, 2009

10. http://www.engineeringtoolbox.com/water-thermal-properties-d_162.html; accessed August 6, 2009.

11. B.D. Hall. "Powder Processing, Powder Characterization, and Mechanical Properties of LAST (Lead-Antimony-Silver-Tellurium) and LASTT (Lead-Antimony-Silver-Tellurium Tin) Thermoelectric Materials,” Unpublished Master's Thesis, Michigan State University, 2008.

12. R.W. Rice. “Porosity of Ceramics,” Marcel Dekker, New York (1998) 43-167.

13. R.D. Schmidt, J.E. Ni, E.D. Case, J.S. Sakamoto, D.C. Kleinow, B.L. Wing, R.C. Stewart, E. J. Timm. "Room temperature Young's Modulus, Shear Modulus, and Poisson's Ratio of Ce0.9Fe3.5Co0.5Sb12 and Co0.95Pd0.05Te0.05Sb3 Skutterudite Materials,” J. Alloys Compd. (2010) 303-309.

14. F. Ren, E.D. Case, J.E. Ni, E.J. Timm, E. Lara-Curzio, R.M. Trejo, C.-H. Lin, M.G. Kanatzidis. "Temperature-Dependent Elastic Moduli of Lead Telluride-Based Thermoelectric Materials,” Philos. Mag. 89 (2009) 143-167.

15. J.B. Wachtman, “Mechanical Properties of Ceramics,” Wiley, New York (1996) 92-115. 


\section{Appendix A. Journal papers, proceedings papers and technical presentations funded either in part or entirely by DOE}

- Harold Schock et al., "Prospects for Implementation of Thermoelectric Generators as Waste Heat Recovery Systems in Class 8 Truck Applications,” in preparation.

- Robert Schmidt, Jennifer E. Ni, Eldon D. Case, Jeffery Sakamoto, Daniel Kleinow, Bradley Wing, Ryan Stewart, Edward J. Timm, "Room Temperature Young’s Modulus, Shear Modulus, Poisson's Ratio and Hardness of $\mathrm{Ce}_{0.9} \mathrm{Fe}_{3.5} \mathrm{Co}_{0.5} \mathrm{Sb}_{12}$ and $\mathrm{Co}_{0.95} \mathrm{Pd}_{0.05} \mathrm{Te}_{0.05} \mathrm{Sb}_{3}$ Skutterudite Materials," submitted, Journal of Alloys and Compounds

- Hu, K., Chi, X., Shih, T.I-P., and Schock, H.J., "Heat Transfer in Thermoelectric-PowerGeneration Devices," to be submitted to the AIAA J. of Thermophysics.

- Hu, K., Chi, X., Shih, T.I-P., and Schock, H.J., (Jan. 2009) "Heat Transfer Enhancement in Thermoelectric Power Generation," AIAA 2009-1210.

- Cao, D., and Peng, F. Z., (2009) "Zero-Current-Switching Multilevel Modular SwitchedCapacitor DC-DC Converter," IEEE Energy Conversion Congress and Exposition, ECCE 2009. Digest Submitted.

- Cao, D., and Peng, F. Z., (2009) "A Family of Z-Source and Quasi-Z-Source DC-DC Converters," Applied Power Electronics Conference and Exposition, APEC Twenty-Fourth Annual IEEE, Feb. 2009.

- Ren, F; Case, ED; Ni, JE, et al. (2009) "Temperature-Dependent Elastic Moduli of Lead Telluride-Based Thermoelectric Materials,” PHILOSOPHICAL MAGAZINE, Volume: 89, Issue: 2, Pages: 143-167.

- Hall, B. D., Case. E. D., Ren, F., Johnson, J., and Timm, E.J., (2009) “Agglomeration During Wet Milling of LAST (Lead-Antimony-Silver-Tellurium) Powders," Materials Chemistry and Physics, 113[1]: $497-502$.

- Ren, F., Case, E. D., Ni, J. E., Timm, E. J., Lara-Curzio, E., Trejo, R. M., Lin, C.-H., Kanatzidis, M. G., (2009) “The Young’s Modulus and Poisson's Ratio of Lead-Telluride Based Thermoelectric Materials as a Function of Temperature,” Philosophical Magazine, 89[2]: 143167.

- Ren, F., Case, E. D., Hall, B. D., Ni, J. E., Timm, E. J., Wu, C-I., Hogan, T., and LaraCurzio, E., (2008) "Mechanical Properties of Slow-Cooled Ingot LAST (Pb-Sb-Ag-Te) Thermoelectric Materials at Room Temperature,” accepted for publication, Philosophical Magazine Letters.

- Han, MK; Kong, HJ; Uher, C, et al. (2008) "Investigation of Thermoelectric Materials: Substitution Effect of Bi on the Ag1-xPb18MTe20 (M=Sb, Bi) (x=0,0.14, 0.3)," Conference 
Information: 8th Symposium on Thermoelectric Power Generation, held 2007 MRS Fall Meeting, Date: NOV 26-29, 2007 Boston MA, Source: Thermoelectric Power Generation, Volume: 1044, Pages: 95-100.

- Ren, F., Case, E. D., Timm, E. J., Lara-Curzio, E., and Trejo, R. M., "Temperature Dependent Thermal Expansion of Cast and Hot Pressed LAST (Pb-Sb-Ag-Te) Thermoelectric Materials," submitted 2008, Philosophical Magazine.

- Ren, F., Hall, B. D., Ni, J. E., Case, E. D., Timm, E.J., Schock, H. J., Wu, C-I., D’Angelo, J. J., Hogan, T. P., Trejo, R. M., Lara-Curzio, E., (2008) "Mechanical Characterization of PbTeBased Thermoelectric Materials,” Thermoelectric Power Generation, Materials Research Society Proceedings, Vol. 1044. p 121 - 126, Warrendale, PA: Materials Research Society.

- D’Angelo, J. J., Timm, E. J., Ren, F., Hall, B. D., Case, E., Schock, H., Kanatzidis, M., Chun, D.-Y., Hogan, T. P., (2008) "Electrical Contact Fabrication and Measurements of Metals and Alloys to Thermoelectric Materials," Materials Research Society Symposia Proceedings, vol. 1044, pp. 449-455.

- Ni, Jennifer E., Ren, Fei, Case, Eldon D., Timm, Edward J., (2008) "Porosity Dependence of Elastic Moduli in LAST (Lead-antimony-silver-tellurium) Thermoelectric Materials," submitted, Materials Chemistry and Physics.

- Chen, L., Cao, D., Huang, Y., and Peng, F. Z., (2008) "Modeling and Power Conditioning for Thermoelectric Generation," in Power Electronics Specialists Conference, June 2008. PESC, IEEE, pp. 1098-1103.

- Ren, F., Case, E. D., Sootsman, J. R., and Kanatzidis, M. G., Kong, H., Uher, C., LaraCurzio, E., and Trejo, R. M., (2008) "The High Temperature Elastic Moduli of Polycrystalline PbTe Measured by Resonant Ultrasound Spectroscopy,” ACTA MATERIALIA, Volume: 56, Issue: 20, Pages: 5954-5963.

- Wu, C.-I., Timm, E. J., Ren, F., Hall, B. D., Ni, J., Downey, A., D’Angelo, J., Short, J., Schock, H., Case, E., Sootsman, J., Han, M.-K., Kanatzidis, M., Chun, D.-Y., Hogan, T. P., (2008) "Study on the Fabrication and Characterization of LAST and LASTTT Based Thermoelectric Generators,” Materials Research Society Symposia Proceedings, vol. 1044, pp. 441-447.

- Sootsman, J; Kong, H; Uher, C, et al. (2008) "Transport Behavior and Thermal Conductivity Reduction in the Composite System PbTe-Pb-Sb," Conference Information: 8th Symposium on Thermoelectric Power Generation held 2007 MRS Fall Meeting, Date: NOV 26-29, 2007 Boston MA, Source: THERMOELECTRIC POWER GENERATION, Volume: 1044, Pages: 327-332.

- Gueguen, A; Poudeu, PFP; Pcionek, R, et al (2008) "Thermoelectric Properties of the Nanostructured NaPb18-xSnxMTe20 (M=Sb, Bi) Materials,” Conference Information: 8th 
Symposium on Thermoelectric Power Generation held 2007 MRS Fall Meeting, Date: NOV 2629, 2007 Boston MA, Source: THERMOELECTRIC POWER GENERATION, Volume: 1044, Pages: 349-354.

- $\quad$ Ren, F., Case, E. D., Hall, B. D., Ni, J. E., Timm, E. J., Wu, C-I., Hogan, T., and LaraCurzio, E., "Mechanical Properties of Slow-Cooled Ingot LAST (Pb-Sb-Ag-Te) Thermoelectric Materials at Room Temperature,” accepted for publication, Philosophical Magazine Letters.

- $\quad$ Ren, F., Case, E.D., Hall, B.D., Timm, E.J., and Schock, H.J., (2007). "Young's Modulus of N-Type Last Thermoelectric Material Determined from Knoop Indention,” Chemistry of Physics and Materials.

- Pilchak, A. L., Ren, F., Case, E. D., Timm, E. J, Schock, H. J., Wu, C.-I., Hogan, T. P., (October 2007). "Characterization of Dry Milled Powders of LAST (lead-antimony-silvertellurium) Thermoelectric Material,” Philosophical Magazine, v87, Issue 29, pp. 4567-4591.

- Ren, F., Case, E.D., Timm, E.J., and Schock, H.J., (2007). "Young's Modulus as a Function of Composition for an N-Type Lead-Antimony-Silver-Telluride (LAST) Thermoelectric Material,” Philosophical Magazine, v.87, Issue 31, pp. 4907-4934.

- $\quad$ Ren, F., Case, E.D., Timm, E.J., and Schock, H.J., (2007). "Hardness as a Function of Composition for N-Type Last Thermoelectric Material,” Applied Physics Letters.

- $\quad$ Ren, F., Case, E.D., Timm, E.J., Jacobs, M.D., Schock, H.J., (2007). “Weibull Analysis of the Biaxial Fracture Strength of a Cast P-Type Last-T Thermoelectric Material,” Philosophical Magazine Letters, 86 [10]: pp. 673-682.

- Zhu, B., Schock, H.J., Hogan, T., and Shih, T. I-P., (Jan. 2006) "Natural Convection and Radiation Heat Transfer in High-Temperature Thermoelectric Couples," AIAA Paper 20060574, Aerospace Sciences Meeting, Reno, Nevada.

- Harris, R., Schock, H.J., and Hogan, T., and Shih, T., (Jan. 2006) "Heat Transfer and Electric Current Flow in a Thermoelectric Couple," AIAA Paper 2006-0575, Aerospace Sciences Meeting, Reno, Nevada. 
Appendix B. Processing Conditions for Skutterudite Powder Processing

Table B1. Dry milling conditions for skutterudite powders.

\begin{tabular}{|c|c|c|c|c|c|c|}
\hline Ingot & $\begin{array}{c}\text { Batch } \\
\text { Number }\end{array}$ & Batch Size & Date & $\begin{array}{c}\text { Media } \\
\text { Diameter }\end{array}$ & $\begin{array}{c}\text { Media } \\
\text { Amount }\end{array}$ & $\begin{array}{c}\text { Media } \\
\text { Type }\end{array}$ \\
\hline \multirow[t]{4}{*}{ N-SKD-Min2 } & B1 & $30.00 \mathrm{~g}$ & 8 March 2009 & $10 \mathrm{~mm}$ & $390.77 \mathrm{~g}$ & WC \\
\hline & B2 & $25.00 \mathrm{~g}$ & 24 March 2009 & $\begin{array}{c}20 \mathrm{~mm} \\
6 \mathrm{~mm}\end{array}$ & $\begin{array}{l}226.20 \mathrm{~g} \\
170.03 \mathrm{~g}\end{array}$ & SS \\
\hline & B3 & $23.00 \mathrm{~g}$ & 26 March 2009 & $20 \mathrm{~mm}$ & $227.21 \mathrm{~g}$ & SS \\
\hline & B4 & $23.05 g$ & 1 April 2009 & $10 \mathrm{~mm}$ & $233.31 \mathrm{~g}$ & SS \\
\hline \multirow{4}{*}{$\begin{array}{c}\text { ETN-SKD- } \\
10 \\
\end{array}$} & B1 & $24.33 g$ & 2 June 2009 & $10 \mathrm{~mm}$ & $229.90 \mathrm{~g}$ & SS \\
\hline & B2 & $24.00 \mathrm{~g}$ & 4 June 2009 & $10 \mathrm{~mm}$ & $229.99 \mathrm{~g}$ & SS \\
\hline & B3 & $23.88 \mathrm{~g}$ & 5 June 2009 & $10 \mathrm{~mm}$ & $230.13 g$ & SS \\
\hline & B4 & $23.88 \mathrm{~g}$ & 8 June 2009 & $10 \mathrm{~mm}$ & $230.08 \mathrm{~g}$ & SS \\
\hline \multirow[t]{4}{*}{ JSp-SKD-15 } & B1 & $23.81 \mathrm{~g}$ & 16 June 2009 & $20 \mathrm{~mm}$ & $226.49 \mathrm{~g}$ & SS \\
\hline & B2 & N/A & N/A & N/A & N/A & N/A \\
\hline & B3 & N/A & N/A & N/A & N/A & N/A \\
\hline & B4 & N/A & N/A & N/A & N/A & N/A \\
\hline \multirow[t]{4}{*}{ JSN-SKD-14 } & B1 & N/A & N/A & N/A & $\mathrm{N} / \mathrm{A}$ & N/A \\
\hline & B2 & N/A & N/A & N/A & N/A & N/A \\
\hline & B3 & N/A & N/A & N/A & $\mathrm{N} / \mathrm{A}$ & N/A \\
\hline & B4 & N/A & N/A & N/A & N/A & N/A \\
\hline
\end{tabular}


Table B2. Wet milling conditions.

\begin{tabular}{|c|c|c|c|c|c|c|c|}
\hline Ingot & $\begin{array}{c}\text { Batch } \\
\text { Number }\end{array}$ & $\begin{array}{c}\text { Batch } \\
\text { Size }\end{array}$ & Date & $\begin{array}{c}\text { Media } \\
\text { Diameter }\end{array}$ & $\begin{array}{c}\text { Media } \\
\text { Amount }\end{array}$ & $\begin{array}{c}\text { Milling } \\
\text { Fluid }\end{array}$ & $\begin{array}{c}\text { Media } \\
\text { Type }\end{array}$ \\
\hline \multirow[t]{4}{*}{$\begin{array}{c}\text { N-SKD- } \\
\text { Min2 }\end{array}$} & B1 & $26.60 \mathrm{~g}$ & 8 March 2009 & $\begin{array}{c}20 \mathrm{~mm} \\
6 \mathrm{~mm}\end{array}$ & $\begin{array}{l}412.82 \mathrm{~g} \\
100.20 \mathrm{~g}\end{array}$ & $\begin{array}{c}25 \mathrm{~mL} \\
\text { Hexane }\end{array}$ & WC \\
\hline & B2 & $20.55 g$ & 25 March 2009 & $\begin{array}{c}20 \mathrm{~mm} \\
6 \mathrm{~mm}\end{array}$ & $\begin{array}{l}226.22 \mathrm{~g} \\
170.32 \mathrm{~g}\end{array}$ & $\begin{array}{c}25 \mathrm{~mL} \\
\text { Hexane }\end{array}$ & SS \\
\hline & B3 & $21.00 \mathrm{~g}$ & 26 March 2009 & $\begin{array}{c}20 \mathrm{~mm} \\
6 \mathrm{~mm}\end{array}$ & $\begin{array}{l}226.30 \mathrm{~g} \\
173.23 \mathrm{~g}\end{array}$ & $\begin{array}{l}25 \mathrm{~mL} \\
\text { Hexane }\end{array}$ & SS \\
\hline & B4 & $18.70 \mathrm{~g}$ & 3 April 2009 & $\begin{array}{c}20 \mathrm{~mm} \\
3 \mathrm{~mm}\end{array}$ & $\begin{array}{l}226.14 \mathrm{~g} \\
170.10 \mathrm{~g}\end{array}$ & $\begin{array}{l}25 \mathrm{~mL} \\
\text { Hexane }\end{array}$ & SS \\
\hline \multirow[t]{4}{*}{$\begin{array}{c}\text { ETN- } \\
\text { SKD-10 }\end{array}$} & B1 & $22.01 \mathrm{~g}$ & 4 June 2009 & $\begin{array}{c}20 \mathrm{~mm} \\
6 \mathrm{~mm}\end{array}$ & $\begin{array}{l}226.05 \mathrm{~g} \\
170.10 \mathrm{~g}\end{array}$ & $\begin{array}{c}25 \mathrm{~mL} \\
\text { Ethanol }\end{array}$ & SS \\
\hline & B2 & $22.00 \mathrm{~g}$ & 4 June 2009 & $\begin{array}{c}20 \mathrm{~mm} \\
6 \mathrm{~mm}\end{array}$ & $\begin{array}{l}226.13 g \\
170.14 g\end{array}$ & $\begin{array}{c}25 \mathrm{~mL} \\
\text { Ethanol }\end{array}$ & SS \\
\hline & B3 & $21.50 \mathrm{~g}$ & 5 June 2009 & $\begin{array}{c}20 \mathrm{~mm} \\
6 \mathrm{~mm}\end{array}$ & $\begin{array}{l}226.12 \mathrm{~g} \\
170.23 \mathrm{~g}\end{array}$ & $\begin{array}{c}25 \mathrm{~mL} \\
\text { Ethanol }\end{array}$ & SS \\
\hline & B4 & $22.00 \mathrm{~g}$ & 8 June 2009 & $\begin{array}{c}20 \mathrm{~mm} \\
6 \mathrm{~mm}\end{array}$ & $\begin{array}{l}226.02 \mathrm{~g} \\
170.26 \mathrm{~g}\end{array}$ & $\begin{array}{c}25 \mathrm{~mL} \\
\text { Ethanol }\end{array}$ & SS \\
\hline \multirow[t]{4}{*}{$\begin{array}{c}\text { JSp- } \\
\text { SKD-15 }\end{array}$} & B1 & $18.00 \mathrm{~g}$ & 16 June 2009 & $\begin{array}{c}20 \mathrm{~mm} \\
6 \mathrm{~mm}\end{array}$ & $\begin{array}{l}230.23 \mathrm{~g} \\
170.05 \mathrm{~g}\end{array}$ & $\begin{array}{c}25 \mathrm{~mL} \\
\text { Ethanol }\end{array}$ & SS \\
\hline & B2 & $23.80 \mathrm{~g}$ & 17 June 2009 & $\begin{array}{c}20 \mathrm{~mm} \\
6 \mathrm{~mm}\end{array}$ & $\begin{array}{l}226.42 \mathrm{~g} \\
170.39 \mathrm{~g}\end{array}$ & $\begin{array}{c}25 \mathrm{~mL} \\
\text { Ethanol }\end{array}$ & SS \\
\hline & B3 & $23.80 \mathrm{~g}$ & 18 June 2009 & $\begin{array}{c}20 \mathrm{~mm} \\
6 \mathrm{~mm}\end{array}$ & $\begin{array}{l}226.41 \mathrm{~g} \\
170.47 \mathrm{~g}\end{array}$ & $\begin{array}{c}25 \mathrm{~mL} \\
\text { Ethanol }\end{array}$ & SS \\
\hline & B4 & $23.80 \mathrm{~g}$ & 25 June 2009 & $\begin{array}{c}20 \mathrm{~mm} \\
6 \mathrm{~mm}\end{array}$ & $\begin{array}{l}226.44 \mathrm{~g} \\
170.08 \mathrm{~g}\end{array}$ & $\begin{array}{c}37 \mathrm{~mL} \\
\text { Ethanol }\end{array}$ & SS \\
\hline \multirow[t]{4}{*}{$\begin{array}{c}\text { JSN- } \\
\text { SKD-14 }\end{array}$} & B1 & $23.50 \mathrm{~g}$ & 30 July 2009 & $\begin{array}{c}20 \mathrm{~mm} \\
6 \mathrm{~mm}\end{array}$ & $\begin{array}{l}226.08 \mathrm{~g} \\
170.02 \mathrm{~g}\end{array}$ & $\begin{array}{c}25 \mathrm{~mL} \\
\text { Ethanol }\end{array}$ & SS \\
\hline & B2 & $23.50 \mathrm{~g}$ & 31 July 2009 & $\begin{array}{c}20 \mathrm{~mm} \\
6 \mathrm{~mm}\end{array}$ & $\begin{array}{l}226.15 \mathrm{~g} \\
170.32 \mathrm{~g}\end{array}$ & $\begin{array}{c}25 \mathrm{~mL} \\
\text { Ethanol }\end{array}$ & SS \\
\hline & B3 & $23.50 \mathrm{~g}$ & 2 August 2009 & $\begin{array}{c}20 \mathrm{~mm} \\
6 \mathrm{~mm}\end{array}$ & $\begin{array}{l}226.21 \mathrm{~g} \\
170.44 \mathrm{~g}\end{array}$ & $\begin{array}{c}37 \mathrm{~mL} \\
\text { Ethanol }\end{array}$ & SS \\
\hline & B4 & $23.50 \mathrm{~g}$ & 3 August 2009 & $\begin{array}{c}20 \mathrm{~mm} \\
6 \mathrm{~mm}\end{array}$ & $\begin{array}{l}226.23 g \\
170.43 g\end{array}$ & $\begin{array}{c}42 \mathrm{~mL} \\
\text { Ethanol }\end{array}$ & SS \\
\hline
\end{tabular}

